\title{
Calibration Experiments of a Laser Scanner
}

Building and Fire Research Laboratory

Gaithersburg, MD 20899

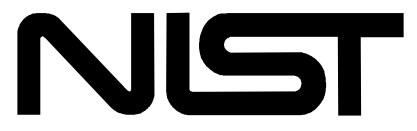

United States Department of Commerce

Technology Administration

National Institute of Standards and Technology 


\section{Calibration Experiments of a Laser Scanner}

Geraldine S. Cheok

Stefan Leigh

Andrew Rukhin

September 2002

Building and Fire Research Laboratory

National Institute of Standards and Technology

Gaithersburg, MD 20899

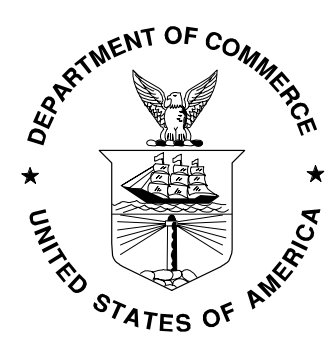

\section{U.S. Department of Commerce}

Donald L. Evans, Secretary

Technology Administration

Phillip J. Bond, Under Secretary

Technology Administration

National Institute of Standards and Technology

Arden L. Bement, Director 


\begin{abstract}
The potential applications of laser scanners or LADARs (Laser Detection and Ranging) are numerous, and they cross several sectors of the industry - construction, large-scale manufacturing, remote sensing, national defense. A LADAR is an instrument which can rapidly capture 3-D data of a scene in the form of $x, y, z$ points. The confidence in end products of the applications - 3D models, positioning, derived quantities - depends largely on the accuracy and precision of the laser scanner. For global uncertainty analysis via error propagation, specific instrumental uncertainties need to be determined.

This report chronicles exploratory experiments conducted at the National Institute of Standards and Technology to characterize a LADAR, focusing on accuracy and precision. The specific variables considered were distance, angle of incidence, and target color. Other characteristics of the LADAR such as the returned intensity and beam divergence were also examined.

Initial findings indicate no obvious global color effect on accuracy although range-specific biases were observed. Decreased measurement accuracy is observed for higher angles of incidence. Reflectivity contributes to measurement error with highly reflective targets exhibiting large errors in the shorter ranges and low reflective targets being less precise in the longer ranges.

These initial efforts are expected to contribute to the understanding of what is required to calibrate similar sensors in terms of significant parameters, test procedures, and calibration facility requirements.
\end{abstract}




\section{TABLE OF CONTENTS}

ABSTRACT .iii CONTENTS V

1.0 INTRODUCTION 1

2.0 EXPERIMENTAL PARAMETERS AND PROCEDURES ........................................ 5

2.1 Data Set 1 - Effect of Color on Range Accuracy .............................................. 6

2.2 Data Set 2 - Effect of Distance $(0 \mathrm{~m}$ to $60 \mathrm{~m})$ on Range Accuracy ....................... 7

2.3 Data Set 3 - Extension of Data Set $2(60 \mathrm{~m}$ to $108 \mathrm{~m})$....................................... 8

2.4 Data Set 4 - Effect of Distance $(5 \mathrm{~m}$ to $160 \mathrm{~m}$ ) on Range Accuracy ..................... 8

2.5 Data Set 5 - Effect of Distance (10 m to $150 \mathrm{~m}$ ) on Range Accuracy and Effect of Distance on Intensity Value ............................................................. 9

2.6 Data Set 6 - Effect of Angle Incidence on Accuracy ...................................... 12

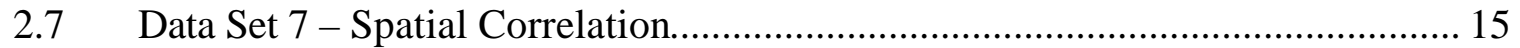

2.8 Data Set 8 - Autocorrelation..................................................................... 16

3.0 ANALYSIS AND DISCUSSION OF RESULTS ................................................ 17

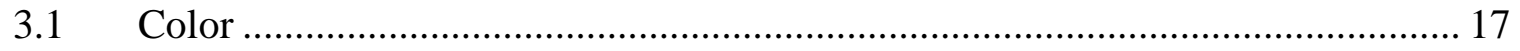

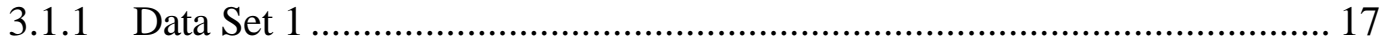

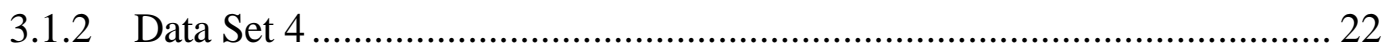

3.1.3 Data Set 5 ................................................................................... 27

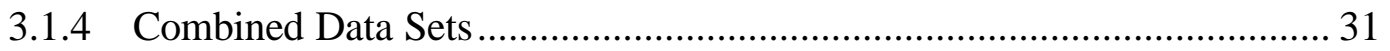

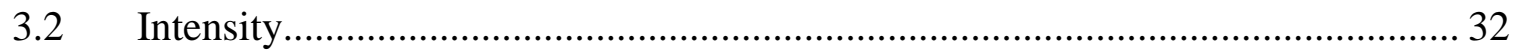

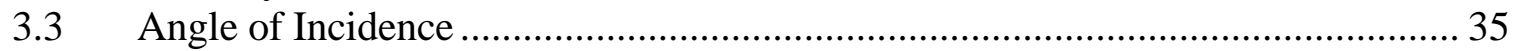

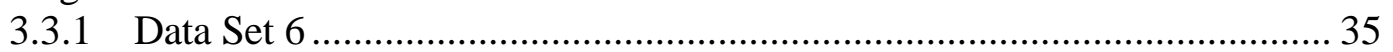

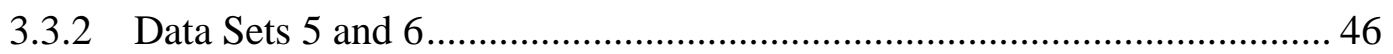

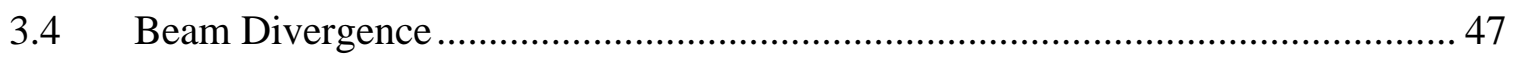

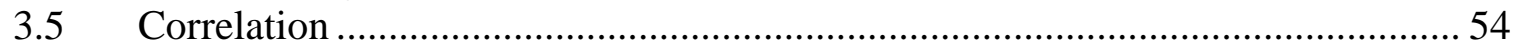

3.5.1 Temporal Autocorrelation - Data Set 8 ........................................... 55

3.5.2 Spatial Autocorrelation - Data Set 7 ........................................... 57

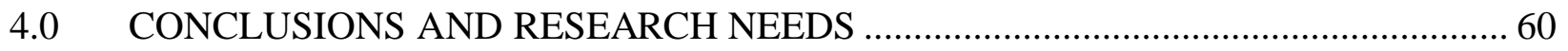

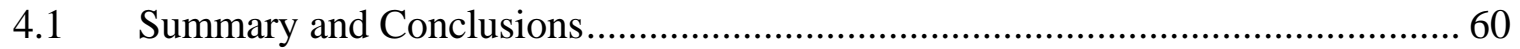

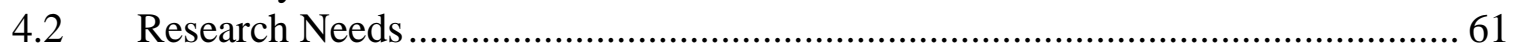

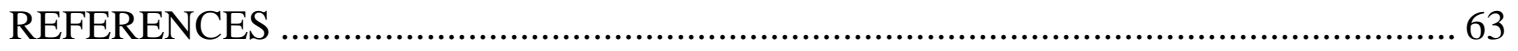

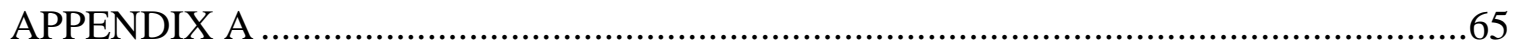

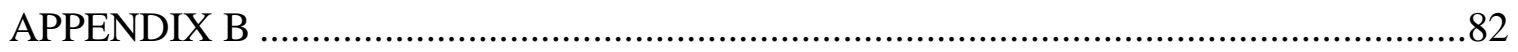




\subsection{INTRODUCTION}

LADAR (laser detection and ranging) technology has been available since the 1970s. Its full potential, however, was not fully realized until recently (1990s) because of inhibitive cost and poor reliability of the early devices. However, advances in micro-chip lasers, optics, MEMS (microelectromechanical systems) technology, and computers have led to increases in speed of data acquisition, range accuracy and reliability as well as reduction in size and costs. As the use of LADARs increases, the need is growing to characterize their performance and to develop confidence limits for the LADAR data and their end products. There has been little effort in this area thus far.

A LADAR is an instrument which can rapidly capture 3-D data of a scene in the form of $x, y, z$ points (point clouds, Fig. 1.1) as contrasted to photography, which gives a 2-D projection. In general, LADARs return two pieces of information, range (=distance) and intensity (function of the strength of the return signal). Additionally, some LADARS can obtain other spectral data such as $\mathrm{R}(\mathrm{ed}), \mathrm{G}($ reen), $\mathrm{B}$ (lue) values which can be used to aid in object identification.

This report chronicles exploratory calibration experiments conducted at the National Institute of Standards and Technology (NIST). These experiments, conducted over a period of three years, served three purposes: 1) to characterize the performance of a laser scanner with the focus on accuracy and precision calibrations of the LADAR, 2) as a learning process for the use of and familiarization with the LADAR and 3) to address needs as they arose such as the examination of intensity for bar code recognition. The experimental variables included distance, target color (reflectivity) and angle of incidence. Additionally, the effect target material and distance on intensity, and the laser beam divergence were examined. 


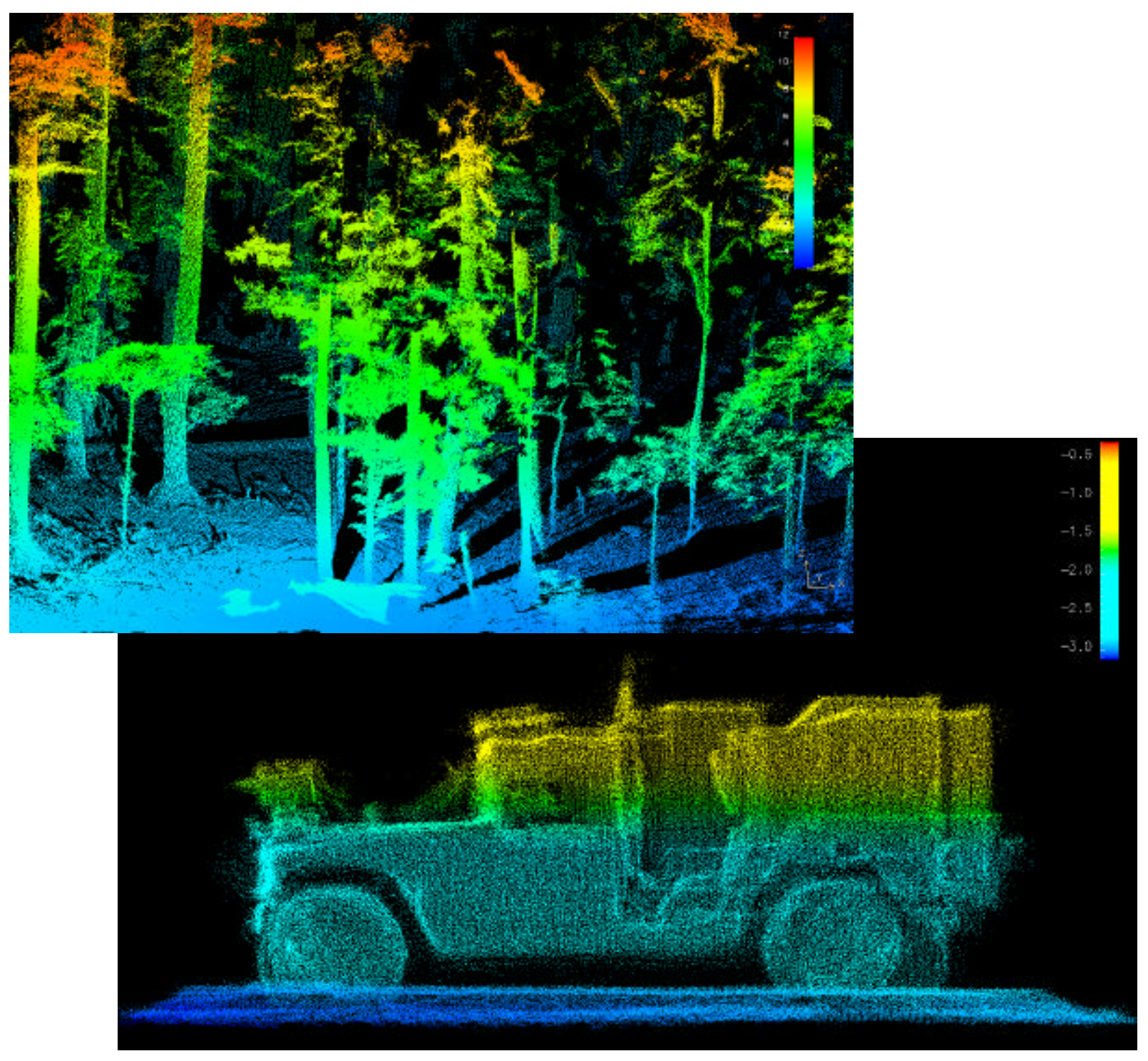

Figure 1.1. Point Clouds of a Wooded Area (top) and a Vehicle (bottom).

The potential applications of LADARs are remendous, and they cross several sectors of the industry - construction, large-scale manufacturing, remote sensing, national defense (military). Some applications for LADAR data are shown in Fig. 1.2. These applications include creating 3-D models for as-builts, reverse engineering, surveying, urban planning, terrain mapping, and machine automation. 


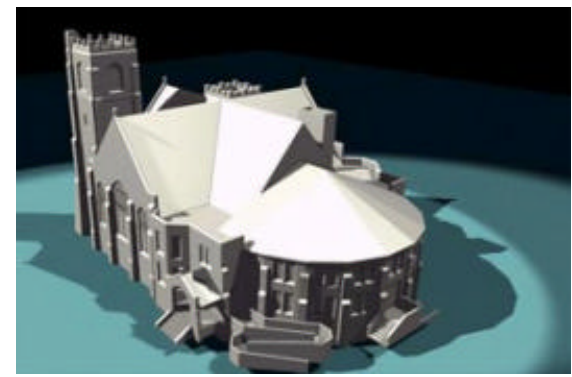

Courtesy of Quantapoint
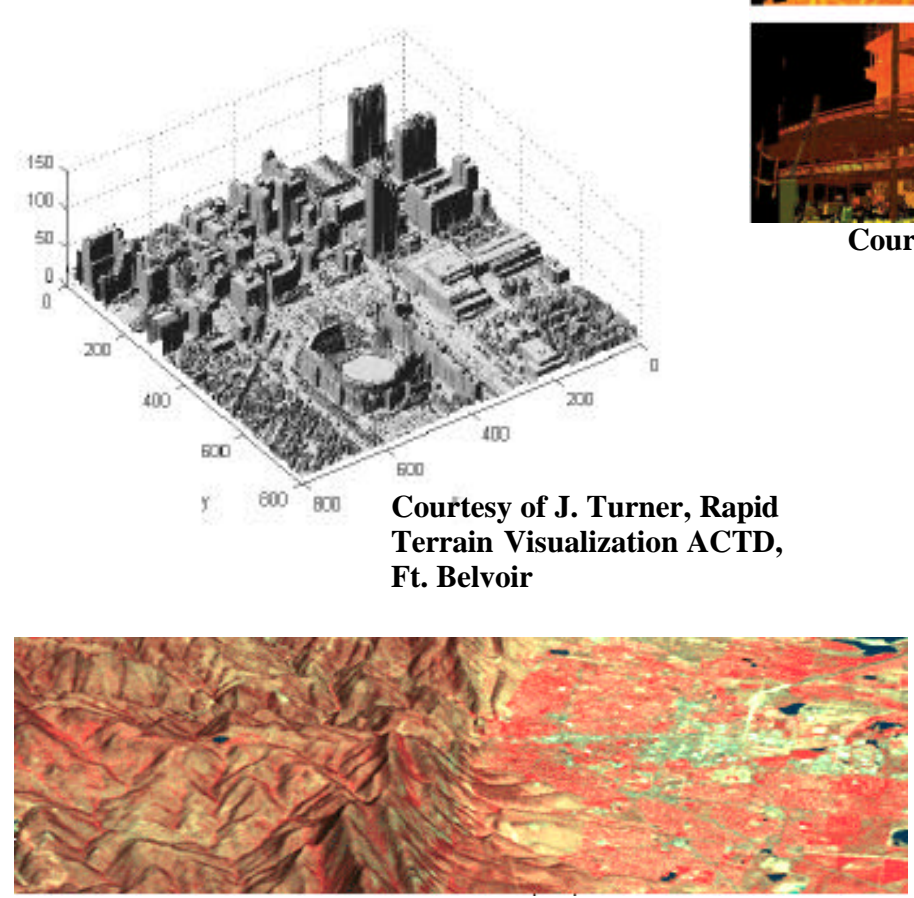

Courtesy of Computer Terrain Mapping Inc.

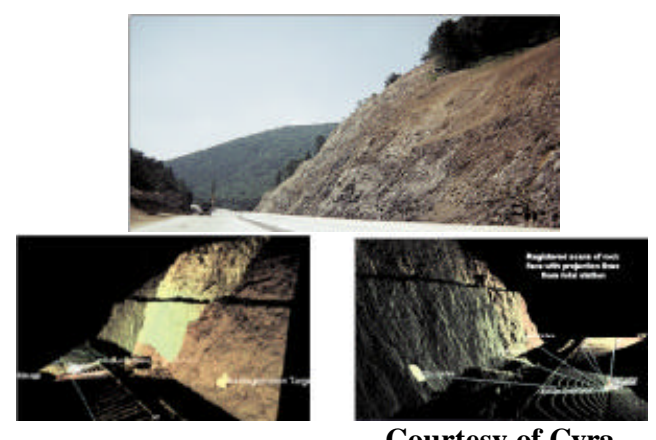

Courtesy of Cyra

Figure 1.2. Some Applications for LADAR Data -3D Models, Terrain Mapping, Surveying, Quality Control*.

The confidence in final products from the measurements - 3D models, positioning, derived quantities (e.g., volume), etc. - depends largely on the accuracy and precision of the laser scanner. Accuracy is defined by how close a measurement is to the true value, while precision is

\footnotetext{
* Certain trade names and company products are mentioned in the text or identified in an illustration in order to adequately specify the experimental procedure and equipment used. In no case does such an identification imply recommendation or endorsement by the National Institute of Standards and Technology, nor does it imply that the products are necessarily the best available for the purpose.
} 
defined as the scatter or variation in repeated measurements. Generic instrumental uncertainties propagate through to end results.

A first, basic approach to the estimation of uncertainties associated with reconstruction of scene features is to incorporate propagation-of-error ("delta method") based estimates of error [Taylor, 1997] to the surface generation (meshing) algorithms. Roughly, this requires first order estimates of error at any point in the mesh, i.e., standard error (or variance) of distance measurements, and if possible second order estimates of spatial covariances between points. Spatial covariance of measurements taken in close proximity leads to refinement $\left(2^{\text {nd }}\right.$ order terms) in propagation-of-error generated standard errors. Negative covariances may be exploited to improve the error estimates. Positive covariances inflate error estimates. The standard temporal correlation measure is the serial autocorrelation. Presence of significant autocorrelation indicates that standard error propagation methods may produce results that are better than warranted. This is the underlying motivation for all the issues considered in this publication. We examine factors influencing the accuracy and precision of LADAR estimated distances (first order), and consider temporal autocorrelation and spatial covariance (second order) briefly as well in this report.

Besides instrument error, the computational techniques used to process the data contribute to the accuracy of estimating such derived quantities as volume and object position/dimensions. For example, model accuracy is affected by the number of points chosen to create the model (full or subset), and for a subset of points, selection of the points and number of points selected; if the point cloud consists of two of more scans obtained from different locations, how well were the different scans registered to a common reference frame; and how was noise filtered out.

This report deals primarily with the accuracy and precision (repeatability) of the hardware, i.e., the scanner, and not the software, and it describes experiments and statistical analyses conducted in order to determine the effect of color and angle of incidence on the accuracy and precision of the scanner's range measurements. As a result of additional information gathered during the conduct of the range calibrations, the effect of distance on the signal intensity and laser divergence could also be studied. These initial efforts contribute to the understanding of what is required to calibrate similar sensors in terms of significant parameters, test procedures, and calibration facility requirements. Other similar efforts may be found in Marshall and Gilby [2001], Marshall et. al. [2001], Collier [1998], El-Hakim et. al. [1995], and Kweon et. al. [1991].

This report is organized as follows: An introduction is given in Chapter 1. Chapter 2 describes the different data sets and the conditions under which the data were collected. A discussion of the experimental results and an analysis of the results are given in Chapter 3. In Chapter 4, the findings based on the results of the experiments and research needs are presented. 


\subsection{EXPERIMENTAL PARAMETERS AND PROCEDURES}

The laser scanner records range and intensity data. The maximum range of the tested laser scanner is $150 \mathrm{~m}$, and the manufacturer's specified accuracy varies from $\pm 2 \mathrm{~cm}$ (best case) to $\pm 5 \mathrm{~cm}$ (worst case). The range is based on the time-of-flight of the returned signal with the triggering of the detector based on the strength of return signal. The intensity data are integer values ranging from 0 (lowest) to 225 (highest) and are also a function of the strength of the return signal.

The signal strength is primarily dependent on the distance and reflectivity of the object. The reflectivity of the object is a function of the object's color, texture (smooth, rough), specularity. Since the scanner would be used to scan scenes that contain objects of varying colors and textures, a pilot experiment to assess the influence of color and/or texture on the accuracy of the range measurements was conducted. As more understanding and hands-on experience with the scanner was gained, the effect of angle of incidence emerged as a significant effect on the range accuracy and precision. Thus, additional tests were conducted to determine the effect of angle of incidence on the range accuracy and precision. Also, as discussed in Section 2.5, data on the intensity value were collected as the need to determine the effect of distance on the intensity value arose. All experiments took place at the NIST, Gaithersburg campus at four indoor sites.

All experimental range data were collected in the form of $(x, y)$ pairs where $y=$ LADAR distance measurement and $x=$ independent measurement of the distance, e.g., total station, interferometer or tape measure. This enables assessment of the accuracy of the LADAR as well as its precision.

A summary of the varying test conditions used to study the effect of distance, color, and angle of incidence on the range accuracy is given in Table 2.1 (an " $\mathrm{X}$ " in the column indicates that the parameter was studied). Color, specularity, and texture were varied between representative extremes and combined in fractional factorial fashion to examine the potential effects of each.

Table 2.1. Test Parameters.

\begin{tabular}{|c|c|c|c|}
\hline Material & Distance & $\begin{array}{l}\text { Angle of } \\
\text { Incidence }\end{array}$ & Intensity \\
\hline White, smooth, shiny & $\mathrm{X}$ & $\mathrm{X}$ & $\mathrm{X}$ \\
\hline White, smooth, not shiny & $\mathrm{X}$ & & \\
\hline Yellow, smooth, not shiny & $\mathrm{X}$ & & \\
\hline Pink, smooth, not shiny & $\mathrm{X}$ & & \\
\hline Green, smooth, not shiny & $\mathrm{X}$ & & \\
\hline Dark Gray, rough, not shiny & $\mathrm{X}$ & & \\
\hline Black, smooth, shiny & $\mathrm{X}$ & $\mathrm{X}$ & $\mathrm{X}$ \\
\hline Black, smooth, not shiny & $\mathrm{X}$ & & \\
\hline Silver (uncoated aluminum) & $X$ & $\mathrm{X}$ & $\mathrm{X}$ \\
\hline $\begin{array}{l}\text { 3M Long Distance Performance } \\
\text { (LDP) - Reflective material }\end{array}$ & $\mathrm{X}$ & & $\mathrm{X}$ \\
\hline
\end{tabular}


The most desirable distance calibration is an absolute distance calibration where the distance from the scanner to the target is accurately determined by independent means. However, for the first distance calibration, Data Set 1, the laser distances were compared with distances measured with an interferometer. The accuracy of the interferometer is several orders of magnitude better than that of the laser scanner. The interferometer is, however, a relative distance instrument. Therefore, the first calibration is a relative distance calibration. The subsequent data sets, Data Sets 2 to 8, are absolute distance calibrations.

No facility currently available at NIST allows for long distance ( $\geq 100 \mathrm{~m})$ calibration. Therefore, the absolute distance calibrations were conducted at the following four locations: 1.) Metrology Building, Bldg. 220 (60 m maximum clear line of sight) 2.) Building Research, Bldg. 226 ( $\approx 110 \mathrm{~m}$ maximum clear line of sight) 3.) Corridor spanning several buildings ( $>150$ $\mathrm{m}$ maximum clear line of sight) 4.) Tunnel, Bldg. 101 ( $>150 \mathrm{~m}$ maximum clear line of sight). Except for the first location (Bldg. 220), environmental control was not possible at any of these sites. An ideal site would provide the following requirements: 1.) environmentally controlled (temperature, humidity, negative and positive barometric pressure control, uniform diffuse lighting) with multiple sensors throughout the lab to monitor the environmental conditions 2.) open space that is about $10 \mathrm{~m}$ (width) by $150 \mathrm{~m}$ to $200 \mathrm{~m}$ (length) 3.) controlled access into lab for eye safety and for ease of calibration (i.e., view of target not obscured by personnel walking between the target the scanner), 4) level concrete floor for installation of permanent benchmarks, and 5) diffuse black walls and floor.

\subsection{Data Set 1 - Effect of Color on Range Accuracy}

This set of measurements was conducted in an enclosed temperature controlled room located in the Metrology Building, Building 220 with fluorescent lighting where the temperature was held at approximately $20^{\circ} \mathrm{C}$. The maximum range achievable in this room is about $60 \mathrm{~m}$. A heterodyne interferometer was used as the basis of comparison for the laser measurements. The accuracy of the interferometer is $(0.93 \mu \mathrm{m} / \mathrm{m}) /{ }^{\circ} \mathrm{C}$ and $(0.36 \mu \mathrm{m} / \mathrm{m}) / \mathrm{mm}$ of $\mathrm{Hg}$ (reference to $20{ }^{\circ} \mathrm{C}$ and $1013 \mathrm{hPa}$ ). The environmental conditions for the experiments in Data Set 1 are given in Table 2.2.

Table 2.2. Environmental Conditions for Data Set 1.

\begin{tabular}{|c|c|c|c|c|c|c|}
\hline \multirow{2}{*}{ Test Date } & \multicolumn{2}{|c|}{$\begin{array}{c}\text { Temperature } \\
\left({ }^{\circ} \mathbf{C}\right)\end{array}$} & \multicolumn{2}{c|}{$\begin{array}{c}\text { Relative Humidity } \\
(\mathbf{\%})\end{array}$} & \multicolumn{2}{c|}{$\begin{array}{c}\text { Barometric Pressure } \\
(\mathbf{h P a})\end{array}$} \\
\cline { 2 - 7 } & Start & End & Start & End & Start & End \\
\hline July 14, 1999 & 20.8 & 20.8 & 45.5 & 45.0 & 1005.7 & 1004.6 \\
\hline July 15, 1999 & 20.1 & 20.1 & 49.3 & 49.2 & 1007.3 & 1006.6 \\
\hline Aug. 2, 1999 & 20.0 & 20.4 & 49.4 & 49.5 & 999.3 & 999.3 \\
\hline
\end{tabular}


The interferometer was set up at one end of the test bench and the laser scanner was set up slightly behind it. The target was mounted on top of a small trolley that travels the length of the test bench along a guided rail. Comparisons using the interferometer were relative distance comparisons. For example, the target was set at some arbitrary distance, usually $2 \mathrm{~m}$ to $3 \mathrm{~m}$, in front of the interferometer and a zero reading was obtained to this target. Distances thereafter were relative to this initial starting point. In the case of the laser scanner, the measurement obtained to the target set at the start point represented the distance from the target to the scanner and was used as the zero offset for measurements taken thereafter.

The procedure involved both setting up the target and leveling the trolley. Readings were then obtained at the initial position from both the interferometer and the scanner. The target was moved $2 \mathrm{~m}, 4 \mathrm{~m}, 6 \mathrm{~m}, 8 \mathrm{~m}, 10 \mathrm{~m}, 15 \mathrm{~m}, 20 \mathrm{~m}, 30 \mathrm{~m}, 40 \mathrm{~m}$, and $50 \mathrm{~m}$ beyond the start point. At each point, including the initial point, the distance was measured 25 times.

The target was made by mounting a piece of letter size [216 mm x $279 \mathrm{~mm}$ (8.5 in x 11 in)], colored paper to a piece of cardboard. The colors were white, green, yellow, pink, gray, and black. All the targets were smooth except for the gray target. The gray rough target was a sheet of 80 grit sand paper. To make the target shiny, the target was placed inside a plastic, protective cover. The data for these tests are given in Table A.1.

\subsection{Data Set 2 - Effect of Distance ( 0 m to $60 \mathrm{~m}$ ) on Range Accuracy}

The same temperature-controlled location used for collecting Data Set 1 was also used for the first series of absolute distance measurements. Several collinear points (benchmarks), marked by ball bearings, are permanently set in the floor at specified spacings. The maximum distance between the two farthest points is $60.96 \mathrm{~m}$.

The calibration procedure involved centering the scanner above one of the benchmarks and centering the target over another benchmark using a tribrach with an optical plummet. Twentyfive measurements were taken at each point. The scanner was set up at the "zero" mark and the target (white, not shiny) set up $30.48 \mathrm{~m}$ and $60.96 \mathrm{~m}$ from the scanner. The data from these tests are given in Table B.1. The environmental conditions were not recorded.

To determine the measurement error due to environmental factors, a short parametric study was conducted. The corrections for a distance of $100 \mathrm{~m}$ were computed for the following combinations of environmental conditions: temperature of $20^{\circ} \mathrm{C}$ and $30{ }^{\circ} \mathrm{C}$, barometric pressure of $1000 \mathrm{hPa}$ and $1015 \mathrm{hPa}$, relative humidity of $20 \%$ and $50 \%$, and $\mathrm{CO}_{2}$ concentration of $0.027 \%$ and $0.033 \%(0.03 \% \pm 10 \%)$. The maximum correction for $100 \mathrm{~m}$ was $+1.5 \mathrm{~mm}$ (generally occurred for cases when the temperature was $30^{\circ} \mathrm{C}$ ). Assuming the environmental conditions were similar to those for Data Set $1\left(20^{\circ} \mathrm{C}, 50 \% \mathrm{RH}, 1000 \mathrm{hPa}, 0.03 \% \mathrm{CO}_{2}\right)$, measurement error from environmental factors was $+0.6 \mathrm{~mm}$ for $100 \mathrm{~m}$. Thus, in this report, measurement errors from environmental factors are considered negligible compared to instrument error. 


\subsection{Data Set 3 - Extension of Data Set $2(60 \mathrm{~m}$ to $108 \mathrm{~m})$}

As the maximum calibration distance was $60.96 \mathrm{~m}(200 \mathrm{ft})$ for Data Set 2, two other locations were found that allowed for longer distance measurements. One location was the attic of the Building Research building, Bldg. 226. However, this location was neither environmentally controlled nor air-conditioned. The maximum distance at this location was about $108 \mathrm{~m}$.

In the attic location, the distances between markers were measured with a tape measure. The distance ranged from $60 \mathrm{~m}$ to $108 \mathrm{~m}$ (measurements were taken to augment the data obtained in the environmentally controlled lab, Data Set 2; hence no measurements were taken at the shorter distances). Only one color target was used, white not shiny, and 25 measurements were taken at each distance. The target size was $216 \mathrm{~mm} \times 279 \mathrm{~mm}(8.5 \mathrm{in} \times 11 \mathrm{in})$. The distance measurements were obtained on two different days, Jul. 28, 1999 and Aug. 2, 1999 . The recorded temperature in the attic for the runs on Jul. 28, 1999 was $27.8^{\circ} \mathrm{C}$. No temperature was recorded for the tests on Aug. 2, 1999; however, based on data from the National Weather Service, the daily temperatures for Jul. 28, 1999 and Aug. 2, 1999 were comparable. The data from these tests are given in Table B.2.

The procedure used to center the scanner and target over a point on the floor is the same. It involves setting up a surveying tripod over the point, leveling a tribrach that is attached to the tripod, centering the tribrach over the point on the floor using the optical plummet, installing the scanner/target in a tribrach and locking it in, and re-leveling and re-centering the tribrach as needed.

\subsection{Data Set 4 - Effect of Distance (5 m to $160 \mathrm{~m}$ ) on Range Accuracy}

Since the calibration distance for Data Set 3 was limited to $108 \mathrm{~m}$, another location had to be found to determine the range accuracy at $150 \mathrm{~m}$, the scanner's maximum range. The location selected was a hallway that spanned several buildings and offered a clear distance of at least $150 \mathrm{~m}$. One side of the hallway that connected the buildings consisted of large glass windows, causing temperature variations along its length. However, all the buildings and hallways were air-conditioned and the temperature should be closer on average to the temperature of $20^{\circ} \mathrm{C}$ than in the attic location (see Section 2.2 for discussion of measurement errors due to environmental factors).

The calibration distances in the hallway ranged from $5 \mathrm{~m}$ to $161 \mathrm{~m}$. Markers were laid on the floor and the distances from the markers to a reference zero position were measured using a total station. The overall accuracy of the total station was $\pm 3 \mathrm{~mm}$. The targets were white not shiny, green not shiny and black not shiny and the size of the targets was $216 \mathrm{~mm}$ x $279 \mathrm{~mm}(8.5 \mathrm{in} \times$ $11 \mathrm{in})$. To increase eye safety indoors, a filter was provided to reduce the energy of the laser. To determine the effect of the filter on the range accuracy, a series of measurements were made using the black not shiny and green not shiny targets. One set of measurements was obtained with the filter attached and another without the filter. In the previous calibrations, 25 measurements were obtained at each distance; however, it was decided that 10 measurements were sufficient to assess repeatability. Therefore, for this and subsequent data sets, 10 
measurements were obtained at each distance. Data for these experiments are given in Table B.3.

\subsection{Data Set 5 - Effect of Distance $(10 \mathrm{~m}$ to $150 \mathrm{~m})$ on Range Accuracy and Effect of Distance on Intensity Value}

The calibrations described above (Data Sets 1 to 4) were aimed at getting a rough estimate of the general accuracy of the scanner and determining if the range accuracy and precision were dependent on material color. A further calibration was to characterize the scanner's range accuracy and to determine if there were any patterns in the intensity. Rather than using the location used for Data Set 4, an alternate location was found where the lighting and temperature would be more uniform throughout the length of hallway. This location was in a "tunnel" (long underground hallway) of Bldg. 101 of NIST. There are no windows along the tunnel. The calibration distances ranged from $10 \mathrm{~m}$ to $150 \mathrm{~m}$. The various distances from the zero or reference point were obtained using a total station with an accuracy of $\pm 3 \mathrm{~mm}$.

Based on a preliminary review of Data Sets 1 to 4 , it was decided that only 3 colors would be used - white, black, and shiny silver (unpainted aluminum sheet). The target size was $406 \mathrm{~mm}$ (16 in) wide by $508 \mathrm{~mm}$ (20 in) long. The target size was chosen so that it would be larger than the size of the laser beam at $150 \mathrm{~m}$.

NIST is currently examining the utility of using LADAR intensity data for "reading" bar codes or tags for the purpose of object identification. In order to "read" the bar code, its existence has to be first established. To do this, the bar code has to have a unique feature or characteristic so that it is easily identifiable. Therefore, the bar code would have to be made of a material that makes it easily distinguishable from any background material based on the returned intensity value. It was felt that a good candidate material would be one that had a return intensity that was much higher than any other material commonly found at a construction site and that was consistently high for distances of $0 \mathrm{~m}$ to $150 \mathrm{~m}$, i.e., intensity did not drop off with distance. These requirements are essential as the returned intensity is dependent on several factors reflectance of object, distance to object, reflectance of the surrounding objects, lighting (e.g., sunlight, shade), etc. This dependency means that the intensity of a black object at $10 \mathrm{~m}$ could be the same as the intensity of a shaded white object at $50 \mathrm{~m}$ and there would be no way to determine if the object was black or white based solely on the intensity value.

As a result, a fourth target made of 3M Long Distance Performance (LDP) material was made. The 3M LDP material is a reflective prismatic lens sheeting that is used for traffic signage. This material was chosen as it was readily available, durable, and would reflect light even if angled away from the light source. A photo of the LDP material and several magnified images are shown in Figs. 2.1 and 2.2 and it can be seen that the sheeting is made up of many small prisms. 


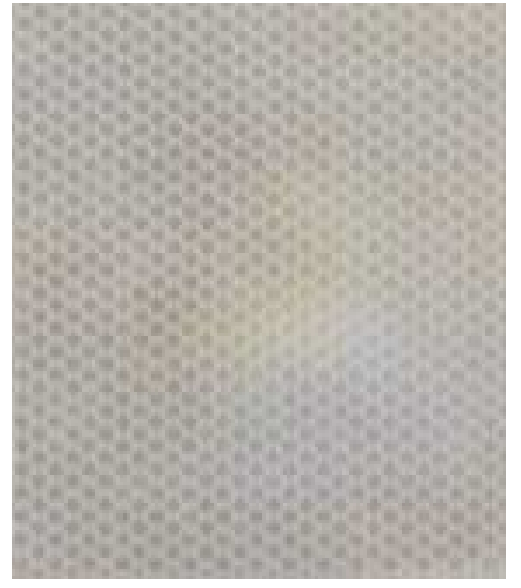

Figure 2.1. Photograph of 3M LDP

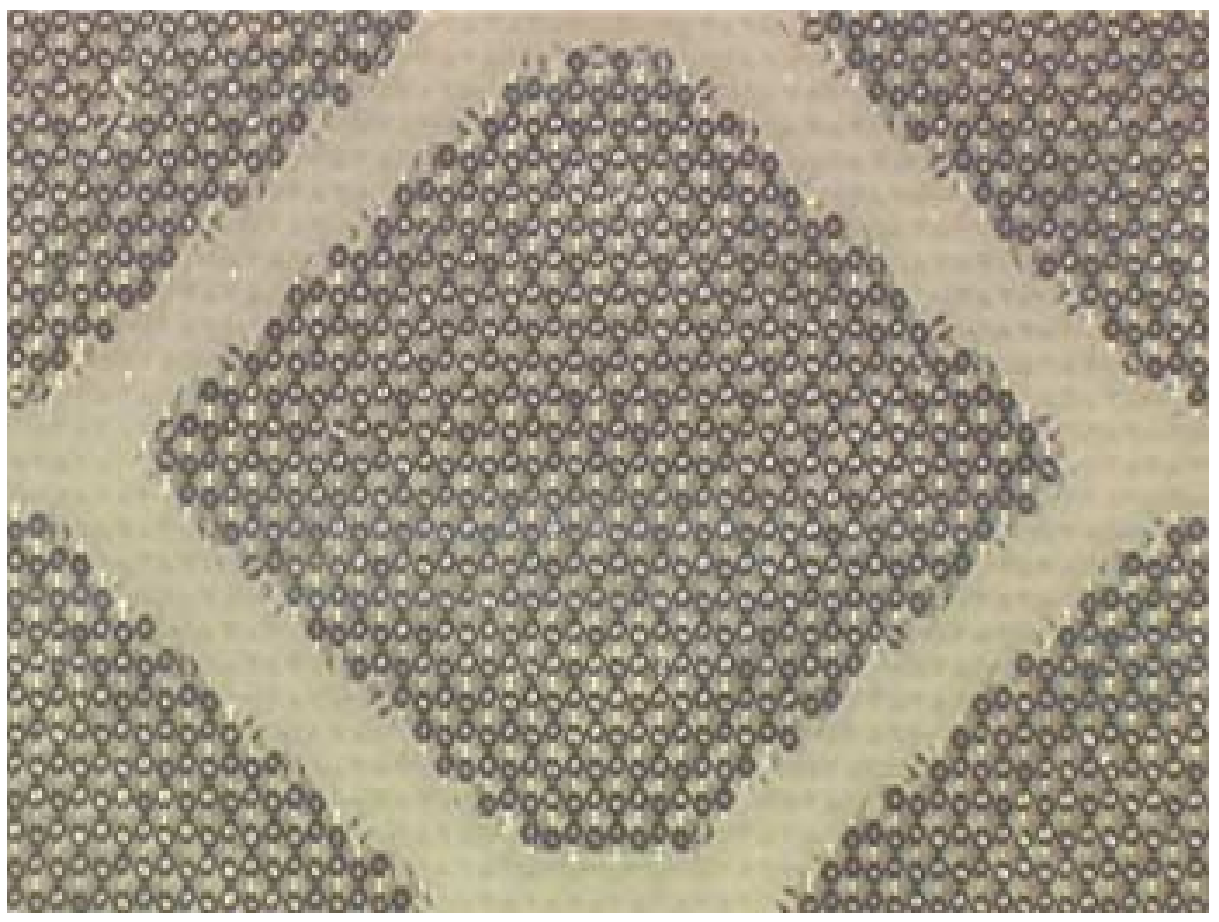

Figure 2.2a. Magnified Photo of LDP Material: Width represents $6 \mathrm{~mm}$ of surface. 


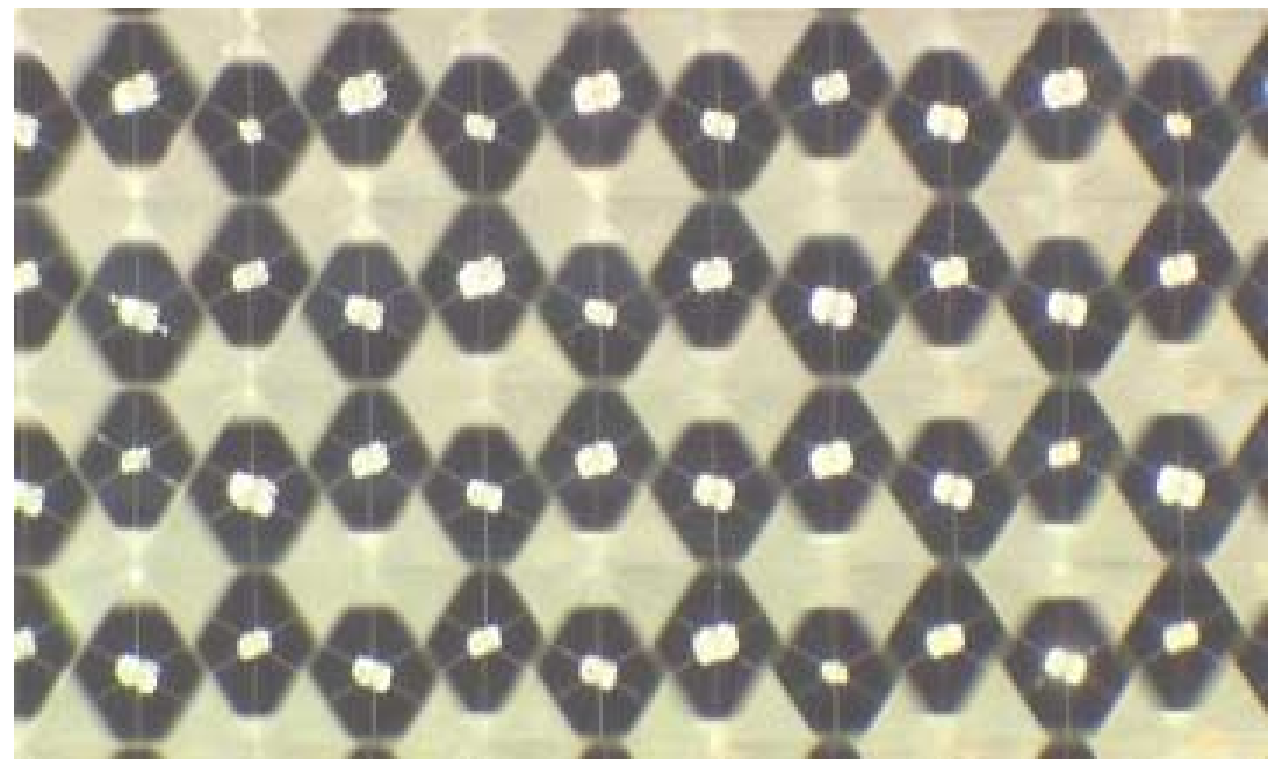

Figure 2.2b. Magnified Photo of LDP Material: Width represents $1.5 \mathrm{~mm}$ of surface.

In anticipation of the need to read bar codes angled away from the scanner, the LDP target was rotated to three positions $-0^{\circ}, 45^{\circ}$, and $60^{\circ}$ (Fig. 2.3).

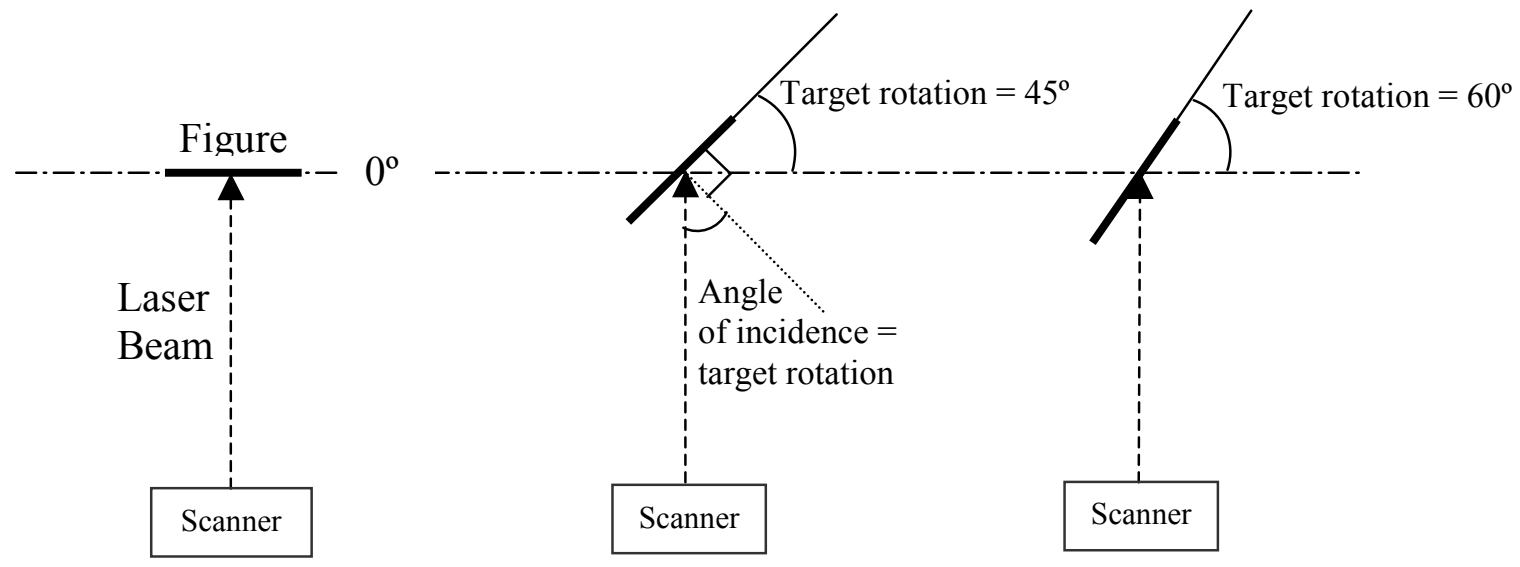

Top View of Set-up

Figure 2.3. Rotation Orientation of LDP Target.

For Data Set 5, ten measurements (both range and intensity) were recorded at each distance. The data are given in Table B.4. 


\subsection{Data Set 6 - Effect of Angle Incidence on Accuracy}

When conducting some simple scans of a box to test some post-processing software, the effect of split signals was noticed. Using the box as an example, split signals occurred when part of the signal hit the top of the box and the other part hit the floor beyond the box. The resulting instrument reported range is an interpolation between the two ranges. This creates "phantom points" (Fig. 2.4) - points that do not actually exist.

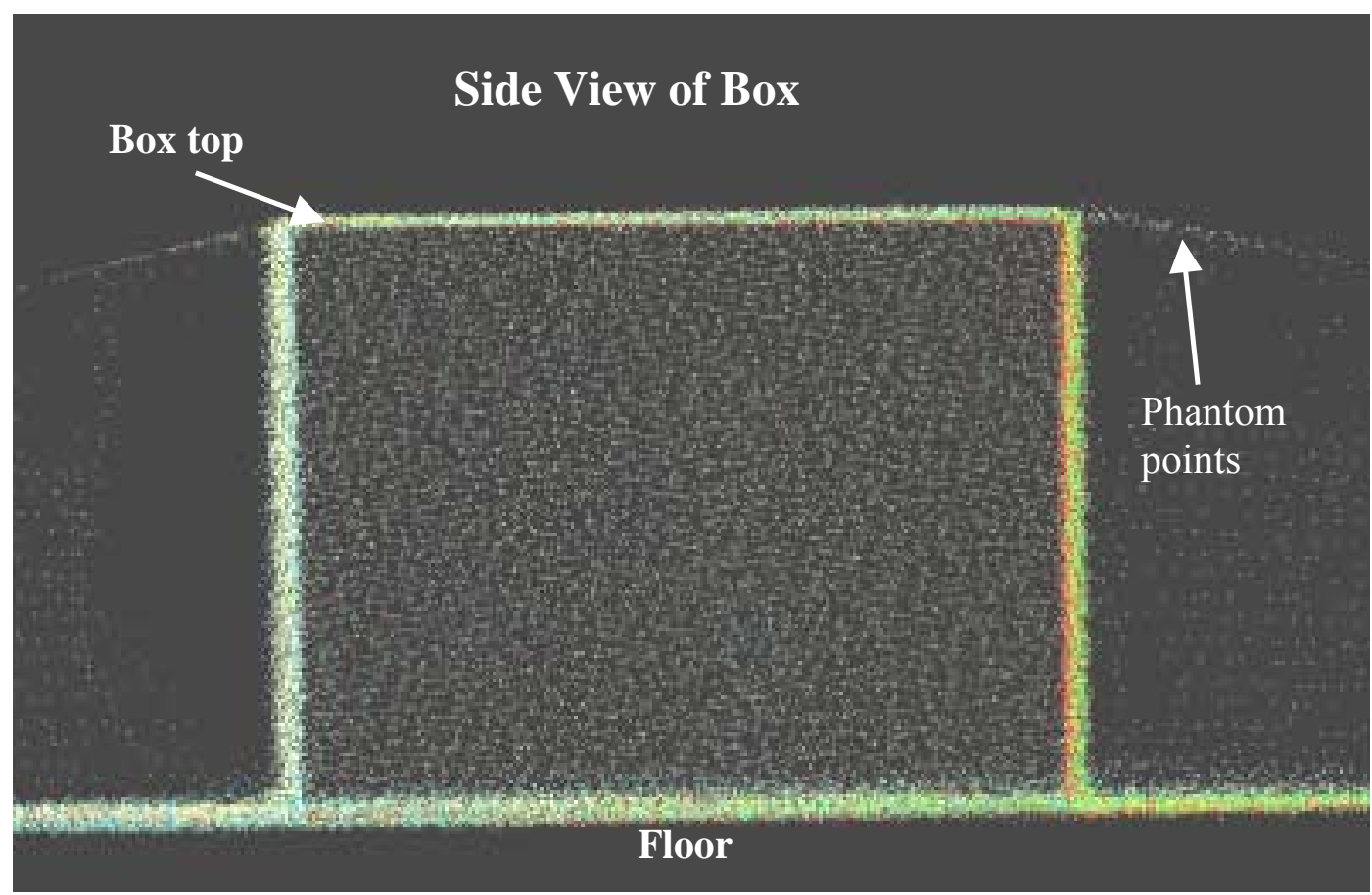

Figure 2.4. Point Cloud of Box Showing Phantom Points.

As stated by the manufacturer, the size of the laser beam as it exits the scanner is $42 \mathrm{~mm}$ (high) by $25 \mathrm{~mm}$ (wide). The beam has a divergence of $3 \mathrm{mrad}$, horizontal and vertical. This translates into a beam size of $342 \mathrm{~mm}$ (high) x $325 \mathrm{~mm}$ (wide) at $100 \mathrm{~m}$. As a result of this large beam size and the splitting of the beam, it is probable that the range accuracy will be affected by the angle of incidence (see Fig. 2.3) - the greater the angle and the longer the distance, the more adverse the effect.

To determine the effect of the angle of incidence on range accuracy, the same three targets used for Data Set 5 were reused - white, black and shiny silver (uncoated aluminum). For these tests, the targets were rotated from $0^{\circ}$ to $90^{\circ}$ in increments of $10^{\circ}$ (measurements were also obtained at $85^{\circ}$ ) with 10 measurements taken at each angle of incidence. The distance ranged from $10 \mathrm{~m}$ to 
$100 \mathrm{~m}$ in increments of $10 \mathrm{~m}$. The location of the tests was the attic of Bldg. 226. The distances from the zero reference point were measured with a tape measure*.

The conduct of these tests was more difficult than those of the previous tests. Since the tests involved rotating the target, it was important that the center of the laser beam coincide with the center of the target. This presented some difficulty as the scanner utilized an infrared (IR) laser $(\lambda=903 \mathrm{~nm})$ and the laser was pulsed with a duration of $17 \mathrm{~ns}$. As the main purpose of the scanner is to acquire data of a scene, the scanner could not be set up to automatically take the same point measurement repeatedly.

To find the center of the laser beam, an IR viewer was used to see the projection of the laser on the target. In order to see the laser projection through the IR viewer, the vicinity of the target had to be semi-dark for shorter distances (approximately $20 \mathrm{~m}$ or less) and be in total darkness for longer distances, because of the low laser power output. The procedure used to co-locate the beam center with the target center is as follows:

1. Set-up target over desired distance and attach a sheet of paper to the target.

2. Find the center of the target and mark it on the paper. Make a mark that is $79 \mathrm{~mm}$ (3.125 in) above the center mark (see Fig. 2.5).

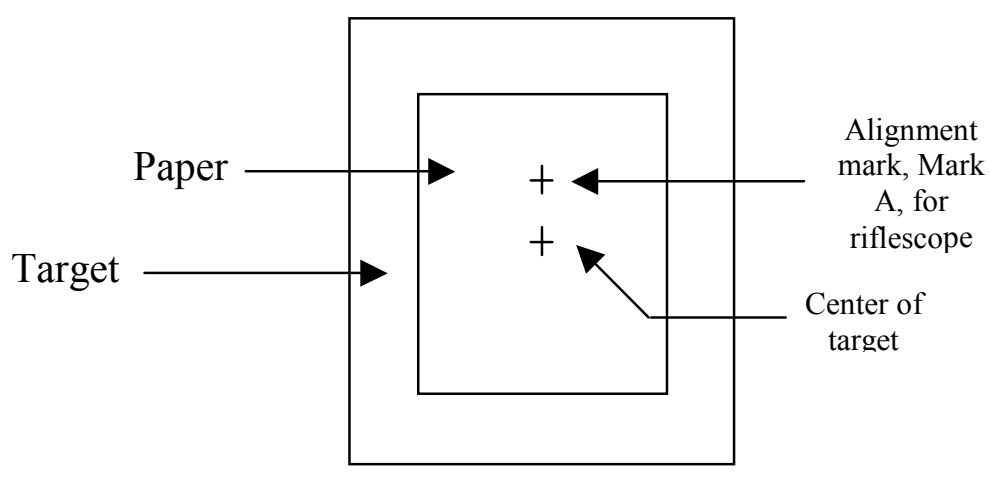

Figure 2.5. Alignment Markings on Target.

3. Align the crosshairs of the laser scanner riflescope with mark A (Fig. 2.5) on the target.

4. Turn off lights.

5. Have one person at the target with an IR viewer and another at the scanner control.

6. Have the person at the target outline the beam with a marker as the person at the control fires the laser in rapid succession.

7. Repeat Step 6 with a different person outlining the beam.

8. Turn lights on.

9. Find the center of the beam - visually.

\footnotetext{
${ }^{*}$ As the objective of this experiment was to determine the effect of angle of incidence and not to determine range accuracy, the reference distances were measured with a tape measure rather than with a total station.
} 
10. If center of the beam does not coincide with center of the target, make the necessary rotations to the scanner to align the two centers and repeat Steps 4 to 10. If it does, continue with calibration.

Some notes about the above alignment process:

1. Step 6 - outlining the beam

a. This step is very subjective, which is the main reason why a second person repeated the procedure (Step 7).

b. The difficulty in outlining the beam was increased by the need for semi- to total darkness. It was very difficult to see where the pen was on the paper to assure that what was being outlined was indeed what was seen. For future tests, it may be easier if some type of fluorescent tape were attached near the tip of the marker so that it is visible in the dark.

c. Triggering of laser - for safety reasons, the laser only fires when the scanner is in motion, i.e., when it is scanning. For the calibration, the scanner was set up to acquire only one data point. The inability of the laser to fire continuously so that the projection of the beam could be seen more clearly increased the difficulty in outlining the beam.

Another issue that should be considered is the uncertainty of the pointing accuracy of the scanner. When the scanner obtains a point scan, it moves off the point to the next point. It therefore has to move back to the previous point to take another scan of the "same" point. This issue was not considered at this stage as 1) determining the pointing accuracy of the scanner would be an entire project in itself and 2) the contribution to the total error from this issue is insignificant compared to the operator error in outlining the beam.

d. The second person was not biased by the results of the first person as the markings on the paper were not visible, due to the total darkness, when the second person was outlining the beam.

2. In all cases except for one, visual inspection of the target showed that the centers of the beam and target were aligned after the first attempt and there was no need for further adjustments to reposition the scanner; the minimum movement of the scanner is limited by the minimum resolution of the stepper motor, i.e, the minimum angle between scan points. In the one instance where the centers did not coincide, only small adjustments were necessary to align the centers of the beam and the target. This indicates that the riflescope was aligned with the axis of the laser beam and that the alignment performed in Step 3 would yield a good alignment of the target with the scanner.

The data collection for this set was accomplished over a period of 3 nonconsecutive days. The environmental conditions when the data were collected are given in Table 2.3. On Feb. 26 and 
27, 2002, the objective of the tests was to gather additional information on beam size as a function of distance and not to gather additional information on distance accuracy as a function of angle of incidence.

Table 2.3. Environmental Conditions for Data Set 6.

\begin{tabular}{|l|c|c|c|c|c|c|c|}
\hline \multicolumn{1}{|c|}{ Date } & $\begin{array}{c}\text { Distance } \\
(\mathbf{m})\end{array}$ & \multicolumn{2}{|c|}{$\begin{array}{c}\text { Temperature } \\
\left({ }^{\mathbf{0}} \mathbf{C}\right)\end{array}$} & \multicolumn{2}{c|}{$\begin{array}{c}\text { Relative Humidity } \\
(\boldsymbol{\%})\end{array}$} & \multicolumn{2}{c|}{$\begin{array}{c}\text { Barometric Pressure } \\
(\mathbf{h P a})\end{array}$} \\
\cline { 2 - 8 } & & Start & End & Start & End & Start & End \\
\hline Nov. 1, 2001 & 10 & 25.1 & 25.7 & 33.2 & 33.5 & 1007.0 & 1007.0 \\
\hline Nov. 5, 2001 & 20 & 23.8 & 23.8 & 26.5 & 26.5 & 1001.5 & 1002.0 \\
\hline Nov. 5, 2001 & 30 & 24.0 & 23.8 & 27.0 & 26.5 & 1001.5 & 1001.5 \\
\hline Nov. 28, 2001 & 40 & 22.5 & 23.6 & 38.5 & 40.0 & 1005.0 & 1005.5 \\
\hline Nov. 28, 2001 & 50 & 23.6 & 24.6 & 40.0 & 38.3 & 1005.5 & 1005.0 \\
\hline Nov. 28, 2001 & 60 & 25.2 & 25.4 & 34.2 & 35.0 & 1005.0 & 1004.0 \\
\hline Nov. 28, 2001 & 70 & 25.4 & 25.6 & 35.0 & 34.5 & 1004.0 & 1004.5 \\
\hline Nov. 28, 2001 & 80 & 25.6 & 25.6 & 34.5 & 38.5 & 1004.5 & 1005.0 \\
\hline Nov. 28, 2001 & 90 & 25.6 & 25.4 & 38.5 & 38.5 & 1005.0 & 1005.0 \\
\hline Nov. 28, 2001 & 100 & 25.4 & 25.2 & 38.5 & 37.2 & 1005.0 & 1005.5 \\
\hline Feb. 26, 2002 & $90,5,2$ & 29.1 & 27.5 & 28 & 28 & 987 & 984 \\
\hline Feb. 27, 2002 & 100,2 & 22.8 & 23.7 & 25 & 25 & 989 & 989 \\
\hline
\end{tabular}

\subsection{Data Set 7 -Spatial Correlation}

This data set was designed to give initial estimates of spatial correlation for the simplest of LADAR scans: replicated points along closely spaced adjacent strips. The data acquisition was very simple. The procedure involved obtaining 3 vertical scan lines with each scan repeated 10 times (see Fig. 2.7). The variables in the experiment were the vertical and horizontal angle increments $\left(0.045^{\circ}, 0.090^{\circ}\right.$, and $\left.0.180^{\circ}\right)$ - the vertical distance, $v$, between points. Twenty-five measurements per line were obtained for each line. The target was located $10 \mathrm{~m}$ from the scanner and was a sheet of plywood (4 ft x $8 \mathrm{ft}$ ) that was painted white. The data for this data set are given in Table B.6 in Appendix B. 


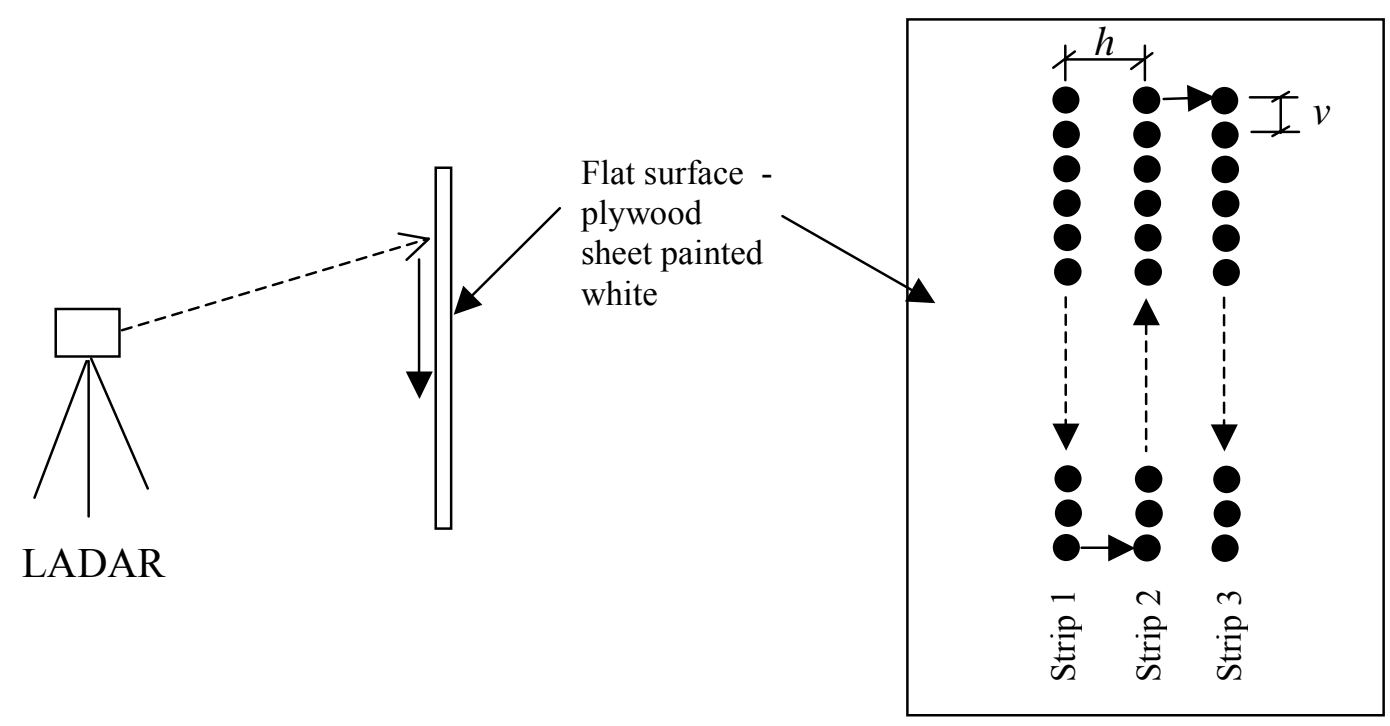

Figure 2.7. Schematic of Test Set-up.

\subsection{Data Set 8 - Autocorrelation}

The distance to the "same" point was measured 100 times to test the presence of autocorrelation. For this data set, the scanner was set up at about $20 \mathrm{~m}$ from a target. Once the scanner was set up, measurements were obtained by firing the laser and then manually recording the measurement - this procedure was repeated 100 times. The data are given in Table B.7, in Appendix B. 


\subsection{ANALYSIS AND DISCUSSION OF RESULTS}

This chapter has been organized by the examination of specific effects rather than by a sequential discussion of each data set. The effect of color is presented in Section 3.1 and the data from Data Sets 1 to 6 are included. A discussion of the effect of target color/reflectivity and distance on intensity, Data Set 5, is given in Section 3.2. The effects of angle of incidence, Data Set 5 and 6, are presented in Section 3.3, and beam divergence, Data Set 6, is discussed in Section 3.4. Discussions of autocorrelation, Data Set 8, and covariance, Data Set 7, are given in Section 3.5.

\subsection{Color}

\subsubsection{Data Set 1}

Table 3.1 gives the slopes with associated standard uncertainties from ordinary linear leastsquares fits of LADAR distance measurements versus interferometer distance measurements. In addition to the slope and its standard uncertainty, residual standard deviations from the fitted line are also reported in Table 3.1. The residual standard deviation is computed as the square root of the sum of squares of the deviations from the fitted line (residuals) divided by the degrees of freedom, $(\mathrm{N}-2)$, where $\mathrm{N}$ is the number of points and " 2 " represents the two parameter constraints (slope and intercept).

In Table 3.1, two sets of numbers are given for the slope, standard uncertainty and residual standard deviation: "Replicate" and "Averaged". The values for the column labeled "Replicate" were obtained by fitting all the data points as though they were replicates. However, the points were not true replicates because the instrument was set up once and the points were taken in quick succession without the instrument being taken down and set up again after each distance measurement. Therefore, an average of the laser measurements at each distance was also fitted as a function of the interferometer distance, and the associated statistics for the regression fits are shown under the column labeled "Averaged" in Table 3.1.

Table 3.1. Data Set 1: Slopes and Standard Uncertainties From Regression Fits.

\begin{tabular}{|l|c|c|c|c|c|c|}
\hline \multirow{2}{*}{ Color/texture } & \multicolumn{2}{|c|}{ Slope } & \multicolumn{2}{c|}{$\begin{array}{c}\text { Standard Uncertainty } \\
\text { of Slope (m) }\end{array}$} & \multicolumn{2}{c|}{$\begin{array}{c}\text { Residual Std. Dev. } \\
(\mathbf{m})\end{array}$} \\
\cline { 2 - 7 } & Replicate & Averaged & Replicate & Averaged & Replicate & Averaged \\
\hline White, smooth, not shiny & 1.0070 & 1.0070 & 0.0005 & 0.0026 & 0.1305 & 0.1354 \\
\hline White, smooth, shiny & 0.9989 & 0.9988 & 0.0001 & 0.0002 & 0.0254 & 0.0117 \\
\hline Black, smooth, not shiny & 1.0034 & 1.0032 & 0.0002 & 0.0004 & 0.0334 & 0.0145 \\
\hline Black, smooth, shiny & 1.0029 & 1.0029 & 0.0001 & 0.0004 & 0.0372 & 0.0227 \\
\hline $\begin{array}{l}\text { Dark gray, rough, not } \\
\text { shiny }\end{array}$ & 1.0032 & 1.0032 & 0.0002 & 0.0005 & 0.0394 & 0.0177 \\
\hline $\begin{array}{l}\text { Yellow, smooth, not } \\
\text { shiny }\end{array}$ & 0.9992 & 0.9992 & 0.0001 & 0.0003 & 0.0258 & 0.0173 \\
\hline Pink, smooth, not shiny & 1.0001 & 1.00005 & 0.0002 & 0.0007 & 0.0412 & 0.0360 \\
\hline Green, smooth, not shiny & 0.9993 & 0.9993 & 0.0001 & 0.0005 & 0.0294 & 0.0232 \\
\hline Green, smooth, not shiny & 0.9995 & 0.9994 & 0.0001 & 0.0005 & 0.0301 & 0.0246 \\
\hline
\end{tabular}


There are no gross differences between the slopes and standard uncertainties for replicate versus the average case. In both cases, there is no gross variation in slope and standard uncertainty across different color/texture combinations. The somewhat elevated standard uncertainty for the white, smooth, not shiny target may be because of it being the first experiment run. Variations observed (not reported here) in the intercept can be attributed to the relative nature of the distance measurements and are therefore of no interest. The replicate standard deviations consistently dominate the averaged residual standard deviations (with the exception of the aberrant white/smooth/not shiny), as we would expect. The average deviation from fit error for multiple lines should exceed that for a single (averaged) line. The replicate standard deviations exceed the manufacturer's specified accuracy of $\pm 2 \mathrm{~cm}$ for all cases, whereas the averaged are much closer to the manufacturer's specification for most cases.

Application of a formal statistical test [Sachs, 1982] for the equality of slopes of least-squares fitted lines of the replicated data, in this case very large $\mathrm{N}(\approx 2357)$ formally rejects the null hypothesis of equality of slopes. This is in agreement with the conclusion that would be drawn from comparing estimated slopes $\pm 2 \sigma$ in Table 3.1, and is, at least in part, a consequence of the large sample sizes $(\approx 275)$ associated with each of the individual line fits. Here, however, the formal test is of little direct utility. We find the more broadly applicable conclusions of the graphical analyses of range- and color dependent precisions and biases to be much more informative.

A plot of the accuracy, or bias, as a function of color and surface roughness for Data Set 1 is shown in Fig. 3.1. In Fig. 3.1, the terms "tall post" and "short post" refer to a post that was located slightly behind the target. In the case of the "tall post", the post extended above the target, while for the "short post" the post did not extend above the target. The calibration with the "short post" was undertaken to determine if the presence of the "tall post" contributed to the measurement errors. When no "tall" or "short" post is indicated, a "short" post was used.

The red dashed lines ${ }^{*}$ in Fig. 3.1 indicate the stated instrument accuracy of $\pm 2 \mathrm{~cm}$. From Fig. 3.1, several observations can be made:

1. Pink, not shiny stands out as being biased consistently (i.e., independent of distance) low with respect to the nominally "true" reading.

2. Dark gray and black targets are biased consistently high with the accuracy decreasing with increasing distance from the target. The precision of the measurements for these targets is also seen to decrease with increasing distance.

3. Green and yellow lie closer to the "truth".

4. There is a visible stratification of target types indicating an unambiguous color/roughness effect for accuracy.

\footnotetext{
"General notes: 1) Throughout this report, error is used to denote: Error = Measurement - "truth". 2) Solid/dashed red lines in figures indicate stated instrument accuracy of $\pm 2 \mathrm{~cm}$.
} 


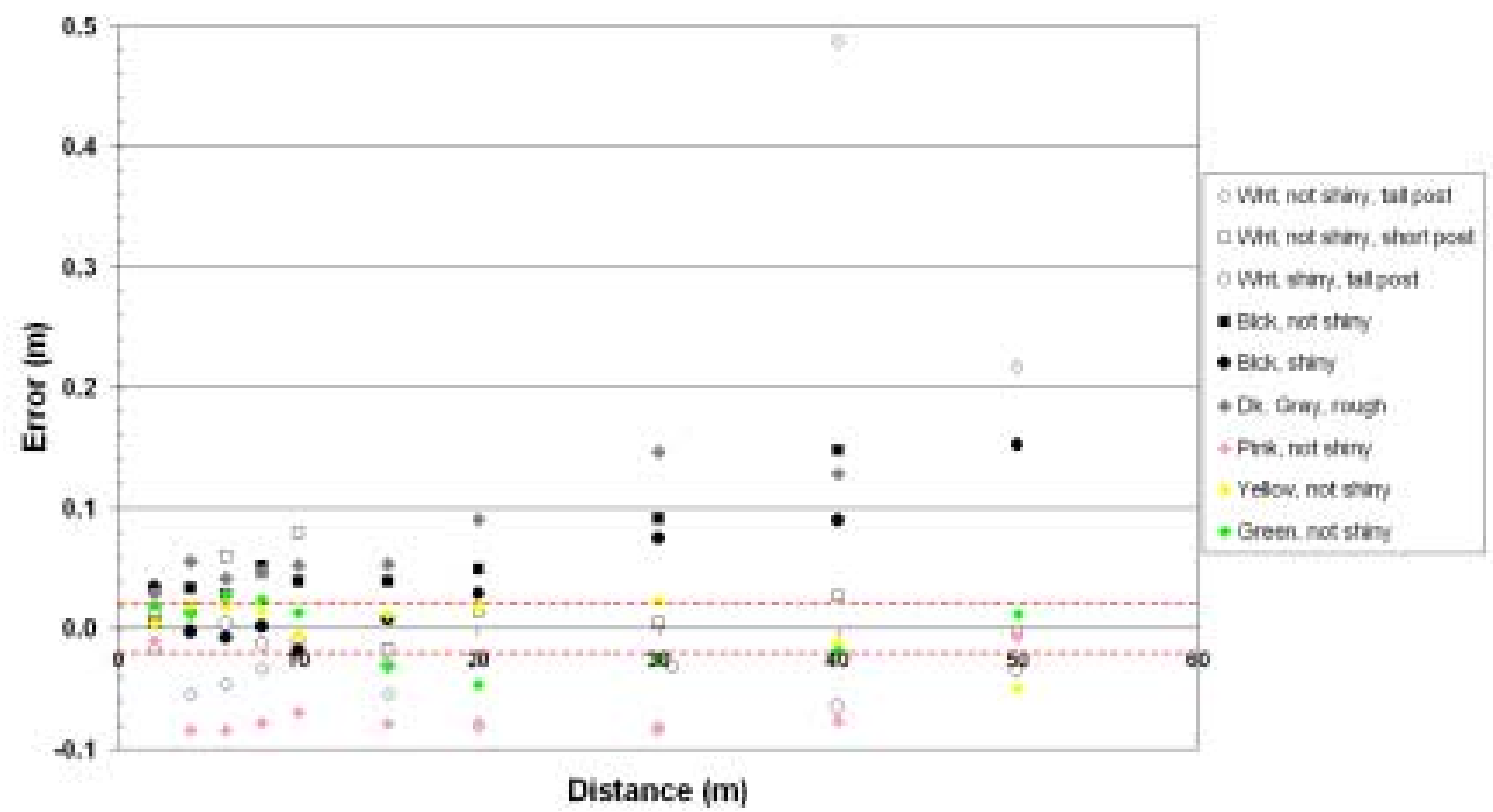

Figure 3.1 Data Set 1: Error vs. Distance $($ Error $=$ Mean laser distance - Interferometer distance $)$.

Figure 3.2 shows the scatter of the replicated laser measurements from Data Set 1 as a function of color/roughness and distance from the target. Here, standard deviation denotes the ordinary sample standard deviation ("s") computed over the replicated points at each fixed nominal distance.

Again, some patterns are readily detected in the figure. White targets are noisier in the $0 \mathrm{~m}$ to $20 \mathrm{~m}$ range, and black targets exhibit the largest variation in the $30 \mathrm{~m}$ to $50 \mathrm{~m}$ range. 


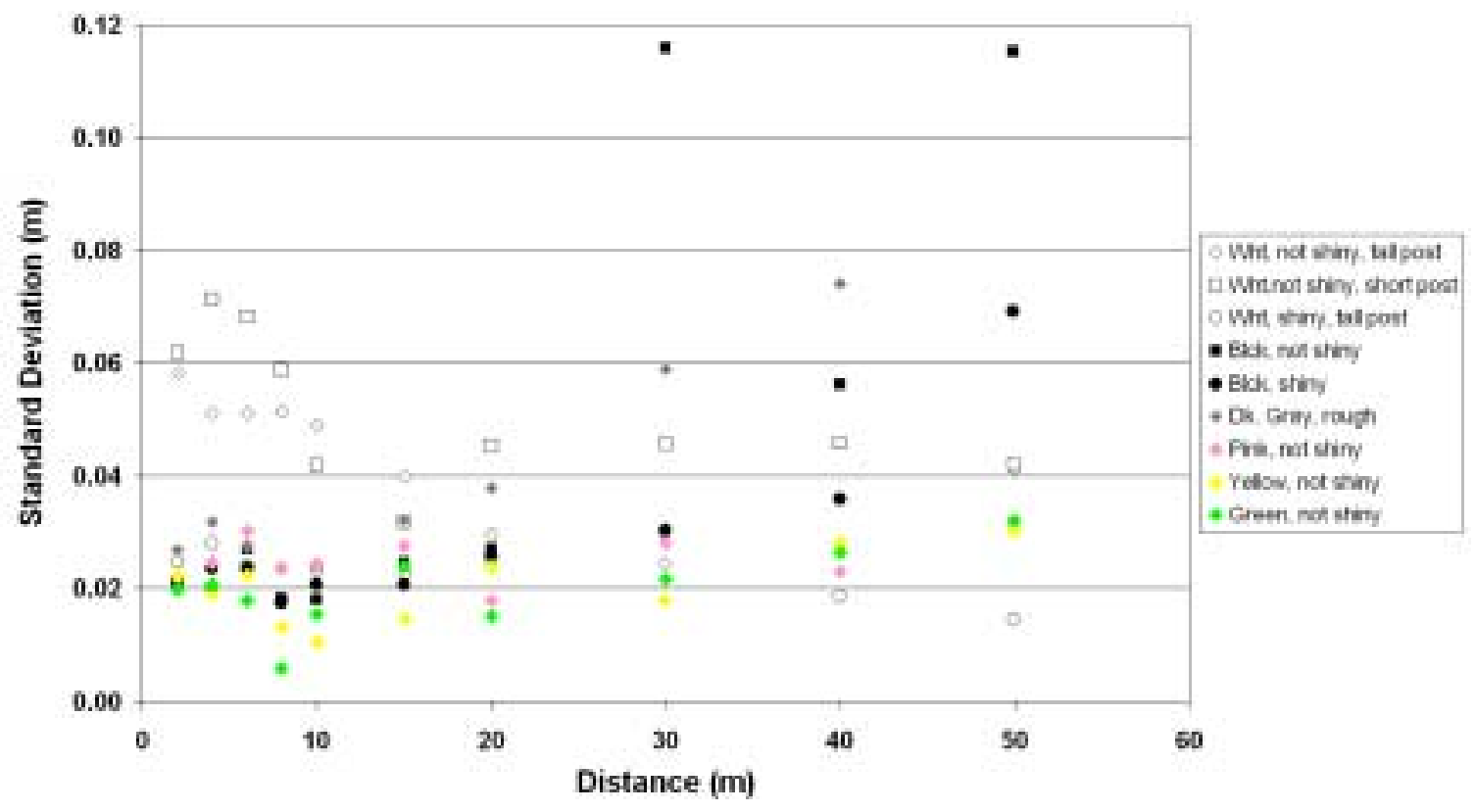

Figure 3.2. Data Set 1: Standard Deviation of Laser Measurements.

Figures 3.3a to $3.3 \mathrm{f}$ give a different representation of the data shown in Figs. 3.1 and 3.2. Accuracy and standard deviation (error bars in the figure) are plotted on the same plot for each color, separately. The observations made for the pink, yellow and green targets are also seen in Figs. 3.3d, e, and f. Some observations that are not so obvious in Figs. 3.1 and 3.2 can be appreciated in Figs. 3.3a, b, and c. In Fig. 3.3a (white target), the stratification in accuracy across the three targets represented is clear, offset only by the obvious white, not shiny, tall post highliers at $40 \mathrm{~m}$ and $50 \mathrm{~m}$. The standard deviations for the material with the higher reflectance (shiny) are smaller than for the material with less reflectance (not shiny). Figures $3.3 \mathrm{~b}$ and 3.3c (black and dark gray targets, respectively) show a clear decrease in both accuracy and repeatability as a function of increasing distance. For the black target, the one with the higher reflectance (shiny) shows a slightly lower error and greater repeatability than the target with lower reflectance.

The errors for the white, black, green, and yellow targets are seen to generally conform to the $\pm 2 \mathrm{~cm}$ specification, although the errors for the black target begin to deviate beyond $20 \mathrm{~m}$. The pink and dark gray targets, on the other hand, exceed the specification almost immediately, and throughout the entire measured range. 


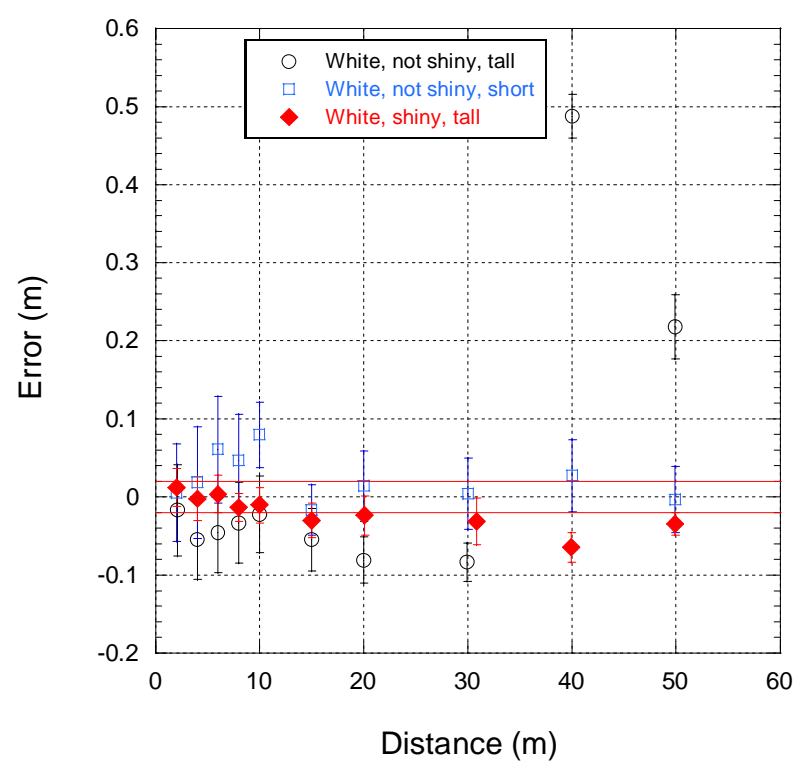

a. White

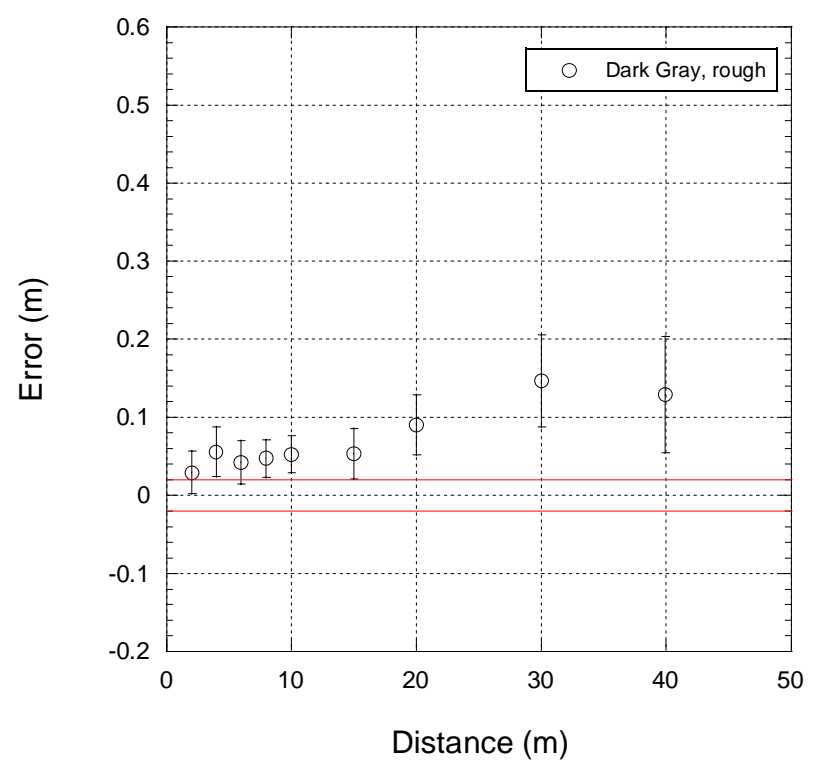

c. Dark gray

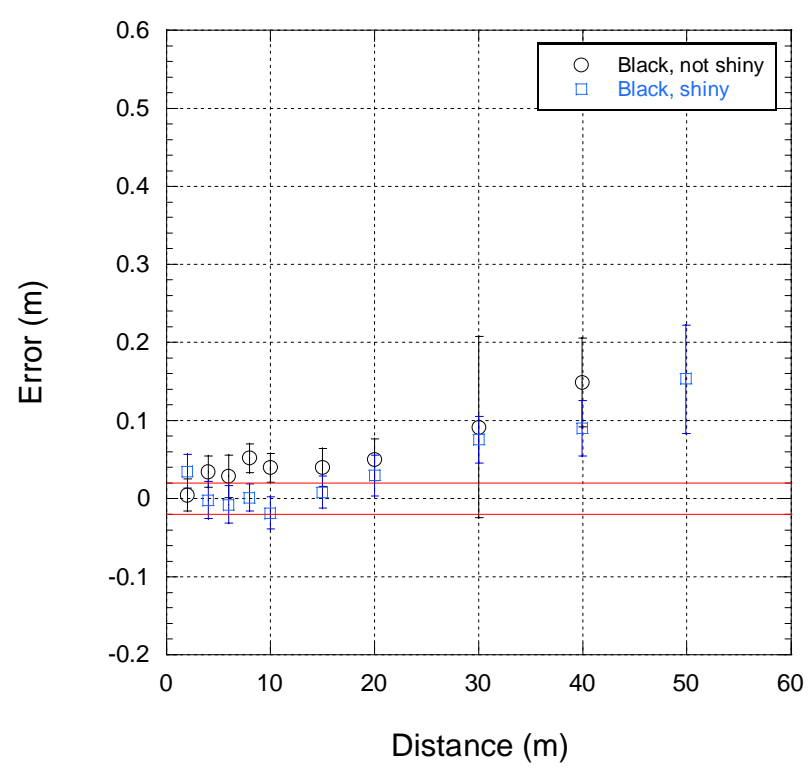

b. Black

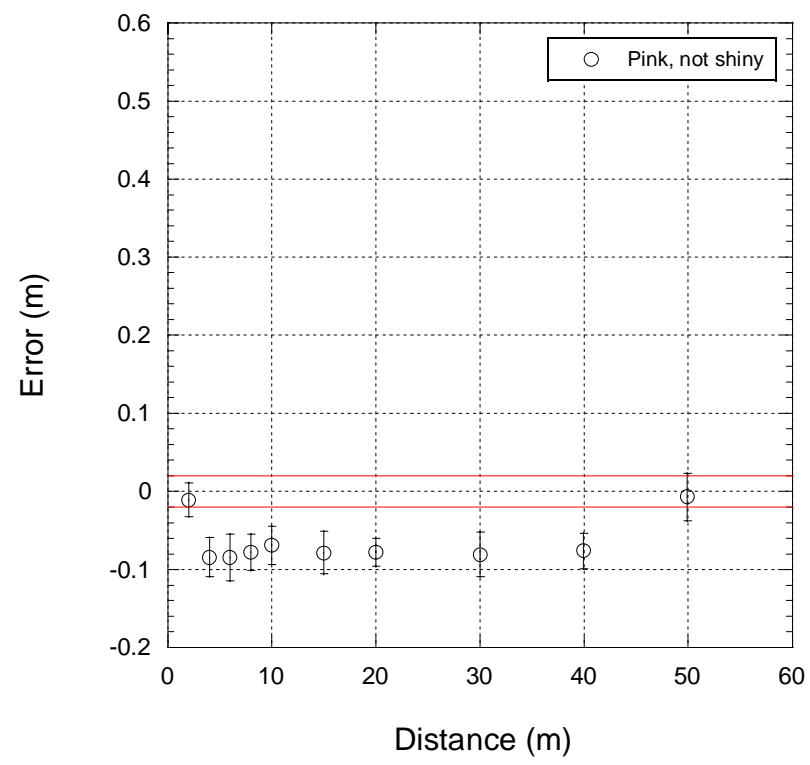

d. Pink

Figure 3.3. Data Set 1: Error Plots (Error bars $=1 \sigma)$. 


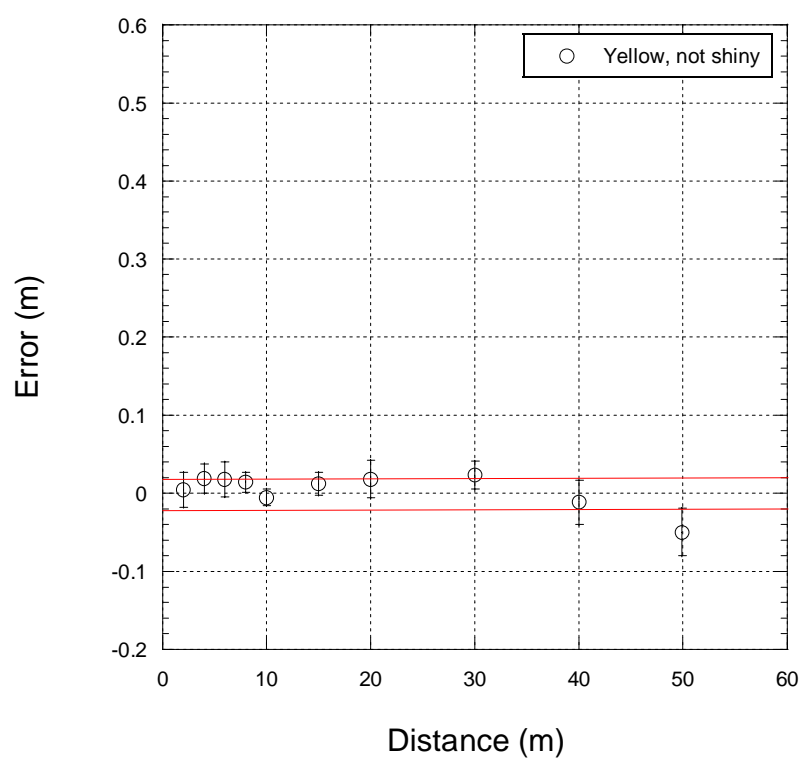

e. Yellow

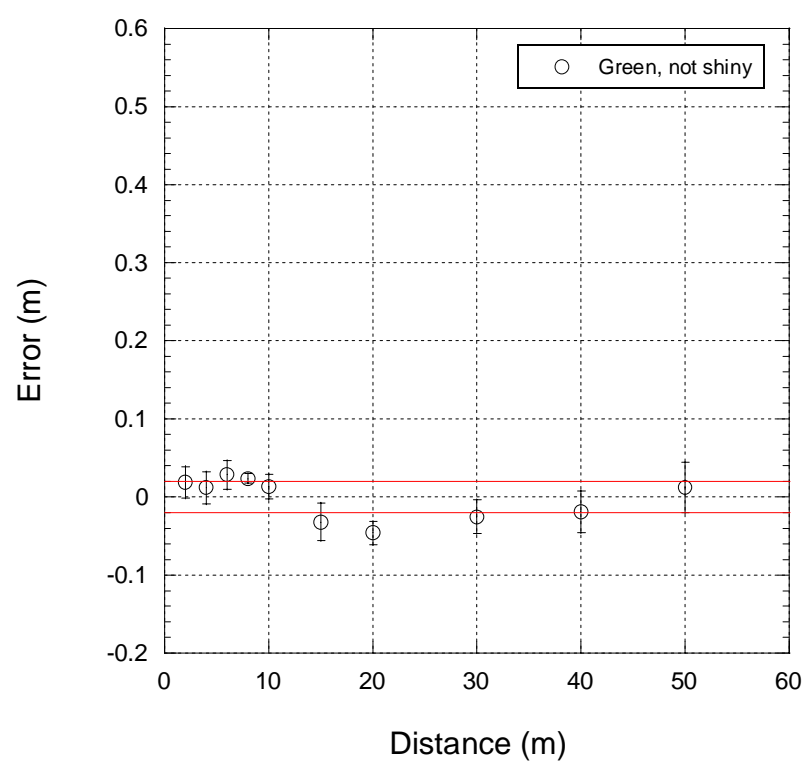

f. Green

Continue Figure 3.3. Data Set 1: Error Plots (Error bars $=1 \sigma)$.

Summary for Data Set 1: Global goodness-of-fit or precision statistics do NOT exhibit any gross color/texture-specific pattern, while localized precision estimates, in some cases, do exhibit clear patterns. Most prominent are color specific inaccuracies in distance measurement, with the black target being biased high (beyond the specifications of the instrument), white and pink targets biased low, and colors in between the two extremes giving the most accurate readings.

\subsubsection{Data Set 4}

Table 3.2 shows the coefficients and statistics for the linear regression fits for Data Set 4 - the first data set where distances ranged from $5 \mathrm{~m}$ to $150 \mathrm{~m}$. The notation "with Filter" or "Filter" refers to the case when a filter was attached to the front of the LADAR and the term "without Filter" or "No filter" refers to the case when the filter was removed. The filter was supplied by the manufacturer to reduce the output power of the LADAR for additional eye safety in indoor environments. It is expected that the data acquired with the filter attached would exhibit larger error and variability.

As seen in Table 3.2, comparison of the slopes for the black and green targets show little difference in the slopes and intercepts for the case of "with" and "without" filter. The F-test does not reject the null hypothesis of equality of slopes. However, for the black target, the residual standard deviation (RESSD) is reduced by (50 to 60) \% when the filter was removed, i.e., the data were more precise without the filter. The results for the green target are inconclusive regarding precision and the presence of the filter. 
Table 3.2 Data Set 4: Slopes and Standard Uncertainties From Regression Fits.

\begin{tabular}{|l|c|c|c|c|c|c|c|c|}
\hline \multirow{2}{*}{ Target } & \multicolumn{2}{|c|}{ Slope } & \multicolumn{2}{c|}{ Intercept (m) } & $\begin{array}{c}\text { Standard Uncertainty } \\
\text { of Slope (m) }\end{array}$ & \multicolumn{2}{c|}{ RESSD (m) } \\
\cline { 2 - 8 } & Replicate & Averaged & Replicate & Averaged & Replicate & Averaged & Replicate & Averaged \\
\hline With Filter & \multicolumn{7}{|l|}{} \\
\hline Black, not shiny & 1.0010 & 1.0010 & -0.0304 & -0.0304 & 0.00013 & 0.00029 & 0.07382 & 0.05298 \\
\hline Green, not shiny & 1.0001 & 1.0002 & 0.0252 & 0.0211 & 0.00014 & 0.00014 & 0.08347 & 0.02586 \\
\hline Without Filter \\
\hline Black, not shiny & 1.0000 & 1.0002 & 0.0266 & 0.0208 & 0.00008 & 0.00025 & 0.04568 & 0.03526 \\
\hline Green not shiny & 1.0000 & 1.0000 & 0.0153 & 0.0153 & 0.00009 & 0.00022 & 0.05090 & 0.03088 \\
\hline White, not shiny & 1.0001 & 1.0001 & 0.0286 & 0.0282 & 0.00005 & 0.00010 & 0.02918 & 0.01763 \\
\hline
\end{tabular}

Figures 3.4a and $3.4 \mathrm{~b}$ show the error as a function of distance to the target for Data Set 4 . Figure 3.4a shows the errors when no filter was attached to the LADAR. Fig. 3.4b shows the errors when the filter was attached to the LADAR.

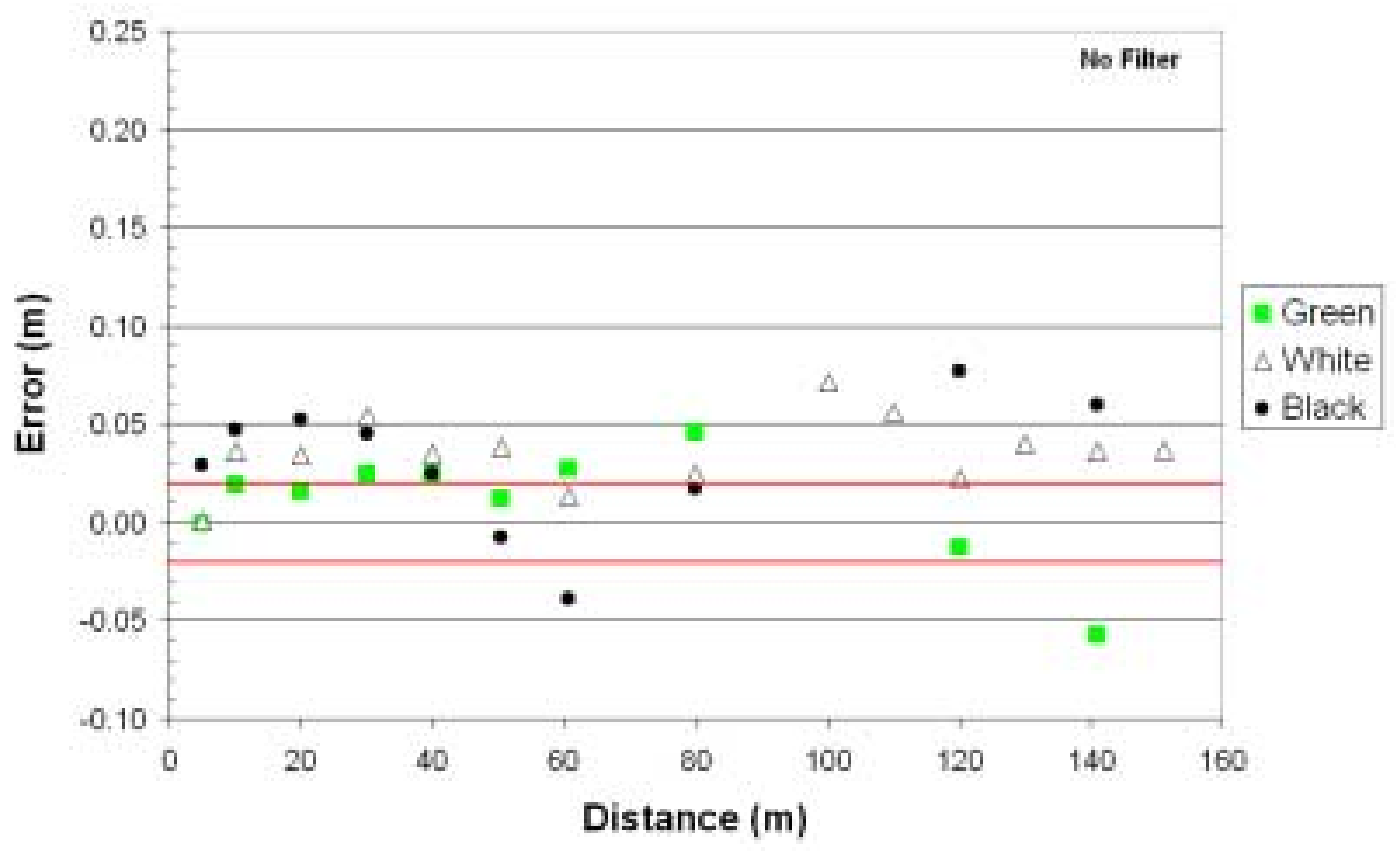

Figure 3.4a. Data Set 4: Error vs. Distance. No Filter on LADAR 


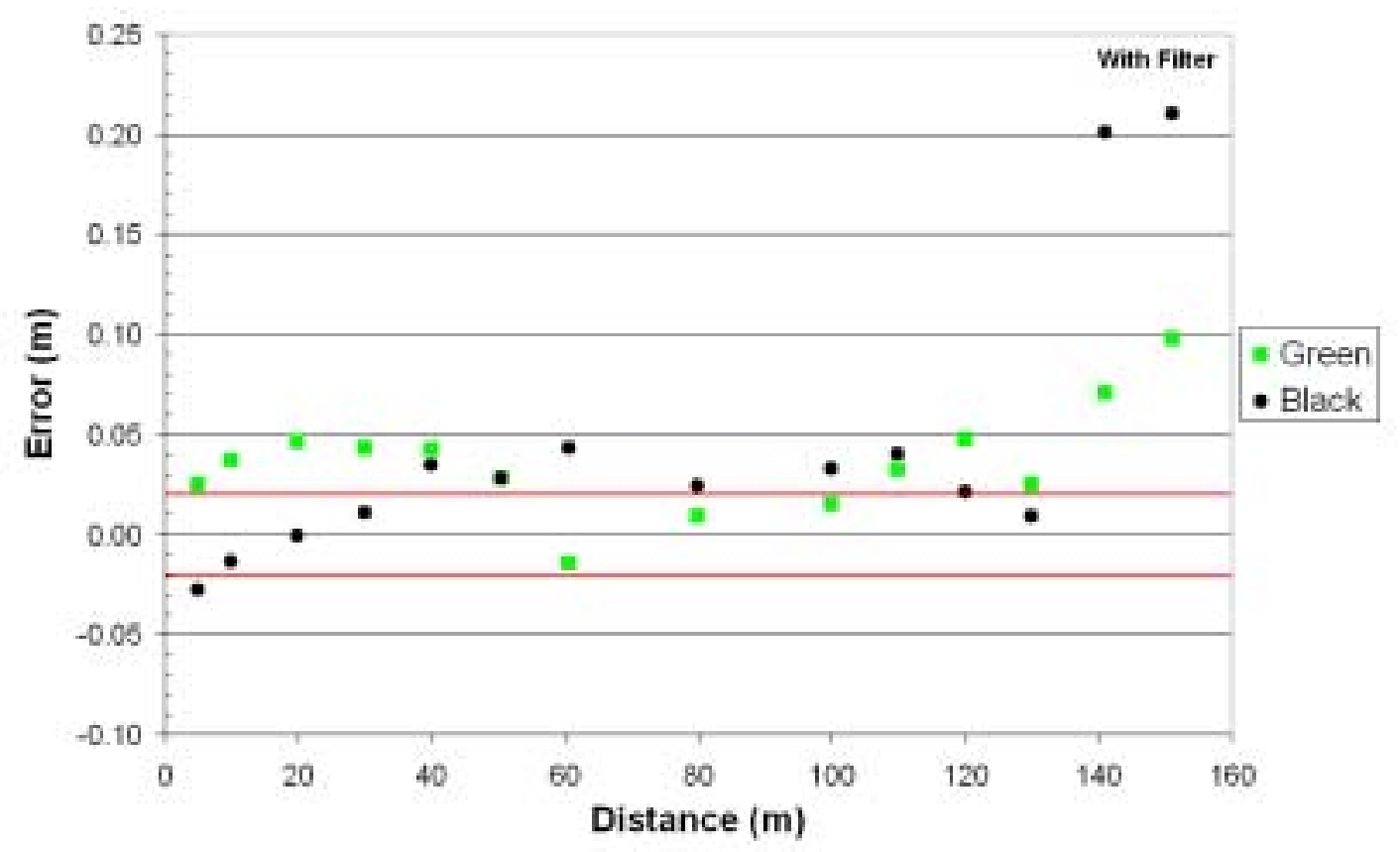

Figure 3.4b. Data Set 4: Error vs. Distance. Filter on LADAR

In the "No Filter" figure (Fig. 3.4a), there is a subtle but unmistakable stratification between green and white targets, with white errors dominating across most of the range, specifically in the $0 \mathrm{~m}$ to $50 \mathrm{~m}$ and in the $100 \mathrm{~m}$ to $160 \mathrm{~m}$ regions. The errors attributed to the black target, in contrast, meander and intermix throughout the plot.

In the "With Filter" figure (Fig. 3.4b), there is no apparent color effect, although the error increases noticeably for both colors in the $140 \mathrm{~m}$ to $160 \mathrm{~m}$ range. It appears that the combination of reduced power due to the presence of the filter and dispersion of the signal strength with distance, and a less reflective (i.e., more absorbing) target has a significant adverse affect on the accuracy at the upper range of the scanner. Since Figs. 3.4a and b have the same vertical scale, they can be compared directly. Except for the high lying points at $140 \mathrm{~m}$ and $160 \mathrm{~m}$, there is no readily apparent difference in accuracy between the filtered and unfiltered situations, contrary to expectations.

The standard deviations of the errors for Data Set 4 are given in Figs. 3.5a and 3.5b. The figures make clear that the measurements obtained with the filter are noisier that those obtained without the filter for distances of $120 \mathrm{~m}$ to $150 \mathrm{~m}$. Also, a green versus black effect is clearly visible in the filtered case, variabilities (standard deviations) for the black target dominating those of the green for almost all the distances. 


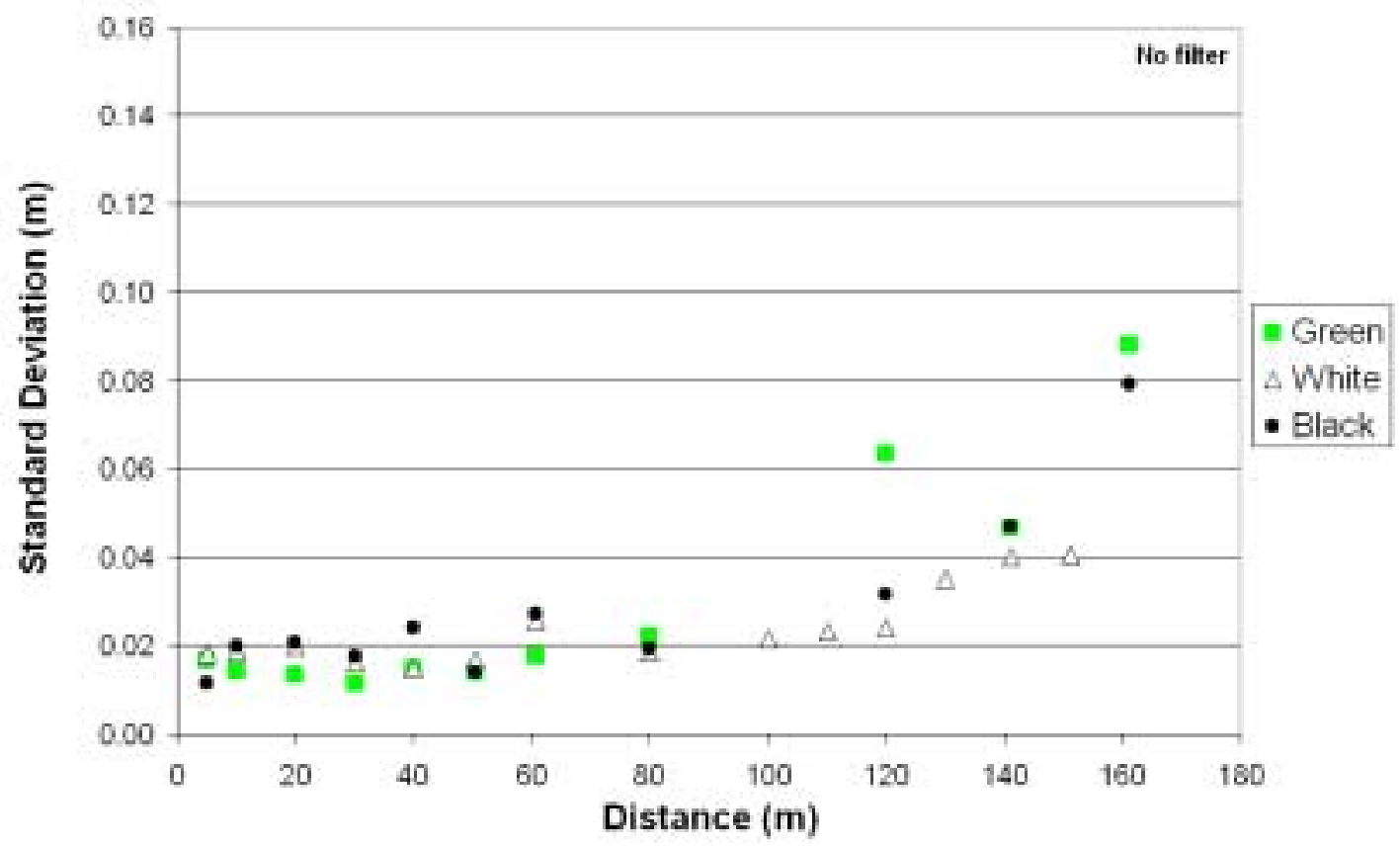

Figure 3.5a. Data Set 4: Standard Deviation of Errors. No Filter on LADAR

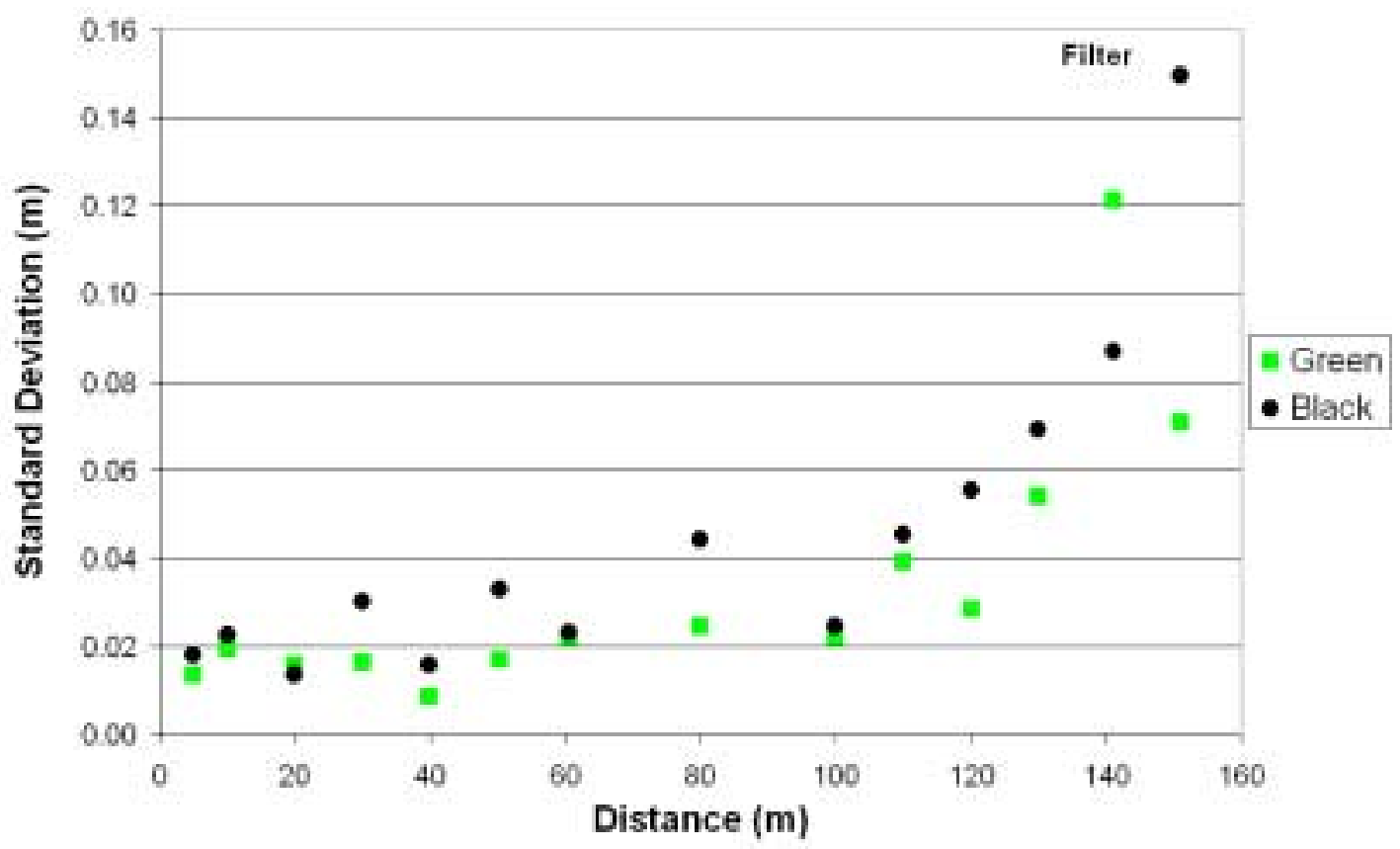

Figure 3.5b. Data Set 4: Standard Deviation of Errors.

Filter on LADAR. 
Figures 3.6a to 3.6c break out the data from Figs. 3.4 and 3.5, a color at a time. Figures 3.6 reinforce the conclusions from Figs. 3.5, except that in addition, these figures make clear that (1) in all cases, variability increases with increased distance, and the consequent tendency for points to fall outside the specification $\pm 2 \mathrm{~cm}$ and (2) this heteroscedastic effect - in the case of length metrology - is especially pronounced for the filtered black and green cases.

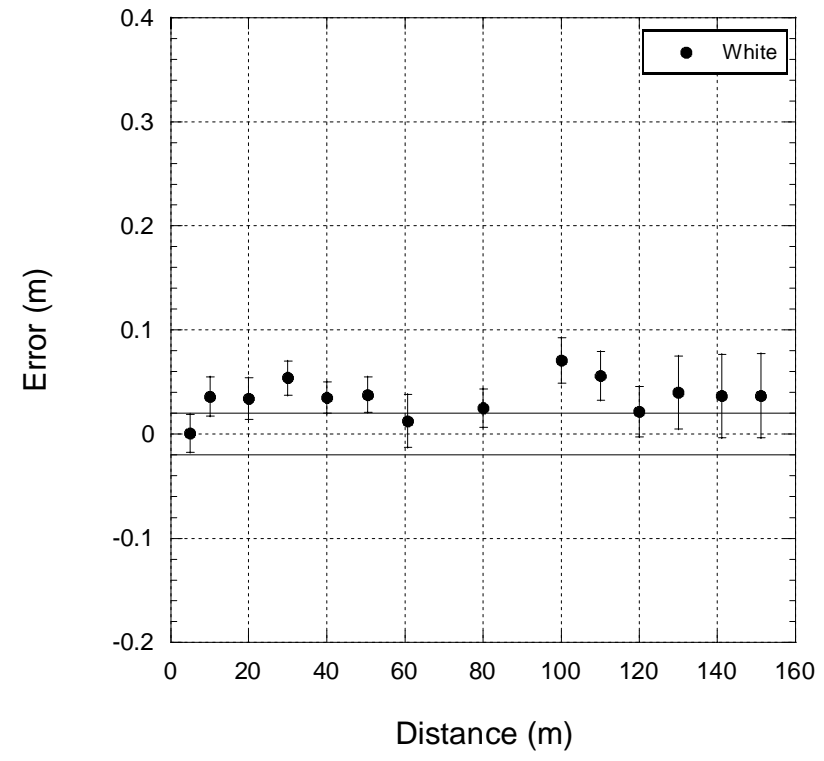

a. White

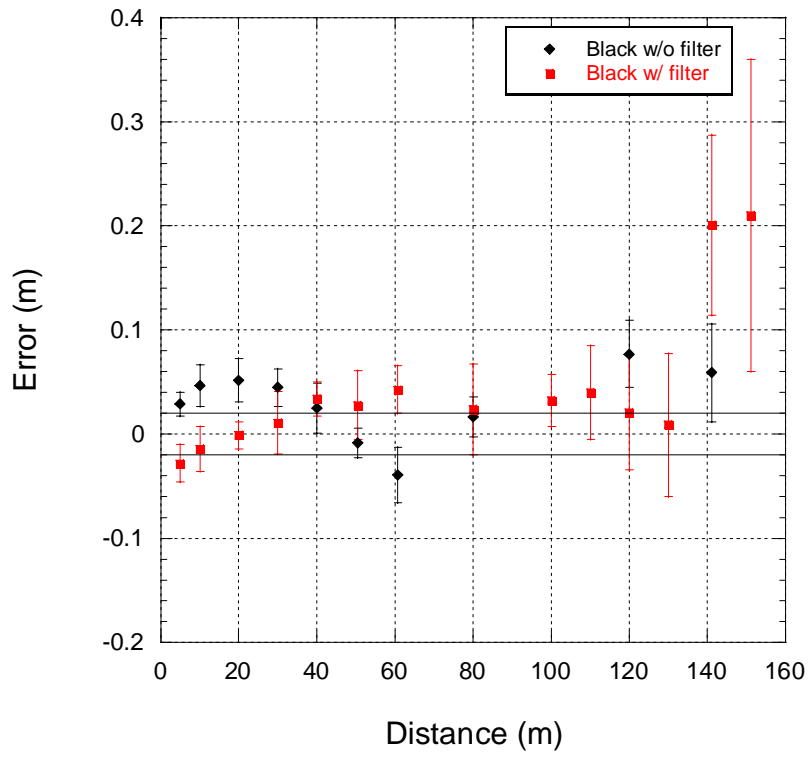

b. Black

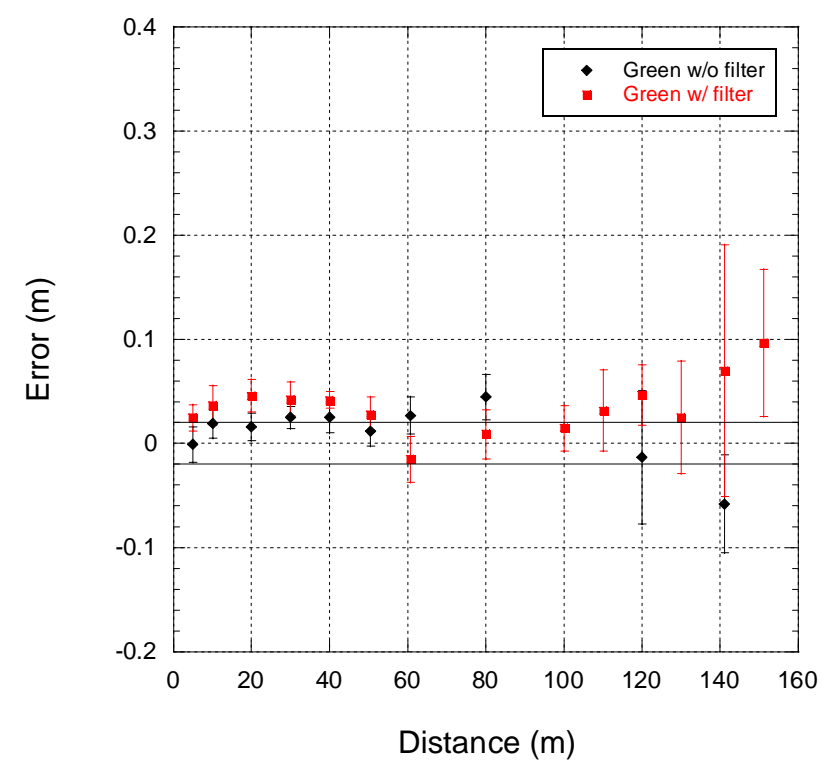

c. Green

Figure 3.6. Data Set 4: Error Plots (Error bars $=1 \sigma$ ) 
Summary for Data Set 4: No marked filter or color effect is observed in the global line statistics. But subtle effects are observed with respect to localized precision and statistics, and significant effects with respect to accuracy. The presence of the filter significantly increases the error and noise for the black target (least reflective) at the upper range of the scanner.

\subsubsection{Data Set 5}

The locations at which the data for Data Sets 1 to 3 were collected did not allow for the calibration of the maximum range of the LADAR. The locations at which the data for Data Sets 4 and 5 were collected did allow for calibration of the maximum range of the LADAR. However, the location for Data Set 5 was felt to be more environmentally controlled than the location of Data Set 4 (see Section 2.4). Therefore, for these reasons in addition to the fact that more experienced was gained by the time the data for Data Set 5 was collected, the data in Data Set 5 is considered to be more reliable for the evaluation of range accuracy and precision as a function of color and distance.

The coefficients for the regression fits for Data Set 5 are shown in Table 3.3. As with the previous data sets, there are no gross differences among the slopes and standard uncertainties of the slope except for the LDP, $60^{\circ}$ target which has a standard uncertainty that is significantly higher than the rest, although, the formal F-test does reject the null hypothesis of the equality of slopes. The residual standard deviations for the black, white, and silver shiny targets are within about $10 \%$ of each other and are slightly over the specified accuracy of $\pm 2 \mathrm{~cm}$. The residual standard deviation for the LDP, $0^{\circ}$ target is about $25 \%$ greater that the average of the black, white, and silver, shiny targets. As expected, the residual standard deviation increased for increased angle of incidence - significantly greater than the $\pm 2 \mathrm{~cm}$ specified limits. Further comparisons of the LDP targets will be made in Section 3.3 (angle of incidence). Discussions in this section will generally include only the first four targets listed in Table 3.3 since are they are comparable, i.e, the angle of incidences for these four targets are $0^{\circ}$.

Table 3.3. Data Set 5: Slopes and Standard Uncertainties from Regression Fits.

\begin{tabular}{|l|c|c|c|c|c|c|c|c|}
\hline \multirow{2}{*}{ Target } & \multicolumn{2}{|c|}{ Slope } & \multicolumn{2}{c|}{ Intercept } & \multicolumn{2}{c|}{$\begin{array}{c}\text { Standard Uncertainty } \\
\text { of Slope (m) }\end{array}$} & \multicolumn{2}{c|}{$\begin{array}{c}\text { RESSD } \\
(\mathbf{m})\end{array}$} \\
\cline { 2 - 9 } & Replicate & Averaged & Replicate & Averaged & Replicate & Averaged & Replicate & Averaged \\
\hline Black & 1.0001 & 1.0001 & -0.0104 & -0.0110 & 0.00007 & 0.00016 & 0.03875 & 0.02734 \\
\hline White & 0.9995 & 0.9995 & 0.0210 & 0.0200 & 0.00007 & 0.00018 & 0.03501 & 0.03040 \\
\hline Silver, Shiny & 1.0001 & 1.0001 & -0.0254 & -0.0256 & 0.00004 & 0.00005 & 0.02159 & 0.00878 \\
\hline LDP, $0^{\circ}$ & 0.9987 & 0.9987 & 0.1128 & 0.1132 & 0.00007 & 0.00021 & 0.03862 & 0.03563 \\
\hline LDP, $45^{\circ}$ & 0.9997 & 0.9996 & -0.0200 & -0.0193 & 0.00009 & 0.00027 & 0.04646 & 0.04464 \\
\hline LDP, $60^{\circ}$ & 1.0001 & 1.0000 & 0.0010 & 0.0029 & 0.00013 & 0.00041 & 0.06778 & 0.06844 \\
\hline
\end{tabular}

The errors and standard deviations of the errors for Data Set 5 are shown in Figs. 3.7 and 3.8, respectively. In Fig. 3.7, the high reflectance of the LDP target induces significant error in the distance measurement for distances of $20 \mathrm{~m}$ or less. Overall, the LDP trace appears to linearly increase through the entire range. The large errors for the LDP target in the shorter distances $(0 \mathrm{~m}$ to $50 \mathrm{~m})$ are likely a result of the detector being saturated by the strong return signal of the 
highly reflective material. Furthermore, these errors are biased low, that is, the target appears closer than in actuality. These observations were also noted by Collier [1998]. Reflectance is seen in this case to contribute directly to inaccuracy in the distance measurements.

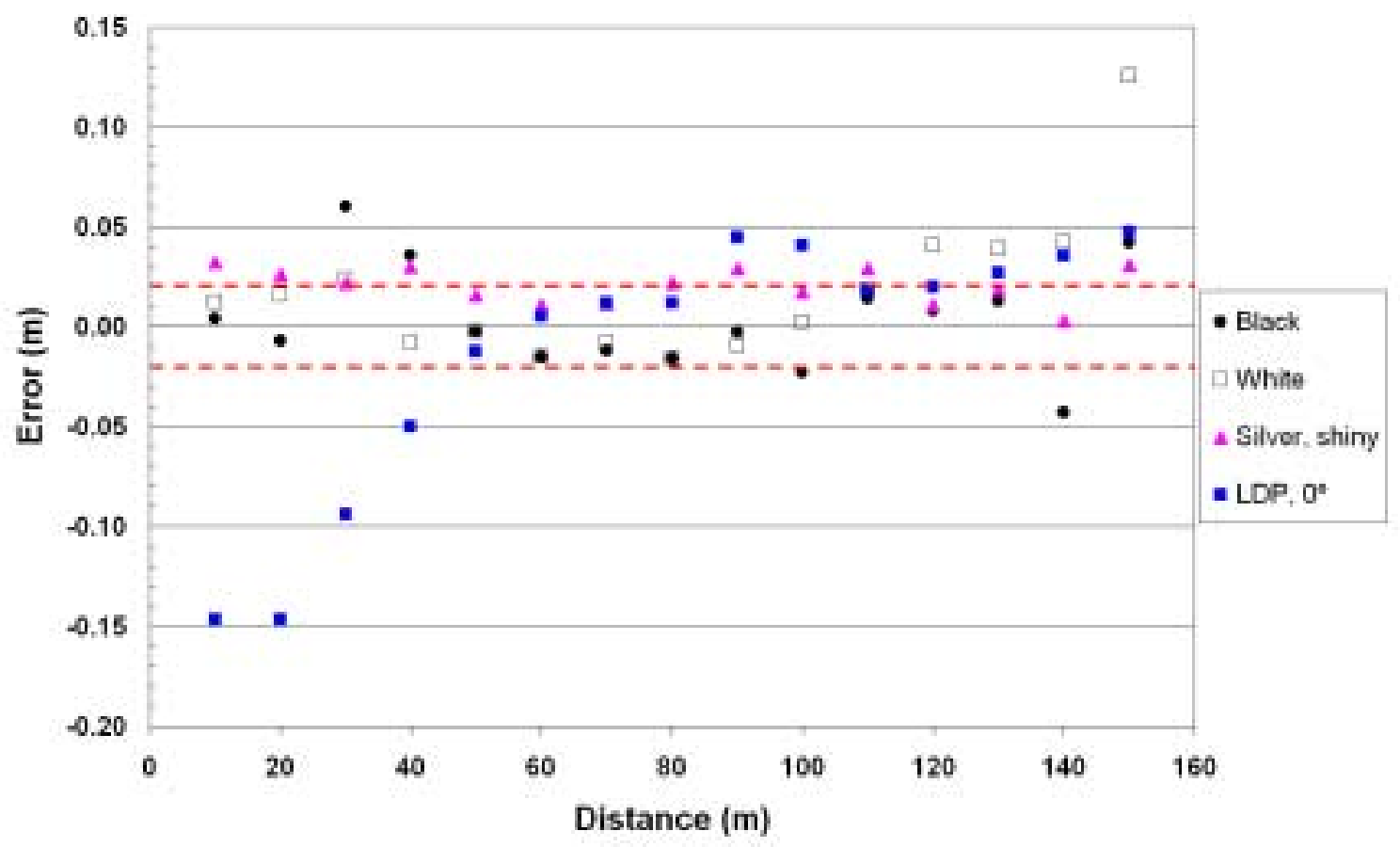

Figure 3.7. Data Set 5: Error vs. Distance.

$($ Error $=$ Mean laser distance - total station distance $)$.

There are no obvious patterns for repeatability, or standard deviations of the replicated measurements, for most of the targets in Fig. 3.8: the values for white, silver shiny and LDP targets do not stand out in terms of increased or decreased or stratified noisiness. Black, however, does pull apart from the other three in the $80 \mathrm{~m}$ to $150 \mathrm{~m}$ range: the black measurements for this range are distinctly noisier than those for the other three targets. 


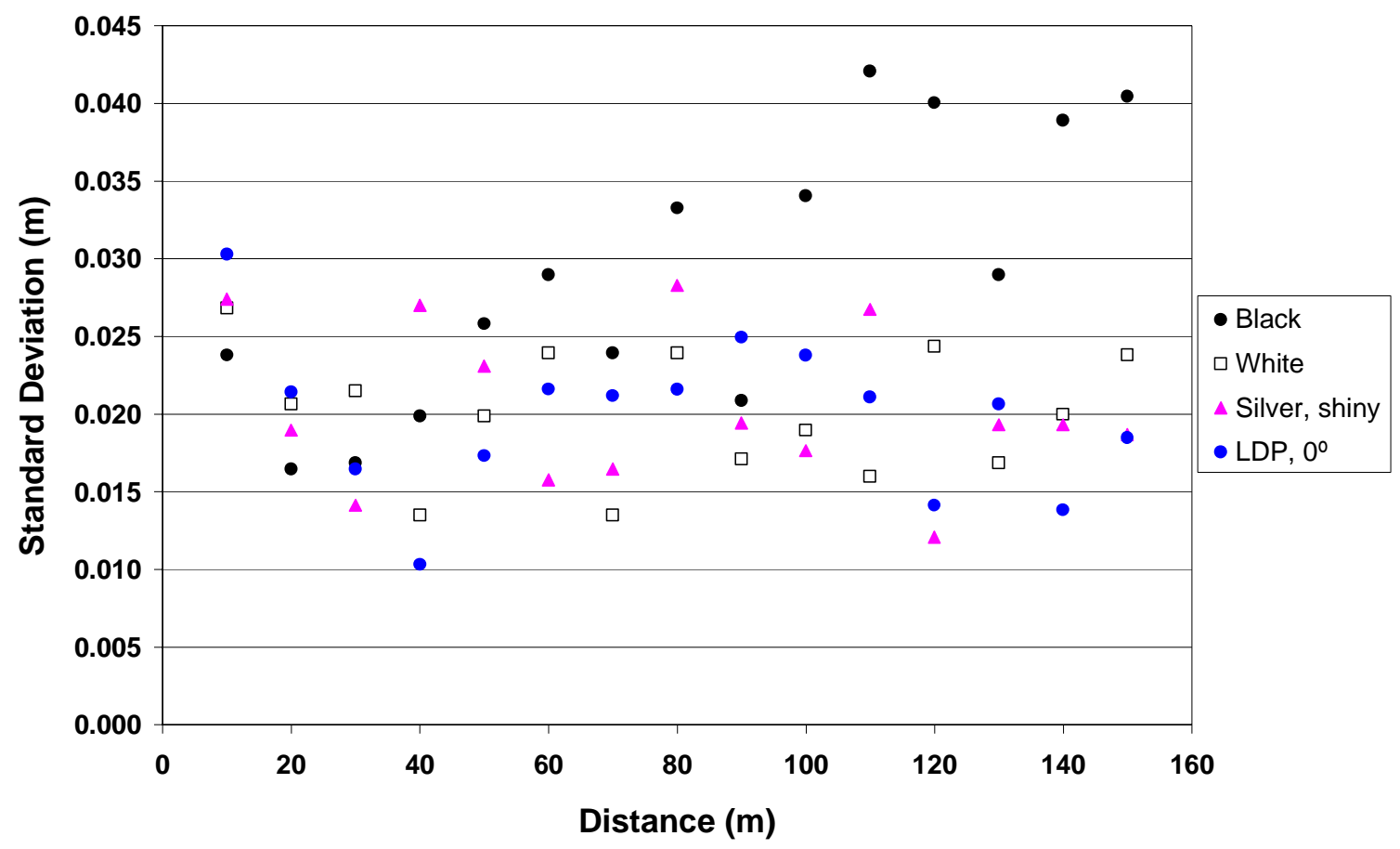

Figure 3.8. Data Set 5: Standard Deviation of Error of Laser Measurements.

Figures 3.9a to 3.9d break out the data from Figs. 3.7 and 3.8, a color at a time. The figures display clearly the degraded accuracy of the LDP target at $0 \mathrm{~m}$ to $40 \mathrm{~m}$, and again, the roughly linear rise of the LDP error from negative to positive bias. While the sliver and black targets results appear relatively tame, the accuracy for the white target decreases steadily for distances beyond $120 \mathrm{~m}$. Again, the error bars of Fig. 3.9a clearly show that the black target is noisier than the other targets in the $80 \mathrm{~m}$ to $150 \mathrm{~m}$ range. This decrease in precision with increase distance was also noted in Data Set 4; however, this phenomenon was noted for all targets - black, white, and green - and not just for the black target as is the case in Data Set 5. 


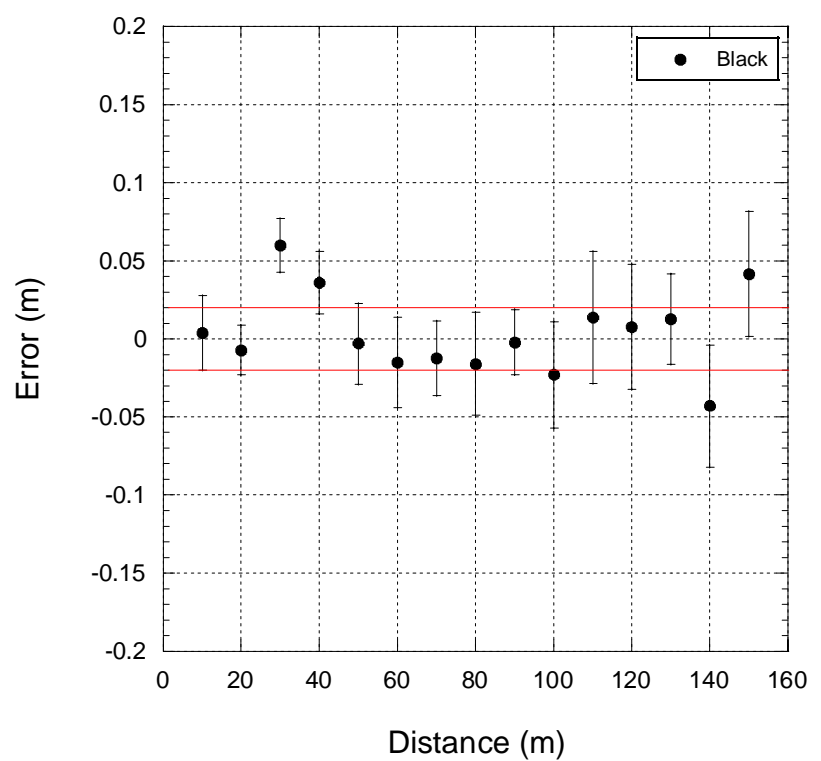

a. Black

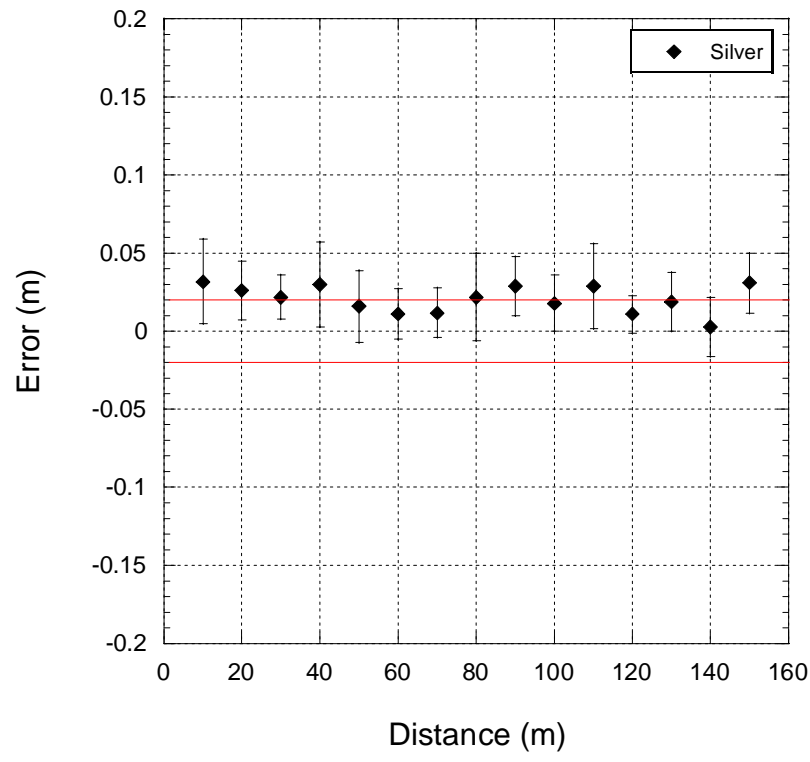

c. Silver

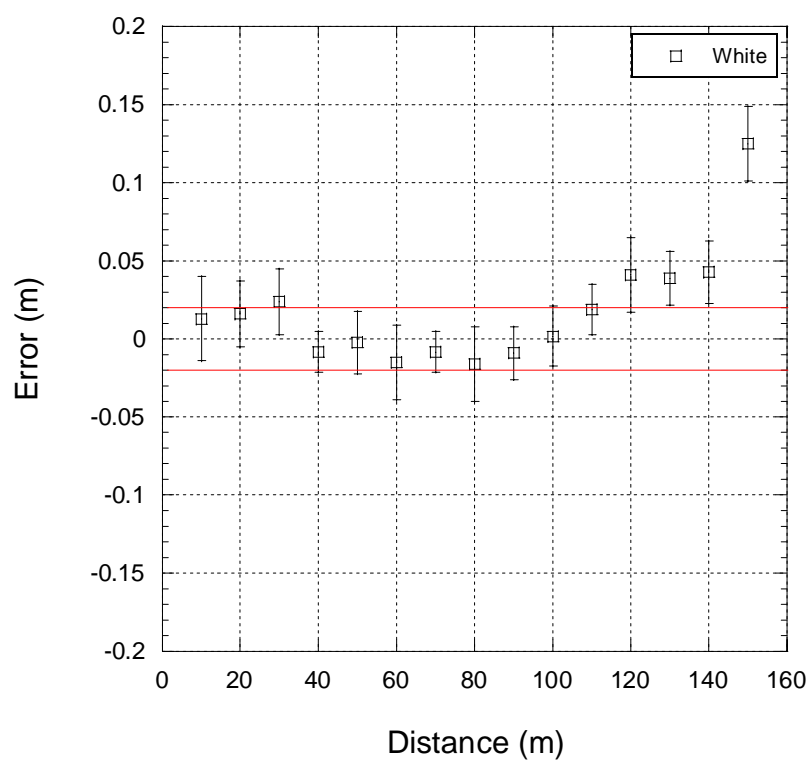

b. White

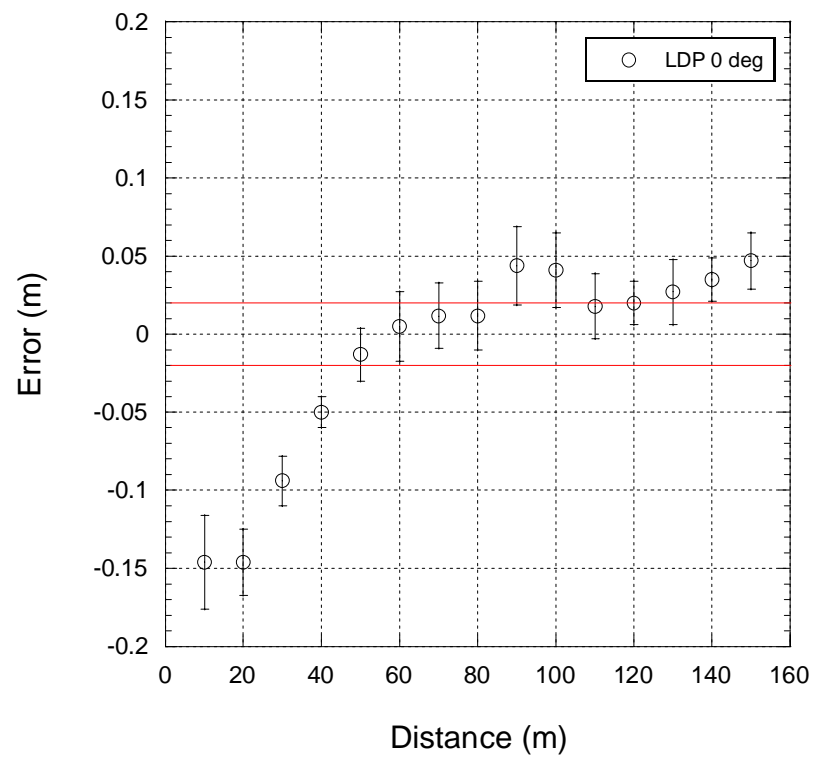

d. LDP, $0^{\circ}$

Figure 3.9. Data Set 5: Error Plots (Error bars $=1 \sigma)$.

Summary of Data Set 5: Effects appear both in the consideration of localized precision statistics and overall residual error, and very noticeable effects are seen on accuracy, particularly in the case of the LDP target. It is clear that reflectance properties can have very real effects on the accuracy of the distance measurements. 


\section{$\underline{3.1 .4}$ Combined Data Sets}

Figures $3.10 \mathrm{a}$ and $3.10 \mathrm{~b}$ show the errors and standard deviations for white and black targets cumulated from Data Sets 1 to 6. From Fig. 3.10a, there is no pronounced black versus white effect although about half of the data fall outside the specified instrument accuracy. In Fig. 3.10b, a clear pattern can be discerned. The measurements of white targets are noisier in the $0 \mathrm{~m}$ to $50 \mathrm{~m}$ range while the black targets are noisier in the $60 \mathrm{~m}$ to $150 \mathrm{~m}$ range with a linear increase above the $100 \mathrm{~m}$ range. In fact, it appears that the repeatability for the white targets goes roughly linearly down across the range of distances presented by the data while the repeatability for the black targets goes approximately linearly up.

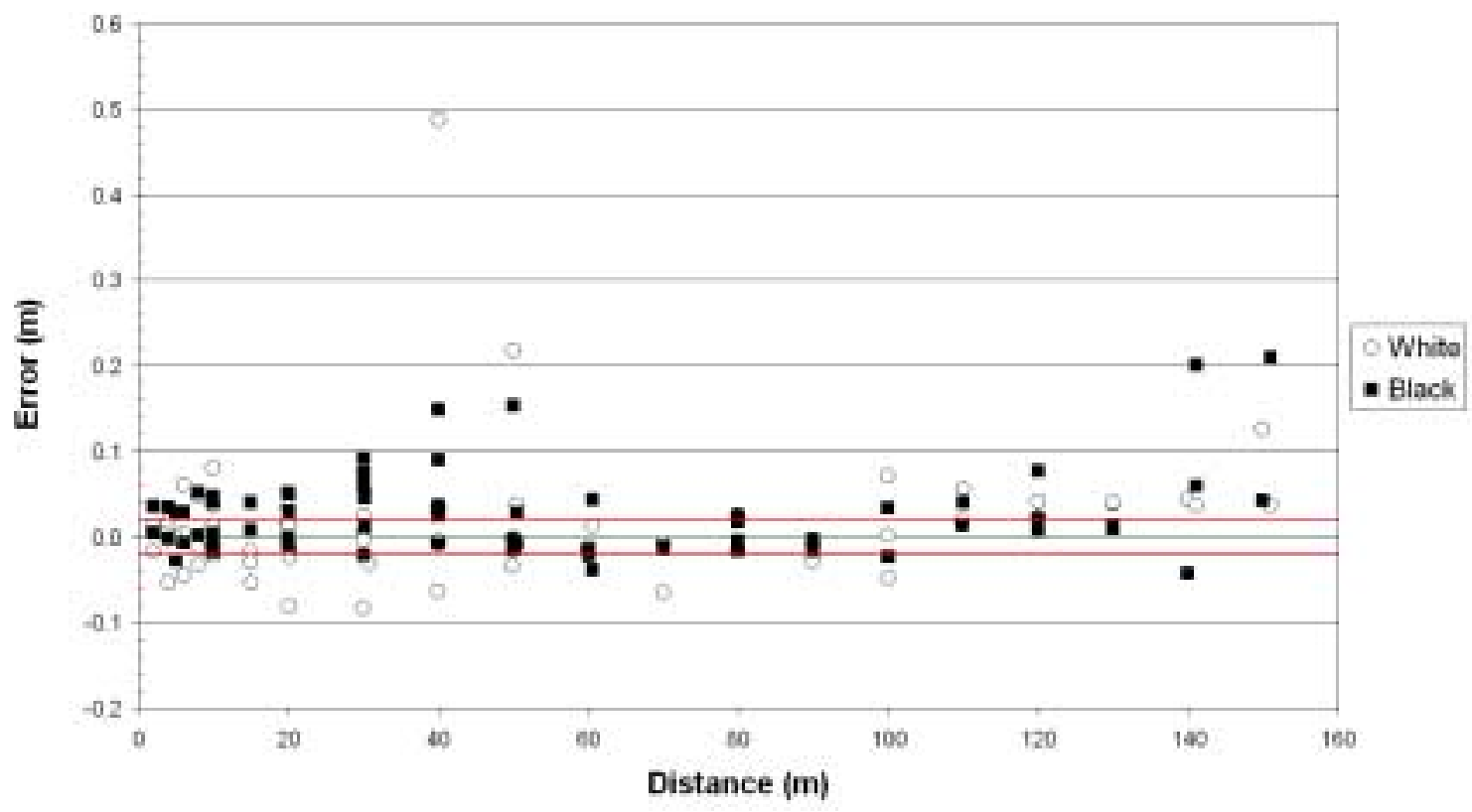

Figure 3.10a. Combined Errors for White and Black

Targets from Data Sets 1 to 6. 


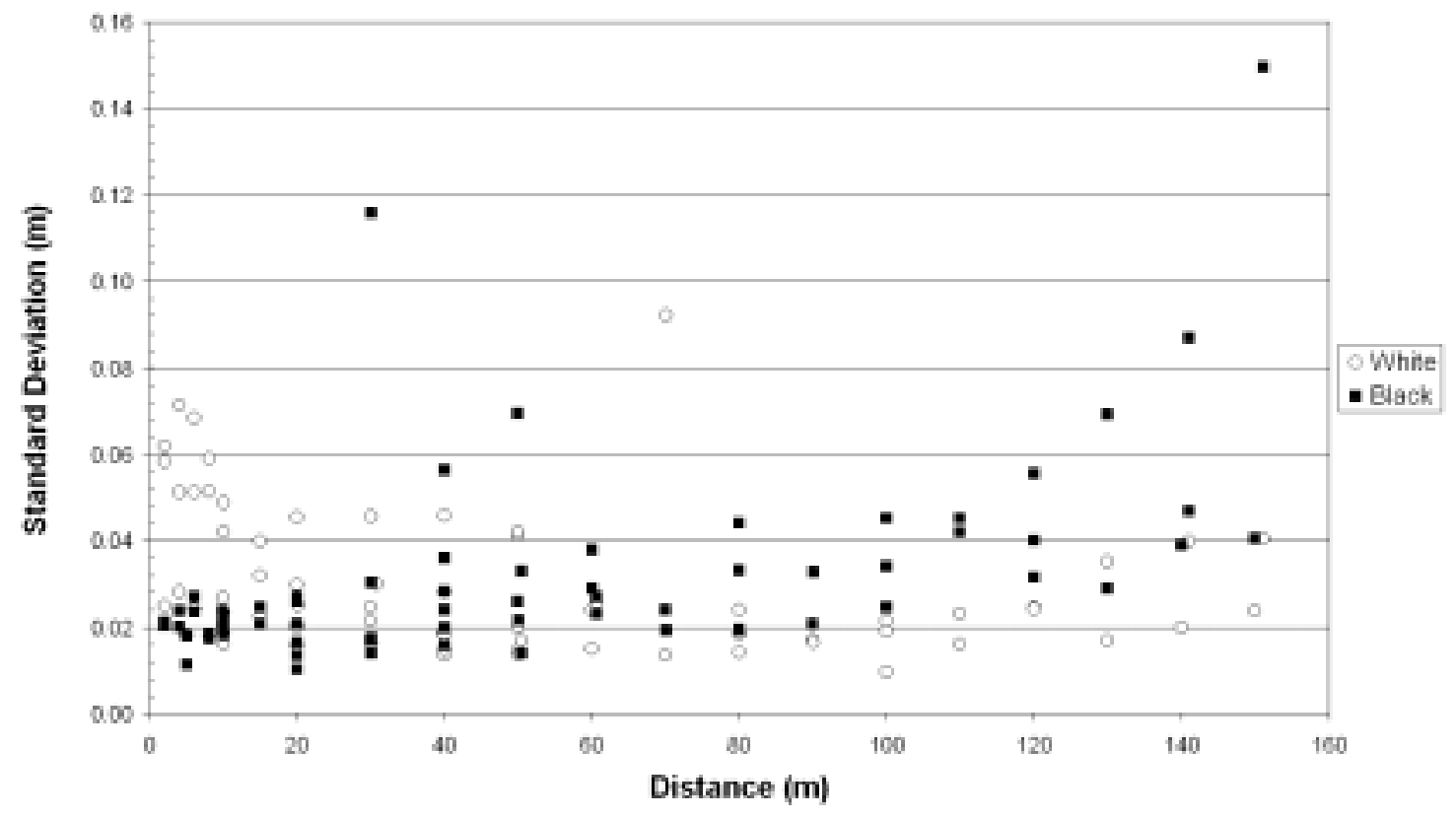

Figure 3.10b. Standard Deviations for White and Black

Targets from Data Sets 1 to 6.

\subsection{Intensity}

A plot of the intensity versus distance to the target is shown in Fig. 3.11. As seen in Fig. 3.11, the intensity values for the LDP target at $0^{\circ}$ are consistently high -200 to 250 over the entire range of the scanner - and are easily distinguishable from the other targets. As expected, the intensity values drop off when the target is turned $45^{\circ}$ away from the scanner - the intensity values for the LDP target at $45^{\circ}$ are very similar to those for the shiny silver target at $0^{\circ}$ and one could be mistaken for the other. At an angle of incidence of $60^{\circ}$, the intensity values for a LDP target would be indistinguishable from values for the white target. Although stratification of the intensities for white and LDP at $60^{\circ}$ targets can be seen in Fig. 3.11, it would be hard to determine the color/reflectance of the object struck for each point in a scene. For example, an intensity value of about 90 could be for a point off an LDP target rotated at $60^{\circ}$ at $120 \mathrm{~m}$ or a white target at $80 \mathrm{~m}$ or a black target at $20 \mathrm{~m}$. Thus, without additional information, one would not be able to determine if the target was white, black or LPD rotated at $60^{\circ}$. Also, as mentioned in Section 2.2.4, the intensity values also depend on the lighting conditions and would likely be different in bright sunlight or in shadow. 


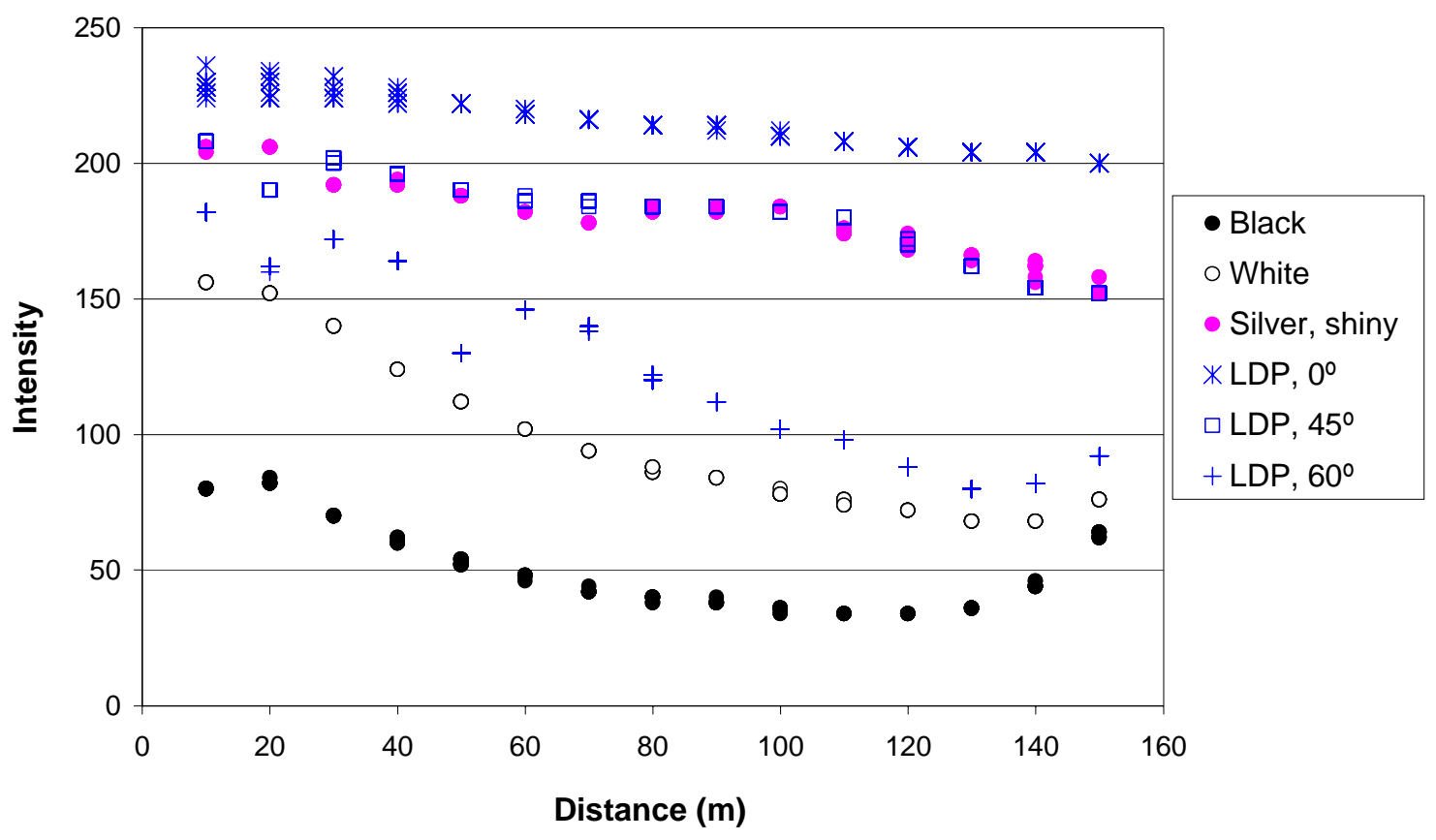

Figure 3.11. Data Set 5: Intensity vs. Distance.

In Fig. 3.11, the intensity values for the black, white, and LDP at $60^{\circ}$ targets are increasing at distances of $140 \mathrm{~m}$ and $150 \mathrm{~m}$ which is contrary to what would be expected. This increase may be attributed to the contribution of the white wall behind the target at the longer distances. The wall was located about $160 \mathrm{~m}$ from the scanner.

The intensities from Data Set 6 are plotted in Figs. 3.12a to 3.12c. The actual intensity values from this data set are very similar to those from Data Set 5 and show the same sort of trends for the white, black, and silver targets, with the silver target displaying the most pronounced stratification, especially at angle of incidences of $0^{\circ}$ and $10^{\circ}$. In Fig. $3.12 \mathrm{c}$, the intensities for the targets at higher angles of incidence appear to increase as the distance increases. This increase in intensity is due to the fact that a larger portion of the beam is being reflected back off the white wall behind the target at the higher angles of incidence. 


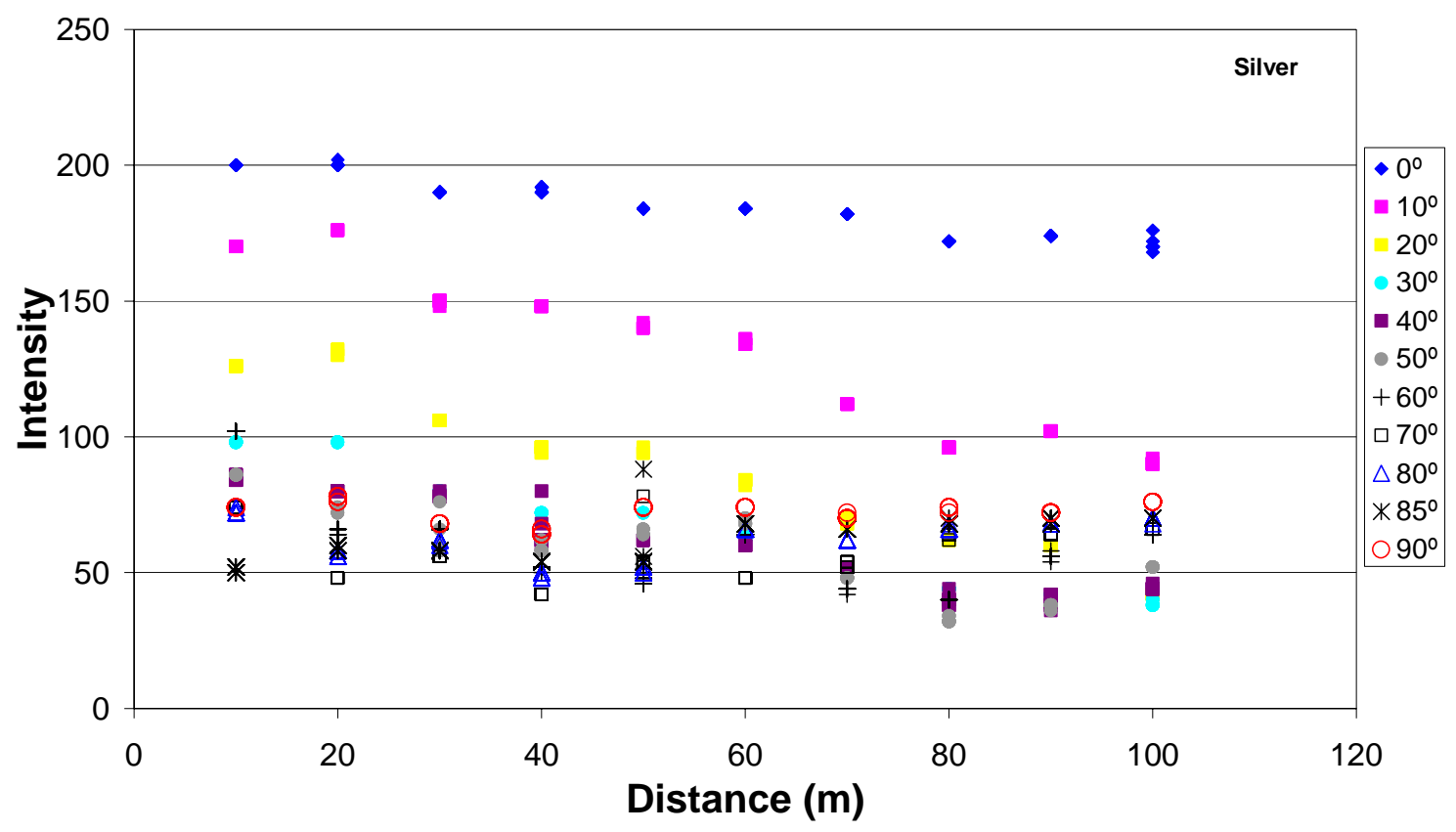

Figure 3.12a. Data Set 6: Silver Target Intensity as a Function of Distance and Angle of Incidence.

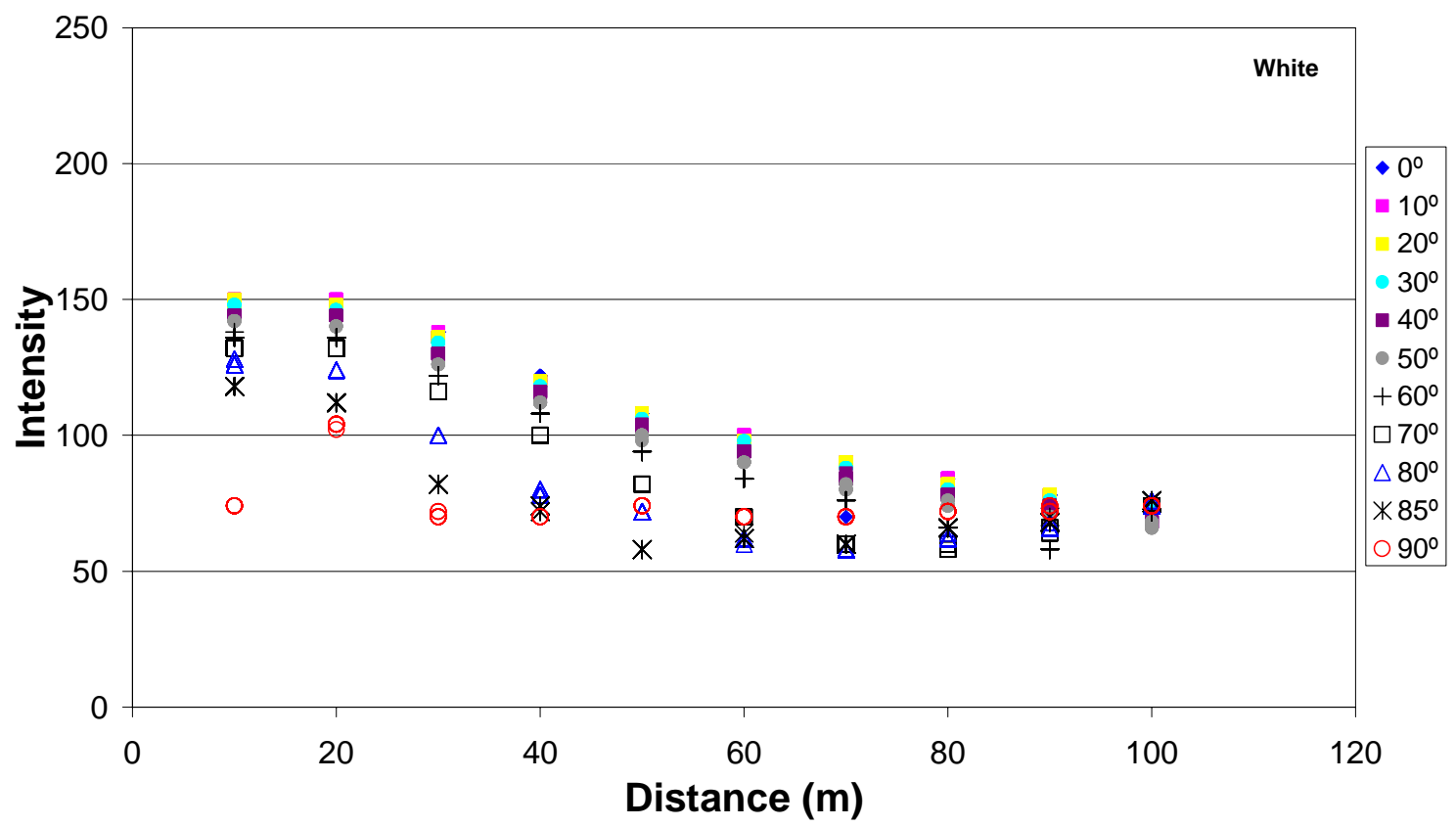

Figure 3.12b. Data Set 6: White Target Intensity as a Function of Distance and Angle of Incidence. 


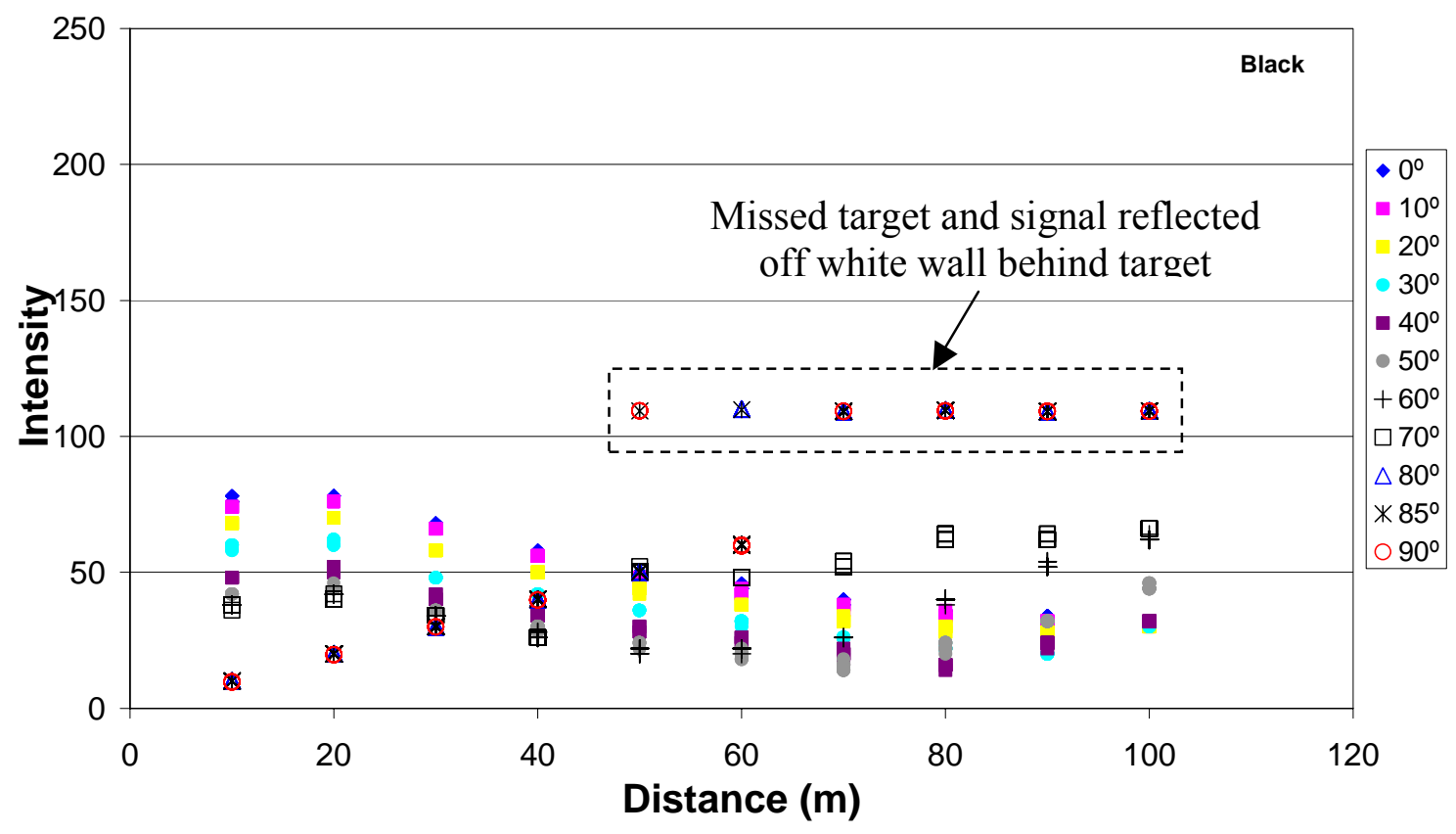

Figure 3.12c. Data Set 6: Black Target Intensity as a Function of Distance and Angle of Incidence.

Summary for Intensity data: The objective of these experiments was to find a material which would yield consistently high intensity values for the entire range of the scanner. A clear effect is seen on intensity as a function of reflectance - targets with high reflectance yield higher intensities. However, the intensities degrade with increasing distance. On the other hand, the LDP targets at $0^{\circ}$ and $45^{\circ}$ show less degradation than the other targets and yield consistently high intensities for all distances - making these targets easily distinguishable from other targets. These results are encouraging and indicate that the LDP material could potentially be used to fabricate bar codes or tags that can be read by a LADAR.

\subsection{Angle of Incidence}

\section{$\underline{3.3 .1 \quad \text { Data Set } 6}$}

For each rotation angle (angle of incidence) and for each target color, the measured distances were plotted against the actual distance $(10 \mathrm{~m}$ to $100 \mathrm{~m})$. For each of these plots, least squares regression lines were fitted through the points. The standard deviations of the residuals (averaged case) from the fitted line are given in Table 3.4 and plotted in Fig. 3.13. In Fig. 3.13, the maximum scale of the $\mathrm{Y}$-axis was set to $1 \mathrm{~m}$, and the residual standard deviations for the silver target at $80^{\circ}$ and $85^{\circ}$ rotation are greater than $1 \mathrm{~m}$ and are therefore not shown. 
Table 3.4. Residual Standard Deviation from Linear Regression.

\begin{tabular}{|c|c|c|c|}
\hline \multirow{2}{*}{$\begin{array}{c}\text { Rotation Angle } \\
\left(\mathbf{(}^{\mathbf{}}\right.\end{array}$} & \multicolumn{3}{|c|}{ Residual Standard Deviation (m) } \\
\cline { 2 - 4 } & White & Black & Silver \\
\hline 0 & 0.0348700 & 0.0268980 & 0.0206252 \\
\hline 10 & 0.0221700 & 0.0290150 & 0.0238604 \\
\hline 20 & 0.0227700 & 0.0362710 & 0.0667554 \\
\hline 30 & 0.0220900 & 0.0404220 & 0.3260688 \\
\hline 40 & 0.0230400 & 0.0765750 & 0.1136327 \\
\hline 50 & 0.0422800 & 0.0598770 & 0.4613150 \\
\hline 60 & 0.0642100 & 0.0592890 & 0.5549653 \\
\hline 70 & 0.0548600 & 0.0769410 & 0.6897836 \\
\hline 80 & 0.0480000 & 0.0793640 & $\mathbf{2 . 5 9 3 3 8 3 0}$ \\
\hline 85 & 0.0632800 & 0.0853410 & $\mathbf{5 . 0 0 5 6 0 1 0}$ \\
\hline 90 & 0.0769480 & 0.1027240 & 0.0945438 \\
\hline
\end{tabular}

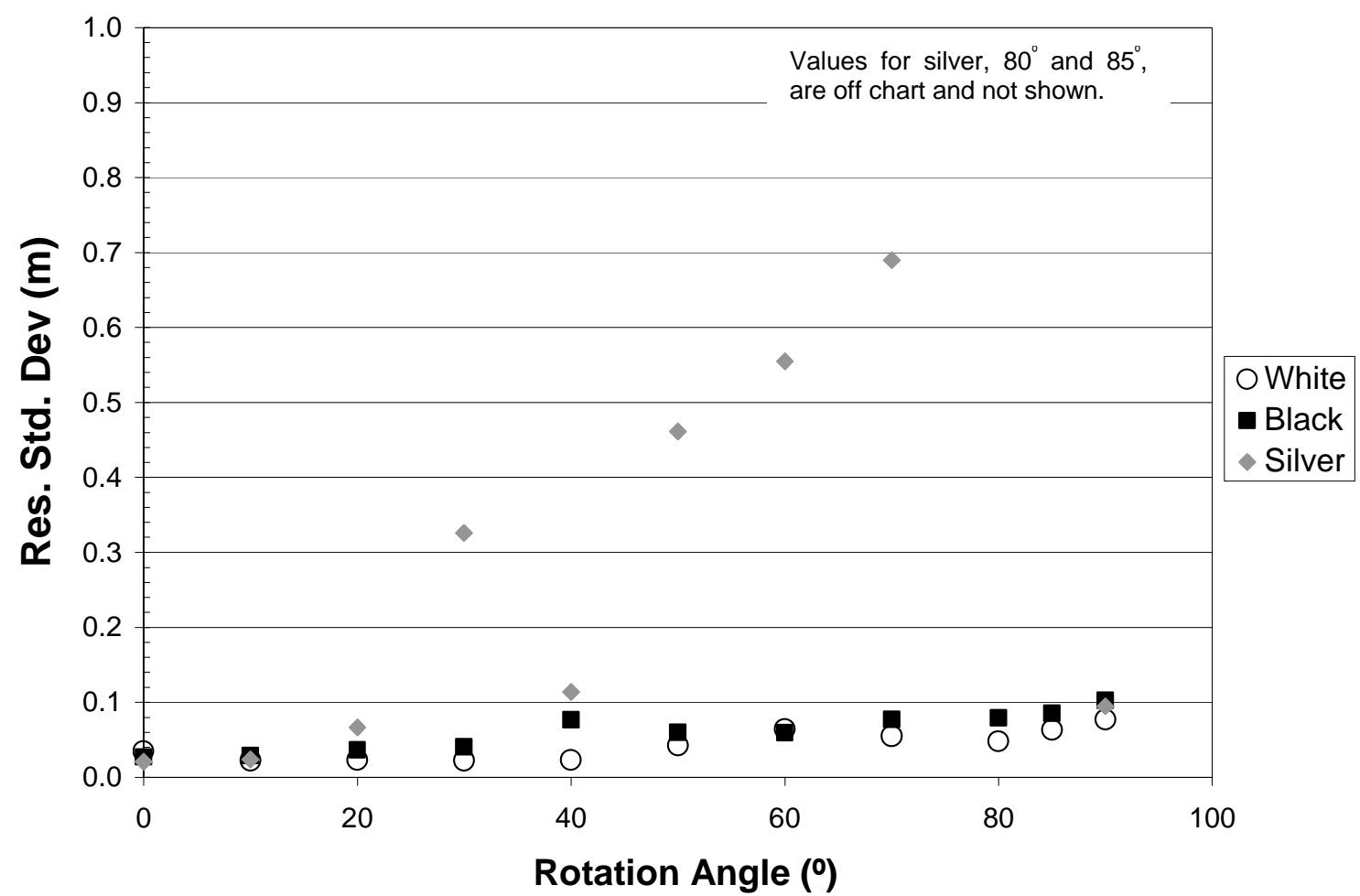

Figure 3.13. Data Set 6: Residual Standard Deviation.

From Table 3.4 and Fig. 3.13, it is seen that for the white target, the residual standard deviation exceeded the nominal instrument accuracy for angles of incidence greater than $40^{\circ}$. Slightly degraded performance is noted for the black and silver targets. For incidence angles greater than $10^{\circ}$, the residual standard deviations for the silver and black targets are greater than the stated accuracy of the instrument $( \pm 2 \mathrm{~cm})$. However, the residual standard deviations for the silver 
target are significantly greater than those for the black target for angles of incidence between $30^{\circ}$ and $85^{\circ}$ (see discussion later in this section).

As seen in Table 3.5, it appears that the number of misses depends on the color of the target with the black target (least reflective) sustaining the most misses followed by the silver and then the white. A "miss" is defined as a laser measurement equal to $109 \mathrm{~m} ; 109 \mathrm{~m}$ is the distance from the LADAR to the wall behind target. In the case of the black target, the strength of the return signal was insufficient or below a threshold value required to trigger the detector whereas the strength of the return signal off the wall behind the target was sufficient, i.e., the black target absorbed more energy that the white wall. For the black target, partial misses (less than 10 repetitions) began occurring at angle of incidence of $50^{\circ}$ to $60^{\circ}$ for a distance of $80 \mathrm{~m}$. Total misses (10 repetitions) began occurring at angles of incidence greater than $70^{\circ}$ for distances of $70 \mathrm{~m}$ and $80 \mathrm{~m}$ and $50^{\circ}$ for distances of $90 \mathrm{~m}$ and $100 \mathrm{~m}$. 
Table 3.5. Number of Target Misses.

\begin{tabular}{|c|c|c|c|c|c|c|c|c|c|c|c|}
\hline \multirow{2}{*}{$\begin{array}{l}\text { Distance } \\
\text { (m) }\end{array}$} & \multicolumn{11}{|c|}{ Angle of Incidence $\left(^{\circ}\right)$} \\
\hline & $\mathbf{0}$ & 10 & 20 & 30 & 40 & $\mathbf{5 0}$ & 60 & 70 & 80 & 85 & 90 \\
\hline \multicolumn{12}{|l|}{ Black } \\
\hline 10 & 0 & 0 & 0 & 0 & 0 & 0 & 0 & 0 & 0 & 0 & 0 \\
\hline 20 & 0 & 0 & 0 & 0 & 0 & 0 & 0 & 0 & 0 & 0 & 0 \\
\hline 30 & 0 & 0 & 0 & 0 & 0 & 0 & 0 & 0 & 0 & 0 & 0 \\
\hline 40 & 0 & 0 & 0 & 0 & 0 & 0 & 0 & 0 & 0 & 0 & 0 \\
\hline 50 & 0 & 0 & 0 & 0 & 0 & 0 & 0 & 0 & 0 & $\mathbf{1}$ & 10 \\
\hline 60 & 0 & 0 & 0 & 0 & 0 & 0 & 0 & 0 & 10 & 1 & 0 \\
\hline 70 & 0 & 0 & 0 & 0 & 0 & 0 & 0 & 10 & 10 & 10 & 10 \\
\hline 80 & 0 & 0 & 0 & 0 & 0 & 5 & 9 & 10 & 10 & 10 & 10 \\
\hline 90 & 0 & 0 & 0 & 0 & 0 & 10 & 10 & 10 & 10 & 10 & 10 \\
\hline 100 & 0 & 0 & 0 & 0 & 1 & 10 & 10 & 10 & 10 & 10 & 10 \\
\hline \multicolumn{12}{|l|}{ Silver } \\
\hline 10 & 0 & 0 & 0 & 0 & 0 & 0 & 0 & 0 & 0 & 0 & 0 \\
\hline 20 & 0 & 0 & 0 & 0 & 0 & 0 & 0 & 0 & 0 & 0 & 0 \\
\hline 30 & 0 & 0 & 0 & 0 & 0 & 0 & 0 & 0 & 0 & 0 & 0 \\
\hline 40 & 0 & 0 & 0 & 0 & 0 & 0 & 0 & 0 & 0 & 0 & 0 \\
\hline 50 & 0 & 0 & 0 & 0 & 0 & 0 & 0 & 0 & 0 & 0 & 8 \\
\hline 60 & 0 & 0 & 0 & 0 & 0 & 0 & 0 & 0 & 0 & 0 & 0 \\
\hline 70 & 0 & 0 & 0 & 0 & 0 & 0 & 0 & 0 & 0 & 0 & 0 \\
\hline 80 & 0 & 0 & 0 & 0 & 0 & 0 & 0 & 2 & 0 & 0 & 0 \\
\hline 90 & 0 & 0 & 0 & 0 & 0 & 0 & 0 & 6 & 0 & 0 & 0 \\
\hline 100 & 0 & 0 & 0 & 0 & 0 & 0 & 9 & 10 & 10 & 10 & 0 \\
\hline \multicolumn{12}{|l|}{ White } \\
\hline 10 & 0 & 0 & 0 & 0 & 0 & 0 & 0 & 0 & 0 & 0 & 0 \\
\hline 20 & 0 & 0 & 0 & 0 & 0 & 0 & 0 & 0 & 0 & 0 & 0 \\
\hline 30 & 0 & 0 & 0 & 0 & 0 & 0 & 0 & 0 & 0 & 0 & 0 \\
\hline 40 & 0 & 0 & 0 & 0 & 0 & 0 & 0 & 0 & 0 & 0 & 0 \\
\hline 50 & 0 & 0 & 0 & 0 & 0 & 0 & 0 & 0 & 0 & 0 & 0 \\
\hline 60 & 0 & 0 & 0 & 0 & 0 & 0 & 0 & 0 & 0 & 0 & 0 \\
\hline 70 & 0 & 0 & 0 & 0 & 0 & 0 & 0 & 0 & 0 & 0 & 0 \\
\hline 80 & 0 & 0 & 0 & 0 & 0 & 0 & 0 & 0 & 0 & 0 & 0 \\
\hline 90 & 0 & 0 & 0 & 0 & 0 & 0 & 0 & 0 & 0 & 0 & 0 \\
\hline 100 & 0 & 0 & 0 & 0 & 0 & 0 & 0 & 0 & 0 & 0 & 2 \\
\hline \multicolumn{12}{|c|}{$\square$ All Misses } \\
\hline
\end{tabular}

The errors as a function of distance and angle of incidence for each of the three targets are shown in Figs. 3.14a to 3.14c and Figs. 3.15a and 3.15b. The scales for the plots in Fig. 3.14 were set to be equal for ease of comparison. The data in Fig. 3.14 are re-plotted in Fig. 3.15 with enlarged scales for the black and white targets. From the plots, it can be seen that, in general, the accuracy decreases as the angle of incidence increases and more specifically, it can be seen that:

1. The silver target yields the largest measurement errors and displays a clear angle of incidence effect on bias that is monotonically decreasing in angle from $80^{\circ}$ down to $30^{\circ}$. It is especially evident, both in terms of magnitude of the bias and clean stratification of biases between angles, for angles of incidences of $50^{\circ}$ and higher.

There might be two possible explanations for the large errors for the silver target. In both of these hypothesized cases, the return signal is affected by external objects such as walls 
around the target. In the first case, the errors may result from splitting of the signal whereby a portion of the signal hits the target with the remaining signal hitting the wall behind the target (see Section 2.6). The size of the target was selected so that the target was larger than the laser beam at the maximum range of the scanner $(150 \mathrm{~m})$. However, as the target is turned, the target area, as seen by the scanner, is reduced and may result in a split signal. The split signal phenomenon does not, however, appear to be contributing to the error in this situation as the errors are only observed for the silver target and not for the white or black targets. This hypothesis is therefore rejected. In the second case, it is assumed that a portion of the signal reflected off the specular target hits an object or wall to the side of the target and is returned to the scanner. In this case, the distance to this object or wall is included in the reported distance measurement - biased high or longer reported distances. This is appears to be the case for the silver target as seen in Fig. $3.14 \mathrm{a}$.

2. The magnitude of the errors for angles of incidence of $\leq 30^{\circ}$ is similar for all targets but the errors for the silver target (highest reflectance) are greater thereafter.

3. For an angle of incidence of $90^{\circ}$, the target as seen by the LADAR is a strip approximately $1 \mathrm{~mm}$ wide. The distance from the target should ostensibly be shorter by half the width of the target ( $0.4 \mathrm{~m}$ wide), i.e., the measured distance should be compared to the true distance minus $0.2 \mathrm{~m}$. This accounts for the consistently negative biases of the $90^{\circ}$ measurements for all three targets.

The averages of the errors $(10 \mathrm{~m}$ to $90 \mathrm{~m})$ for an angle of incidence of $90^{\circ}$ are $-0.312 \mathrm{~m} \pm$ $0.079 \mathrm{~m}^{\dagger}$ for silver, $-0.257 \mathrm{~m} \pm 0.070 \mathrm{~m}$ for white, and $-0.232 \mathrm{~m} \pm 0.053 \mathrm{~m}$ for black. Even after half the width of the target is taken into account, the errors are still large, but it is interesting that the instrument can differentiate an object approximately $1 \mathrm{~mm}$ wide even at $100 \mathrm{~m}$.

4. For the white target, at distances greater than $60 \mathrm{~m}$, the errors appear to be increasing (in the negative direction) almost exponentially (Fig. 3.15a) for the higher angle of incidences.

5. For the black target, there are minor angle of incidence effects. There is some stratification at angles of incidence of $50^{\circ}$ and greater (Fig. 3.15b).

A portion of the error at the higher angles of incidence for all targets may be attributed to the inherent procedure used to determine the range. The LADAR used in these experiments is a time-of-flight instrument. That is, the range is determined by measuring the return time, $t$, of a signal and is approximately equal to $(c \bullet t / 2)$ where $c=$ speed of light and $(t / 2)$ instead of $t$ as $t$ is the total return time. For the LADAR used in these experiments, an "averaging" of the signal return times occurs when no spike or distinct peak in the return signal is detected. For the case when the target is turned away from the scanner, the scanner detects several signal returns (returns from closest point to the farthest point on the angled target) or return times and an average time is recorded. This type of error is instrument specific and cannot be quantified

\footnotetext{
${ }^{\dagger}$ Values following “ $\pm ”$ represent one standard deviation.
} 
unless more information is known about the method used to average the signal return times and further experiments are conducted. The required instrument information is usually not available from the manufacturer for proprietary reasons.

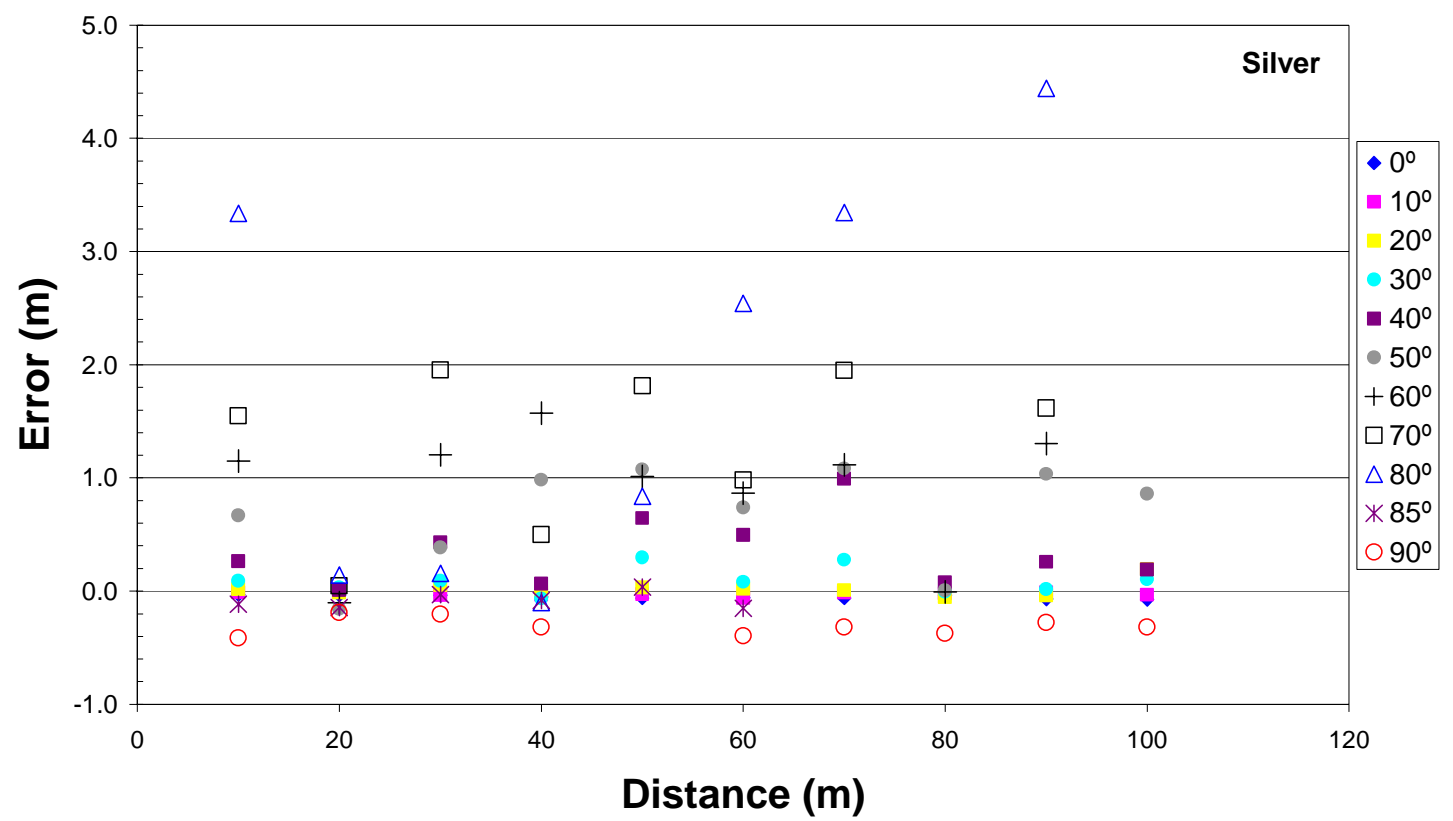

Figure 3.14a. Data Set 6: Silver Target - Error vs. Distance and Angle of Incidence. Note: Errors greater than $5 \mathrm{~m}$ not shown.

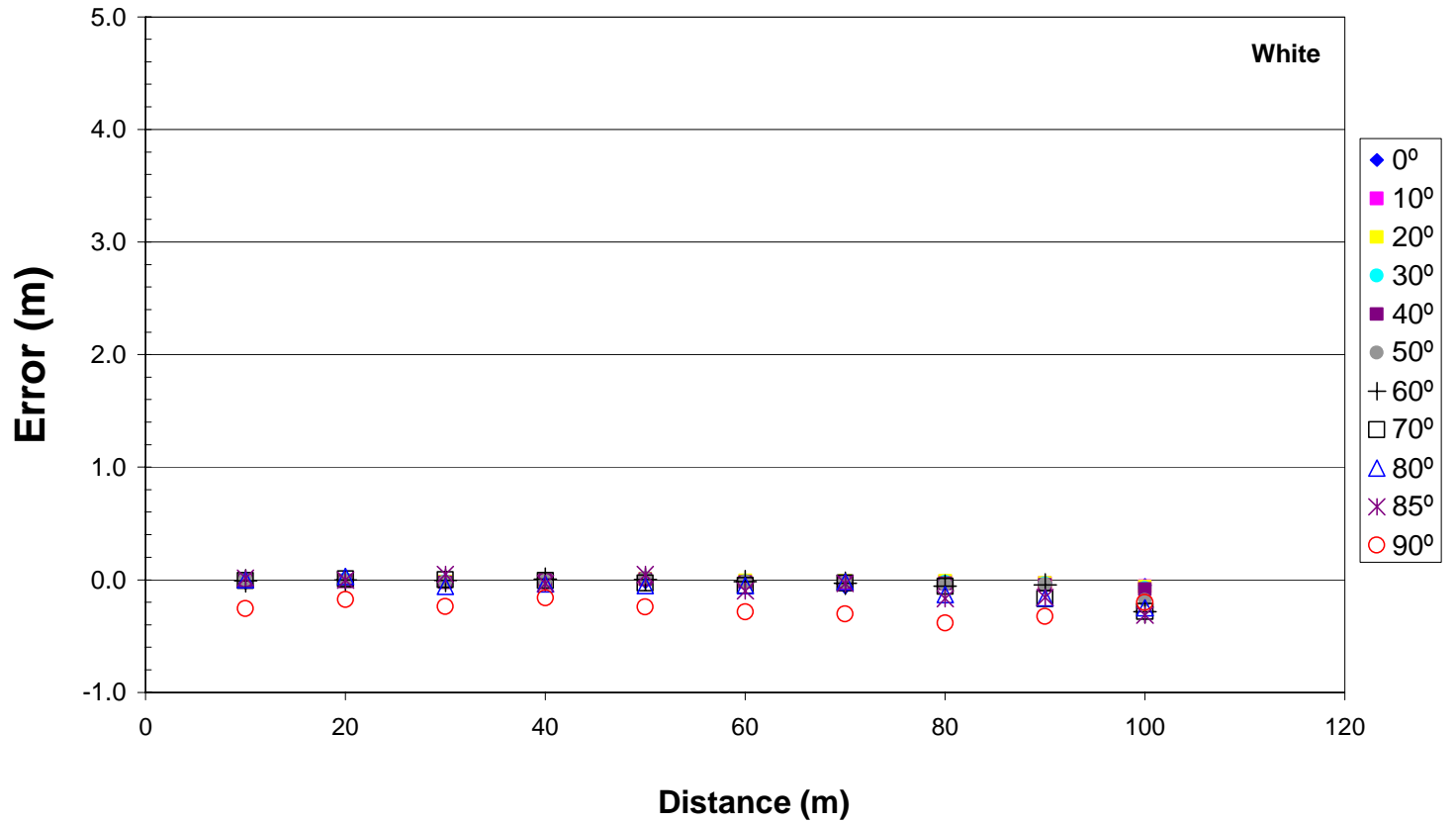

Figure 3.14b. Data Set 6: White Target - Error vs. Distance and Angle of Incidence. 


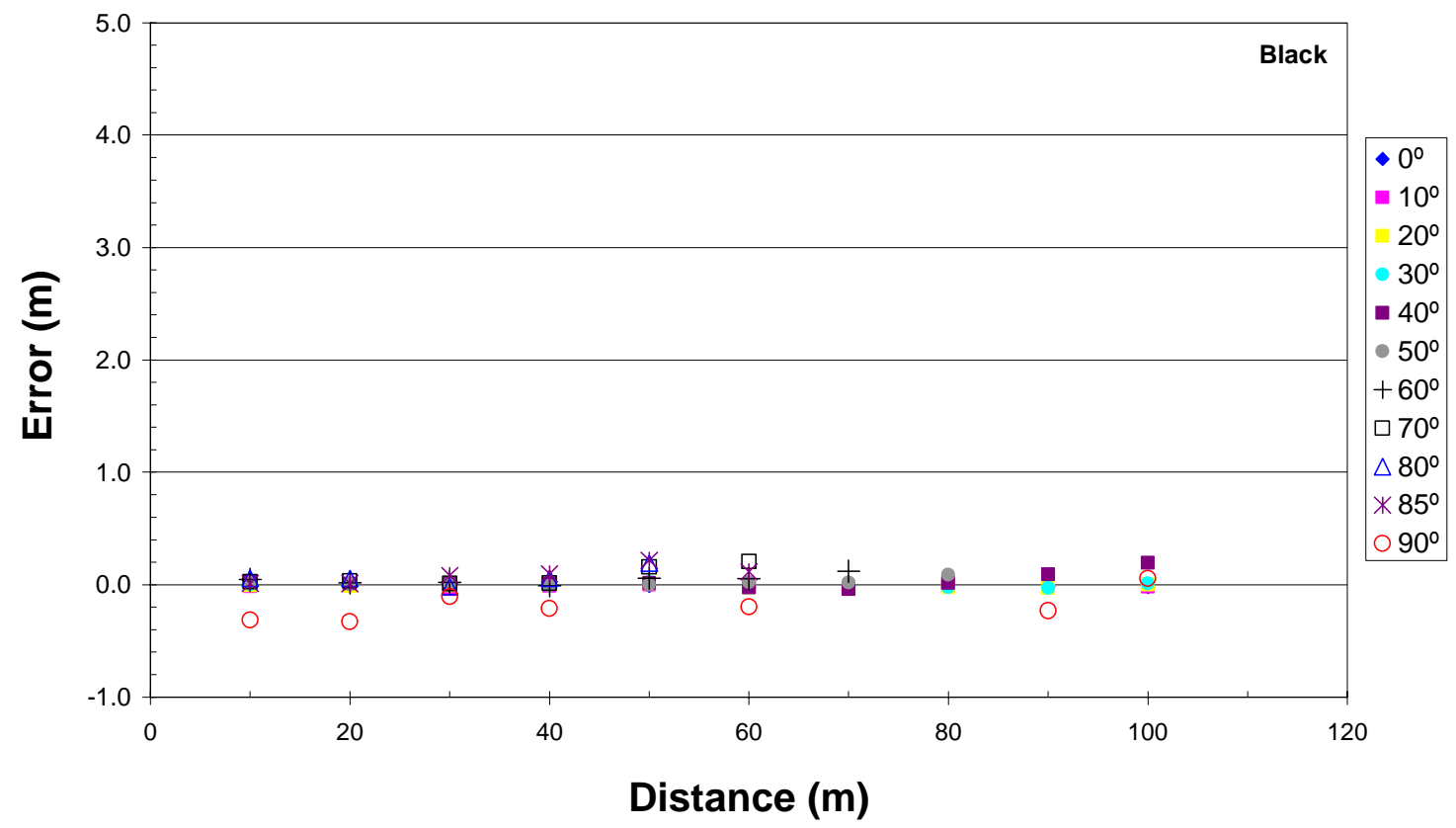

Figure 3.14c. Data Set 6: Black Target - Error vs. Distance and Angle of Incidence.

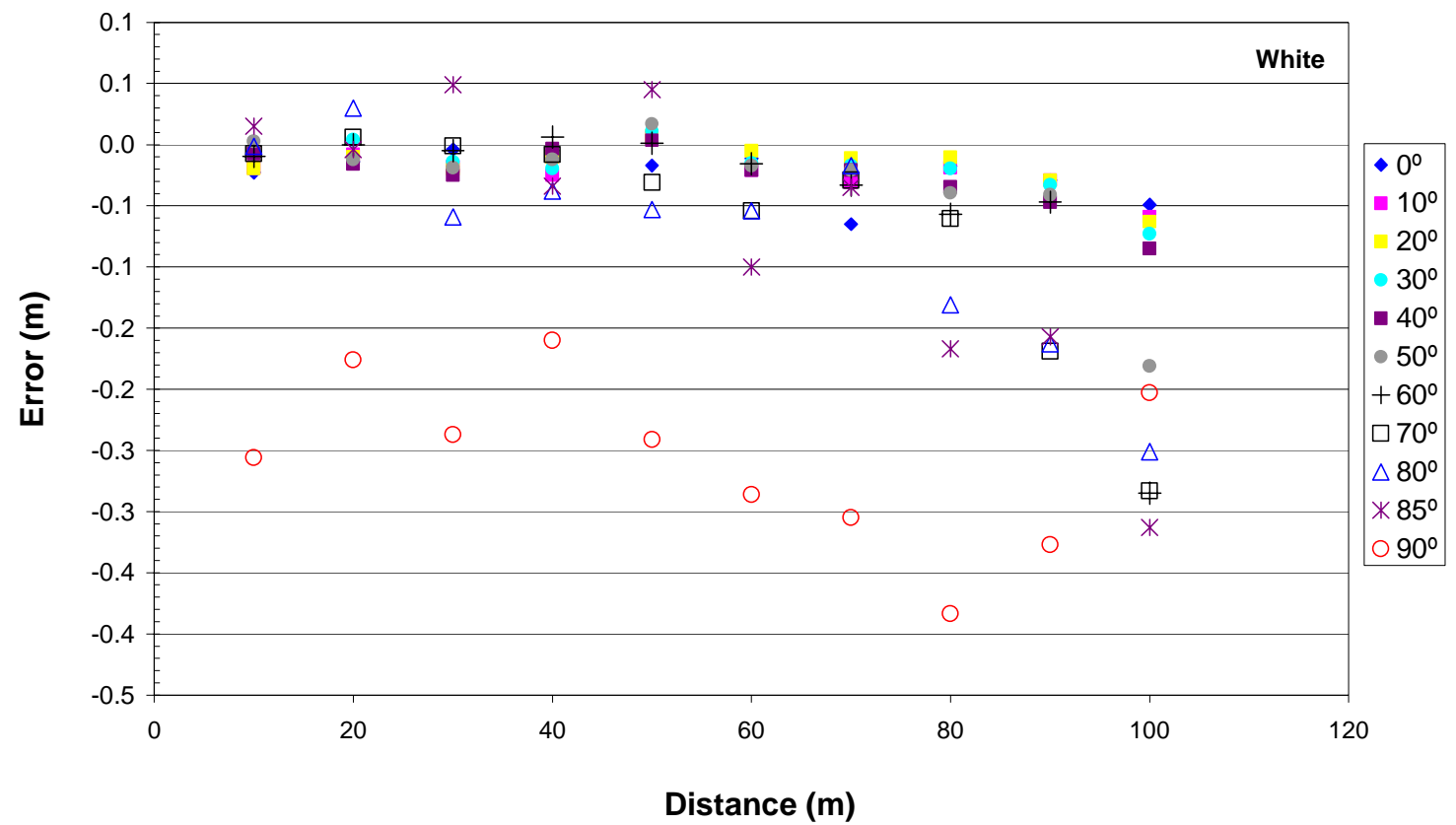

Figure 3.15a. Data Set 6: White Target - Error vs. Distance and Angle of Incidence. (Enlarged scale for Fig. 3.14b) 


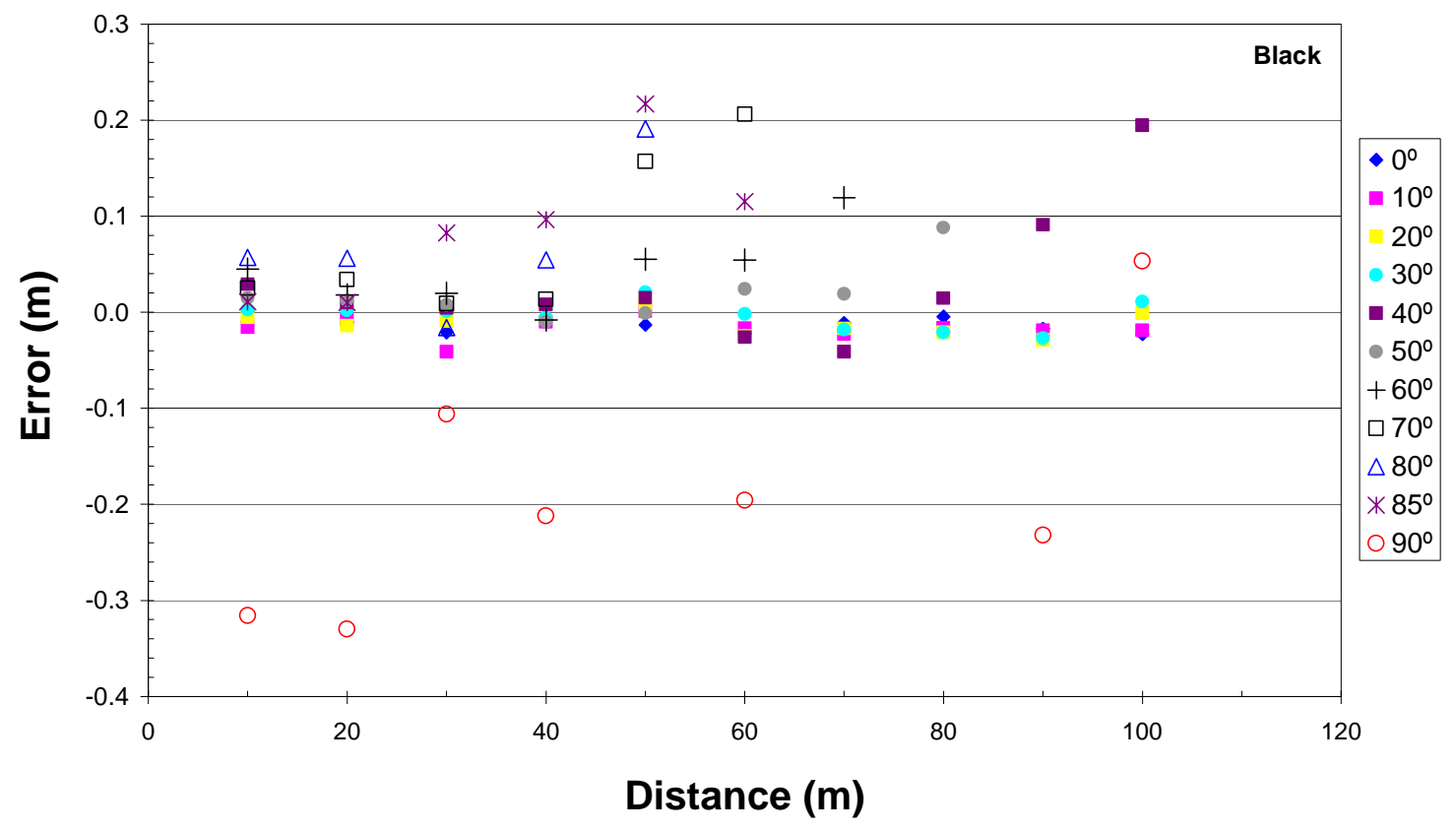

Figure 3.15b. Data Set 6: Black Target - Error vs. Distance and Angle of Incidence.

(Enlarged scale for Fig. 3.14c)

The standard deviations of the errors for Data Set 6 are shown in Figs. 3.16a to 3.16c. It is clear from these plots that the silver target is the noisiest followed by the black target with the white target being the least noisy. Both the silver and black target show a pronounced effect in terms of noise as a function of angle of incidence and distance although the stratification in terms of angle of incidence is not perfectly clean nor is there any immediately obvious monotonicity in terms of magnitude of angle. For distances of $20 \mathrm{~m}$ and greater, the noise seems to increase almost exponentially for high angles of incidence for the silver. 


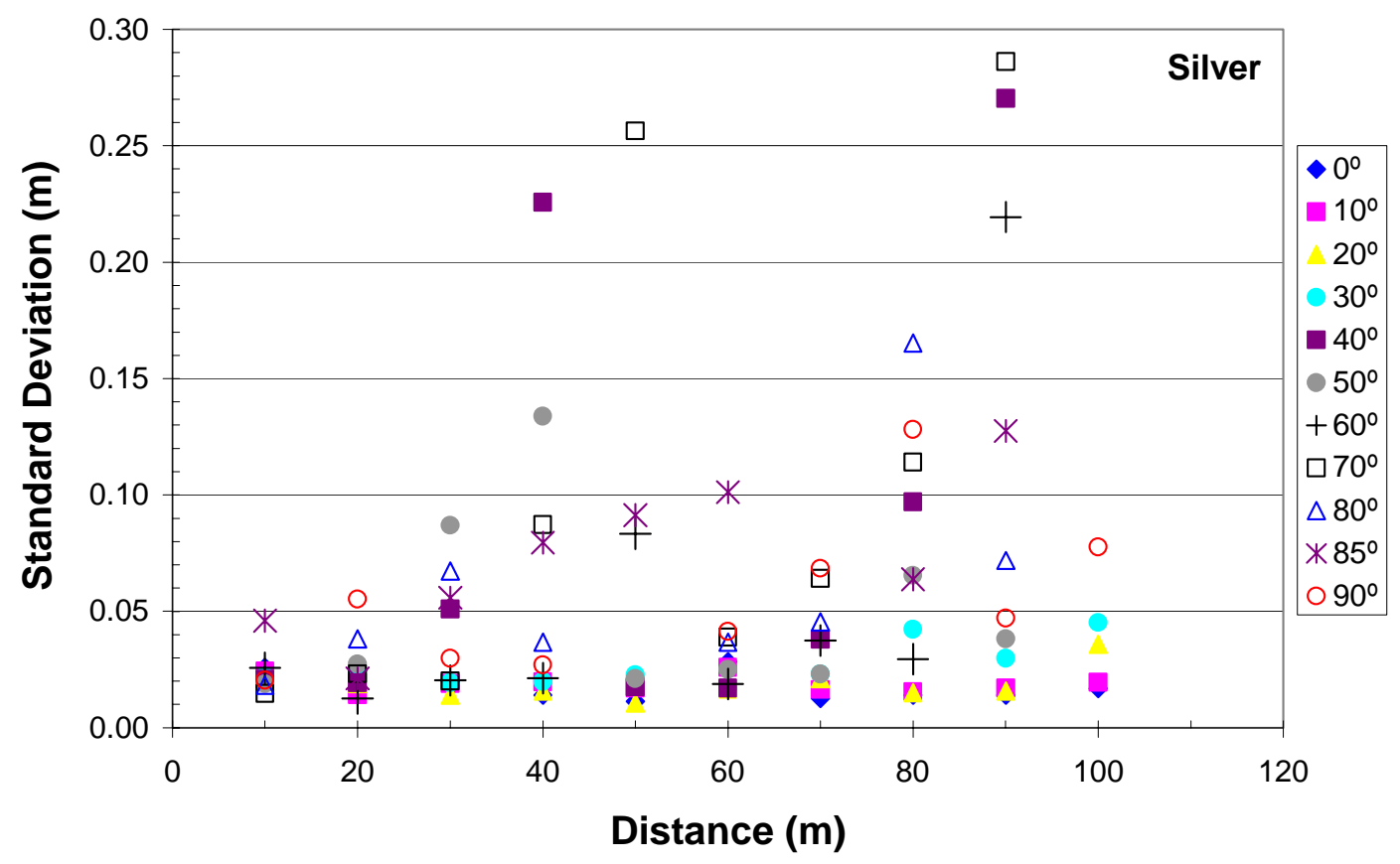

Figure 3.16a. Data Set 6: Standard Deviation of Error for Silver Target.

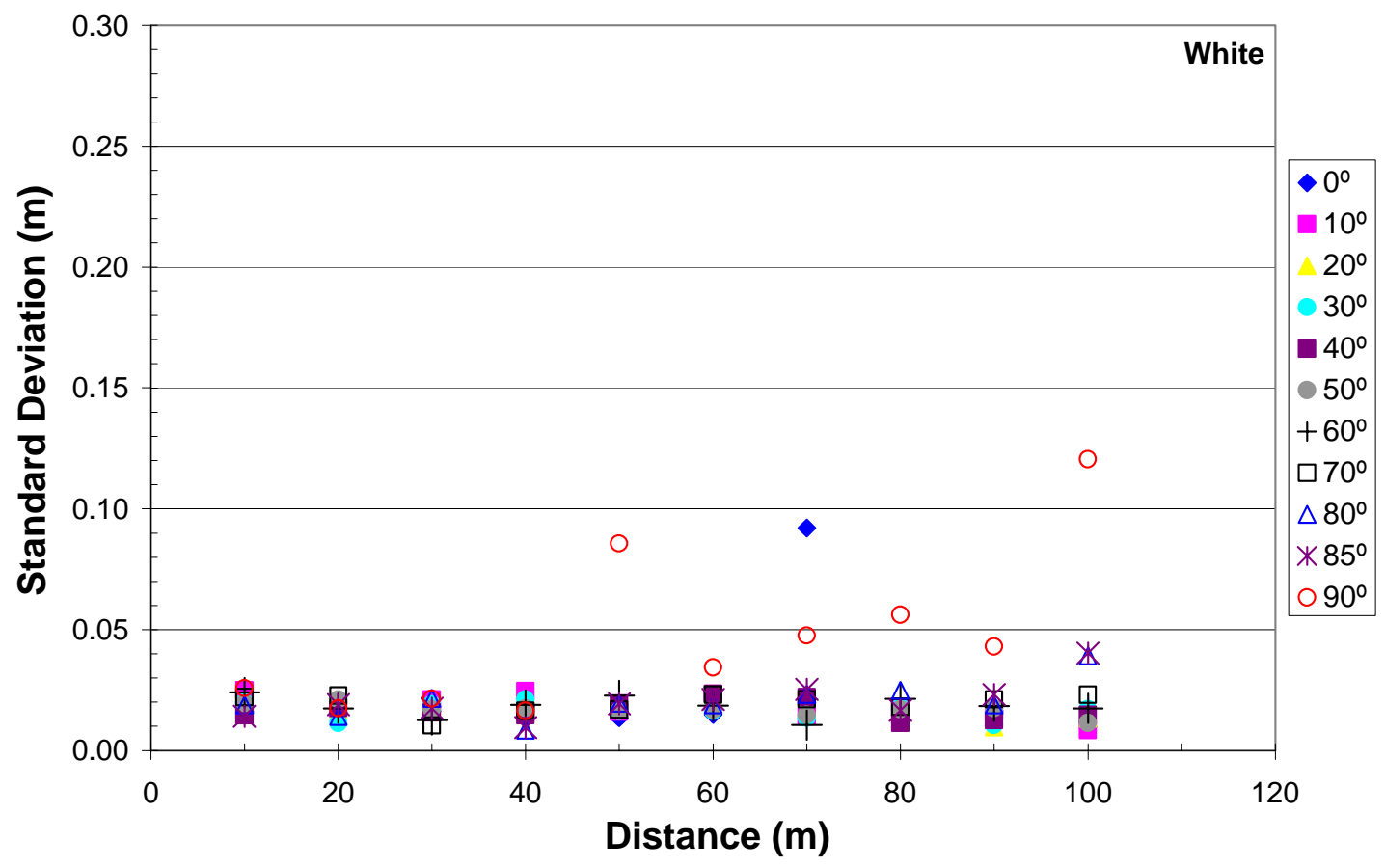

Figure 3.16b. Data Set 6: Standard Deviation of Error for White Target. 


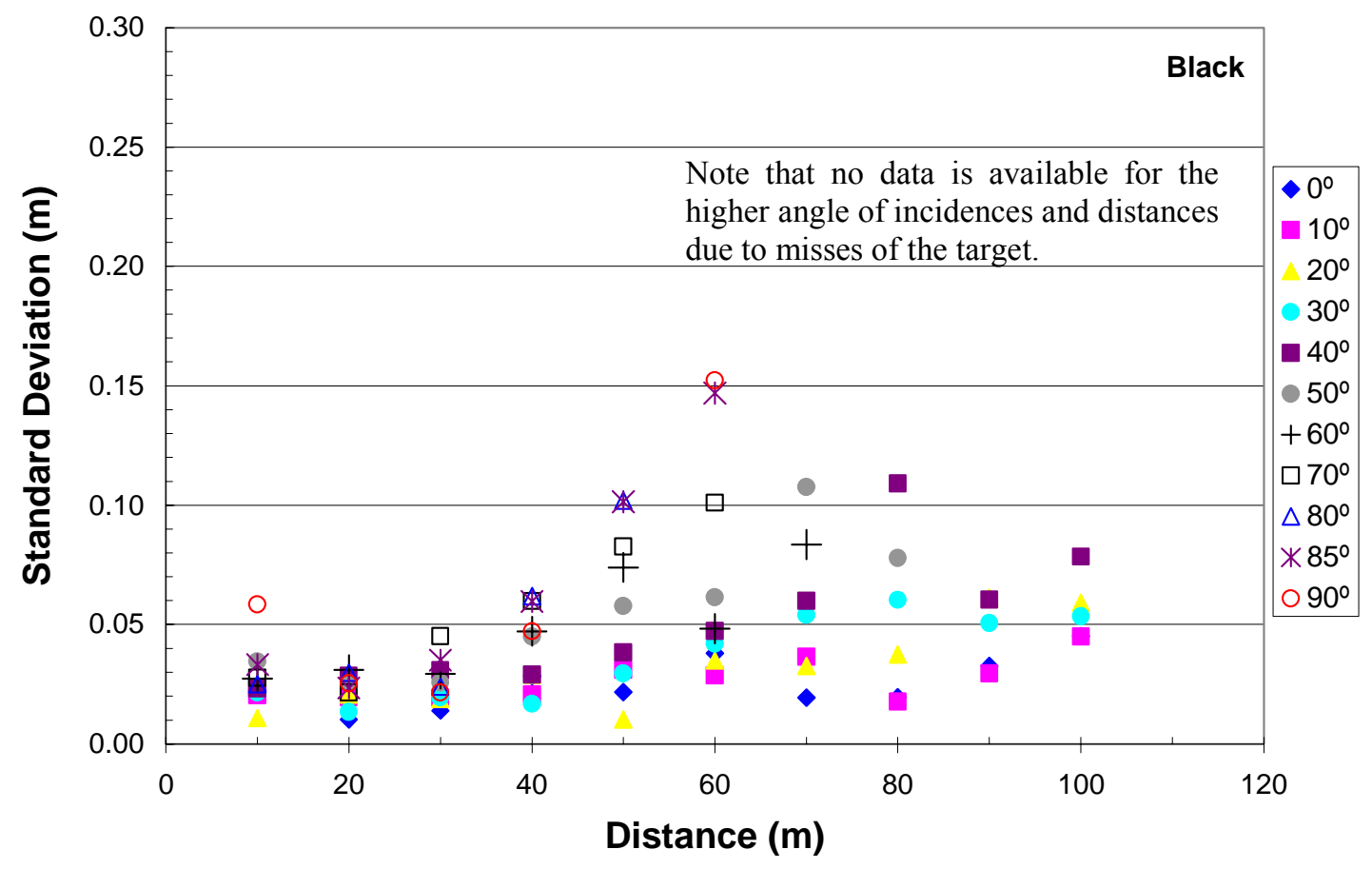

Figure 3.16c. Data Set 6: Standard Deviation of Error for Black Target.

Figures 3.17 and 3.18 combine the data from Figs. 3.15 and 3.16 and show the data for each color separately. In these figures, the values in the legend represent the angular rotation of the target in degrees. 


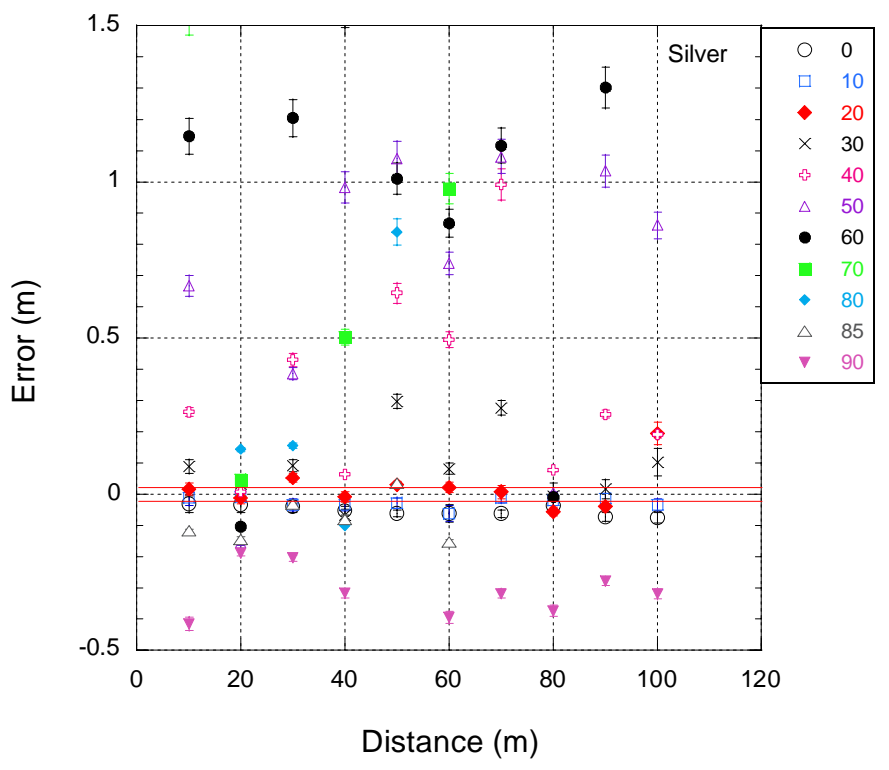

a. Silver

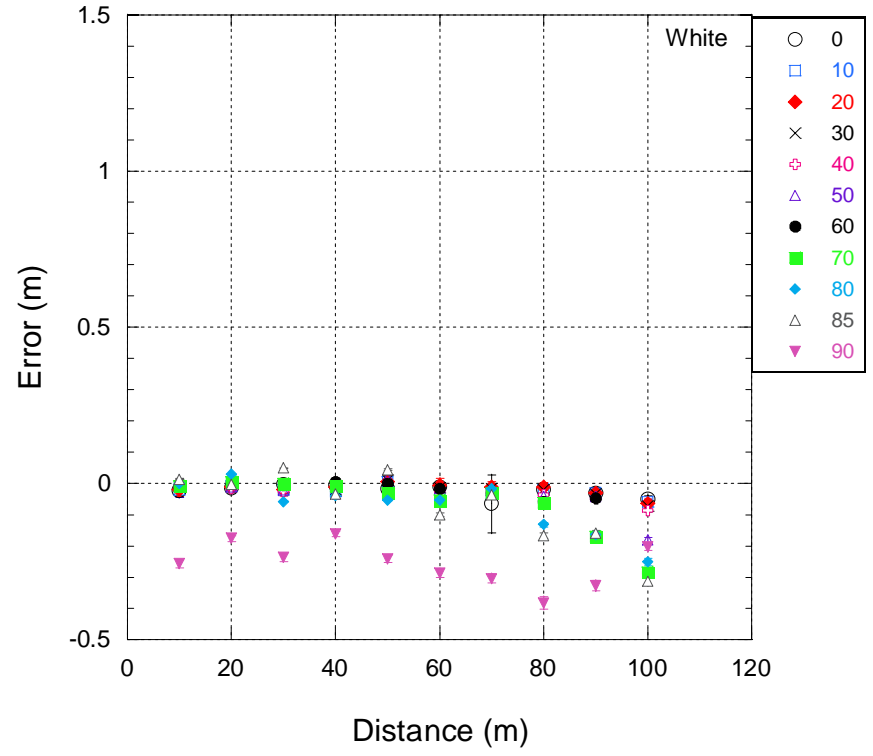

b. White

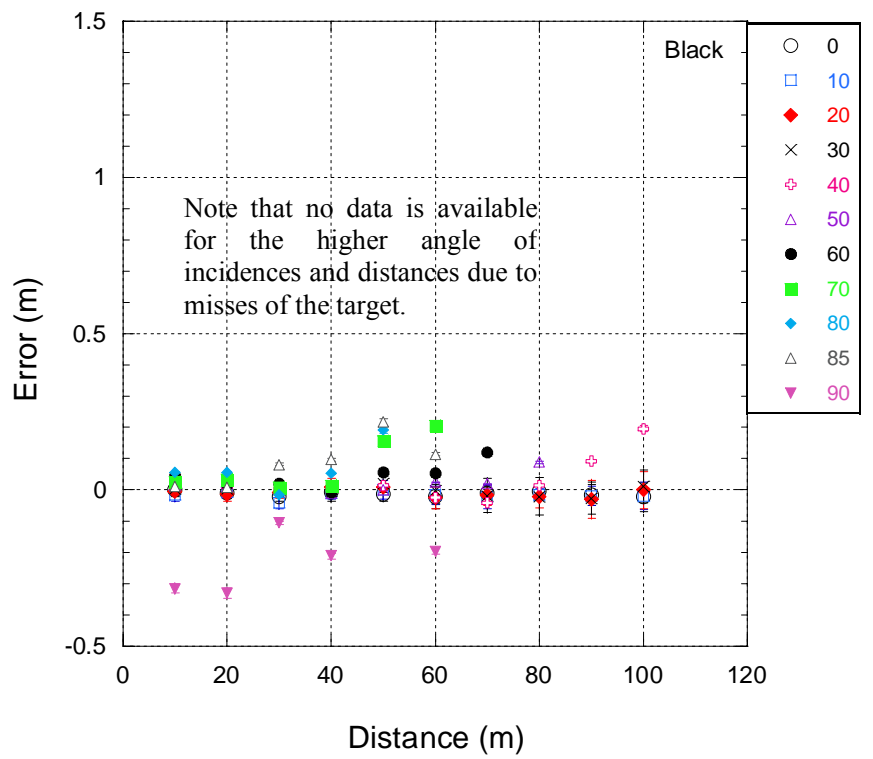

c. Black

Figure 3.17. Data Set 6: Error vs. Distance and Angle of Incidence. Values in legend represent target rotation in degrees. (Error bars $=1 \sigma$ ). 


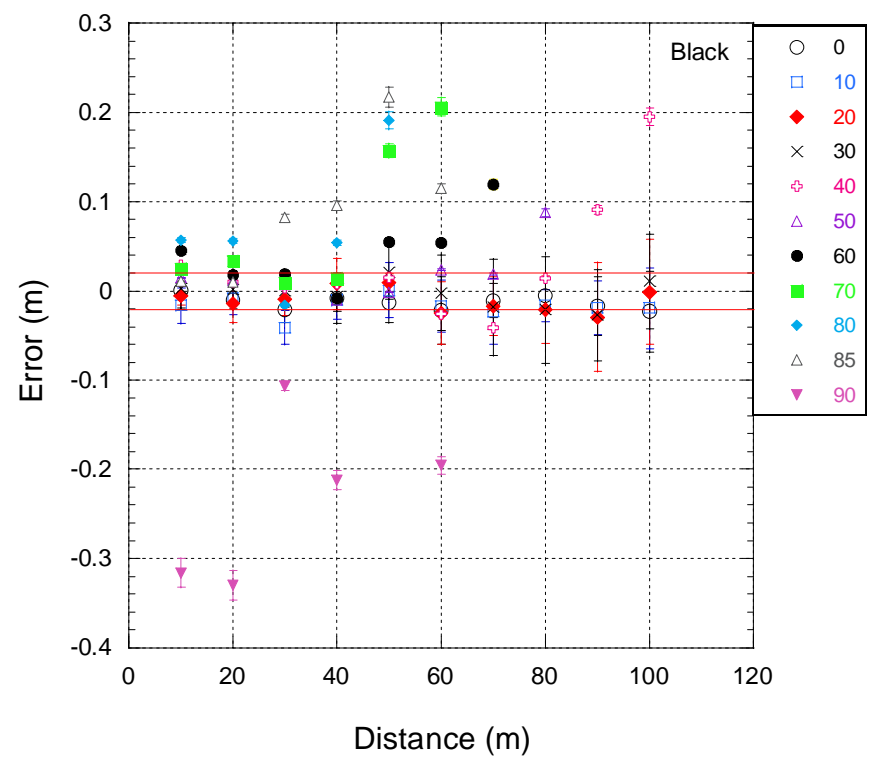

a. Black

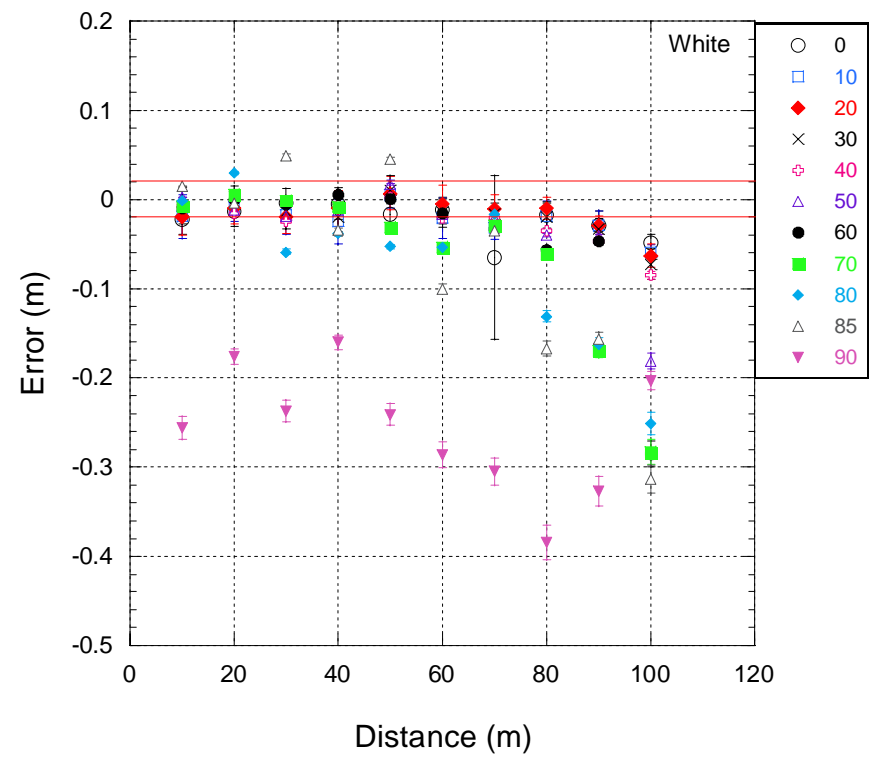

b. White

Figure 3.18. Data Set 6: Error vs. Distance and Angle of Incidence: Enlarged scale as compared with Figs. 3.17b and c. Values in legend represent target rotation in degrees. $($ Error bars $=1 \sigma)$.

\section{$\underline{3.3 .2 \quad \text { Data Sets } 5 \text { and } 6}$}

The data for the LDP target (Data Set 5) for three angles of incidence $\left(0^{\circ}, 45^{\circ}, 60^{\circ}\right)$ are shown in Fig. 3.19a. Other than the observation made in Section 3.1 that high reflectance of the LDP target at $0^{\circ}$ causes significant error for distances less than $40 \mathrm{~m}$, no clear effect in terms of accuracy or precision as a function of angle of incidence, can be observed for the LDP target. The LDP target at $45^{\circ}$ exhibits somewhat degraded accuracy for the mid-distances, $80 \mathrm{~m}$ to $110 \mathrm{~m}$.

Also, shown in Fig. 3.19b for comparison purposes are the data for the silver target (Data Set 6). The silver target, which is less reflective than the LDP target, exhibits greater error than the LDP target for higher angles of incidences. This appears to contradict the observation made earlier that errors for more reflective targets arise from the presence of objects around the target. A possible explanation for this apparent contradiction may be because the LDP material consists of many small individual highly reflective prisms (Fig. 2.2 b). The material is manufactured in this manner (maximum visibility) so that light is reflected back to an observer even if the target is rotated away from the observer. 


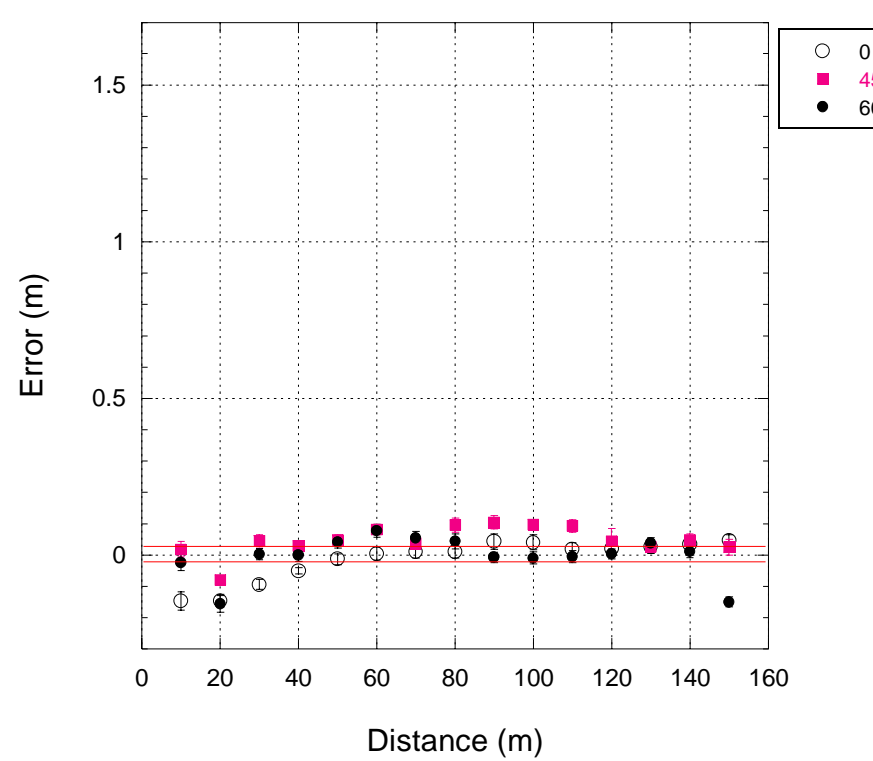

a. LDP Target

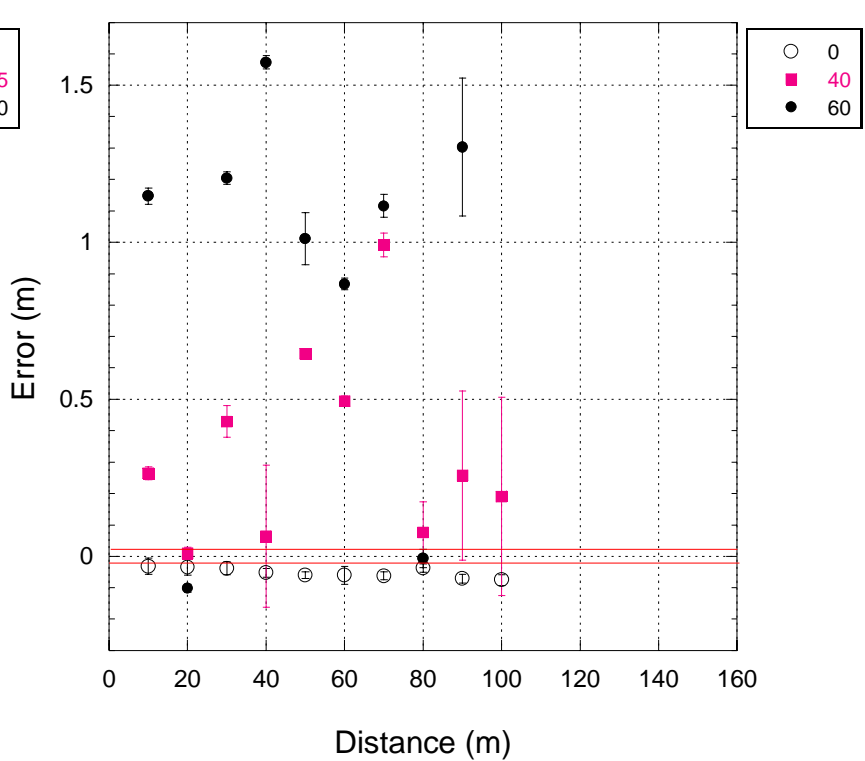

b. Silver Target

Figure 3.19. Error vs. Distance and Angle of Incidence for LDP Targets (Data Set 5) and Silver Target (Data Set 6). Values in legend represent target rotation in degrees. (Error bars $=1 \sigma)$.

Summary of findings for angle of incidence: As expected, the accuracy and precision decrease as the angle of incidence increases, with the silver target (most reflective) yielding the greatest errors and being the least precise. There is no clear pattern for the effect of reflectance on precision. However, reflectance is seen to have an effect on the number of misses of a target with a lower reflectance target sustaining more misses at higher angles of incidence and at larger distances.

\subsection{Beam Divergence}

A useful byproduct of the angle of incidence experiment (Data Set 6) is the data of the laser beam size as a function of distance from the target. This information can be used to determine the beam spread function ${ }^{*}$ and the divergence of the laser beam. A laser producing a smaller output beam diameter with minimum divergence will yield more accurate distance measurements as the reported distance is averaged over the illuminated area.

The laser in the scanner is comprised of three laser diodes. The beam size as it exits the LADAR is $42 \mathrm{~mm}$ (high) x $25 \mathrm{~mm}$ (wide) as stated by the manufacturer. The results of the experiment show that the projections of the laser are one bright rectangular region for distances less than

\footnotetext{
* The beam spread function is necessary for the deconvolution of the LADAR intensity image which allows for the identification of the image or "reading" of the bar codes. This topic is the subject of another NIST internal report (in preparation).
} 
$10 \mathrm{~m}$. For distances greater than $10 \mathrm{~m}$, three bright vertical bands with dark regions in between are visible.

The visual procedure to determine beam sizes is as follows (recall that the outlines of the laser beam were made by at least two observers using different color markers):

1. Make two copies of original outlines (or as many copies as there are observers).

2. Draw first line to best fit Observer's 1 markings - Line 1 (Fig. 3.20). Note: Line 1 was arbitrarily chosen - Lines 2,3 , or 4 could easily have been chosen as the first line to be drawn.

3. Draw a second line $90^{\circ}$ to Line 1 that best fit markings - Line 2 .

4. $\quad$ Draw a third line $90^{\circ}$ to Line 2 that best fit markings - Line 3 .

5. Draw a fourth line $90^{\circ}$ to Line 3 that best fit markings - Line 4 .

6. Draw lines 5-8 perpendicular to Line 2 or Line 4.

7. Measure lengths of lines.

8. Repeat Steps 2 to 7 for Observer 2's outlines.

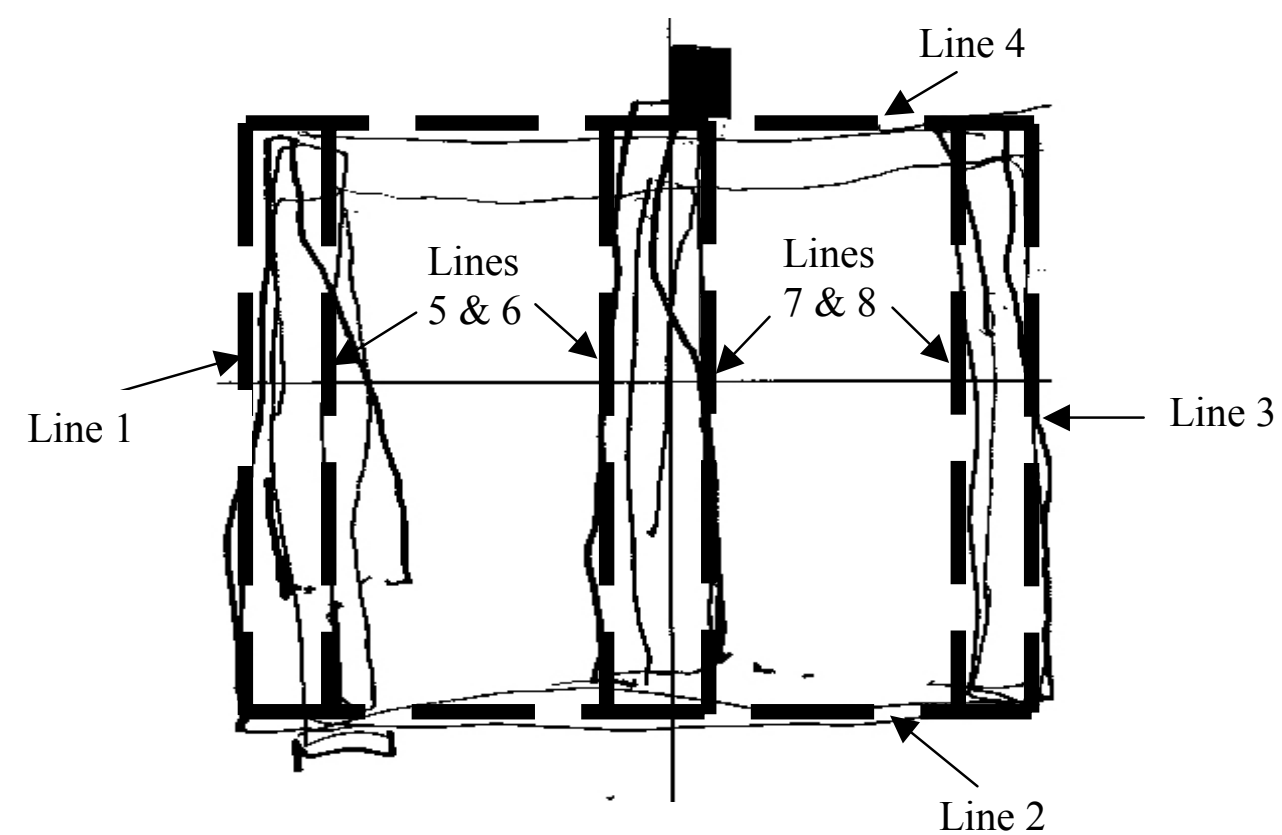

(a) Observer 1 (Drawing has been reduced and is not shown to scale).

Figure 3.20. Outline of Laser Beam at $70 \mathrm{~m}$. 


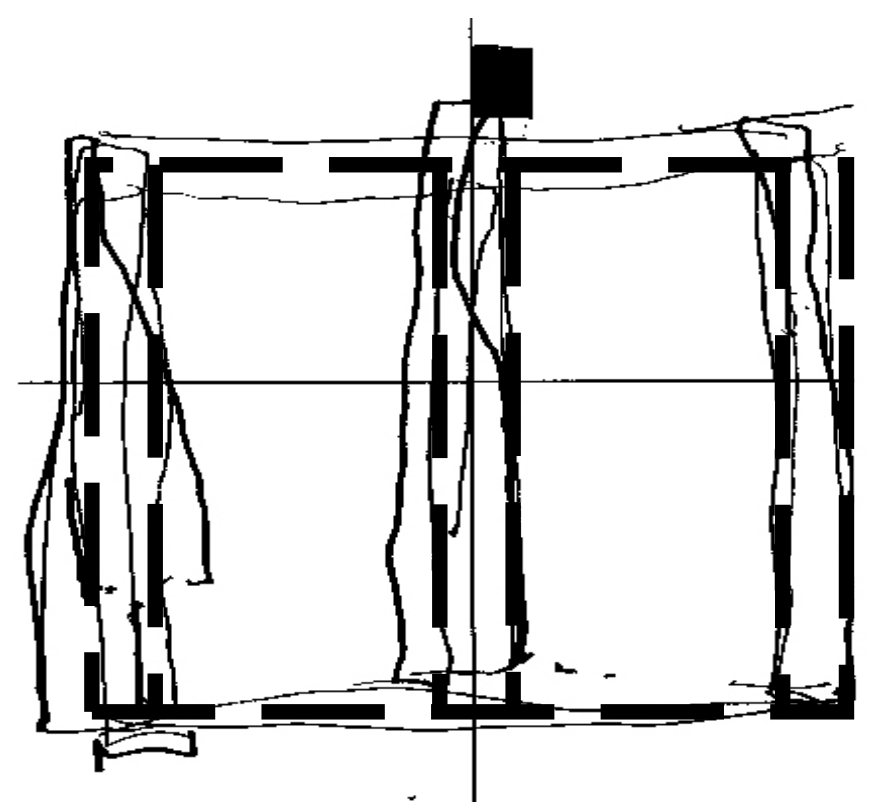

(b) Observer 2 (Drawing has been reduced and is not shown to scale).

Continue Figure 3.20. Outline of Laser Beam at $70 \mathrm{~m}$.

The beam dimensions as obtained from the procedure described above are given in Table 3.6.

Table 3.6. Data Set 6: Laser Beam Size.

\begin{tabular}{|c|c|c|c|c|c|c|c|}
\hline $\begin{array}{c}\text { Distance } \\
(\mathbf{m})\end{array}$ & $\begin{array}{c}\mathbf{L}^{\dagger} \\
(\mathbf{m m})\end{array}$ & $\begin{array}{c}\mathbf{W}^{\dagger} \\
(\mathbf{m m})\end{array}$ & $\begin{array}{c}\mathbf{a}^{\dagger} \\
(\mathbf{m m})\end{array}$ & $\begin{array}{c}\mathbf{b}^{\dagger} \\
(\mathbf{m m})\end{array}$ & $\begin{array}{c}\mathbf{c}^{\dagger} \\
(\mathbf{m m})\end{array}$ & $\begin{array}{c}\mathbf{d}^{\dagger} \\
(\mathbf{m m})\end{array}$ & $\begin{array}{c}\mathbf{e}^{\dagger} \\
(\mathbf{m m})\end{array}$ \\
\hline $2^{\S}$ & 17 & 41 & na & na & na & na & na \\
\hline $2^{\S}$ & 15 & 45.5 & na & na & na & na & na \\
\hline $2^{\S}$ & 15 & 47 & na & na & na & na & na \\
\hline $2^{\S}$ & 17 & 40 & na & na & na & na & na \\
\hline $2^{\S}$ & 14 & 41 & na & na & na & na & na \\
\hline $5^{\S}$ & 19 & 42 & na & na & na & na & na \\
\hline $5^{\S}$ & 25 & 46 & na & na & na & na & na \\
\hline $5^{\S}$ & 18 & 36 & na & na & na & na & na \\
\hline $5^{\S}$ & 19 & 41 & na & na & na & na & na \\
\hline $10^{\ddagger}$ & 31.5 & 49 & 15 & 2 & 10.5 & 1.5 & 20 \\
\hline 10 & 27 & 51 & na & na & na & na & na \\
\hline 10 & 28 & 48 & na & na & na & na & na \\
\hline 10 & 22 & 46 & na & na & na & na & na \\
\hline 10 & 29 & 58 & na & na & na & na & na \\
\hline 10 & 24 & 53 & na & na & na & na & na \\
\hline 10 & 31 & 62 & na & na & na & na & na \\
\hline $20^{\ddagger}$ & 60 & 68 & 19.5 & 8 & 16 & 10 & 14 \\
\hline 20 & 53.5 & 68 & 9.5 & 16.5 & 14 & 14 & 14 \\
\hline 20 & 55 & 56 & 9 & 15 & 14 & 11 & 8 \\
\hline 30 & 86 & 79 & 7 & 21.5 & 11 & 29 & 10 \\
\hline 30 & 79 & 87 & 9.5 & 24.5 & 15 & 34.5 & 3.5 \\
\hline $30^{\ddagger}$ & 74.5 & 86 & 16 & 18 & 16.5 & 23.5 & 12 \\
\hline $39.5^{\ddagger}$ & 117.5 & 116 & 11.5 & 37.5 & 14.5 & 37 & 15.5 \\
\hline
\end{tabular}




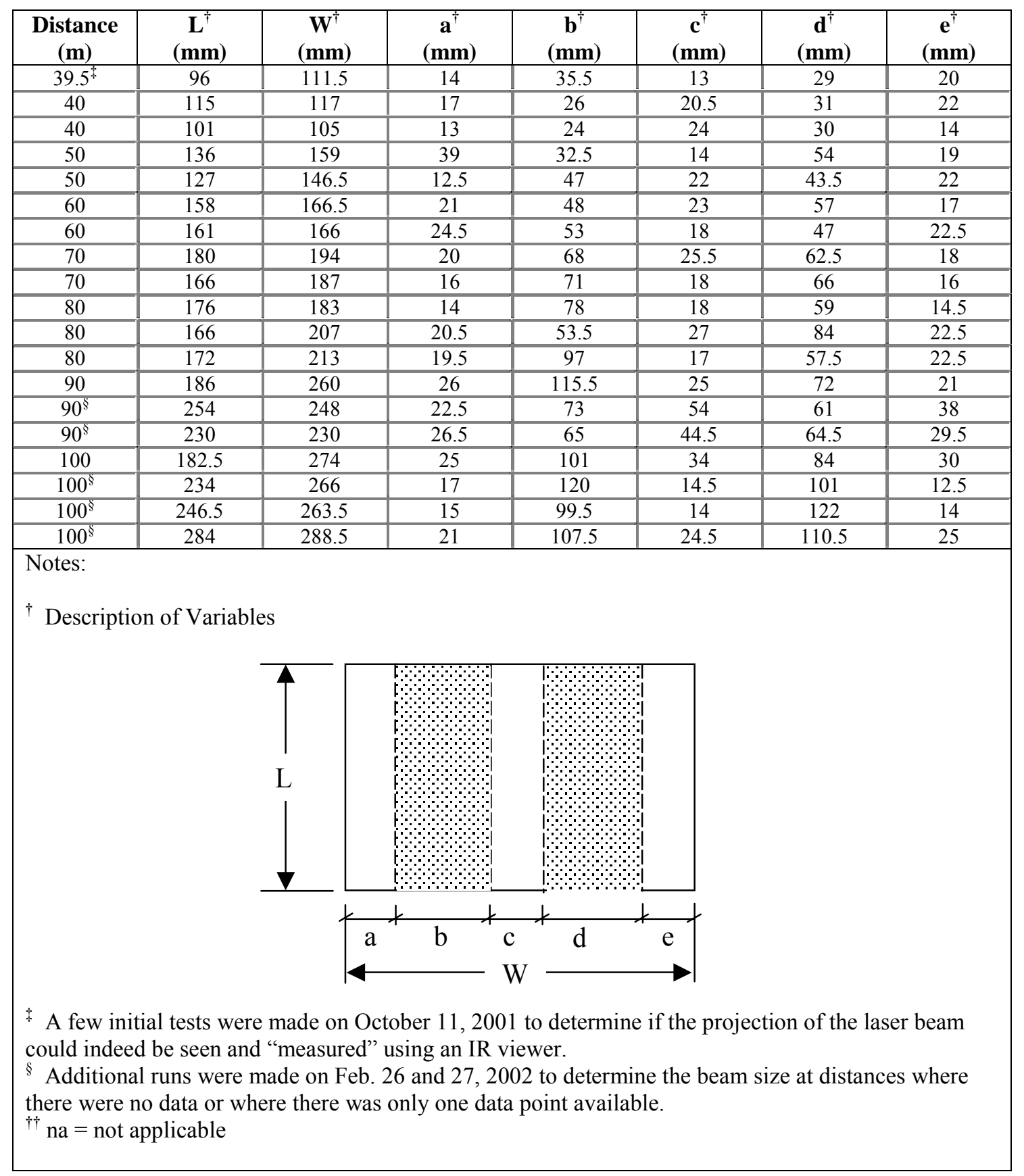

The lengths and widths of the beam projection are plotted as a function of the distance in Fig. 3.21. Despite the highly subjective manner in which the beam dimensions were obtained, a clear trend for the beam length and width appears visible in Fig. 3.21. Quadratic model least squares regression parameters for the width and length of the beam are: 


$$
\begin{array}{ll}
\text { width }=0.0071 x^{2}+1.6637 x+34.815 & R^{2}=0.9874 \\
\text { length }=-0.0025 x^{2}+2.528 x+6.9588 & R^{2}=0.9606 \\
\text { for } 5 \leq \mathrm{x} \leq 100 &
\end{array}
$$

where

$$
x \text { is the distance in meters }
$$

and beam width, length are in millimeters

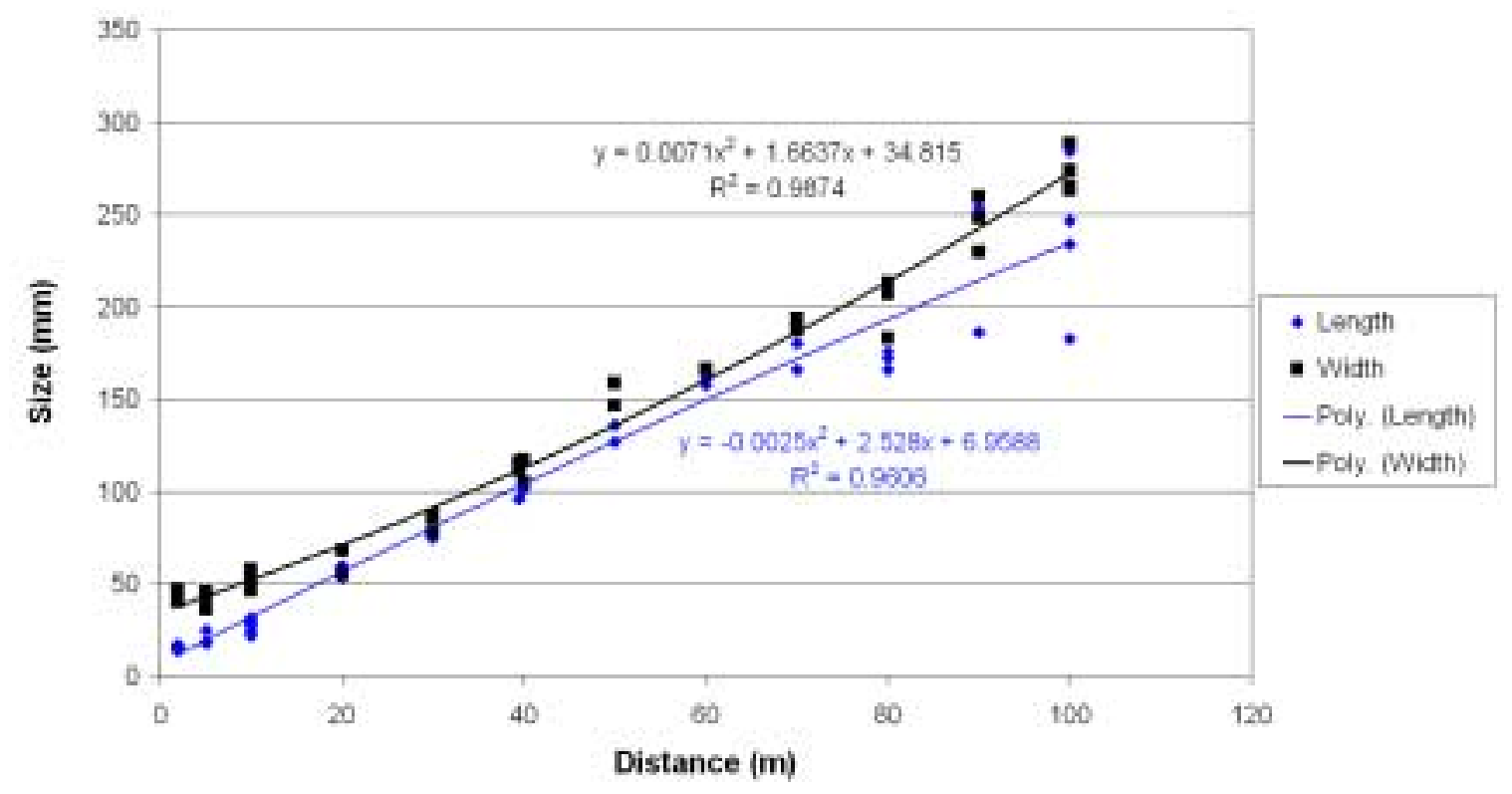

Figure 3.21. Data Set 6: Regression Fits for Laser Beam Length and Width.

Based on the measured beam dimensions, divergences of the beam in the width (horizontal) and length (vertical) directions were calculated. Since there were no measurements of the beam size as it exits the instrument, the beam size at $2 \mathrm{~m}$ was taken as the reference or initial beam size for calculating the divergence. The divergence was calculated using the formulas $(\tan \gamma \approx \gamma$ for $\gamma<<$ 1): 


$$
\begin{aligned}
& \gamma_{\text {vertical }}(x)=\left(\frac{L(x)-L(2)}{(D(x)-D(2)) * 1000}\right) * 1000=\left(\frac{L(x)-15.55}{(D(x)-2)^{*} 1000}\right) * 1000 \\
& \gamma_{\text {horizontal }}(x)=\left(\frac{W(x)-W(2)}{(D(x)-D(2)) * 1000}\right) * 1000=\left(\frac{W(x)-43}{\left.(D(x)-2)^{*} 1000\right)}\right) * 1000
\end{aligned}
$$

where

$\gamma_{\text {vertical }}(x), \gamma_{\text {horizontal }}(x)=$ divergence at distance $x$ in milliradians

$L(x)=$ Average length, in millimeters, of beam at distance $x$

$W(x)=$ Average width, in millimeters, of beam at distance $x$

$D(x)=$ Distance in meters

The beam divergences as a function of distance are shown in Fig. 3.22.

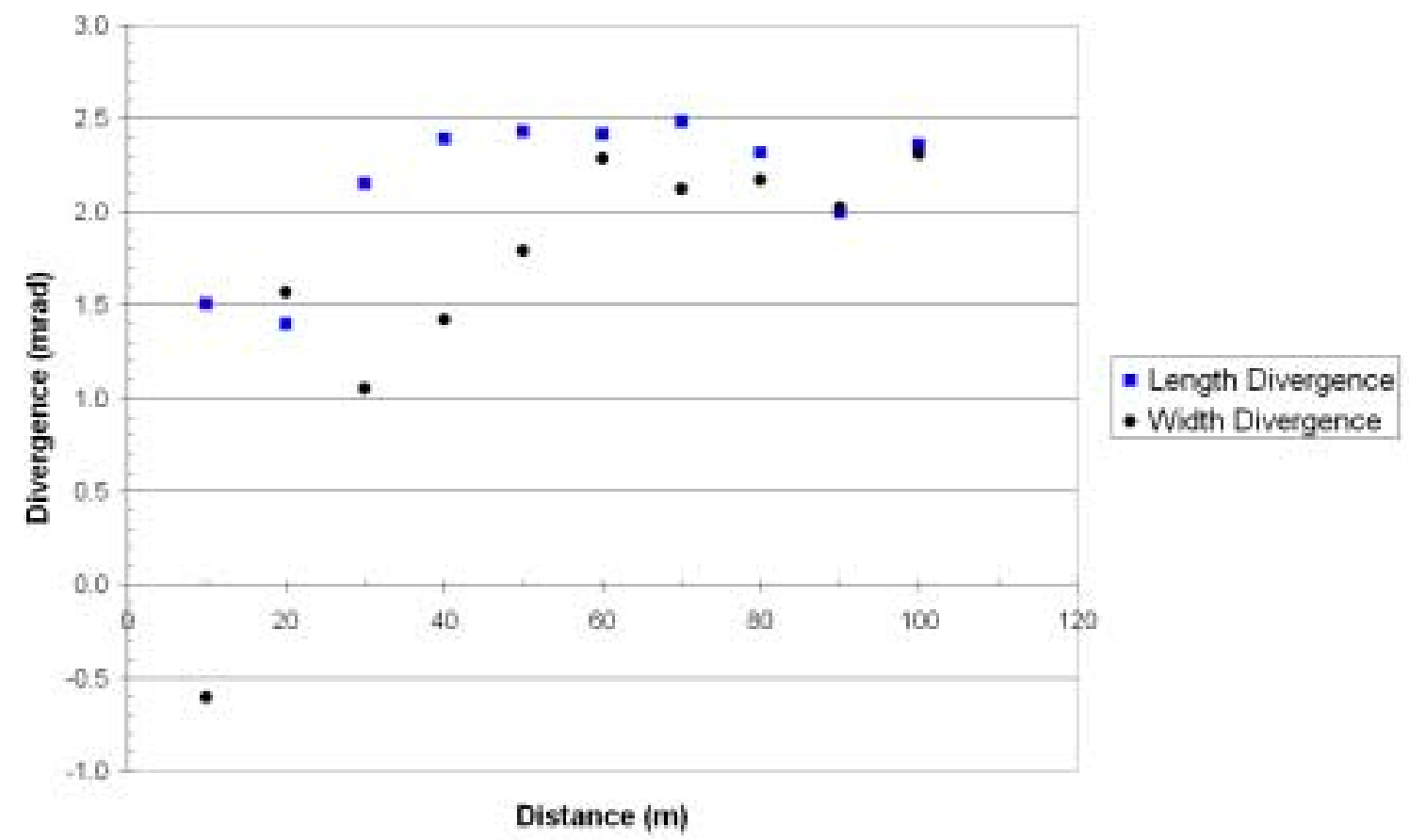

Figure 3.22. Beam Divergence.

The average vertical beam divergence is $2.14 \mathrm{mrad}(\sigma=0.39 \mathrm{mrad})$ and the average horizontal beam divergence, excluding the outlier (negative divergence) is $1.86 \mathrm{mrad}(\sigma=0.44 \mathrm{mrad})$. The average beam divergence, average of horizontal and vertical divergences, is $2.01 \mathrm{mrad}(\sigma=$ $0.43 \mathrm{mrad}$ ) - compared with the manufacturer's specified divergence of $3 \mathrm{mrad}$. The lower experimental value could be a result of the inability of the unaided human eye to detect the faint edges of the laser beam projection. 
Plots of the bandwidths of the bright and dark bands are shown in Figs. 3.23 and 3.24. In Fig. 3.23, the bandwidths for the each individual band are plotted and trends are clearly visible for both the bright and dark bands. Linear regression parameters for individual bandwidths are given in Table 3.7.

Table 3.7. Coefficients for Linear Regression for Individual Bandwidths.

\begin{tabular}{|c|c|c|c|c|}
\hline Description & $\begin{array}{c}\text { Bandwidth }^{\dagger} \\
(\mathbf{m m})\end{array}$ & Slope $(\mathbf{M})^{\dagger}$ & Intercept $(\mathbf{B})^{\dagger}$ & $\mathbf{R}^{2 \vdots}$ \\
\hline \multicolumn{5}{|l|}{ Bright Bands } \\
\hline Left & $a^{\S}$ & 0.1123 & 11.198 & 0.2283 \\
\hline Middle & $c^{\S}$ & 0.1842 & 9.8815 & 0.2938 \\
\hline Right & $\mathrm{e}^{\S}$ & 0.1315 & 10.643 & 0.2864 \\
\hline \multicolumn{5}{|l|}{ Dark Bands } \\
\hline Left & $\mathrm{b}^{\S}$ & 1.1426 & -13.251 & 0.8954 \\
\hline Right & $d^{\S}$ & 0.9815 & -6.0425 & 0.8702 \\
\hline \multicolumn{5}{|c|}{$\begin{array}{l}\text { Notes: } \\
\begin{array}{l}\text { Bandwidth }=\mathrm{Mx}+\mathrm{B} \text { where } \mathrm{x}=\text { distance in meters; } 5<\mathrm{x}<100 \\
\text { Correlation coefficient squared } \\
\$ \text { Corresponds to bands shown in Notes section of Table 3.6. }\end{array}\end{array}$} \\
\hline
\end{tabular}

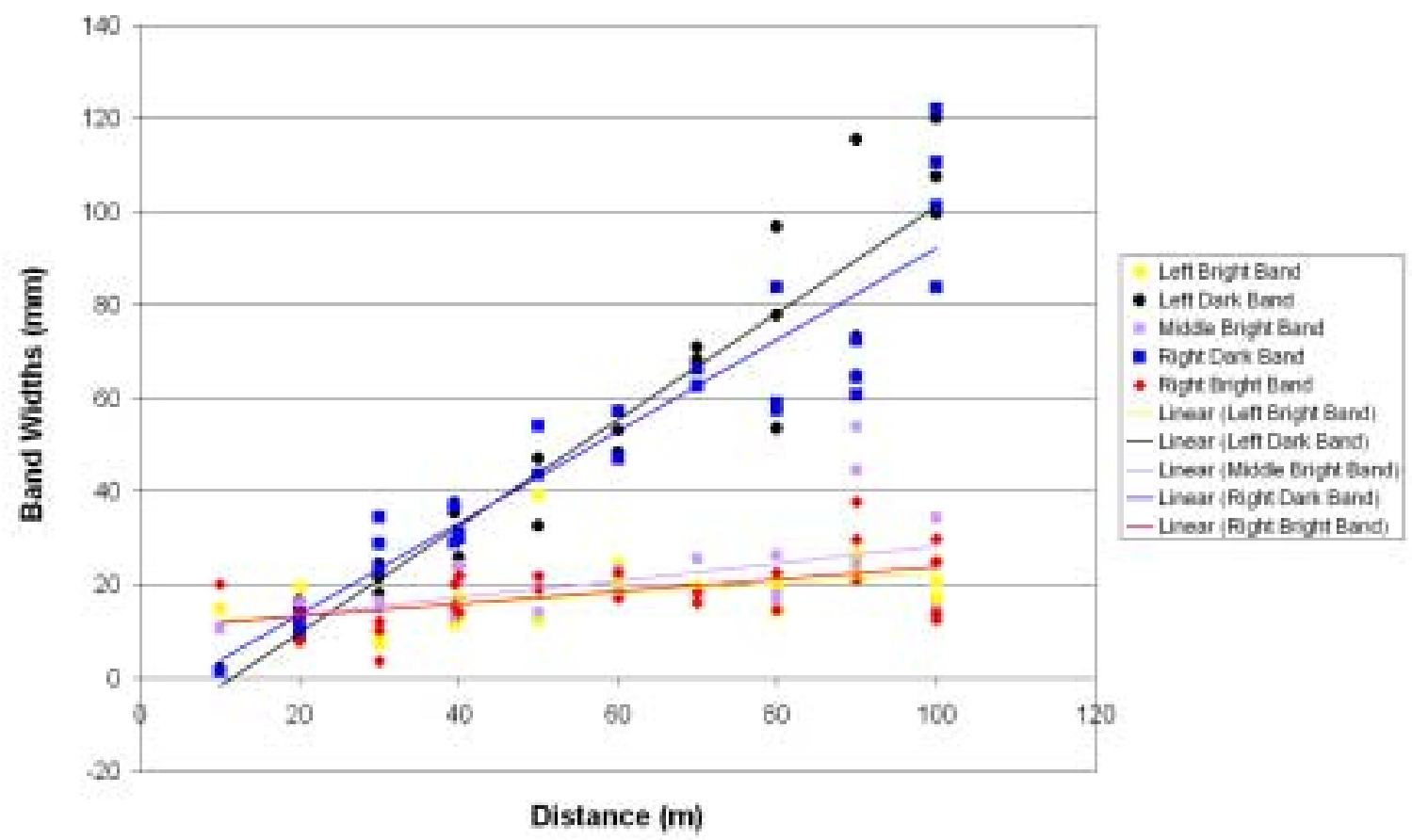

Figure 3.23. Individual Band Widths vs Distance. 
Fig. 3.24 is a plot of the same data as shown in Fig. 3.23. With the data for the three bright bands combined and plotted as 'bright band' and the data for the two dark bands combined and plotted as 'dark band'. The regression parameters for the bright and dark bandwidth fits are:

$$
\begin{array}{ll}
\text { Dark bandwidth }=1.062 x-9.6468 & R^{2}=0.8784 \\
\text { Bright bandwidth }=0.143 x+10.574 & R^{2}=0.2578
\end{array}
$$

where

$\mathrm{x}=$ distance in meters; $10<x<100$

bandwidth in millimeters

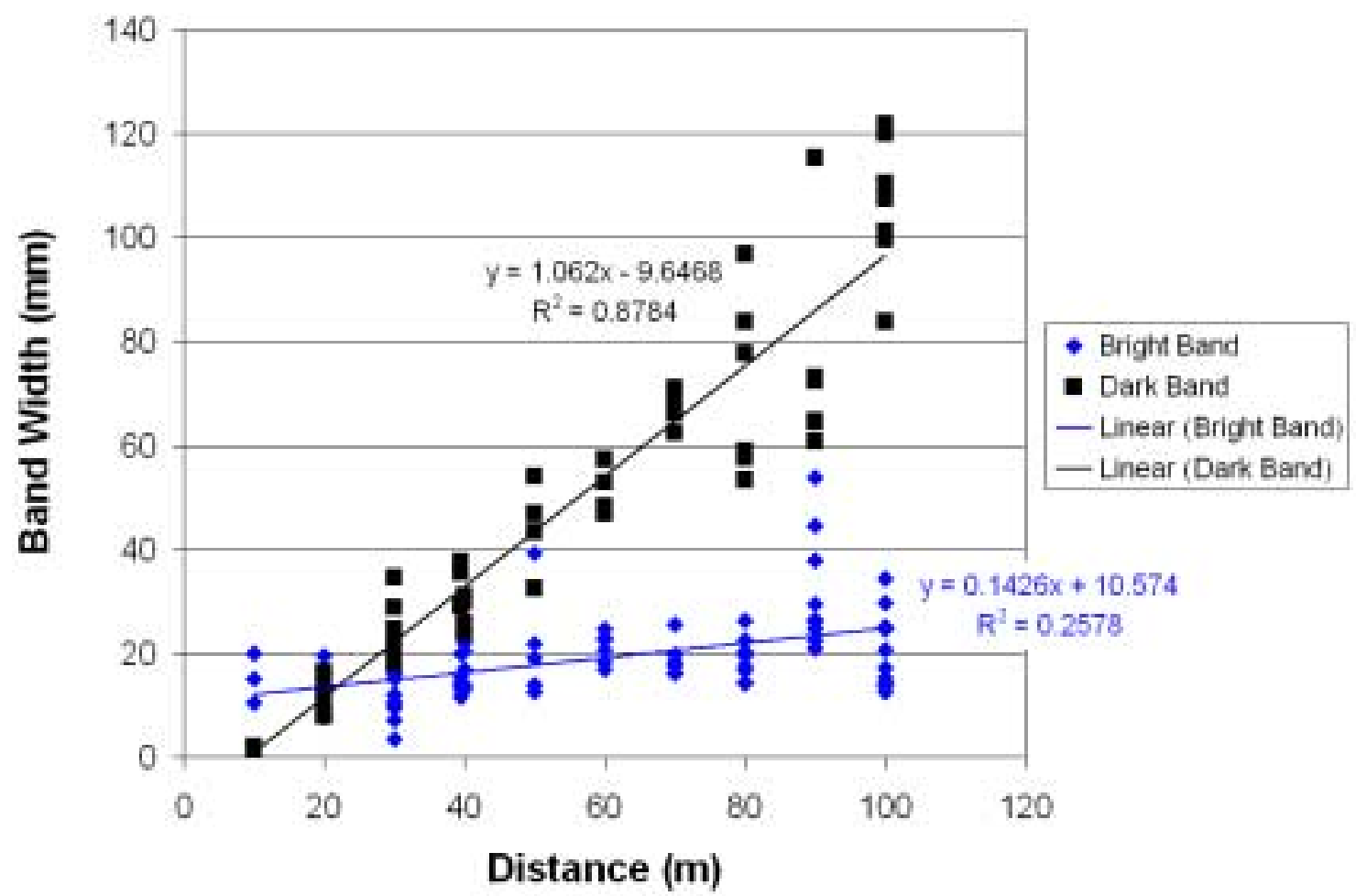

Figure 3.24. Combined Bright and Dark Band Widths vs. Distance.

Judging by the coefficients of determination for the two fits, the bright band shows a poorer fit than the dark band.

\subsection{Correlation}

The data obtained directly by the instrument are in the form of azimuth angle $(\theta)$, elevation angle $(\varphi)$, distance $(R)$. However, the only measured value is $R$ with the azimuth and elevation angles being obtained from line encoders. Therefore, when polar coordinates are converted to Cartesian 
coordinates by the instrument, nominal values (encoder values) of the azimuth and elevation angles are used. Thus, it is not possible to meaningfully evaluate cross-correlations [corr $(R, \theta)$, $\operatorname{corr}(R, \varphi)]$ and only the spatial correlation, corr $(R, R)$, can be investigated (Section 3.5.2).

\section{$\underline{3.5 .1 \quad T e m p o r a l ~ A u t o c o r r e l a t i o n ~-~ D a t a ~ S e t ~} 8$}

Assuming the 100 pseudo-replicated $^{\S}$ points taken at one location on the target represent a norm for replication through time for a measurement of the same (geographic) location under fixed operating conditions, it is possible to assess the (temporal) autocorrelation of the instrument by lagging each location's block of 100 points with respect to itself and computing the resulting autocorrelation function (ACF).

$$
r_{k}=\frac{\sum_{t=1}^{N-k}\left(x_{t}-\bar{x}\right)\left(x_{t+k}-\bar{x}\right)}{\sum_{t=1}^{N}\left(x_{t}-\bar{x}\right)^{2}}
$$

Doing this repeatedly across multiple blocks yields plots of the form in Fig. 3.26. In Fig. 3.26, in each frame, the X-axis represents LAG between the two versions of the same time sequence (LAG units coincide with the basic time sampling units) and the Y-axis represents the computed autocorrelation at the lag, i.e., $r_{k}$. The two sets of horizontal lines in each plot represents estimated $95 \%(2 \sigma)$ and $99 \%(3 \sigma)$ confidence bands for the autocorrelations. Plot plots outside the ranges of the indicated bounds would represent statistically significant nonzero autocorrelation(s) at $5 \%$ and $1 \%$ significance, respectively.

\footnotetext{
$\S$ True replication would involve zeroing out, re-setting, and re-measuring after a substantial time interval.
} 


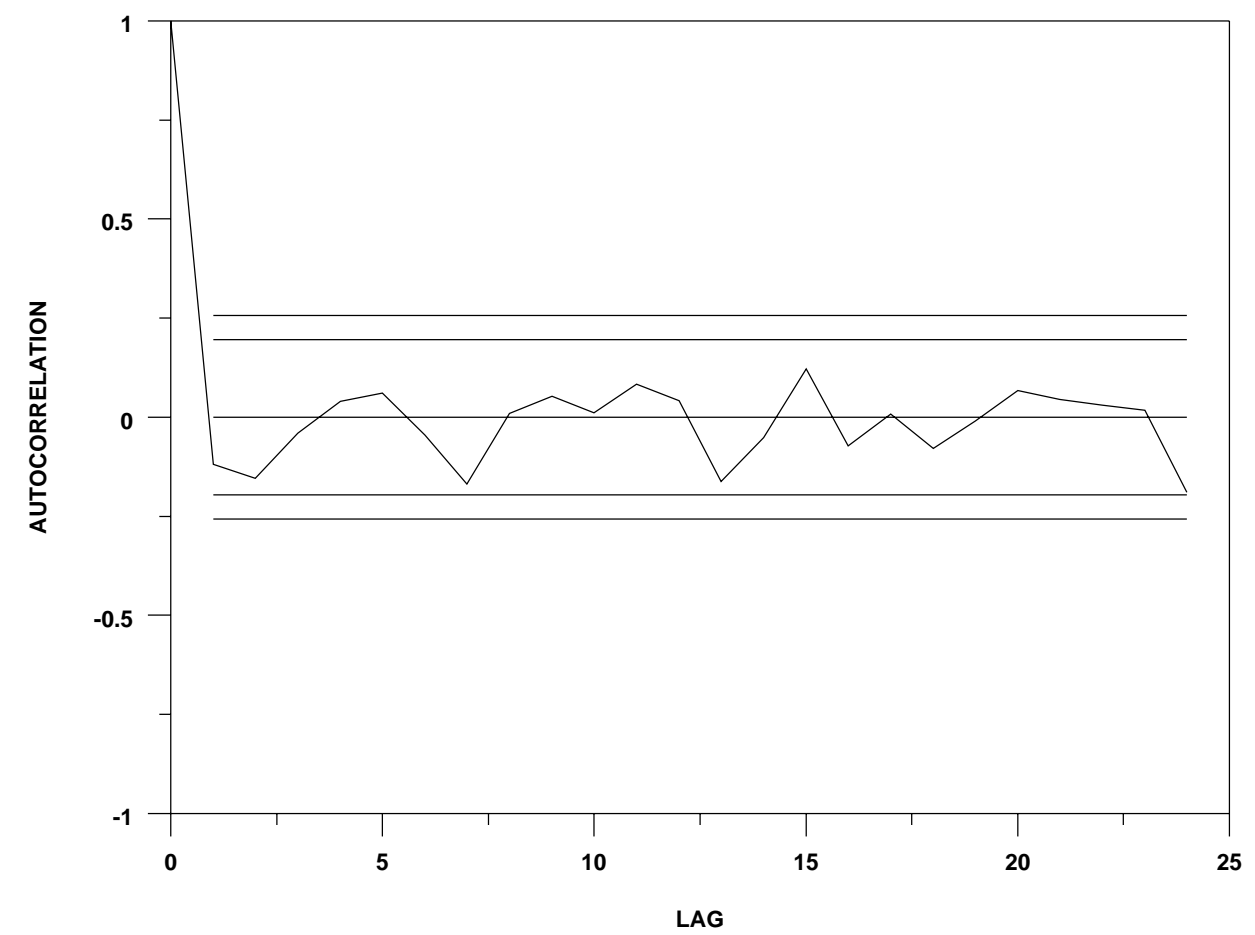

Figure 3.26. Autocorrelation Plots.

The plots in Fig. 3.26 display no significant autocorrelation at nontrivial $(\neq 1)$ lags. Significant low-order autocorrelation (lags 2,3,4) would be indicative of a stickiness in the replicated points: a tendency for their deviations about their mean to repeat/duplicate themselves at points close in time. Significant high-order autocorrelative structure would be indicative of a quasiperiodicity in the time sequence: a tendency for the sequence to duplicate, or to almost duplicate, itself at some fixed lag.

Autocorrelation causes a bias in the low, or optimistic, direction in variance estimates based on the replicated data. In fact, it is intuitive that the estimates will be biased low because of the tendency of autocorrelated points to self-adhere. Put in simplest terms, autocorrelation induces error in variance (and associated standard deviation) estimates. While the problem is correctable if suspected and detected ${ }^{* *}$, it is obviously preferable that the problem not occur at all. That this is the case here is reassuring.

\footnotetext{
${ }^{* *}$ An unbiased estimate of the variance can be obtained as the ordinate at zero frequency of the power spectrum
} 


\subsubsection{Spatial Autocorrelation - Data Set 7}

Data Set 7 is organized as 10 replicates per location at 25 vertically arranged locations along a vertical strip for 3 strips. The presence of the sequence of 10 repetitions at multiple contiguous/adjacent locations enables us to estimate spatial autocorrelation between adjacent, or closely spaced locations along the strip. The key variable in this experiment is the vertical distance between the points. The specified incremental angles between points are $0.045^{\circ}, 0.090^{\circ}$, and $0.180^{\circ}$ which translates to distances of $7.9 \mathrm{~mm}, 15.7 \mathrm{~mm}$, and $31.4 \mathrm{~mm}$ for a target located $10 \mathrm{~m}$ from the scanner.

The correlations $(\operatorname{lag}=1)$ for strips 1,2 and 3 and vertical incremental angles of $0.045^{\circ}, 0.090^{\circ}$, and $0.180^{\circ}$ are given in Tables 3.8, 3.9, and 3.10, respectively.

Table 3.8. Data Set 7: Spatial Correlation For $0.045^{\circ}$ Incremental Angle.

\begin{tabular}{|c|c|c|c|c|c|}
\hline \multicolumn{2}{|c|}{ Strip 1} & \multicolumn{2}{|c|}{ Strip 2} & \multicolumn{2}{|c|}{ Strip 3} \\
\hline R-R & Std. Error ${ }^{\dagger \dagger}$ & R-R & Std. Error ${ }^{\dagger \dagger}$ & R-R & Std. Error ${ }^{\dagger \dagger}$ \\
\hline-0.55204 & 0.25297 & 0.74432 & 0.19857 & -0.04289 & 0.34049 \\
\hline 0.07578 & 0.38314 & -0.17656 & 0.36967 & -0.03537 & 0.42698 \\
\hline 0.05171 & 0.39157 & 0.13766 & 0.43325 & 0.19172 & 0.21671 \\
\hline 0.27004 & 0.40448 & -0.28101 & 0.42454 & -0.44123 & 0.26475 \\
\hline-0.58448 & 0.1824 & -0.57393 & 0.34741 & -0.4847 & 0.29094 \\
\hline-0.37383 & 0.23536 & 0.35231 & 0.20785 & -0.45583 & 0.23539 \\
\hline 0.62769 & 0.20726 & 0.52773 & 0.15336 & -0.19037 & 0.33801 \\
\hline 0.50778 & 0.29949 & -0.14254 & 0.34996 & -0.01197 & 0.30406 \\
\hline-0.26985 & 0.40095 & -0.46646 & 0.32139 & 0.01148 & 0.38466 \\
\hline-0.13945 & 0.2607 & -0.16489 & 0.35893 & -0.42333 & 0.31418 \\
\hline 0.87244 & 0.09083 & -0.77195 & 0.17778 & 0.43474 & 0.2817 \\
\hline 0.08739 & 0.32706 & 0.26443 & 0.35237 & 0.36859 & 0.26577 \\
\hline-0.57979 & 0.21134 & -0.14849 & 0.33198 & -0.2936 & 0.28408 \\
\hline 0.91375 & 0.02805 & 0.78714 & 0.20364 & -0.32593 & 0.27716 \\
\hline 0.09417 & 0.30343 & 1.00000 & 0.00006 & 0.53441 & 0.19458 \\
\hline 0.82015 & 0.13774 & 1.00000 & 0.00004 & 0.1823 & 0.46205 \\
\hline-0.19088 & 0.32665 & -0.31894 & 0.26856 & -0.45655 & 0.23226 \\
\hline-0.38197 & 0.20382 & 0.39947 & 0.21379 & -0.54255 & 0.25972 \\
\hline 0.11923 & 0.34496 & 0.24458 & 0.36047 & 0.39676 & 0.29478 \\
\hline-0.35167 & 0.27788 & -0.5899 & 0.30531 & -0.10517 & 0.26977 \\
\hline-0.43707 & 0.23681 & -0.43439 & 0.35767 & -0.06267 & 0.30774 \\
\hline 0.82979 & 0.11297 & 0.04884 & 0.28561 & -0.09866 & 0.32113 \\
\hline-0.26641 & 0.37783 & -0.12623 & 0.27751 & -0.28201 & 0.23208 \\
\hline-0.33663 & 0.32035 & 0.35376 & 0.27308 & -0.0389 & 0.4825 \\
\hline
\end{tabular}

\footnotetext{
${ }^{\dagger}$ Standard errors for the correlations were estimated by bootstrapping [Efron and Tibshirani, 1993] .
} 
Table 3.9. Data Set 7: Spatial Correlation for $0.090^{\circ}$ Incremental Angle.

\begin{tabular}{|c|c|c|c|c|c|}
\hline \multicolumn{2}{|c|}{ Strip 1} & \multicolumn{2}{|c|}{ Strip 2} & \multicolumn{2}{|c|}{ Strip 3} \\
\hline R-R & Std. Error & R-R & Std. Error & R-R & Std. Error \\
\hline 0.04023 & 0.31993 & -0.37415 & 0.20074 & -0.3325 & 0.33687 \\
\hline-0.05737 & 0.38259 & 0.04083 & 0.32854 & 0.27579 & 0.41989 \\
\hline-0.44075 & 0.28008 & -0.40951 & 0.31755 & 0.6197 & 0.18361 \\
\hline-0.00014 & 0.32705 & 0.06147 & 0.36001 & 0.15189 & 0.29353 \\
\hline 0.77054 & 0.10097 & 0.04636 & 0.44261 & 0.50289 & 0.31596 \\
\hline-0.22626 & 0.25344 & -0.37949 & 0.30592 & 0.66239 & 0.21289 \\
\hline-0.43177 & 0.27858 & -0.11252 & 0.33779 & -0.14162 & 0.29372 \\
\hline 0.32753 & 0.30081 & 0.10783 & 0.39081 & 0.60301 & 0.28728 \\
\hline 0.62817 & 0.15617 & 0.44215 & 0.17788 & 0.58331 & 0.31369 \\
\hline 0.45546 & 0.33414 & -0.14143 & 0.35644 & 0.38173 & 0.2345 \\
\hline 0.09507 & 0.36422 & -0.58178 & 0.19458 & 0.48458 & 0.39203 \\
\hline-0.01288 & 0.42003 & 0.1496 & 0.33689 & -0.15646 & 0.28488 \\
\hline-0.23579 & 0.3064 & -0.09338 & 0.37834 & -0.22841 & 0.26505 \\
\hline-0.57032 & 0.22791 & 0.01072 & 0.3388 & -0.73704 & 0.46548 \\
\hline-0.28361 & 0.37853 & -0.2714 & 0.31101 & -0.31044 & 0.26085 \\
\hline-0.07629 & 0.36469 & -0.07577 & 0.31151 & 0.35164 & 0.26769 \\
\hline-0.01351 & 0.23284 & 0.0863 & 0.3234 & 0.33585 & 0.27064 \\
\hline 0.2583 & 0.34492 & 0.62126 & 0.23531 & 0.59442 & 0.15488 \\
\hline-0.56313 & 0.25592 & -0.28707 & 0.40308 & -0.25577 & 0.27133 \\
\hline 0.42167 & 0.35682 & -0.37826 & 0.20698 & -0.06747 & 0.29893 \\
\hline-0.75035 & 0.14136 & -0.75785 & 0.1334 & 0.21958 & 0.32173 \\
\hline-0.16274 & 0.23618 & -0.34097 & 0.34117 & -0.36802 & 0.33458 \\
\hline 0.06879 & 0.24266 & -0.09119 & 0.34036 & 0.13394 & 0.38527 \\
\hline 0.04338 & 0.29401 & 0.62354 & 0.29509 & -0.00936 & 0.44477 \\
\hline
\end{tabular}

Table 3.10. Data Set 7: Spatial Correlation for $0.180^{\circ}$ Incremental Angle.

\begin{tabular}{|c|c|c|c|c|c|}
\hline \multicolumn{2}{|c|}{ Strip 1 } & \multicolumn{2}{c|}{ Strip 2 } & \multicolumn{2}{c|}{ Strip 3 } \\
\hline R-R & Std. Error & R-R & Std. Error & R-R & Std. Error \\
\hline-0.02523 & 0.39223 & 0.3342 & 0.34421 & 0.44386 & 0.33459 \\
\hline-0.23081 & 0.37593 & -0.45636 & 0.35246 & 0.02245 & 0.40943 \\
\hline 0.03178 & 0.36891 & 0.45053 & 0.2358 & 0.00256 & 0.3951 \\
\hline 0.19884 & 0.23042 & 0.26613 & 0.42503 & -0.30439 & 0.36607 \\
\hline 0.04748 & 0.36262 & 0.42192 & 0.26559 & -0.18638 & 0.34745 \\
\hline-0.29251 & 0.261 & 0.33306 & 0.27872 & -0.1293 & 0.32023 \\
\hline-0.74941 & 0.27052 & -0.36537 & 0.2864 & -0.11169 & 0.35782 \\
\hline 0.18529 & 0.40528 & -0.33562 & 0.29819 & 0.67417 & 0.14484 \\
\hline
\end{tabular}




\begin{tabular}{|c|c|c|c|c|c|}
\hline \multicolumn{2}{|c|}{ Strip 1 } & \multicolumn{2}{c|}{ Strip 2 } & \multicolumn{2}{c|}{ Strip 3 } \\
\hline R-R & Std. Error & R-R & Std. Error & R-R & Std. Error \\
\hline 0.42923 & 0.4175 & 0.14002 & 0.42949 & -0.34621 & 0.30111 \\
\hline-0.58264 & 0.14779 & 0.32185 & 0.40692 & -0.17787 & 0.36141 \\
\hline 0.21543 & 0.33636 & 0.16281 & 0.35796 & 0.47939 & 0.24581 \\
\hline-0.55006 & 0.35867 & -0.19229 & 0.38524 & 0.47337 & 0.2233 \\
\hline-0.10123 & 0.31639 & -0.5181 & 0.23856 & 0.44582 & 0.36826 \\
\hline-0.01678 & 0.33585 & 0.17932 & 0.35488 & -0.40009 & 0.35683 \\
\hline-0.60713 & 0.20003 & -0.0475 & 0.39304 & 0.54936 & 0.26534 \\
\hline 0.01777 & 0.38883 & 0.32296 & 0.31779 & 0.02965 & 0.32261 \\
\hline 0.24499 & 0.29048 & 0.03808 & 0.2709 & -0.31793 & 0.25856 \\
\hline-0.10405 & 0.2983 & -0.18809 & 0.26094 & 0.2797 & 0.31558 \\
\hline-0.1687 & 0.47751 & 0.37456 & 0.18533 & 0.02767 & 0.29249 \\
\hline-0.57767 & 0.31923 & 0.03594 & 0.31149 & -0.53119 & 0.2245 \\
\hline-0.2125 & 0.35412 & -0.02728 & 0.38385 & 0.50402 & 0.28571 \\
\hline-0.15705 & 0.30546 & -0.49235 & 0.25695 & -0.20329 & 0.3737 \\
\hline 0.39246 & 0.41459 & 0.8526 & 0.07813 & -0.08847 & 0.27742 \\
\hline-0.40737 & 0.46504 & 0.12131 & 0.41772 & 0.21695 & 0.23713 \\
\hline Notes: Shaded cells are instances of significant correlation. & & & \\
\hline
\end{tabular}

Examination of the correlation values and associated standard errors ${ }^{\sharp \$}$ shows that there is sporadic, seemingly, random statistically significant correlation between contiguous measurements in a vertical strip. The number of incidences of significant spatial correlation (shaded cells in Tables 3.8 to 3.10) increases as the incremental angle decreases, i.e., the vertical distance between points decreases. Since the findings in this experiment are inconclusive, the existence of spatial autocorrelation will require further investigation. If spatial autocorrelation is present, simple propagation of error methods, that is, propagation of error that does not take $2^{\text {nd }}$ order, cross-correlation terms into account, may not yield reliable results.

$\$$ Double the standard errors and check to see if the interval correlation $\pm(2 \sigma)$ crosses zero. If it does, the correlation is statistically indistinguishable from zero at $95 \%$ confidence. 


\subsection{CONCLUSIONS AND RESEARCH NEEDS}

\subsection{Summary and Conclusions}

The potential applications of LADARs are numerous and they cross over several sectors of industry - construction, large-scale manufacturing, remote sensing, national defense (military). As the use of LADARs increases, there is a growing need to characterize the LADARs and to develop statistically justifiable uncertainties for the LADAR data and their end products. There have been only a handful of such efforts in this area.

To evaluate surface generation algorithms objectively, the accuracy/precision characteristics of the sensor must first be determined. Therefore, in the first phase of a NIST project, calibration experiments, varying distance/size/color/reflectivity of targets were conducted. In the second phase, evaluations of surface generation algorithms with respect to meshing, registration, data editing (filtering, handling outliers, etc.) were performed and are reported in Witzgall and Cheok [2001] and in a second report that is in preparation. In the third phase, all such knowledge has to be integrated into a credible calculation of statistical uncertainty for reconstructed scenes and derived values such as volume.

As part of the first phase, a series of exploratory experiments to characterize a laser scanner was conducted and these efforts are chronicled in this report. These experiments served three purposes: 1) assessment of the accuracy and precision of the range measurements, 2) learning process for the use of and familiarization with a LADAR, and 3) to address specific needs as they arose. The experimental parameters examined included target color, texture, reflectivity, angle of incidence, and beam divergence. The effects of target reflectivity and distance on the intensity value were examined to assess the possibility of using intensity values to identify objects within a scene.

The initial findings can be summarized as follows:

1. Accuracy:

a. For the instrument calibrated, for the two extreme colors (black and white) and for angle of incidences equal to $0^{\circ}$, the measurement errors (Data Set 5) generally fell within the $\pm 5 \mathrm{~cm}$ bounds (manufacturer's upper bounds).

b. Reduction of the laser output power (filter on laser) significantly decreased accuracy of a less reflective target (black) at the upper range of the LADAR.

2. Color Effect (Reflectivity):

a. Comparisons of the global regression line statistics show no dramatic color effect.

b. Less reflective targets (black, dark gray) are less precise at longer distances than more reflective targets (white).

c. Biases are also observed for some of the colors: a pink target is biased low. Dark gray and black targets are biased high.

d. Reduction of the laser output power (filter on laser) significantly decreased precision at the upper range of the LADAR regardless of target color. 
3. Angle of incidence:

a. As expected, the angle of incidence affects the range accuracy. Highly reflective targets (silver, shiny) yield the largest measurement errors for this series of experiments and are the least precise - for angles of incidence of $50^{\circ}$ and greater.

b. The least reflective target (black) sustains the most misses (no returns) as the angle of incidence increases.

4. LDP material (Highly reflective):

a. Consistently high $(>200)$ intensities for the entire range of the scanner was observed which makes the LDP material a potential candidate for the fabrication of bar codes.

b. Very significant measurement errors are observed for this material for distances less than $40 \mathrm{~m}$ due to saturation of the detector.

5. Autocorrelation:

a. No evidence of significant temporal autocorrelation was observed.

b. Random significant spatial correlations between contiguous measurements were observed and require further investigation.

These findings are based on a very limited number of experiments and on one type of laser scanner. It should be kept in mind that these findings are likely instrument dependent. Further experiments are needed to verify fully and expand upon these findings and to refine the calibration procedure.

The initial efforts and findings presented in this report provide a better understanding of what is required to calibrate similar sensors in terms of significant parameters affecting range measurements, test procedures, and calibration facility requirements. Additional experiments with other types of laser scanners would be necessary to develop standard calibration protocols appropriate for all or most of the commercially available scanners.

\subsection{Research Needs}

There are currently no facilities or standard test protocols for the calibration or performance evaluation of LADARs [Marshall et al. 2001]. As the potential application of LADARs grows, there is a need to establish a facility to calibrate and evaluate these types of sensors. It is anticipated that such a calibration facility would consist of an indoor and an outdoor facility. The indoor facility would allow for calibrations to be conducted in a controlled environment (temperature, humidity, pressure) while an outdoor facility would permit the evaluation of the scanner performance in a more realistic environment.

Test protocols and calibrations for the indoor facility would fall under two general categories:

- hardware calibration

- distance accuracy and precision

- pointing accuracy and precision

- laser beam divergence 
- depth and horizontal resolution - what is the minimum distance between objects that is detectable by the sensor

- minimum object size that is detectable by the sensor

- stability/degradation of sensor over time

- performance evaluation using artifacts - i.e., given several standard artifacts, how accurately and precisely can the following characteristics be determined:

- dimensions

- position/location

- area/volume

- geometry/object identity

In the second category, performance evaluation involves the assessment of the combination of hardware and software to produce a desired end product. LADAR data could be used "as is" (point cloud), but the full potential of such data lies in the use of the data to create 3D models from which positions, dimensions, areas, volumes, etc. can be ascertained. This process involves software to register multiple scans and to mesh the point cloud, as well as methods to clean/filter and to subsample the data. In general, the calibrations obtained from the first category above could be used for propagating instrument error through to the end product and to determine how to improve the scanner, whereas the calibrations in the second category would be used to measure and explicitly note the performance of the scanner. Metrics to evaluate the scanner performance are harder to establish than the metrics to evaluate the hardware accuracy and precision.

For each of the subtopics listed for hardware calibrations and performance evaluation, many issues have to be resolved. These issues include determining the optical characteristics of the standard targets (color, reflectivity, texture, etc.), shape, size, material, and placement of the artifacts, and choice of software package. Some hardware specific issues include developing methods to ensure that the target is perpendicular to the laser and procedures for dealing with instruments that are not setup for single point acquisition.

The outdoor facility would consist of designated areas that are open, partially wooded, and wooded. Permanent benchmarks would be located throughout the designated areas for accurately locating the sensor. In addition to allowing for the evaluation of the scanner under more realistic conditions, the outdoor facility would also allow for the evaluation of the sensor's ability to map terrain in different seasons - full foliage (summer), partial foliage (spring and fall), and no foliage (winter), and in different environmental conditions - sunny, cloudy, dusty, hot, cold. Artifacts could also be used in the outdoor facility.

The successful establishment of a LADAR calibration and the development of test protocols would require the participation, cooperation, and acceptance by the LADAR manufacturers and end users. This collaborative effort should start at the initial planning phases and continue throughout the whole process. The standardization of the test protocols would also require the inclusion of standards writing organizations such as American National Standards Institute (ANSI) and the American Society of Non-Destructive Testing. 


\section{REFERENCES}

Collier, R. [1998], "Characterization of a Range Scanning System Utilizing a Point Laser Rangefinder," MS Thesis, University of Tennessee, Knoxville, August, 74 pp.

Efron, B. and Tibshirani, R. J. [1993], An Introduction to the Bootstrap, Chapman and Hall, New York.

El-Hakim, S. F., Beraldin, J. A., and Blais, F. [1995], "A Comparative Evaluation of the Performance of Passive and Active 3-D Vision Systems," Proc. St. Petersburg Conference on Digital Photogrammetry, St. Petersburg, Russia, June 25-30, 1995. NRC 39160.

Kweon, I. S., Hoffman, R. and Krotkov [1991], "Experimental Characterization of the Perceptron Laser Rangefinder," CMU-RI-TR-91-1, The Robotics Institute, Carnegie Mellon University, Pittsburgh, PA, January, 44 pp.

Marshall, S. J., and Gilby, J. H. [2001], "New Opportunities in Non-Contact 3D Measurement," Proc. National Measurement Conf., 2001, Harrogate, UK.

Marshall, S. J., Whiteford, D. N., and Rixon, R. C. [2001], "Assessing the Performance of 3D Whole Body Imaging Systems," Proc. 6th Numérisation 3D/Scanning 2001 Congress, Paris, France.

Sachs, L. (1982), Applied Statistics: A Handbook of Techniques, New York, Springer-Verlag, p. 442.

Taylor, J. R. [1997], An Introduction to Error Analysis: The Study of Uncertainties in Physical Measurements, $2^{\text {nd }}$ ed., University Science Books, Sausalito, CA.

Witzgall, C. and Cheok, G. S. [2001], "Registering 3D Point Clouds: An Experimental Evaluation," NISTIR 6743, National Institute of Standards and Technology, Gaithersburg, MD, May, $41 \mathrm{pp}$. 


\section{APPENDIX A - Relative Distance}

Table A.1 Data Set 1 - Relative Distance Calibration: $0 \mathrm{~m}$ to $60 \mathrm{~m}$

\begin{tabular}{|c|c|c|c|c|c|}
\hline $\begin{array}{l}\text { Location: Envil } \\
\text { No Filter } \\
\text { Test Dates: July }\end{array}$ & $\begin{array}{l}\text { mentally ce } \\
4-15,1999\end{array}$ & olled lab, Blc & 220 & & el_dist_anal.xls \\
\hline $\begin{array}{c}\text { Interfero. Dist. } \\
(\mathrm{mm}) \\
\text { tall post }\end{array}$ & $\begin{array}{l}\text { Wht., not } \\
\text { shiny } \\
(\mathrm{m})\end{array}$ & $\begin{array}{l}\text { Interfero. } \\
\text { Dist. (mm) } \\
\text { short post }\end{array}$ & $\begin{array}{l}\text { Wht., not shiny } \\
\text { (m) }\end{array}$ & $\begin{array}{c}\text { Interfero. Dist. } \\
\text { (mm) } \\
\text { tall post }\end{array}$ & $\begin{array}{l}\text { Wht., shiny } \\
\text { (m) }\end{array}$ \\
\hline 0.00 & 4.315 & 0.00 & 4.468 & 0.00 & 4.588 \\
\hline 0.00 & 4.335 & 0.00 & 4.508 & 0.00 & 4.608 \\
\hline 0.00 & 4.395 & 0.00 & 4.468 & 0.00 & 4.608 \\
\hline 0.00 & 4.315 & 0.00 & 4.488 & 0.00 & 4.548 \\
\hline 0.00 & 4.315 & 0.00 & 4.488 & 0.00 & 4.608 \\
\hline 0.00 & 4.235 & 0.00 & 4.448 & 0.00 & 4.628 \\
\hline 0.00 & 4.295 & 0.00 & 4.448 & 0.00 & 4.588 \\
\hline 0.00 & 4.375 & 0.00 & 4.408 & 0.00 & 4.608 \\
\hline 0.00 & 4.415 & 0.00 & 4.408 & 0.00 & 4.608 \\
\hline 0.00 & 4.375 & 0.00 & 4.488 & 0.00 & 4.608 \\
\hline 0.00 & 4.370 & 0.00 & 4.488 & 0.00 & 4.568 \\
\hline 0.00 & 4.430 & 0.00 & 4.448 & 0.00 & 4.608 \\
\hline 0.00 & 4.410 & 0.00 & 4.488 & 0.00 & 4.608 \\
\hline 0.00 & 4.390 & 0.00 & 4.428 & 0.00 & 4.628 \\
\hline 0.00 & 4.450 & 0.00 & 4.408 & 0.00 & 4.588 \\
\hline 0.00 & 4.310 & 0.00 & 4.488 & 0.00 & 4.588 \\
\hline 0.00 & 4.370 & 0.00 & 4.548 & 0.00 & 4.628 \\
\hline 0.00 & 4.410 & 0.00 & 4.548 & 0.00 & 4.588 \\
\hline 0.00 & 4.410 & 0.00 & 4.408 & 0.00 & 4.548 \\
\hline 0.00 & 4.390 & 0.00 & 4.468 & 0.00 & 4.628 \\
\hline 0.00 & 4.370 & 0.00 & 4.508 & 0.00 & 4.568 \\
\hline 0.00 & 4.330 & 0.00 & 4.468 & 0.00 & 4.608 \\
\hline 0.00 & 4.370 & 0.00 & 4.488 & 0.00 & 4.608 \\
\hline 0.00 & 4.390 & 0.00 & 4.428 & 0.00 & 4.588 \\
\hline 0.00 & 4.430 & 0.00 & 4.428 & 0.00 & 4.608 \\
\hline 2047.17 & 6.312 & 2001.82 & 6.510 & 2000.02 & 6.630 \\
\hline 2047.17 & 6.427 & 2001.82 & 6.510 & 2000.02 & 6.59 \\
\hline 2047.17 & 6.347 & 2001.82 & 6.330 & 2000.02 & 6.63 \\
\hline 2047.17 & 6.407 & 2001.82 & 6.490 & 2000.02 & 6.59 \\
\hline 2047.17 & 6.407 & 2001.82 & 6.450 & 2000.02 & 6.57 \\
\hline 2047.17 & 6.367 & 2001.82 & 6.450 & 2000.02 & 6.61 \\
\hline 2047.17 & 6.367 & 2001.82 & 6.390 & 2000.02 & 6.63 \\
\hline 2047.17 & 6.387 & 2001.82 & 6.410 & 2000.02 & 6.61 \\
\hline 2047.17 & 6.507 & 2001.82 & 6.550 & 2000.02 & 6.61 \\
\hline 2047.17 & 6.387 & 2001.82 & 6.430 & 2000.02 & 6.55 \\
\hline 2047.17 & 6.387 & 2001.82 & 6.450 & 2000.02 & 6.57 \\
\hline 2047.17 & 6.407 & 2001.82 & 6.450 & 2000.02 & 6.61 \\
\hline 2047.17 & 6.503 & 2001.82 & 6.410 & 2000.02 & 6.59 \\
\hline 2047.17 & 6.307 & 2001.82 & 6.570 & 2000.02 & 6.63 \\
\hline 2047.17 & 6.447 & 2001.82 & 6.530 & 2000.02 & 6.59 \\
\hline 2047.17 & 6.427 & 2001.82 & 6.470 & 2000.02 & 6.63 \\
\hline 2047.17 & 6.263 & 2001.82 & 6.490 & 2000.02 & 6.61 \\
\hline 2047.17 & 6.463 & 2001.82 & 6.410 & 2000.02 & 6.59 \\
\hline 2047.17 & 6.423 & 2001.82 & 6.550 & 2000.02 & 6.65 \\
\hline 2047.17 & 6.403 & 2001.82 & 6.490 & 2000.02 & 6.63 \\
\hline 2047.17 & 6.363 & 2001.82 & 6.570 & 2000.02 & 6.63 \\
\hline 2047.17 & 6.363 & 2001.82 & 6.470 & 2000.02 & 6.63 \\
\hline 2047.17 & 6.483 & 2001.82 & 6.510 & 2000.02 & 6.63 \\
\hline 2047.17 & 6.383 & 2001.82 & 6.410 & 2000.02 & 6.63 \\
\hline 2047.17 & 6.423 & 2001.82 & 6.550 & 2000.02 & 6.63 \\
\hline 2047.17 & 6.423 & 4000.22 & 8.573 & 4000.21 & 8.593 \\
\hline 4005.47 & 8.266 & 4000.22 & 8.453 & 4000.21 & 8.573 \\
\hline 4005.47 & 8.226 & 4000.22 & 8.453 & 4000.21 & 8.593 \\
\hline 4005.47 & 8.266 & 4000.22 & 8.453 & 4000.21 & 8.573 \\
\hline 4005.47 & 8.246 & 4000.22 & 8.373 & 4000.21 & 8.593 \\
\hline
\end{tabular}


Table A.1 Data Set 1 - Relative Distance Calibration: $0 \mathrm{~m}$ to $60 \mathrm{~m}$

\begin{tabular}{|c|c|c|c|c|c|}
\hline \multicolumn{6}{|c|}{$\begin{array}{l}\text { Location: Environmentally controlled lab, Bldg } \\
\text { No Filter } \\
\text { Test Dates: July 14-15, } 1999\end{array}$} \\
\hline $\begin{array}{l}\text { Interfero. Dist. } \\
(\mathrm{mm}) \\
\text { tall post }\end{array}$ & $\begin{array}{l}\text { Wht., not } \\
\text { shiny } \\
\text { (m) }\end{array}$ & $\begin{array}{l}\text { Interfero. } \\
\text { Dist. (mm) } \\
\text { short post }\end{array}$ & $\begin{array}{l}\text { Wht., not shiny } \\
\text { (m) }\end{array}$ & $\begin{array}{l}\text { Interfero. Dist. } \\
\text { (mm) } \\
\text { tall post }\end{array}$ & $\begin{array}{l}\text { Wht., shiny } \\
\text { (m) }\end{array}$ \\
\hline \begin{tabular}{|l|}
4005.47 \\
\end{tabular} & 8.346 & 4000.22 & 8.493 & 4000.21 & 8.593 \\
\hline 4005.47 & 8.326 & 4000.22 & 8.453 & 4000.21 & 8.633 \\
\hline 4005.47 & 8.281 & 4000.22 & 8.493 & 4000.21 & 8.613 \\
\hline 4005.47 & 8.301 & 4000.22 & 8.633 & 4000.21 & 8.593 \\
\hline 4005.47 & 8.341 & 4000.22 & 8.593 & 4000.21 & 8.653 \\
\hline 4005.47 & 8.401 & 4000.22 & 8.493 & 4000.21 & 8.593 \\
\hline 4005.47 & 8.341 & 4000.22 & 8.433 & 4000.21 & 8.613 \\
\hline 4005.47 & 8.301 & 4000.22 & 8.493 & 4000.21 & 8.613 \\
\hline 4005.47 & 8.321 & 4000.22 & 8.433 & 4000.21 & 8.573 \\
\hline 4005.47 & 8.381 & 4000.22 & 8.493 & 4000.21 & 8.553 \\
\hline 4005.47 & 8.261 & 4000.22 & 8.453 & 4000.21 & 8.593 \\
\hline 4005.47 & 8.341 & 4000.22 & 8.453 & 4000.21 & 8.593 \\
\hline 4005.47 & 8.381 & 4000.22 & 8.473 & 4000.21 & 8.593 \\
\hline 4005.47 & 8.341 & 4000.22 & 8.493 & 4000.21 & 8.593 \\
\hline 4005.47 & 8.341 & 4000.22 & 8.353 & 4000.21 & 8.613 \\
\hline 4005.47 & 8.341 & 4000.22 & 8.373 & 4000.21 & 8.613 \\
\hline 4005.47 & 8.221 & 4000.22 & 8.573 & 4000.21 & 8.533 \\
\hline 4005.47 & 8.361 & 4000.22 & 8.533 & 4000.21 & 8.553 \\
\hline 4005.47 & 8.321 & 4000.22 & 8.613 & 4000.21 & 8.613 \\
\hline 4005.47 & 8.321 & 4000.22 & 8.493 & 4000.21 & 8.653 \\
\hline 4005.47 & 8.301 & 6002.26 & 10.496 & 6000.07 & 10.616 \\
\hline 4005.47 & 8.421 & 6002.26 & 10.536 & 6000.07 & 10.576 \\
\hline 6005.04 & 10.304 & 6002.26 & 10.636 & 6000.07 & 10.616 \\
\hline 6005.04 & 10.284 & 6002.26 & 10.636 & 6000.07 & 10.596 \\
\hline 6005.04 & 10.424 & 6002.26 & 10.536 & 6000.07 & 10.616 \\
\hline 6005.04 & 10.224 & 6002.26 & 10.676 & 6000.07 & 10.616 \\
\hline 6005.04 & 10.264 & 6002.26 & 10.596 & 6000.07 & 10.576 \\
\hline 6005.04 & 10.324 & 6002.26 & 10.556 & 6000.07 & 10.576 \\
\hline 6005.04 & 10.304 & 6002.26 & 10.616 & 6000.07 & 10.616 \\
\hline 6005.04 & 10.324 & 6002.26 & 10.436 & 6000.07 & 10.636 \\
\hline 6005.04 & 10.364 & 6002.26 & 10.536 & 6000.07 & 10.596 \\
\hline 6005.04 & 10.304 & 6002.26 & 10.476 & 6000.07 & 10.576 \\
\hline 6005.04 & 10.384 & 6002.26 & 10.456 & 6000.07 & 10.596 \\
\hline 6005.04 & 10.284 & 6002.26 & 10.456 & 6000.07 & 10.576 \\
\hline 6005.04 & 10.279 & 6002.26 & 10.476 & 6000.07 & 10.616 \\
\hline 6005.04 & 10.324 & 6002.26 & 10.476 & 6000.07 & 10.636 \\
\hline 6005.04 & 10.359 & 6002.26 & 10.556 & 6000.07 & 10.576 \\
\hline 6005.04 & 10.379 & 6002.26 & 10.476 & 6000.07 & 10.616 \\
\hline 6005.04 & 10.299 & 6002.26 & 10.436 & 6000.07 & 10.616 \\
\hline 6005.04 & 10.359 & 6002.26 & 10.596 & 6000.07 & 10.536 \\
\hline 6005.04 & 10.359 & 6002.26 & 10.536 & 6000.07 & 10.616 \\
\hline 6005.04 & 10.404 & 6002.26 & 10.516 & 6000.07 & 10.636 \\
\hline 6005.04 & 10.359 & 6002.26 & 10.496 & 6000.07 & 10.616 \\
\hline 6005.04 & 10.399 & 6002.26 & 10.576 & 6000.07 & 10.616 \\
\hline 6005.04 & 10.319 & 6002.26 & 10.456 & 6000.07 & 10.596 \\
\hline 6005.04 & 10.239 & 8001.15 & 12.580 & 8001.04 & 12.620 \\
\hline 6005.04 & 10.319 & 8001.15 & 12.459 & 8001.04 & 12.6 \\
\hline 8009.23 & 12.402 & 8001.15 & 12.479 & 8001.04 & 12.56 \\
\hline 8009.23 & 12.402 & 8001.15 & 12.439 & 8001.04 & 12.58 \\
\hline 8009.23 & 12.382 & 8001.15 & 12.500 & 8001.04 & 12.58 \\
\hline 8009.23 & 12.282 & 8001.15 & 12.439 & 8001.04 & 12.58 \\
\hline 8009.23 & 12.322 & 8001.15 & 12.580 & 8001.04 & 12.58 \\
\hline 8009.23 & 12.362 & 8001.15 & 12.620 & 8001.04 & 12.58 \\
\hline 8009.23 & 12.402 & 8001.15 & 12.540 & 8001.04 & 12.58 \\
\hline 8009.23 & 12.262 & 8001.15 & 12.479 & 8001.04 & 12.58 \\
\hline 8009.23 & 12.302 & 8001.15 & 12.559 & 8001.04 & 12.6 \\
\hline 8009.23 & 12.302 & 8001.15 & 12.500 & 8001.04 & 12.54 \\
\hline 8009.23 & 12.422 & 8001.15 & 12.479 & 8001.04 & 12.58 \\
\hline 8009.23 & 12.282 & 8001.15 & 12.540 & 8001.04 & 12.6 \\
\hline 8009.23 & 12.282 & 8001.15 & 12.520 & 8001.04 & 12.6 \\
\hline
\end{tabular}


Table A.1 Data Set 1 - Relative Distance Calibration: $0 \mathrm{~m}$ to $60 \mathrm{~m}$

\begin{tabular}{|c|c|c|c|c|c|}
\hline $\begin{array}{l}\text { Location: Envir } \\
\text { No Filter } \\
\text { Test Dates: July }\end{array}$ & $\begin{array}{l}\text { mentally cc } \\
-15,1999 \\
\end{array}$ & olled lab, Blc & 220 & & el_dist_anal.xls \\
\hline $\begin{array}{c}\text { Interfero. Dist. } \\
(\mathrm{mm}) \\
\text { tall post }\end{array}$ & $\begin{array}{l}\text { Wht., not } \\
\text { shiny } \\
\text { (m) }\end{array}$ & $\begin{array}{c}\text { Interfero. } \\
\text { Dist. (mm) } \\
\text { short post }\end{array}$ & $\begin{array}{l}\text { Wht., not shiny } \\
\text { (m) }\end{array}$ & $\begin{array}{l}\text { Interfero. Dist. } \\
(\mathrm{mm}) \\
\text { tall post }\end{array}$ & $\begin{array}{l}\text { Wht., shiny } \\
\text { (m) }\end{array}$ \\
\hline 8009.23 & 12.262 & 8001.15 & 12.540 & 8001.04 & 12.56 \\
\hline 8009.23 & 12.322 & 8001.15 & 12.500 & 8001.04 & 12.58 \\
\hline 8009.23 & 12.342 & 8001.15 & 12.540 & 8001.04 & 12.6 \\
\hline 8009.23 & 12.362 & 8001.15 & 12.479 & 8001.04 & 12.6 \\
\hline 8009.23 & 12.362 & 8001.15 & 12.540 & 8001.04 & 12.58 \\
\hline 8009.23 & 12.322 & 8001.15 & 12.540 & 8001.04 & 12.6 \\
\hline 8009.23 & 12.342 & 8001.15 & 12.439 & 8001.04 & 12.58 \\
\hline 8009.23 & 12.362 & 8001.15 & 12.620 & 8001.04 & 12.58 \\
\hline 8009.23 & 12.422 & 8001.15 & 12.560 & 8001.04 & 12.6 \\
\hline 8009.23 & 12.402 & 8001.15 & 12.379 & 8001.04 & 12.62 \\
\hline 8009.23 & 12.302 & 10000.28 & 14.584 & 10000.81 & 14.564 \\
\hline 8009.23 & 12.402 & 10000.28 & 14.524 & 10000.81 & 14.604 \\
\hline 10021.08 & 14.346 & 10000.28 & 14.483 & 10000.81 & 14.564 \\
\hline 10021.08 & 14.452 & 10000.28 & 14.584 & 10000.81 & 14.604 \\
\hline 10021.08 & 14.312 & 10000.28 & 14.584 & 10000.81 & 14.544 \\
\hline 10021.08 & 14.392 & 10000.28 & 14.564 & 10000.81 & 14.604 \\
\hline 10021.08 & 14.392 & 10000.28 & 14.504 & 10000.81 & 14.604 \\
\hline 10021.08 & 14.337 & 10000.28 & 14.564 & 10000.81 & 14.564 \\
\hline 10021.08 & 14.352 & 10000.28 & 14.504 & 10000.81 & 14.584 \\
\hline 10021.08 & 14.392 & 10000.28 & 14.483 & 10000.81 & 14.584 \\
\hline 10021.08 & 14.417 & 10000.28 & 14.524 & 10000.81 & 14.564 \\
\hline 10021.08 & 14.332 & 10000.28 & 14.584 & 10000.81 & 14.604 \\
\hline 10021.08 & 14.352 & 10000.28 & 14.544 & 10000.81 & 14.584 \\
\hline 10021.08 & 14.252 & 10000.28 & 14.483 & 10000.81 & 14.564 \\
\hline 10021.08 & 14.352 & 10000.28 & 14.564 & 10000.81 & 14.584 \\
\hline 10021.08 & 14.372 & 10000.28 & 14.604 & 10000.81 & 14.644 \\
\hline 10021.08 & 14.432 & 10000.28 & 14.624 & 10000.81 & 14.624 \\
\hline 10021.08 & 14.332 & 10000.28 & 14.524 & 10000.81 & 14.584 \\
\hline 10021.08 & 14.332 & 10000.28 & 14.504 & 10000.81 & 14.564 \\
\hline 10021.08 & 14.452 & 10000.28 & 14.524 & 10000.81 & 14.584 \\
\hline 10021.08 & 14.372 & 10000.28 & 14.564 & 10000.81 & 14.624 \\
\hline 10021.08 & 14.372 & 10000.28 & 14.544 & 10000.81 & 14.604 \\
\hline 10021.08 & 14.332 & 10000.28 & 14.504 & 10000.81 & 14.604 \\
\hline 10021.08 & 14.352 & 10000.28 & 14.604 & 10000.81 & 14.584 \\
\hline 10021.08 & 14.452 & 10000.28 & 14.584 & 10000.81 & 14.584 \\
\hline 10021.08 & 14.392 & 14997.95 & 19.425 & 15006.54 & 19.605 \\
\hline 10021.08 & 14.312 & 14997.95 & 19.445 & 15006.54 & 19.605 \\
\hline 15026.58 & 19.334 & 14997.95 & 19.485 & 15006.54 & 19.545 \\
\hline 15026.58 & 19.334 & 14997.95 & 19.445 & 15006.54 & 19.565 \\
\hline 15026.58 & 19.314 & 14997.95 & 19.425 & 15006.54 & 19.585 \\
\hline 15026.58 & 19.374 & 14997.95 & 19.525 & 15006.54 & 19.585 \\
\hline 15026.58 & 19.314 & 14997.95 & 19.405 & 15006.54 & 19.545 \\
\hline 15026.58 & 19.394 & 14997.95 & 19.425 & 15006.54 & 19.605 \\
\hline 15026.58 & 19.334 & 14997.95 & 19.454 & 15006.54 & 19.585 \\
\hline 15026.58 & 19.274 & 14997.95 & 19.425 & 15006.54 & 19.585 \\
\hline 15026.58 & 19.374 & 14997.95 & 19.425 & 15006.54 & 19.565 \\
\hline 15026.58 & 19.374 & 14997.95 & 19.445 & 15006.54 & 19.585 \\
\hline 15026.58 & 19.374 & 14997.95 & 19.485 & 15006.54 & 19.605 \\
\hline 15026.58 & 19.354 & 14997.95 & 19.445 & 15006.54 & 19.545 \\
\hline 15026.58 & 19.334 & 14997.95 & 19.485 & 15006.54 & 19.585 \\
\hline 15026.58 & 19.394 & 14997.95 & 19.485 & 15006.54 & 19.585 \\
\hline 15026.58 & 19.294 & 14997.95 & 19.425 & 15006.54 & 19.605 \\
\hline 15026.58 & 19.334 & 14997.95 & 19.485 & 15006.54 & 19.545 \\
\hline 15026.58 & 19.334 & 14997.95 & 19.425 & 15006.54 & 19.545 \\
\hline 15026.58 & 19.354 & 14997.95 & 19.465 & 15006.54 & 19.585 \\
\hline 15026.58 & 19.294 & 14997.95 & 19.405 & 15006.54 & 19.545 \\
\hline 15026.58 & 19.354 & 14997.95 & 19.485 & 15006.54 & 19.565 \\
\hline 15026.58 & 19.414 & 14997.95 & 19.425 & 15006.54 & 19.585 \\
\hline 15026.58 & 19.294 & 14997.95 & 19.405 & 15006.54 & 19.545 \\
\hline 15026.58 & 19.294 & 14997.95 & 19.445 & 15006.54 & 19.585 \\
\hline
\end{tabular}


Table A.1 Data Set 1 - Relative Distance Calibration: $0 \mathrm{~m}$ to $60 \mathrm{~m}$

\begin{tabular}{|c|c|c|c|c|c|}
\hline $\begin{array}{l}\text { Location: Envil } \\
\text { No Filter } \\
\text { Test Dates: July }\end{array}$ & $\begin{array}{r}\text { mentally cc } \\
-15,1999 \\
\end{array}$ & olled lab, Blc & 220 & & el_dist_anal.xls \\
\hline $\begin{array}{c}\text { Interfero. Dist. } \\
(\mathrm{mm}) \\
\text { tall post }\end{array}$ & $\begin{array}{l}\text { Wht., not } \\
\text { shiny } \\
\text { (m) }\end{array}$ & $\begin{array}{l}\text { Interfero. } \\
\text { Dist. (mm) } \\
\text { short post }\end{array}$ & $\begin{array}{l}\text { Wht.,not shiny } \\
\text { (m) }\end{array}$ & $\begin{array}{c}\text { Interfero. Dist. } \\
(\mathrm{mm}) \\
\text { tall post }\end{array}$ & $\begin{array}{l}\text { Wht., shiny } \\
\text { (m) }\end{array}$ \\
\hline 15026.58 & 19.394 & 20010.64 & 24.425 & 20136.28 & 24.725 \\
\hline 15026.58 & 19.274 & 20010.64 & 24.505 & 20136.28 & 24.745 \\
\hline 20015.67 & 24.274 & 20010.64 & 24.505 & 20136.28 & 24.685 \\
\hline 20015.67 & 24.31 & 20010.64 & 24.565 & 20136.28 & 24.725 \\
\hline 20015.67 & 24.27 & 20010.64 & 24.505 & 20136.28 & 24.705 \\
\hline 20015.67 & 24.29 & 20010.64 & 24.485 & 20136.28 & 24.685 \\
\hline 20015.67 & 24.25 & 20010.64 & 24.555 & 20136.28 & 24.725 \\
\hline 20015.67 & 24.35 & 20010.64 & 24.485 & 20136.28 & 24.725 \\
\hline 20015.67 & 24.29 & 20010.64 & 24.445 & 20136.28 & 24.765 \\
\hline 20015.67 & 24.31 & 20010.64 & 24.445 & 20136.28 & 24.725 \\
\hline 20015.67 & 24.33 & 20010.64 & 24.405 & 20136.28 & 24.725 \\
\hline 20015.67 & 24.31 & 20010.64 & 24.445 & 20136.28 & 24.705 \\
\hline 20015.67 & 24.33 & 20010.64 & 24.525 & 20136.28 & 24.705 \\
\hline 20015.67 & 24.29 & 20010.64 & 24.445 & 20136.28 & 24.705 \\
\hline 20015.67 & 24.31 & 20010.64 & 24.425 & 20136.28 & 24.685 \\
\hline 20015.67 & 24.33 & 20010.64 & 24.465 & 20136.28 & 24.705 \\
\hline 20015.67 & 24.27 & 20010.64 & 24.505 & 20136.28 & 24.765 \\
\hline 20015.67 & 24.31 & 20010.64 & 24.525 & 20136.28 & 24.665 \\
\hline 20015.67 & 24.31 & 20010.64 & 24.485 & 20136.28 & 24.685 \\
\hline 20015.67 & 24.27 & 20010.64 & 24.545 & 20136.28 & 24.685 \\
\hline 20015.67 & 24.33 & 20010.64 & 24.505 & 20136.28 & 24.705 \\
\hline 20015.67 & 24.29 & 20010.64 & 24.485 & 20136.28 & 24.685 \\
\hline 20015.67 & 24.33 & 20010.64 & 24.485 & 20136.28 & 24.705 \\
\hline 20015.67 & 24.37 & 20010.64 & 24.565 & 20136.28 & 24.725 \\
\hline 20015.67 & 24.31 & 20010.64 & 24.545 & 20136.28 & 24.725 \\
\hline 20015.67 & 24.29 & 30007.36 & 34.445 & 30806.36 & 35.385 \\
\hline 20015.67 & 24.25 & 30007.36 & 34.505 & 30806.36 & 35.325 \\
\hline 29936.1 & 34.250 & 30007.36 & 34.465 & 30806.36 & 35.365 \\
\hline 29936.1 & 34.23 & 30007.36 & 34.545 & 30806.36 & 35.365 \\
\hline 29936.1 & 34.23 & 30007.36 & 34.465 & 30806.36 & 35.365 \\
\hline 29936.1 & 34.21 & 30007.36 & 34.545 & 30806.36 & 35.385 \\
\hline 29936.1 & 34.21 & 30007.36 & 34.465 & 30806.36 & 35.325 \\
\hline 29936.1 & 34.23 & 30007.36 & 34.465 & 30806.36 & 35.465 \\
\hline 29936.1 & 34.19 & 30007.36 & 34.485 & 30806.36 & 35.365 \\
\hline 29936.1 & 34.23 & 30007.36 & 34.505 & 30806.36 & 35.345 \\
\hline 29936.1 & 34.21 & 30007.36 & 34.465 & 30806.36 & 35.385 \\
\hline 29936.1 & 34.27 & 30007.36 & 34.505 & 30806.36 & 35.325 \\
\hline 29936.1 & 34.19 & 30007.36 & 34.485 & 30806.36 & 35.425 \\
\hline 29936.1 & 34.17 & 30007.36 & 34.485 & 30806.36 & 35.365 \\
\hline 29936.1 & 34.23 & 30007.36 & 34.525 & 30806.36 & 35.385 \\
\hline 29936.1 & 34.21 & 30007.36 & 34.505 & 30806.36 & 35.385 \\
\hline 29936.1 & 34.21 & 30007.36 & 34.485 & 30806.36 & 35.385 \\
\hline 29936.1 & 34.23 & 30007.36 & 34.465 & 30806.36 & 35.345 \\
\hline 29936.1 & 34.21 & 30007.36 & 34.485 & 30806.36 & 35.385 \\
\hline 29936.1 & 34.19 & 30007.36 & 34.465 & 30806.36 & 35.365 \\
\hline 29936.1 & 34.23 & 30007.36 & 34.505 & 30806.36 & 35.385 \\
\hline 29936.1 & 34.27 & 30007.36 & 34.385 & 30806.36 & 35.385 \\
\hline 29936.1 & 34.19 & 30007.36 & 34.325 & 30806.36 & 35.385 \\
\hline 29936.1 & 34.23 & 30007.36 & 34.505 & 30806.36 & 35.385 \\
\hline 29936.1 & 34.23 & 30007.36 & 34.485 & 30806.36 & 35.365 \\
\hline 29936.1 & 34.23 & 0.00 & 4.468 & 39913.22 & 44.425 \\
\hline 29936.1 & 34.25 & 0.00 & 4.508 & 39913.22 & 44.425 \\
\hline 39998.52 & 44.870 & 0.00 & 4.528 & 39913.22 & 44.445 \\
\hline 39998.52 & 44.89 & 0.00 & 4.508 & 39913.22 & 44.465 \\
\hline 39998.52 & 44.89 & 0.00 & 4.488 & 39913.22 & 44.445 \\
\hline 39998.52 & 44.83 & 0.00 & 4.548 & 39913.22 & 44.465 \\
\hline 39998.52 & 44.87 & 0.00 & 4.468 & 39913.22 & 44.425 \\
\hline 39998.52 & 44.805 & 0.00 & 4.488 & 39913.22 & 44.445 \\
\hline 39998.52 & 44.865 & 0.00 & 4.468 & 39913.22 & 44.465 \\
\hline 39998.52 & 44.85 & 0.00 & 4.388 & 39913.22 & 44.465 \\
\hline
\end{tabular}


Table A.1 Data Set 1 - Relative Distance Calibration: $0 \mathrm{~m}$ to $60 \mathrm{~m}$

\begin{tabular}{|c|c|c|c|c|c|}
\hline $\begin{array}{l}\text { Location: Envil } \\
\text { No Filter } \\
\text { Test Dates: July }\end{array}$ & $\begin{array}{l}\text { mentally cc } \\
-15,1999\end{array}$ & olled lab, Blc & 220 & & el_dist_anal.xls \\
\hline $\begin{array}{c}\text { Interfero. Dist. } \\
(\mathrm{mm}) \\
\text { tall post }\end{array}$ & $\begin{array}{l}\text { Wht., not } \\
\text { shiny } \\
\text { (m) }\end{array}$ & $\begin{array}{l}\text { Interfero. } \\
\text { Dist. (mm) } \\
\text { short post }\end{array}$ & $\begin{array}{l}\text { Wht., not shiny } \\
\text { (m) }\end{array}$ & $\begin{array}{c}\text { Interfero. Dist. } \\
(\mathrm{mm}) \\
\text { tall post }\end{array}$ & $\begin{array}{l}\text { Wht., shiny } \\
\text { (m) }\end{array}$ \\
\hline 39998.52 & 44.87 & 0.00 & 4.428 & 39913.22 & 44.445 \\
\hline 39998.52 & 44.87 & 0.00 & 4.528 & 39913.22 & 44.465 \\
\hline 39998.52 & 44.81 & 0.00 & 4.488 & 39913.22 & 44.425 \\
\hline 39998.52 & 44.83 & 0.00 & 4.488 & 39913.22 & 44.445 \\
\hline 39998.52 & 44.81 & 0.00 & 4.468 & 39913.22 & 44.445 \\
\hline 39998.52 & 44.89 & 0.00 & 4.448 & 39913.22 & 44.465 \\
\hline 39998.52 & 44.85 & 0.00 & 4.448 & 39913.22 & 44.465 \\
\hline 39998.52 & 44.85 & 0.00 & 4.488 & 39913.22 & 44.465 \\
\hline 39998.52 & 44.85 & 0.00 & 4.488 & 39913.22 & 44.465 \\
\hline 39998.52 & 44.91 & 0.00 & 4.488 & 39913.22 & 44.405 \\
\hline 39998.52 & 44.89 & 0.00 & 4.388 & 39913.22 & 44.465 \\
\hline 39998.52 & 44.83 & 0.00 & 4.548 & 39913.22 & 44.465 \\
\hline 39998.52 & 44.85 & 0.00 & 4.508 & 39913.22 & 44.425 \\
\hline 39998.52 & 44.83 & 0.00 & 4.568 & 39913.22 & 44.425 \\
\hline 39998.52 & 44.83 & 0.00 & 4.528 & 39913.22 & 44.445 \\
\hline 39998.52 & 44.87 & 39995.68 & 44.465 & 39913.22 & 44.445 \\
\hline 39998.52 & 44.85 & 39995.68 & 44.545 & 49895.7 & 54.465 \\
\hline 49954.58 & 54.505 & 39995.68 & 44.465 & 49895.7 & 54.485 \\
\hline 49954.58 & 54.525 & 39995.68 & 44.505 & 49895.7 & 54.485 \\
\hline 49954.58 & 54.565 & 39995.68 & 44.485 & 49895.7 & 54.445 \\
\hline 49954.58 & 54.605 & 39995.68 & 44.465 & 49895.7 & 54.465 \\
\hline 49954.58 & 54.485 & 39995.68 & 44.465 & 49895.7 & 54.445 \\
\hline 49954.58 & 54.605 & 39995.68 & 44.505 & 49895.7 & 54.465 \\
\hline 49954.58 & 54.565 & 39995.68 & 44.525 & 49895.7 & 54.485 \\
\hline 49954.58 & 54.525 & 39995.68 & 44.465 & 49895.7 & 54.465 \\
\hline 49954.58 & 54.465 & 39995.68 & 44.425 & 49895.7 & 54.445 \\
\hline 49954.58 & 54.505 & 39995.68 & 44.505 & 49895.7 & 54.445 \\
\hline 49954.58 & 54.565 & 39995.68 & 44.445 & 49895.7 & 54.445 \\
\hline 49954.58 & 54.585 & 39995.68 & 44.485 & 49895.7 & 54.465 \\
\hline 49954.58 & 54.525 & 39995.68 & 44.505 & 49895.7 & 54.465 \\
\hline 49954.58 & 54.545 & 39995.68 & 44.525 & 49895.7 & 54.445 \\
\hline 49954.58 & 54.545 & 39995.68 & 44.585 & 49895.7 & 54.445 \\
\hline 49954.58 & 54.545 & 39995.68 & 44.505 & 49895.7 & 54.465 \\
\hline 49954.58 & 54.505 & 39995.68 & 44.585 & 49895.7 & 54.465 \\
\hline 49954.58 & 54.565 & 39995.68 & 44.505 & 49895.7 & 54.445 \\
\hline 49954.58 & 54.525 & 39995.68 & 44.565 & 49895.7 & 54.445 \\
\hline 49954.58 & 54.465 & 39995.68 & 44.505 & 49895.7 & 54.445 \\
\hline 49954.58 & 54.565 & 39995.68 & 44.565 & 49895.7 & 54.445 \\
\hline 49954.58 & 54.545 & 39995.68 & 44.585 & 49895.7 & 54.465 \\
\hline 49954.58 & 54.625 & 39995.68 & 44.565 & 49895.7 & 54.465 \\
\hline 49954.58 & 54.545 & 49951.73 & 54.365 & 49895.7 & 54.485 \\
\hline 49954.58 & 54.505 & 49951.73 & 54.405 & 49895.7 & 54.465 \\
\hline & & 49951.73 & 54.465 & & \\
\hline & & 49951.73 & 54.445 & & \\
\hline & & 49951.73 & 54.425 & & \\
\hline & & 49951.73 & 54.465 & & \\
\hline & & 49951.73 & 54.425 & & \\
\hline & & 49951.73 & 54.445 & & \\
\hline & & 49951.73 & 54.445 & & \\
\hline & & 49951.73 & 54.385 & & \\
\hline & & 49951.73 & 54.365 & & \\
\hline & & 49951.73 & 54.445 & & \\
\hline & & 49951.73 & 54.405 & & \\
\hline & & 49951.73 & 54.405 & & \\
\hline & & 49951.73 & 54.425 & & \\
\hline & & 49951.73 & 54.465 & & \\
\hline & & 49951.73 & 54.405 & & \\
\hline & & 49951.73 & 54.425 & & \\
\hline & & 49951.73 & 54.425 & & \\
\hline & & 49951.73 & 54.445 & & \\
\hline
\end{tabular}


Table A.1 Data Set 1 - Relative Distance Calibration: $0 \mathrm{~m}$ to $60 \mathrm{~m}$ Location: Environmentally controlled lab, Bldg. 220

File: rel_dist_anal.xls No Filter

Test Dates: July 14-15, 1999

\begin{tabular}{|c|c|c|c|c|c|}
$\begin{array}{c}\text { Interfero. Dist. } \\
(\mathrm{mm})\end{array}$ & $\begin{array}{c}\text { Wht., not } \\
\text { shiny } \\
\text { tall post }\end{array}$ & $\begin{array}{c}\text { Interfero. } \\
\text { Dist. (m) } \\
\text { short post }\end{array}$ & $\begin{array}{c}\text { Wht.,not shiny } \\
(\mathrm{m})\end{array}$ & $\begin{array}{c}\text { Interfero. Dist. } \\
(\mathrm{mm}) \\
\text { tall post }\end{array}$ & $\begin{array}{c}\text { Wht., shiny } \\
(\mathrm{m})\end{array}$ \\
& & 49951.73 & 54.425 & & \\
& & 49951.73 & 54.425 & & \\
& & 49951.73 & 54.465 & & \\
& & 49951.73 & 54.565 & & \\
& & 49951.73 & 54.505 & & \\
& & &
\end{tabular}


Table A.1 Data Set1 - Relative Distance Calibration: $0 \mathrm{~m}$ to $60 \mathrm{~m}$

\begin{tabular}{|c|c|c|c|c|c|c|c|}
\hline \multicolumn{8}{|c|}{$\begin{array}{l}\text { Location: Environmentally controlled } \\
\text { No Filter } \\
\text { Test dates: July } 14-15,1999\end{array}$} \\
\hline $\begin{array}{l}\text { Interfero. } \\
\text { Dist. } \\
(\mathrm{mm})\end{array}$ & $\begin{array}{l}\text { Blck, not } \\
\text { shiny } \\
\text { (m) }\end{array}$ & $\begin{array}{l}\text { Interfero. } \\
\text { Dist. } \\
\text { (mm) }\end{array}$ & $\begin{array}{c}\text { Blck, shiny } \\
\text { (m) }\end{array}$ & $\begin{array}{l}\text { Interfero. } \\
\text { Dist. } \\
\text { (mm) }\end{array}$ & $\begin{array}{l}\text { Dk. Grey, } \\
\text { rough } \\
\text { (m) }\end{array}$ & $\begin{array}{l}\text { Interfero. } \\
\text { Dist. } \\
(\mathrm{mm})\end{array}$ & $\begin{array}{l}\text { Pink, not } \\
\text { shiny } \\
\text { (m) }\end{array}$ \\
\hline 0 & 4.428 & 0 & 4.428 & 0 & 4.468 & 0 & 4.548 \\
\hline 0 & 4.428 & 0 & 4.388 & 0 & 4.408 & 0 & 4.528 \\
\hline 0 & 4.408 & 0 & 4.408 & 0 & 4.408 & 0 & 4.528 \\
\hline 0 & 4.408 & 0 & 4.408 & 0 & 4.428 & 0 & 4.488 \\
\hline 0 & 4.488 & 0 & 4.408 & 0 & 4.408 & 0 & 4.488 \\
\hline 0 & 4.368 & 0 & 4.408 & 0 & 4.388 & 0 & 4.548 \\
\hline 0 & 4.388 & 0 & 4.408 & 0 & 4.428 & 0 & 4.508 \\
\hline 0 & 4.408 & 0 & 4.368 & 0 & 4.388 & 0 & 4.528 \\
\hline 0 & 4.388 & 0 & 4.428 & 0 & 4.448 & 0 & 4.528 \\
\hline 0 & 4.388 & 0 & 4.428 & 0 & 4.428 & 0 & 4.548 \\
\hline 0 & 4.408 & 0 & 4.428 & 0 & 4.428 & 0 & 4.508 \\
\hline 0 & 4.408 & 0 & 4.428 & 0 & 4.408 & 0 & 4.568 \\
\hline 0 & 4.408 & 0 & 4.408 & 0 & 4.408 & 0 & 4.468 \\
\hline 0 & 4.408 & 0 & 4.388 & 0 & 4.428 & 0 & 4.528 \\
\hline 0 & 4.388 & 0 & 4.428 & 0 & 4.368 & 0 & 4.508 \\
\hline 0 & 4.408 & 0 & 4.408 & 0 & 4.408 & 0 & 4.548 \\
\hline 0 & 4.408 & 0 & 4.348 & 0 & 4.408 & 0 & 4.528 \\
\hline 0 & 4.388 & 0 & 4.428 & 0 & 4.388 & 0 & 4.528 \\
\hline 0 & 4.388 & 0 & 4.428 & 0 & 4.408 & 0 & 4.508 \\
\hline 0 & 4.388 & 0 & 4.408 & 0 & 4.428 & 0 & 4.528 \\
\hline 0 & 4.388 & 0 & 4.428 & 0 & 4.428 & 0 & 4.528 \\
\hline 0 & 4.408 & 0 & 4.368 & 0 & 4.428 & 0 & 4.488 \\
\hline 0 & 4.388 & 0 & 4.428 & 0 & 4.408 & 0 & 4.468 \\
\hline 0 & 4.408 & 0 & 4.408 & 0 & 4.428 & 0 & 4.508 \\
\hline 0 & 4.408 & 0 & 4.408 & 0 & 4.428 & 0 & 4.528 \\
\hline 2000.24 & 6.430 & 2000.57 & 6.410 & 2001.41 & 6.410 & 2000.71 & 6.530 \\
\hline 2000.24 & 6.410 & 2000.57 & 6.470 & 2001.41 & 6.450 & 2000.71 & 6.470 \\
\hline 2000.24 & 6.410 & 2000.57 & 6.470 & 2001.41 & 6.450 & 2000.71 & 6.490 \\
\hline 2000.24 & 6.410 & 2000.57 & 6.470 & 2001.41 & 6.470 & 2000.71 & 6.490 \\
\hline 2000.24 & 6.370 & 2000.57 & 6.450 & 2001.41 & 6.430 & 2000.71 & 6.530 \\
\hline 2000.24 & 6.390 & 2000.57 & 6.430 & 2001.41 & 6.430 & 2000.71 & 6.530 \\
\hline 2000.24 & 6.390 & 2000.57 & 6.430 & 2001.41 & 6.470 & 2000.71 & 6.510 \\
\hline 2000.24 & 6.450 & 2000.57 & 6.410 & 2001.41 & 6.450 & 2000.71 & 6.470 \\
\hline 2000.24 & 6.430 & 2000.57 & 6.450 & 2001.41 & 6.490 & 2000.71 & 6.490 \\
\hline 2000.24 & 6.370 & 2000.57 & 6.410 & 2001.41 & 6.470 & 2000.71 & 6.510 \\
\hline 2000.24 & 6.390 & 2000.57 & 6.430 & 2001.41 & 6.410 & 2000.71 & 6.530 \\
\hline 2000.24 & 6.390 & 2000.57 & 6.470 & 2001.41 & 6.470 & 2000.71 & 6.510 \\
\hline 2000.24 & 6.410 & 2000.57 & 6.450 & 2001.41 & 6.430 & 2000.71 & 6.530 \\
\hline 2000.24 & 6.430 & 2000.57 & 6.470 & 2001.41 & 6.430 & 2000.71 & 6.510 \\
\hline 2000.24 & 6.410 & 2000.57 & 6.430 & 2001.41 & 6.490 & 2000.71 & 6.510 \\
\hline 2000.24 & 6.410 & 2000.57 & 6.430 & 2001.41 & 6.430 & 2000.71 & 6.490 \\
\hline 2000.24 & 6.410 & 2000.57 & 6.470 & 2001.41 & 6.470 & 2000.71 & 6.530 \\
\hline 2000.24 & 6.390 & 2000.57 & 6.450 & 2001.41 & 6.490 & 2000.71 & 6.490 \\
\hline 2000.24 & 6.410 & 2000.57 & 6.450 & 2001.41 & 6.430 & 2000.71 & 6.510 \\
\hline 2000.24 & 6.430 & 2000.57 & 6.450 & 2001.41 & 6.430 & 2000.71 & 6.510 \\
\hline 2000.24 & 6.430 & 2000.57 & 6.450 & 2001.41 & 6.450 & 2000.71 & 6.490 \\
\hline 2000.24 & 6.390 & 2000.57 & 6.410 & 2001.41 & 6.390 & 2000.71 & 6.550 \\
\hline 2000.24 & 6.430 & 2000.57 & 6.450 & 2001.41 & 6.430 & 2000.71 & 6.490 \\
\hline 2000.24 & 6.410 & 2000.57 & 6.430 & 2001.41 & 6.430 & 2000.71 & 6.550 \\
\hline 2000.24 & 6.430 & 2000.57 & 6.470 & 2001.41 & 6.470 & 2000.71 & 6.510 \\
\hline 4009.75 & 8.473 & 3998.67 & 8.373 & 3998.68 & 8.473 & 4000.15 & 8.433 \\
\hline 4009.75 & 8.453 & 3998.67 & 8.433 & 3998.68 & 8.473 & 4000.15 & 8.453 \\
\hline 4009.75 & 8.413 & 3998.67 & 8.413 & 3998.68 & 8.513 & 4000.15 & 8.393 \\
\hline 4009.75 & 8.453 & 3998.67 & 8.413 & 3998.68 & 8.453 & 4000.15 & 8.413 \\
\hline 4009.75 & 8.433 & 3998.67 & 8.393 & 3998.68 & 8.513 & 4000.15 & 8.453 \\
\hline 4009.75 & 8.493 & 3998.67 & 8.393 & 3998.68 & 8.513 & 4000.15 & 8.473 \\
\hline 4009.75 & 8.453 & 3998.67 & 8.413 & 3998.68 & 8.473 & 4000.15 & 8.473 \\
\hline
\end{tabular}


Table A.1 Data Set1 - Relative Distance Calibration: $0 \mathrm{~m}$ to $60 \mathrm{~m}$

\begin{tabular}{|c|c|c|c|c|c|c|c|}
\hline \multicolumn{8}{|c|}{$\begin{array}{l}\text { Location: Environmentally controlled } \\
\text { No Filter } \\
\text { Test dates: July 14-15, } 1999\end{array}$} \\
\hline $\begin{array}{l}\text { Interfero. } \\
\text { Dist. } \\
(\mathrm{mm})\end{array}$ & $\begin{array}{l}\text { Blck, not } \\
\text { shiny } \\
\text { (m) }\end{array}$ & $\begin{array}{l}\text { Interfero. } \\
\text { Dist. } \\
(\mathrm{mm})\end{array}$ & $\begin{array}{c}\text { Blck, shiny } \\
\text { (m) }\end{array}$ & $\begin{array}{l}\text { Interfero. } \\
\text { Dist. } \\
(\mathrm{mm})\end{array}$ & $\begin{array}{l}\text { Dk. Grey, } \\
\text { rough } \\
\text { (m) }\end{array}$ & $\begin{array}{l}\text { Interfero. } \\
\text { Dist. } \\
(\mathrm{mm})\end{array}$ & $\begin{array}{l}\text { Pink, not } \\
\text { shiny } \\
\text { (m) }\end{array}$ \\
\hline 4009.75 & 8.433 & 3998.67 & 8.373 & 3998.68 & 8.473 & 4000.15 & 8.453 \\
\hline 4009.75 & 8.433 & 3998.67 & 8.373 & 3998.68 & 8.453 & 4000.15 & 8.473 \\
\hline 4009.75 & 8.433 & 3998.67 & 8.433 & 3998.68 & 8.453 & 4000.15 & 8.393 \\
\hline 4009.75 & 8.453 & 3998.67 & 8.373 & 3998.68 & 8.453 & 4000.15 & 8.433 \\
\hline 4009.75 & 8.493 & 3998.67 & 8.413 & 3998.68 & 8.453 & 4000.15 & 8.433 \\
\hline 4009.75 & 8.433 & 3998.67 & 8.393 & 3998.68 & 8.433 & 4000.15 & 8.413 \\
\hline 4009.75 & 8.433 & 3998.67 & 8.393 & 3998.68 & 8.493 & 4000.15 & 8.453 \\
\hline 4009.75 & 8.453 & 3998.67 & 8.433 & 3998.68 & 8.553 & 4000.15 & 8.433 \\
\hline 4009.75 & 8.433 & 3998.67 & 8.433 & 3998.68 & 8.473 & 4000.15 & 8.413 \\
\hline 4009.75 & 8.433 & 3998.67 & 8.433 & 3998.68 & 8.413 & 4000.15 & 8.433 \\
\hline 4009.75 & 8.433 & 3998.67 & 8.373 & 3998.68 & 8.433 & 4000.15 & 8.413 \\
\hline 4009.75 & 8.433 & 3998.67 & 8.433 & 3998.68 & 8.493 & 4000.15 & 8.393 \\
\hline 4009.75 & 8.473 & 3998.67 & 8.433 & 3998.68 & 8.493 & 4000.15 & 8.473 \\
\hline 4009.75 & 8.433 & 3998.67 & 8.413 & 3998.68 & 8.493 & 4000.15 & 8.433 \\
\hline 4009.75 & 8.453 & 3998.67 & 8.373 & 3998.68 & 8.453 & 4000.15 & 8.433 \\
\hline 4009.75 & 8.453 & 3998.67 & 8.433 & 3998.68 & 8.433 & 4000.15 & 8.433 \\
\hline 4009.75 & 8.453 & 3998.67 & 8.413 & 3998.68 & 8.453 & 4000.15 & 8.433 \\
\hline 4009.75 & 8.473 & 3998.67 & 8.393 & 3998.68 & 8.453 & 4000.15 & 8.453 \\
\hline 6001.70 & 10.456 & 6000.67 & 10.416 & 5999.74 & 10.496 & 6001.67 & 10.416 \\
\hline 6001.70 & 10.416 & 6000.67 & 10.356 & 5999.74 & 10.456 & 6001.67 & 10.436 \\
\hline 6001.70 & 10.416 & 6000.67 & 10.376 & 5999.74 & 10.416 & 6001.67 & 10.456 \\
\hline 6001.70 & 10.396 & 6000.67 & 10.436 & 5999.74 & 10.476 & 6001.67 & 10.396 \\
\hline 6001.70 & 10.436 & 6000.67 & 10.396 & 5999.74 & 10.396 & 6001.67 & 10.356 \\
\hline 6001.70 & 10.396 & 6000.67 & 10.396 & 5999.74 & 10.476 & 6001.67 & 10.496 \\
\hline 6001.70 & 10.416 & 6000.67 & 10.376 & 5999.74 & 10.416 & 6001.67 & 10.396 \\
\hline 6001.70 & 10.476 & 6000.67 & 10.396 & 5999.74 & 10.456 & 6001.67 & 10.436 \\
\hline 6001.70 & 10.436 & 6000.67 & 10.416 & 5999.74 & 10.496 & 6001.67 & 10.476 \\
\hline 6001.70 & 10.416 & 6000.67 & 10.396 & 5999.74 & 10.456 & 6001.67 & 10.416 \\
\hline 6001.70 & 10.476 & 6000.67 & 10.416 & 5999.74 & 10.436 & 6001.67 & 10.456 \\
\hline 6001.70 & 10.456 & 6000.67 & 10.416 & 5999.74 & 10.456 & 6001.67 & 10.456 \\
\hline 6001.70 & 10.416 & 6000.67 & 10.396 & 5999.74 & 10.476 & 6001.67 & 10.456 \\
\hline 6001.70 & 10.416 & 6000.67 & 10.416 & 5999.74 & 10.476 & 6001.67 & 10.416 \\
\hline 6001.70 & 10.456 & 6000.67 & 10.416 & 5999.74 & 10.436 & 6001.67 & 10.396 \\
\hline 6001.70 & 10.416 & 6000.67 & 10.356 & 5999.74 & 10.456 & 6001.67 & 10.456 \\
\hline 6001.70 & 10.476 & 6000.67 & 10.396 & 5999.74 & 10.496 & 6001.67 & 10.456 \\
\hline 6001.70 & 10.456 & 6000.67 & 10.436 & 5999.74 & 10.416 & 6001.67 & 10.436 \\
\hline 6001.70 & 10.416 & 6000.67 & 10.376 & 5999.74 & 10.496 & 6001.67 & 10.436 \\
\hline 6001.70 & 10.376 & 6000.67 & 10.396 & 5999.74 & 10.476 & 6001.67 & 10.436 \\
\hline 6001.70 & 10.456 & 6000.67 & 10.396 & 5999.74 & 10.456 & 6001.67 & 10.436 \\
\hline 6001.70 & 10.436 & 6000.67 & 10.456 & 5999.74 & 10.476 & 6001.67 & 10.436 \\
\hline 6001.70 & 10.436 & 6000.67 & 10.416 & 5999.74 & 10.456 & 6001.67 & 10.476 \\
\hline 6001.70 & 10.456 & 6000.67 & 10.396 & 5999.74 & 10.436 & 6001.67 & 10.436 \\
\hline 6001.70 & 10.456 & 6000.67 & 10.416 & 5999.74 & 10.456 & 6001.67 & 10.456 \\
\hline 7999.24 & 12.439 & 7998.05 & 12.399 & 7998.77 & 12.439 & 8000.42 & 12.439 \\
\hline 7999.24 & 12.459 & 7998.05 & 12.399 & 7998.77 & 12.459 & 8000.42 & 12.459 \\
\hline 7999.24 & 12.419 & 7998.05 & 12.399 & 7998.77 & 12.500 & 8000.42 & 12.479 \\
\hline 7999.24 & 12.459 & 7998.05 & 12.419 & 7998.77 & 12.500 & 8000.42 & 12.439 \\
\hline 7999.24 & 12.459 & 7998.05 & 12.399 & 7998.77 & 12.479 & 8000.42 & 12.439 \\
\hline 7999.24 & 12.479 & 7998.05 & 12.419 & 7998.77 & 12.439 & 8000.42 & 12.399 \\
\hline 7999.24 & 12.459 & 7998.05 & 12.419 & 7998.77 & 12.439 & 8000.42 & 12.439 \\
\hline 7999.24 & 12.479 & 7998.05 & 12.419 & 7998.77 & 12.500 & 8000.42 & 12.479 \\
\hline 7999.24 & 12.439 & 7998.05 & 12.419 & 7998.77 & 12.500 & 8000.42 & 12.439 \\
\hline 7999.24 & 12.479 & 7998.05 & 12.399 & 7998.77 & 12.439 & 8000.42 & 12.479 \\
\hline 7999.24 & 12.439 & 7998.05 & 12.439 & 7998.77 & 12.459 & 8000.42 & 12.419 \\
\hline 7999.24 & 12.459 & 7998.05 & 12.439 & 7998.77 & 12.439 & 8000.42 & 12.439 \\
\hline 7999.24 & 12.459 & 7998.05 & 12.419 & 7998.77 & 12.479 & 8000.42 & 12.459 \\
\hline 7999.24 & 12.439 & 7998.05 & 12.399 & 7998.77 & 12.479 & 8000.42 & 12.419 \\
\hline 7999.24 & 12.459 & 7998.05 & 12.379 & 7998.77 & 12.439 & 8000.42 & 12.459 \\
\hline 7999.24 & 12.419 & 7998.05 & 12.419 & 7998.77 & 12.459 & 8000.42 & 12.459 \\
\hline 7999.24 & 12.439 & 7998.05 & 12.379 & 7998.77 & 12.479 & 8000.42 & 12.419 \\
\hline
\end{tabular}


Table A.1 Data Set1 - Relative Distance Calibration: $0 \mathrm{~m}$ to $60 \mathrm{~m}$

\begin{tabular}{|c|c|c|c|c|c|c|c|}
\hline \multicolumn{8}{|c|}{$\begin{array}{l}\text { Location: Environmentally controlled } \\
\text { No Filter } \\
\text { Test dates: July 14-15, } 1999\end{array}$} \\
\hline $\begin{array}{l}\text { Interfero. } \\
\text { Dist. } \\
(\mathrm{mm})\end{array}$ & $\begin{array}{l}\text { Blck, not } \\
\text { shiny } \\
\text { (m) }\end{array}$ & $\begin{array}{l}\text { Interfero. } \\
\text { Dist. } \\
(\mathrm{mm})\end{array}$ & $\begin{array}{l}\text { Blck, shiny } \\
\text { (m) }\end{array}$ & $\begin{array}{l}\text { Interfero. } \\
\text { Dist. } \\
(\mathrm{mm})\end{array}$ & $\begin{array}{l}\text { Dk. Grey, } \\
\text { rough } \\
\text { (m) }\end{array}$ & $\begin{array}{l}\text { Interfero. } \\
\text { Dist. } \\
(\mathrm{mm})\end{array}$ & $\begin{array}{l}\text { Pink, not } \\
\text { shiny } \\
\text { (m) }\end{array}$ \\
\hline 7999.24 & 12.459 & 7998.05 & 12.399 & 7998.77 & 12.439 & 8000.42 & 12.439 \\
\hline 7999.24 & 12.459 & 7998.05 & 12.399 & 7998.77 & 12.439 & 8000.42 & 12.459 \\
\hline 7999.24 & 12.459 & 7998.05 & 12.399 & 7998.77 & 12.479 & 8000.42 & 12.419 \\
\hline 7999.24 & 12.439 & 7998.05 & 12.419 & 7998.77 & 12.459 & 8000.42 & 12.399 \\
\hline 7999.24 & 12.459 & 7998.05 & 12.399 & 7998.77 & 12.459 & 8000.42 & 12.439 \\
\hline 7999.24 & 12.459 & 7998.05 & 12.379 & 7998.77 & 12.419 & 8000.42 & 12.479 \\
\hline 7999.24 & 12.500 & 7998.05 & 12.439 & 7998.77 & 12.459 & 8000.42 & 12.419 \\
\hline 7999.24 & 12.459 & 7998.05 & 12.419 & 7998.77 & 12.479 & 8000.42 & 12.439 \\
\hline 10000.69 & 14.443 & 9999.7 & 14.363 & 9999.81 & 14.463 & 10000.91 & 14.463 \\
\hline 10000.69 & 14.423 & 9999.7 & 14.383 & 9999.81 & 14.463 & 10000.91 & 14.443 \\
\hline 10000.69 & 14.483 & 9999.7 & 14.363 & 9999.81 & 14.504 & 10000.91 & 14.463 \\
\hline 10000.69 & 14.443 & 9999.7 & 14.423 & 9999.81 & 14.403 & 10000.91 & 14.403 \\
\hline 10000.69 & 14.463 & 9999.7 & 14.383 & 9999.81 & 14.483 & 10000.91 & 14.443 \\
\hline 10000.69 & 14.423 & 9999.7 & 14.363 & 9999.81 & 14.463 & 10000.91 & 14.463 \\
\hline 10000.69 & 14.443 & 9999.7 & 14.383 & 9999.81 & 14.504 & 10000.91 & 14.483 \\
\hline 10000.69 & 14.443 & 9999.7 & 14.383 & 9999.81 & 14.463 & 10000.91 & 14.443 \\
\hline 10000.69 & 14.403 & 9999.7 & 14.383 & 9999.81 & 14.483 & 10000.91 & 14.423 \\
\hline 10000.69 & 14.443 & 9999.7 & 14.403 & 9999.81 & 14.423 & 10000.91 & 14.463 \\
\hline 10000.69 & 14.443 & 9999.7 & 14.403 & 9999.81 & 14.483 & 10000.91 & 14.423 \\
\hline 10000.69 & 14.423 & 9999.7 & 14.383 & 9999.81 & 14.463 & 10000.91 & 14.423 \\
\hline 10000.69 & 14.443 & 9999.7 & 14.423 & 9999.81 & 14.443 & 10000.91 & 14.443 \\
\hline 10000.69 & 14.463 & 9999.7 & 14.383 & 9999.81 & 14.483 & 10000.91 & 14.483 \\
\hline 10000.69 & 14.423 & 9999.7 & 14.403 & 9999.81 & 14.483 & 10000.91 & 14.483 \\
\hline 10000.69 & 14.443 & 9999.7 & 14.403 & 9999.81 & 14.499 & 10000.91 & 14.403 \\
\hline 10000.69 & 14.443 & 9999.7 & 14.363 & 9999.81 & 14.483 & 10000.91 & 14.463 \\
\hline 10000.69 & 14.463 & 9999.7 & 14.363 & 9999.81 & 14.463 & 10000.91 & 14.463 \\
\hline 10000.69 & 14.443 & 9999.7 & 14.403 & 9999.81 & 14.483 & 10000.91 & 14.463 \\
\hline 10000.69 & 14.463 & 9999.7 & 14.423 & 9999.81 & 14.463 & 10000.91 & 14.483 \\
\hline 10000.69 & 14.443 & 9999.7 & 14.383 & 9999.81 & 14.443 & 10000.91 & 14.463 \\
\hline 10000.69 & 14.443 & 9999.7 & 14.423 & 9999.81 & 14.463 & 10000.91 & 14.443 \\
\hline 10000.69 & 14.443 & 9999.7 & 14.403 & 9999.81 & 14.483 & 10000.91 & 14.423 \\
\hline 10000.69 & 14.483 & 9999.7 & 14.403 & 9999.81 & 14.443 & 10000.91 & 14.483 \\
\hline 10000.69 & 14.443 & 9999.7 & 14.363 & 9999.81 & 14.483 & 10000.91 & 14.443 \\
\hline 14996.22 & 19.425 & 14995.35 & 19.405 & 14996.92 & 19.505 & 14996.90 & 19.465 \\
\hline 14996.22 & 19.445 & 14995.35 & 19.405 & 14996.92 & 19.425 & 14996.90 & 19.385 \\
\hline 14996.22 & 19.405 & 14995.35 & 19.405 & 14996.92 & 19.465 & 14996.90 & 19.445 \\
\hline 14996.22 & 19.445 & 14995.35 & 19.425 & 14996.92 & 19.485 & 14996.90 & 19.445 \\
\hline 14996.22 & 19.425 & 14995.35 & 19.445 & 14996.92 & 19.385 & 14996.90 & 19.445 \\
\hline 14996.22 & 19.385 & 14995.35 & 19.405 & 14996.92 & 19.485 & 14996.90 & 19.445 \\
\hline 14996.22 & 19.445 & 14995.35 & 19.405 & 14996.92 & 19.485 & 14996.90 & 19.425 \\
\hline 14996.22 & 19.445 & 14995.35 & 19.425 & 14996.92 & 19.445 & 14996.90 & 19.425 \\
\hline 14996.22 & 19.465 & 14995.35 & 19.385 & 14996.92 & 19.425 & 14996.90 & 19.425 \\
\hline 14996.22 & 19.445 & 14995.35 & 19.385 & 14996.92 & 19.505 & 14996.90 & 19.485 \\
\hline 14996.22 & 19.425 & 14995.35 & 19.405 & 14996.92 & 19.445 & 14996.90 & 19.445 \\
\hline 14996.22 & 19.445 & 14995.35 & 19.425 & 14996.92 & 19.445 & 14996.90 & 19.425 \\
\hline 14996.22 & 19.425 & 14995.35 & 19.445 & 14996.92 & 19.525 & 14996.90 & 19.445 \\
\hline 14996.22 & 19.445 & 14995.35 & 19.385 & 14996.92 & 19.485 & 14996.90 & 19.485 \\
\hline 14996.22 & 19.485 & 14995.35 & 19.405 & 14996.92 & 19.445 & 14996.90 & 19.485 \\
\hline 14996.22 & 19.425 & 14995.35 & 19.445 & 14996.92 & 19.505 & 14996.90 & 19.445 \\
\hline 14996.22 & 19.405 & 14995.35 & 19.425 & 14996.92 & 19.465 & 14996.90 & 19.425 \\
\hline 14996.22 & 19.465 & 14995.35 & 19.405 & 14996.92 & 19.485 & 14996.90 & 19.465 \\
\hline 14996.22 & 19.425 & 14995.35 & 19.405 & 14996.92 & 19.445 & 14996.90 & 19.385 \\
\hline 14996.22 & 19.465 & 14995.35 & 19.405 & 14996.92 & 19.445 & 14996.90 & 19.425 \\
\hline 14996.22 & 19.465 & 14995.35 & 19.405 & 14996.92 & 19.485 & 14996.90 & 19.385 \\
\hline 14996.22 & 19.405 & 14995.35 & 19.385 & 14996.92 & 19.505 & 14996.90 & 19.425 \\
\hline 14996.22 & 19.465 & 14995.35 & 19.405 & 14996.92 & 19.445 & 14996.90 & 19.445 \\
\hline 14996.22 & 19.465 & 14995.35 & 19.465 & 14996.92 & 19.465 & 14996.90 & 19.445 \\
\hline 14996.22 & 19.465 & 14995.35 & 19.405 & 14996.92 & 19.465 & 14996.90 & 19.425 \\
\hline 20009.44 & 24.525 & 20011.32 & 24.465 & 20010.28 & 24.545 & 20011.23 & 24.465 \\
\hline 20009.44 & 24.465 & 20011.32 & 24.445 & 20010.28 & 24.505 & 20011.23 & 24.465 \\
\hline
\end{tabular}


Table A.1 Data Set1 - Relative Distance Calibration: $0 \mathrm{~m}$ to $60 \mathrm{~m}$

\begin{tabular}{|c|c|c|c|c|c|c|c|}
\hline $\begin{array}{l}\text { Location: E } \\
\text { No Filter } \\
\text { Test dates: }\end{array}$ & $\begin{array}{l}\text { vironment } \\
\text { ly } 14-15 \text {, }\end{array}$ & controllec & b, Bldg. 220 & & & File: re & list_anal.xls \\
\hline $\begin{array}{c}\text { Interfero. } \\
\text { Dist. } \\
(\mathrm{mm})\end{array}$ & $\begin{array}{c}\text { Blck, not } \\
\text { shiny } \\
\text { (m) }\end{array}$ & $\begin{array}{c}\text { Interfero. } \\
\text { Dist. } \\
(\mathrm{mm})\end{array}$ & $\begin{array}{l}\text { Blck, shiny } \\
\text { (m) }\end{array}$ & $\begin{array}{c}\text { Interfero. } \\
\text { Dist. } \\
(\mathrm{mm})\end{array}$ & $\begin{array}{l}\text { Dk. Grey, } \\
\text { rough } \\
\text { (m) }\end{array}$ & $\begin{array}{c}\text { Interfero. } \\
\text { Dist. } \\
(\mathrm{mm})\end{array}$ & $\begin{array}{l}\text { Pink, not } \\
\text { shiny } \\
\text { (m) }\end{array}$ \\
\hline 20009.44 & 24.425 & 20011.32 & 24.445 & 20010.28 & 24.545 & 20011.23 & 24.445 \\
\hline 20009.44 & 24.485 & 20011.32 & 24.485 & 20010.28 & 24.505 & 20011.23 & 24.445 \\
\hline 20009.44 & 24.485 & 20011.32 & 24.485 & 20010.28 & 24.565 & 20011.23 & 24.445 \\
\hline 20009.44 & 24.445 & 20011.32 & 24.465 & 20010.28 & 24.525 & 20011.23 & 24.465 \\
\hline 20009.44 & 24.425 & 20011.32 & 24.425 & 20010.28 & 24.565 & 20011.23 & 24.445 \\
\hline 20009.44 & 24.485 & 20011.32 & 24.425 & 20010.28 & 24.525 & 20011.23 & 24.465 \\
\hline 20009.44 & 24.465 & 20011.32 & 24.465 & 20010.28 & 24.485 & 20011.23 & 24.465 \\
\hline 20009.44 & 24.505 & 20011.32 & 24.445 & 20010.28 & 24.585 & 20011.23 & 24.425 \\
\hline 20009.44 & 24.485 & 20011.32 & 24.465 & 20010.28 & 24.505 & 20011.23 & 24.445 \\
\hline 20009.44 & 24.425 & 20011.32 & 24.465 & 20010.28 & 24.425 & 20011.23 & 24.425 \\
\hline 20009.44 & 24.445 & 20011.32 & 24.465 & 20010.28 & 24.525 & 20011.23 & 24.445 \\
\hline 20009.44 & 24.465 & 20011.32 & 24.405 & 20010.28 & 24.505 & 20011.23 & 24.445 \\
\hline 20009.44 & 24.445 & 20011.32 & 24.425 & 20010.28 & 24.445 & 20011.23 & 24.465 \\
\hline 20009.44 & 24.445 & 20011.32 & 24.405 & 20010.28 & 24.545 & 20011.23 & 24.445 \\
\hline 20009.44 & 24.505 & 20011.32 & 24.425 & 20010.28 & 24.545 & 20011.23 & 24.405 \\
\hline 20009.44 & 24.485 & 20011.32 & 24.465 & 20010.28 & 24.545 & 20011.23 & 24.445 \\
\hline 20009.44 & 24.465 & 20011.32 & 24.465 & 20010.28 & 24.545 & 20011.23 & 24.465 \\
\hline 20009.44 & 24.445 & 20011.32 & 24.425 & 20010.28 & 24.485 & 20011.23 & 24.465 \\
\hline 20009.44 & 24.465 & 20011.32 & 24.485 & 20010.28 & 24.545 & 20011.23 & 24.465 \\
\hline 20009.44 & 24.425 & 20011.32 & 24.465 & 20010.28 & 24.485 & 20011.23 & 24.480 \\
\hline 20009.44 & 24.445 & 20011.32 & 24.405 & 20010.28 & 24.485 & 20011.23 & 24.445 \\
\hline 20009.44 & 24.465 & 20011.32 & 24.485 & 20010.28 & 24.505 & 20011.23 & 24.465 \\
\hline 20009.44 & 24.465 & 20011.32 & 24.445 & 20010.28 & 24.485 & 20011.23 & 24.485 \\
\hline 30003.46 & 34.405 & 30003.76 & 34.485 & 30005.96 & 34.525 & 30007.11 & 34.465 \\
\hline 30003.46 & 34.565 & 30003.76 & 34.545 & 30005.96 & 34.705 & 30007.11 & 34.425 \\
\hline 30003.46 & 34.545 & 30003.76 & 34.525 & 30005.96 & 34.625 & 30007.11 & 34.485 \\
\hline 30003.46 & 34.545 & 30003.76 & 34.465 & 30005.96 & 34.625 & 30007.11 & 34.425 \\
\hline 30003.46 & 34.505 & 30003.76 & 34.545 & 30005.96 & 34.585 & 30007.11 & 34.485 \\
\hline 30003.46 & 34.525 & 30003.76 & 34.465 & 30005.96 & 34.545 & 30007.11 & 34.425 \\
\hline 30003.46 & 34.565 & 30003.76 & 34.485 & 30005.96 & 34.505 & 30007.11 & 34.425 \\
\hline 30003.46 & 34.525 & 30003.76 & 34.505 & 30005.96 & 34.605 & 30007.11 & 34.425 \\
\hline 30003.46 & 34.525 & 30003.76 & 34.445 & 30005.96 & 34.645 & 30007.11 & 34.445 \\
\hline 30003.46 & 34.000 & 30003.76 & 34.505 & 30005.96 & 34.605 & 30007.11 & 34.465 \\
\hline 30003.46 & 34.545 & 30003.76 & 34.485 & 30005.96 & 34.525 & 30007.11 & 34.445 \\
\hline 30003.46 & 34.465 & 30003.76 & 34.485 & 30005.96 & 34.585 & 30007.11 & 34.385 \\
\hline 30003.46 & 34.525 & 30003.76 & 34.505 & 30005.96 & 34.585 & 30007.11 & 34.485 \\
\hline 30003.46 & 34.465 & 30003.76 & 34.505 & 30005.96 & 34.525 & 30007.11 & 34.425 \\
\hline 30003.46 & 34.485 & 30003.76 & 34.505 & 30005.96 & 34.545 & 30007.11 & 34.465 \\
\hline 30003.46 & 34.545 & 30003.76 & 34.485 & 30005.96 & 34.525 & 30007.11 & 34.485 \\
\hline 30003.46 & 34.505 & 30003.76 & 34.505 & 30005.96 & 34.505 & 30007.11 & 34.465 \\
\hline 30003.46 & 34.585 & 30003.76 & 34.485 & 30005.96 & 34.625 & 30007.11 & 34.425 \\
\hline 30003.46 & 34.465 & 30003.76 & 34.465 & 30005.96 & 34.605 & 30007.11 & 34.485 \\
\hline 30003.46 & 34.545 & 30003.76 & 34.505 & 30005.96 & 34.645 & 30007.11 & 34.425 \\
\hline 30003.46 & 34.625 & 30003.76 & 34.445 & 30005.96 & 34.485 & 30007.11 & 34.445 \\
\hline 30003.46 & 34.545 & 30003.76 & 34.485 & 30005.96 & 34.565 & 30007.11 & 34.465 \\
\hline 30003.46 & 34.445 & 30003.76 & 34.485 & 30005.96 & 34.565 & 30007.11 & 34.445 \\
\hline 30003.46 & 34.585 & 30003.76 & 34.485 & 30005.96 & 34.505 & 30007.11 & 34.420 \\
\hline 30003.46 & 34.445 & 30003.76 & 34.405 & 30005.96 & 34.465 & 30007.11 & 34.405 \\
\hline 39961.74 & 44.405 & 39963.03 & 44.485 & 39963.32 & 44.485 & 39965.70 & 44.425 \\
\hline 39961.74 & 44.505 & 39963.03 & 44.465 & 39963.32 & 44.405 & 39965.70 & 44.405 \\
\hline 39961.74 & 44.505 & 39963.03 & 44.545 & 39963.32 & 44.465 & 39965.70 & 44.425 \\
\hline 39961.74 & 44.505 & 39963.03 & 44.445 & 39963.32 & 44.525 & 39965.70 & 44.405 \\
\hline 39961.74 & 44.545 & 39963.03 & 44.465 & 39963.32 & 44.445 & 39965.70 & 44.445 \\
\hline 39961.74 & 44.465 & 39963.03 & 44.485 & 39963.32 & 44.485 & 39965.70 & 44.425 \\
\hline 39961.74 & 44.545 & 39963.03 & 44.405 & 39963.32 & 44.485 & 39965.70 & 44.425 \\
\hline 39961.74 & 44.465 & 39963.03 & 44.465 & 39963.32 & 44.585 & 39965.70 & 44.365 \\
\hline 39961.74 & 44.525 & 39963.03 & 44.465 & 39963.32 & 44.605 & 39965.70 & 44.385 \\
\hline 39961.74 & 44.565 & 39963.03 & 44.465 & 39963.32 & 44.445 & 39965.70 & 44.445 \\
\hline 39961.74 & 44.405 & 39963.03 & 44.465 & 39963.32 & 44.585 & 39965.70 & 44.405 \\
\hline 39961.74 & 44.485 & 39963.03 & 44.485 & 39963.32 & 44.445 & 39965.70 & 44.385 \\
\hline
\end{tabular}


Table A.1 Data Set1 - Relative Distance Calibration: $0 \mathrm{~m}$ to $60 \mathrm{~m}$

\begin{tabular}{|c|c|c|c|c|c|c|c|}
\hline \multicolumn{8}{|c|}{$\begin{array}{l}\text { Location: Environmentally controlled lab, Bldg. } 220 \quad \text { File: rel_dist_anal.xls } \\
\text { No Filter } \\
\text { Test dates: July } 14-15,1999\end{array}$} \\
\hline $\begin{array}{l}\text { Interfero. } \\
\text { Dist. } \\
(\mathrm{mm})\end{array}$ & $\begin{array}{l}\text { Blck, not } \\
\text { shiny } \\
\text { (m) }\end{array}$ & $\begin{array}{l}\text { Interfero. } \\
\text { Dist. } \\
(\mathrm{mm})\end{array}$ & $\begin{array}{l}\text { Blck, shiny } \\
\text { (m) }\end{array}$ & $\begin{array}{l}\text { Interfero. } \\
\text { Dist. } \\
(\mathrm{mm})\end{array}$ & $\begin{array}{l}\text { Dk. Grey, } \\
\text { rough } \\
(\mathrm{m})\end{array}$ & $\begin{array}{c}\text { Interfero. } \\
\text { Dist. } \\
(\mathrm{mm})\end{array}$ & $\begin{array}{l}\text { Pink, not } \\
\text { shiny } \\
\text { (m) }\end{array}$ \\
\hline 39961.74 & 44.505 & 39963.03 & 44.445 & 39963.32 & 44.565 & 39965.70 & 44.405 \\
\hline 39961.74 & 44.605 & 39963.03 & 44.485 & 39963.32 & 44.525 & 39965.70 & 44.405 \\
\hline 39961.74 & 44.545 & 39963.03 & 44.505 & 39963.32 & 44.525 & 39965.70 & 44.385 \\
\hline 39961.74 & 44.545 & 39963.03 & 44.485 & 39963.32 & 44.465 & 39965.70 & 44.425 \\
\hline 39961.74 & 44.605 & 39963.03 & 44.465 & 39963.32 & 44.525 & 39965.70 & 44.445 \\
\hline 39961.74 & 44.605 & 39963.03 & 44.485 & 39963.32 & 44.505 & 39965.70 & 44.405 \\
\hline 39961.74 & 44.485 & 39963.03 & 44.465 & 39963.32 & 44.405 & 39965.70 & 44.445 \\
\hline 39961.74 & 44.445 & 39963.03 & 44.485 & 39963.32 & 44.425 & 39965.70 & 44.385 \\
\hline 39961.74 & 44.605 & 39963.03 & 44.365 & 39963.32 & 44.445 & 39965.70 & 44.385 \\
\hline 39961.74 & 44.505 & 39963.03 & 44.445 & 39963.32 & 44.665 & 39965.70 & 44.405 \\
\hline 39961.74 & 44.505 & 39963.03 & 44.405 & 39963.32 & 44.605 & 39965.70 & 44.385 \\
\hline 39961.74 & 44.485 & 39963.03 & 44.445 & 39963.32 & 44.445 & 39965.70 & 44.385 \\
\hline 39961.74 & 44.505 & 39963.03 & 44.425 & 39963.32 & 44.645 & 39965.70 & 44.425 \\
\hline & & 49914.07 & 54.565 & & & 49926.43 & 54.465 \\
\hline & & 49914.07 & 54.465 & & & 49926.43 & 54.465 \\
\hline & & 49914.07 & 54.465 & & & 49926.43 & 54.445 \\
\hline & & 49914.07 & 54.585 & & & 49926.43 & 54.385 \\
\hline & & 49914.07 & 54.525 & & & 49926.43 & 54.405 \\
\hline & & 49914.07 & 54.405 & & & 49926.43 & 54.445 \\
\hline & & 49914.07 & 54.545 & & & 49926.43 & 54.445 \\
\hline & & 49914.07 & 54.425 & & & 49926.43 & 54.465 \\
\hline & & 49914.07 & 54.565 & & & 49926.43 & 54.445 \\
\hline & & 49914.07 & 54.405 & & & 49926.43 & 54.385 \\
\hline & & 49914.07 & 54.485 & & & 49926.43 & 54.425 \\
\hline & & 49914.07 & 54.465 & & & 49926.43 & 54.465 \\
\hline & & 49914.07 & 54.445 & & & 49926.43 & 54.485 \\
\hline & & 49914.07 & 54.485 & & & 49926.43 & 54.465 \\
\hline & & 49914.07 & 54.485 & & & 49926.43 & 54.405 \\
\hline & & 49914.07 & 54.485 & & & 49926.43 & 54.405 \\
\hline & & 49914.07 & 54.525 & & & 49926.43 & 54.405 \\
\hline & & 49914.07 & 54.565 & & & 49926.43 & 54.445 \\
\hline & & 49914.07 & 54.585 & & & 49926.43 & 54.385 \\
\hline & & 49914.07 & 54.385 & & & 49926.43 & 54.485 \\
\hline & & 49914.07 & 54.345 & & & 49926.43 & 54.445 \\
\hline & & 49914.07 & 54.445 & & & 49926.43 & 54.465 \\
\hline & & 49914.07 & 54.365 & & & 49926.43 & 54.465 \\
\hline & & 49914.07 & 54.485 & & & 49926.43 & 54.445 \\
\hline & & 49914.07 & 54.405 & & & 49926.43 & 54.425 \\
\hline
\end{tabular}


Table A.1 Data Set 1 - Relative Distance Calibration: $0 \mathrm{~m}$ to $60 \mathrm{~m}$

Location: Environmentally controlled lab, Bldg. 220

No Filter

File: rel_dist_anal.xls

Test dates: July 14-15, 1999

Location: Environmentally controlled

lab, Bldg. 220

No Filter

File: 8_22_99_laser.xls

Test date: Aug. 2, 1999

\begin{tabular}{|c|c|c|c|c|c|}
\hline $\begin{array}{l}\text { Interfero. Dist. } \\
(\mathrm{mm})\end{array}$ & $\begin{array}{l}\text { Yllw, not shiny } \\
\text { (m) }\end{array}$ & $\begin{array}{l}\text { Interfero. Dist. } \\
(\mathrm{mm})\end{array}$ & $\begin{array}{l}\text { Grn, not shiny } \\
\text { (m) }\end{array}$ & $\begin{array}{l}\text { Interfero. Dist. } \\
(\mathrm{mm})\end{array}$ & $\begin{array}{l}\text { Grn, not shiny - } \\
\text { recal. } \\
\text { (m) }\end{array}$ \\
\hline 0 & 4.463 & 0.00 & 4.448 & 0.00 & 4.451 \\
\hline 0 & 4.431 & 0.00 & 4.468 & 0.00 & 4.447 \\
\hline 0 & 4.407 & 0.00 & 4.448 & 0.00 & 4.431 \\
\hline 0 & 4.427 & 0.00 & 4.468 & 0.00 & 4.451 \\
\hline 0 & 4.427 & 0.00 & 4.448 & 0.00 & 4.447 \\
\hline 0 & 4.427 & 0.00 & 4.468 & 0.00 & 4.427 \\
\hline 0 & 4.407 & 0.00 & 4.468 & 0.00 & 4.411 \\
\hline 0 & 4.427 & 0.00 & 4.428 & 0.00 & 4.467 \\
\hline 0 & 4.447 & 0.00 & 4.468 & 0.00 & 4.431 \\
\hline 0 & 4.447 & 0.00 & 4.468 & 0.00 & 4.447 \\
\hline 0 & 4.387 & 0.00 & 4.448 & 0.00 & 4.447 \\
\hline 0 & 4.427 & 0.00 & 4.448 & 0.00 & 4.427 \\
\hline 0 & 4.427 & 0.00 & 4.468 & 0.00 & 4.447 \\
\hline 0 & 4.427 & 0.00 & 4.448 & 0.00 & 4.447 \\
\hline 0 & 4.427 & 0.00 & 4.448 & 0.00 & 4.447 \\
\hline 0 & 4.427 & 0.00 & 4.448 & 0.00 & 4.427 \\
\hline 0 & 4.407 & 0.00 & 4.468 & 0.00 & 4.467 \\
\hline 0 & 4.407 & 0.00 & 4.468 & 0.00 & 4.427 \\
\hline 0 & 4.407 & 0.00 & 4.428 & 0.00 & 4.427 \\
\hline 0 & 4.427 & 0.00 & 4.448 & 0.00 & 4.427 \\
\hline 0 & 4.407 & 0.00 & 4.448 & 0.00 & 4.447 \\
\hline 0 & 4.407 & 0.00 & 4.468 & 0.00 & 4.447 \\
\hline 0 & 4.387 & 0.00 & 4.428 & 0.00 & 4.447 \\
\hline 0 & 4.407 & 0.00 & 4.448 & 0.00 & 4.467 \\
\hline 0 & 4.407 & 0.00 & 4.448 & 2000.58 & 6.433 \\
\hline 2001.73 & 6.469 & 2000.88 & 6.450 & 2000.58 & 6.453 \\
\hline 2001.73 & 6.449 & 2000.88 & 6.450 & 2000.58 & 6.453 \\
\hline 2001.73 & 6.449 & 2000.88 & 6.470 & 2000.58 & 6.473 \\
\hline 2001.73 & 6.389 & 2000.88 & 6.450 & 2000.58 & 6.453 \\
\hline 2001.73 & 6.409 & 2000.88 & 6.490 & 2000.58 & 6.493 \\
\hline 2001.73 & 6.449 & 2000.88 & 6.450 & 2000.58 & 6.453 \\
\hline 2001.73 & 6.409 & 2000.88 & 6.490 & 2000.58 & 6.473 \\
\hline 2001.73 & 6.449 & 2000.88 & 6.470 & 2000.58 & 6.473 \\
\hline 2001.73 & 6.389 & 2000.88 & 6.430 & 2000.58 & 6.433 \\
\hline 2001.73 & 6.449 & 2000.88 & 6.490 & 2000.58 & 6.473 \\
\hline 2001.73 & 6.429 & 2000.88 & 6.490 & 2000.58 & 6.453 \\
\hline 2001.73 & 6.429 & 2000.88 & 6.490 & 2000.58 & 6.453 \\
\hline 2001.73 & 6.429 & 2000.88 & 6.470 & 2000.58 & 6.433 \\
\hline 2001.73 & 6.429 & 2000.88 & 6.450 & 2000.58 & 6.473 \\
\hline 2001.73 & 6.424 & 2000.88 & 6.490 & 2000.58 & 6.473 \\
\hline 2001.73 & 6.429 & 2000.88 & 6.450 & 2000.58 & 6.493 \\
\hline 2001.73 & 6.409 & 2000.88 & 6.470 & 2000.58 & 6.473 \\
\hline 2001.73 & 6.404 & 2000.88 & 6.510 & 2000.58 & 6.493 \\
\hline 2001.73 & 6.429 & 2000.88 & 6.490 & 2000.58 & 6.493 \\
\hline 2001.73 & 6.404 & 2000.88 & 6.470 & 2000.58 & 6.493 \\
\hline 2001.73 & 6.404 & 2000.88 & 6.490 & 2000.58 & 6.453 \\
\hline 2001.73 & 6.424 & 2000.88 & 6.490 & 2000.58 & 6.473 \\
\hline 2001.73 & 6.469 & 2000.88 & 6.470 & 2000.58 & 6.493 \\
\hline 2001.73 & 6.424 & 2000.88 & 6.490 & 2000.58 & 6.433 \\
\hline 2001.73 & 6.404 & 2000.88 & 6.470 & 2000.58 & 6.473 \\
\hline 4000.01 & 8.447 & 4000.17 & 8.473 & 4000.36 & 8.472 \\
\hline 4000.01 & 8.447 & 4000.17 & 8.453 & 4000.36 & 8.452 \\
\hline 4000.01 & 8.467 & 4000.17 & 8.473 & 4000.36 & 8.452 \\
\hline 4000.01 & 8.447 & 4000.17 & 8.433 & 4000.36 & 8.492 \\
\hline 4000.01 & 8.447 & 4000.17 & 8.493 & 4000.36 & 8.472 \\
\hline 4000.01 & 8.407 & 4000.17 & 8.493 & 4000.36 & 8.472 \\
\hline
\end{tabular}


Table A.1 Data Set 1 - Relative Distance Calibration: $0 \mathrm{~m}$ to $60 \mathrm{~m}$

\begin{tabular}{|l|l|}
\hline Location: Environmentally controlled lab, Bldg. 220 & Location: Environmentally controlled
\end{tabular} No Filter

File: rel_dist_anal.xls

Test dates: July 14-15, 1999

lab, Bldg. 220

No Filter

File: 8_22_99_laser.xls

Test date: Aug. 2, 1999

\begin{tabular}{|c|c|c|c|c|c|}
\hline $\begin{array}{l}\text { Interfero. Dist. } \\
(\mathrm{mm})\end{array}$ & $\begin{array}{l}\text { Yllw, not shiny } \\
\text { (m) }\end{array}$ & $\begin{array}{l}\text { Interfero. Dist. } \\
\qquad(\mathrm{mm})\end{array}$ & $\begin{array}{l}\text { Grn, not shiny } \\
\text { (m) }\end{array}$ & $\begin{array}{l}\text { Interfero. Dist. } \\
\qquad(\mathrm{mm})\end{array}$ & $\begin{array}{c}\text { Grn, not shiny - } \\
\text { recal. } \\
(\mathrm{m})\end{array}$ \\
\hline 4000.01 & 8.447 & 4000.17 & 8.453 & 4000.36 & 8.472 \\
\hline 4000.01 & 8.467 & 4000.17 & 8.433 & 4000.36 & 8.452 \\
\hline 4000.01 & 8.427 & 4000.17 & 8.473 & 4000.36 & 8.472 \\
\hline 4000.01 & 8.467 & 4000.17 & 8.473 & 4000.36 & 8.472 \\
\hline 4000.01 & 8.447 & 4000.17 & 8.473 & 4000.36 & 8.452 \\
\hline 4000.01 & 8.427 & 4000.17 & 8.433 & 4000.36 & 8.472 \\
\hline 4000.01 & 8.447 & 4000.17 & 8.473 & 4000.36 & 8.472 \\
\hline 4000.01 & 8.427 & 4000.17 & 8.493 & 4000.36 & 8.432 \\
\hline 4000.01 & 8.447 & 4000.17 & 8.493 & 4000.36 & 8.492 \\
\hline 4000.01 & 8.447 & 4000.17 & 8.473 & 4000.36 & 8.472 \\
\hline 4000.01 & 8.427 & 4000.17 & 8.453 & 4000.36 & 8.452 \\
\hline 4000.01 & 8.407 & 4000.17 & 8.453 & 4000.36 & 8.472 \\
\hline 4000.01 & 8.427 & 4000.17 & 8.473 & 4000.36 & 8.432 \\
\hline 4000.01 & 8.427 & 4000.17 & 8.473 & 4000.36 & 8.472 \\
\hline 4000.01 & 8.447 & 4000.17 & 8.433 & 4000.36 & 8.512 \\
\hline 4000.01 & 8.447 & 4000.17 & 8.493 & 4000.36 & 8.472 \\
\hline 4000.01 & 8.467 & 4000.17 & 8.433 & 4000.36 & 8.472 \\
\hline 4000.01 & 8.407 & 4000.17 & 8.473 & 4000.36 & 8.492 \\
\hline 4000.01 & 8.407 & 4000.17 & 8.473 & 4000.36 & 8.452 \\
\hline 6000.09 & 10.430 & 6000.18 & 10.456 & 5999.05 & 10.475 \\
\hline 6000.09 & 10.450 & 6000.18 & 10.476 & 5999.05 & 10.490 \\
\hline 6000.09 & 10.470 & 6000.18 & 10.476 & 5999.05 & 10.470 \\
\hline 6000.09 & 10.450 & 6000.18 & 10.476 & 5999.05 & 10.450 \\
\hline 6000.09 & 10.430 & 6000.18 & 10.496 & 5999.05 & 10.470 \\
\hline 6000.09 & 10.410 & 6000.18 & 10.476 & 5999.05 & 10.490 \\
\hline 6000.09 & 10.430 & 6000.18 & 10.476 & 5999.05 & 10.510 \\
\hline 6000.09 & 10.450 & 6000.18 & 10.456 & 5999.05 & 10.490 \\
\hline 6000.09 & 10.430 & 6000.18 & 10.476 & 5999.05 & 10.470 \\
\hline 6000.09 & 10.450 & 6000.18 & 10.496 & 5999.05 & 10.430 \\
\hline 6000.09 & 10.450 & 6000.18 & 10.476 & 5999.05 & 10.490 \\
\hline 6000.09 & 10.450 & 6000.18 & 10.496 & 5999.05 & 10.490 \\
\hline 6000.09 & 10.450 & 6000.18 & 10.496 & 5999.05 & 10.450 \\
\hline 6000.09 & 10.450 & 6000.18 & 10.476 & 5999.05 & 10.450 \\
\hline 6000.09 & 10.470 & 6000.18 & 10.456 & 5999.05 & 10.490 \\
\hline 6000.09 & 10.430 & 6000.18 & 10.516 & 5999.05 & 10.510 \\
\hline 6000.09 & 10.470 & 6000.18 & 10.516 & 5999.05 & 10.450 \\
\hline 6000.09 & 10.390 & 6000.18 & 10.496 & 5999.05 & 10.470 \\
\hline 6000.09 & 10.430 & 6000.18 & 10.496 & 5999.05 & 10.430 \\
\hline 6000.09 & 10.430 & 6000.18 & 10.496 & 5999.05 & 10.490 \\
\hline 6000.09 & 10.410 & 6000.18 & 10.476 & 5999.05 & 10.490 \\
\hline 6000.09 & 10.470 & 6000.18 & 10.456 & 5999.05 & 10.490 \\
\hline 6000.09 & 10.390 & 6000.18 & 10.456 & 5999.05 & 10.450 \\
\hline 6000.09 & 10.430 & 6000.18 & 10.496 & 5999.05 & 10.470 \\
\hline 6000.09 & 10.430 & 6000.18 & 10.496 & 5999.05 & 10.490 \\
\hline 8000.24 & 12.453 & 8001.45 & 12.479 & 8000.17 & 12.493 \\
\hline 8000.24 & 12.453 & 8001.45 & 12.479 & 8000.17 & 12.488 \\
\hline 8000.24 & 12.433 & 8001.45 & 12.479 & 8000.17 & 12.468 \\
\hline 8000.24 & 12.433 & 8001.45 & 12.479 & 8000.17 & 12.488 \\
\hline 8000.24 & 12.433 & 8001.45 & 12.479 & 8000.17 & 12.488 \\
\hline 8000.24 & 12.428 & 8001.45 & 12.479 & 8000.17 & 12.468 \\
\hline 8000.24 & 12.413 & 8001.45 & 12.500 & 8000.17 & 12.488 \\
\hline 8000.24 & 12.433 & 8001.45 & 12.479 & 8000.17 & 12.468 \\
\hline 8000.24 & 12.453 & 8001.45 & 12.479 & 8000.17 & 12.488 \\
\hline 8000.24 & 12.433 & 8001.45 & 12.479 & 8000.17 & 12.468 \\
\hline 8000.24 & 12.433 & 8001.45 & 12.479 & 8000.17 & 12.468 \\
\hline 8000.24 & 12.448 & 8001.45 & 12.479 & 8000.17 & 12.488 \\
\hline 8000.24 & 12.413 & 8001.45 & 12.479 & 8000.17 & 12.488 \\
\hline
\end{tabular}


Table A.1 Data Set 1 - Relative Distance Calibration: $0 \mathrm{~m}$ to $60 \mathrm{~m}$

\begin{tabular}{|l|l|}
\hline Location: Environmentally controlled lab, Bldg. 220 & Location: Environmentally controlled
\end{tabular} No Filter

File: rel_dist_anal.xls

Test dates: July 14-15, 1999

lab, Bldg. 220

No Filter

File: 8_22_99_laser.xls

Test date: Aug. 2, 1999

\begin{tabular}{|c|c|c|c|c|c|}
\hline $\begin{array}{l}\text { Interfero. Dist. } \\
\qquad(\mathrm{mm})\end{array}$ & $\begin{array}{l}\text { Yllw, not shiny } \\
\text { (m) }\end{array}$ & $\begin{array}{l}\text { Interfero. Dist. } \\
\qquad(\mathrm{mm})\end{array}$ & $\begin{array}{l}\text { Grn, not shiny } \\
\text { (m) }\end{array}$ & $\begin{array}{l}\text { Interfero. Dist. } \\
(\mathrm{mm})\end{array}$ & $\begin{array}{c}\text { Grn, not shiny - } \\
\text { recal. } \\
(\mathrm{m})\end{array}$ \\
\hline 8000.24 & 12.453 & 8001.45 & 12.479 & 8000.17 & 12.488 \\
\hline 8000.24 & 12.433 & 8001.45 & 12.479 & 8000.17 & 12.488 \\
\hline 8000.24 & 12.433 & 8001.45 & 12.479 & 8000.17 & 12.488 \\
\hline 8000.24 & 12.433 & 8001.45 & 12.459 & 8000.17 & 12.488 \\
\hline 8000.24 & 12.433 & 8001.45 & 12.479 & 8000.17 & 12.468 \\
\hline 8000.24 & 12.443 & 8001.45 & 12.479 & 8000.17 & 12.468 \\
\hline 8000.24 & 12.413 & 8001.45 & 12.479 & 8000.17 & 12.468 \\
\hline 8000.24 & 12.453 & 8001.45 & 12.479 & 8000.17 & 12.468 \\
\hline 8000.24 & 12.433 & 8001.45 & 12.479 & 8000.17 & 12.468 \\
\hline 8000.24 & 12.428 & 8001.45 & 12.479 & 8000.17 & 12.488 \\
\hline 8000.24 & 12.408 & 8001.45 & 12.479 & 8000.17 & 12.488 \\
\hline 8000.24 & 12.428 & 8001.45 & 12.479 & 8000.17 & 12.448 \\
\hline 9999.92 & 14.432 & 10000.87 & 14.463 & 10002.66 & 14.472 \\
\hline 9999.92 & 14.412 & 10000.87 & 14.463 & 10002.66 & 14.492 \\
\hline 9999.92 & 14.412 & 10000.87 & 14.463 & 10002.66 & 14.492 \\
\hline 9999.92 & 14.412 & 10000.87 & 14.443 & 10002.66 & 14.452 \\
\hline 9999.92 & 14.412 & 10000.87 & 14.483 & 10002.66 & 14.472 \\
\hline 9999.92 & 14.432 & 10000.87 & 14.483 & 10002.66 & 14.432 \\
\hline 9999.92 & 14.412 & 10000.87 & 14.443 & 10002.66 & 14.432 \\
\hline 9999.92 & 14.412 & 10000.87 & 14.463 & 10002.66 & 14.472 \\
\hline 9999.92 & 14.412 & 10000.87 & 14.463 & 10002.66 & 14.472 \\
\hline 9999.92 & 14.412 & 10000.87 & 14.463 & 10002.66 & 14.432 \\
\hline 9999.92 & 14.392 & 10000.87 & 14.463 & 10002.66 & 14.452 \\
\hline 9999.92 & 14.412 & 10000.87 & 14.483 & 10002.66 & 14.492 \\
\hline 9999.92 & 14.412 & 10000.87 & 14.483 & 10002.66 & 14.472 \\
\hline 9999.92 & 14.412 & 10000.87 & 14.483 & 10002.66 & 14.452 \\
\hline 9999.92 & 14.392 & 10000.87 & 14.463 & 10002.66 & 14.472 \\
\hline 9999.92 & 14.412 & 10000.87 & 14.483 & 10002.66 & 14.452 \\
\hline 9999.92 & 14.432 & 10000.87 & 14.483 & 10002.66 & 14.472 \\
\hline 9999.92 & 14.412 & 10000.87 & 14.463 & 10002.66 & 14.452 \\
\hline 9999.92 & 14.432 & 10000.87 & 14.463 & 10002.66 & 14.472 \\
\hline 9999.92 & 14.412 & 10000.87 & 14.483 & 10002.66 & 14.452 \\
\hline 9999.92 & 14.432 & 10000.87 & 14.483 & 10002.66 & 14.492 \\
\hline 9999.92 & 14.412 & 10000.87 & 14.483 & 10002.66 & 14.472 \\
\hline 9999.92 & 14.412 & 10000.87 & 14.423 & 10002.66 & 14.492 \\
\hline 9999.92 & 14.412 & 10000.87 & 14.463 & 10002.66 & 14.432 \\
\hline 9999.92 & 14.412 & 10000.87 & 14.463 & 10002.66 & 14.472 \\
\hline 14996.48 & 19.414 & 14998.16 & 19.445 & 14990.23 & 19.414 \\
\hline 14996.48 & 19.434 & 14998.16 & 19.405 & 14990.23 & 19.390 \\
\hline 14996.48 & 19.434 & 14998.16 & 19.405 & 14990.23 & 19.410 \\
\hline 14996.48 & 19.394 & 14998.16 & 19.405 & 14990.23 & 19.410 \\
\hline 14996.48 & 19.434 & 14998.16 & 19.445 & 14990.23 & 19.410 \\
\hline 14996.48 & 19.454 & 14998.16 & 19.445 & 14990.23 & 19.410 \\
\hline 14996.48 & 19.434 & 14998.16 & 19.405 & 14990.23 & 19.414 \\
\hline 14996.48 & 19.434 & 14998.16 & 19.425 & 14990.23 & 19.394 \\
\hline 14996.48 & 19.414 & 14998.16 & 19.445 & 14990.23 & 19.390 \\
\hline 14996.48 & 19.434 & 14998.16 & 19.445 & 14990.23 & 19.430 \\
\hline 14996.48 & 19.414 & 14998.16 & 19.425 & 14990.23 & 19.410 \\
\hline 14996.48 & 19.414 & 14998.16 & 19.445 & 14990.23 & 19.430 \\
\hline 14996.48 & 19.434 & 14998.16 & 19.425 & 14990.23 & 19.370 \\
\hline 14996.48 & 19.434 & 14998.16 & 19.445 & 14990.23 & 19.430 \\
\hline 14996.48 & 19.434 & 14998.16 & 19.445 & 14990.23 & 19.430 \\
\hline 14996.48 & 19.434 & 14998.16 & 19.405 & 14990.23 & 19.430 \\
\hline 14996.48 & 19.414 & 14998.16 & 19.405 & 14990.23 & 19.430 \\
\hline 14996.48 & 19.414 & 14998.16 & 19.465 & 14990.23 & 19.410 \\
\hline 14996.48 & 19.414 & 14998.16 & 19.405 & 14990.23 & 19.410 \\
\hline 14996.48 & 19.414 & 14998.16 & 19.385 & 14990.23 & 19.410 \\
\hline
\end{tabular}


Table A.1 Data Set 1 - Relative Distance Calibration: $0 \mathrm{~m}$ to $60 \mathrm{~m}$

\begin{tabular}{|l|l|}
\hline Location: Environmentally controlled lab, Bldg. 220 & Location: Environmentally controlled
\end{tabular} No Filter

File: rel_dist_anal.xls

Test dates: July 14-15, 1999

lab, Bldg. 220

No Filter

File: 8_22_99_laser.xls

Test date: Aug. 2, 1999

\begin{tabular}{|c|c|c|c|c|c|}
\hline $\begin{array}{l}\text { Interfero. Dist. } \\
(\mathrm{mm})\end{array}$ & $\begin{array}{l}\text { Yllw, not shiny } \\
\text { (m) }\end{array}$ & $\begin{array}{l}\text { Interfero. Dist. } \\
(\mathrm{mm})\end{array}$ & $\begin{array}{l}\text { Grn, not shiny } \\
\text { (m) }\end{array}$ & $\begin{array}{l}\text { Interfero. Dist. } \\
(\mathrm{mm})\end{array}$ & $\begin{array}{c}\text { Grn, not shiny - } \\
\text { recal. } \\
\text { (m) }\end{array}$ \\
\hline 14996.48 & 19.434 & 14998.16 & 19.385 & 14990.23 & 19.410 \\
\hline 14996.48 & 19.454 & 14998.16 & 19.405 & 14990.23 & 19.430 \\
\hline 14996.48 & 19.434 & 14998.16 & 19.385 & 14990.23 & 19.394 \\
\hline 14996.48 & 19.454 & 14998.16 & 19.385 & 14990.23 & 19.370 \\
\hline 14996.48 & 19.434 & 14998.16 & 19.425 & 14990.23 & 19.390 \\
\hline 20010.77 & 24.434 & 20010.10 & 24.405 & 19990.03 & 24.390 \\
\hline 20010.77 & 24.434 & 20010.10 & 24.445 & 19990.03 & 24.410 \\
\hline 20010.77 & 24.434 & 20010.10 & 24.405 & 19990.03 & 24.390 \\
\hline 20010.77 & 24.434 & 20010.10 & 24.405 & 19990.03 & 24.390 \\
\hline 20010.77 & 24.434 & 20010.10 & 24.405 & 19990.03 & 24.390 \\
\hline 20010.77 & 24.434 & 20010.10 & 24.425 & 19990.03 & 24.390 \\
\hline 20010.77 & 24.454 & 20010.10 & 24.405 & 19990.03 & 24.390 \\
\hline 20010.77 & 24.434 & 20010.10 & 24.425 & 19990.03 & 24.390 \\
\hline 20010.77 & 24.454 & 20010.10 & 24.405 & 19990.03 & 24.390 \\
\hline 20010.77 & 24.474 & 20010.10 & 24.425 & 19990.03 & 24.410 \\
\hline 20010.77 & 24.454 & 20010.10 & 24.425 & 19990.03 & 24.430 \\
\hline 20010.77 & 24.454 & 20010.10 & 24.405 & 19990.03 & 24.370 \\
\hline 20010.77 & 24.454 & 20010.10 & 24.425 & 19990.03 & 24.410 \\
\hline 20010.77 & 24.434 & 20010.10 & 24.445 & 19990.03 & 24.390 \\
\hline 20010.77 & 24.454 & 20010.10 & 24.425 & 19990.03 & 24.390 \\
\hline 20010.77 & 24.545 & 20010.10 & 24.405 & 19990.03 & 24.370 \\
\hline 20010.77 & 24.454 & 20010.10 & 24.445 & 19990.03 & 24.410 \\
\hline 20010.77 & 24.454 & 20010.10 & 24.425 & 19990.03 & 24.370 \\
\hline 20010.77 & 24.434 & 20010.10 & 24.405 & 19990.03 & 24.410 \\
\hline 20010.77 & 24.414 & 20010.10 & 24.425 & 19990.03 & 24.410 \\
\hline 20010.77 & 24.454 & 20010.10 & 24.405 & 19990.03 & 24.410 \\
\hline 20010.77 & 24.454 & 20010.10 & 24.425 & 19990.03 & 24.410 \\
\hline 20010.77 & 24.434 & 20010.10 & 24.425 & 19990.03 & 24.410 \\
\hline 20010.77 & 24.454 & 20010.10 & 24.385 & 19990.03 & 24.390 \\
\hline 20010.77 & 24.454 & 20010.10 & 24.425 & 19990.03 & 24.390 \\
\hline 30006.01 & 34.450 & 30006.19 & 34.425 & 30524.89 & 34.970 \\
\hline 30006.01 & 34.430 & 30006.19 & 34.445 & 30524.89 & 34.950 \\
\hline 30006.01 & 34.445 & 30006.19 & 34.465 & 30524.89 & 34.950 \\
\hline 30006.01 & 34.425 & 30006.19 & 34.405 & 30524.89 & 34.930 \\
\hline 30006.01 & 34.425 & 30006.19 & 34.445 & 30524.89 & 34.950 \\
\hline 30006.01 & 34.445 & 30006.19 & 34.425 & 30524.89 & 34.950 \\
\hline 30006.01 & 34.485 & 30006.19 & 34.485 & 30524.89 & 34.970 \\
\hline 30006.01 & 34.445 & 30006.19 & 34.425 & 30524.89 & 34.970 \\
\hline 30006.01 & 34.465 & 30006.19 & 34.425 & 30524.89 & 34.950 \\
\hline 30006.01 & 34.465 & 30006.19 & 34.425 & 30524.89 & 34.990 \\
\hline 30006.01 & 34.465 & 30006.19 & 34.425 & 30524.89 & 34.970 \\
\hline 30006.01 & 34.445 & 30006.19 & 34.425 & 30524.89 & 34.970 \\
\hline 30006.01 & 34.465 & 30006.19 & 34.465 & 30524.89 & 34.990 \\
\hline 30006.01 & 34.465 & 30006.19 & 34.425 & 30524.89 & 34.970 \\
\hline 30006.01 & 34.445 & 30006.19 & 34.445 & 30524.89 & 34.930 \\
\hline 30006.01 & 34.445 & 30006.19 & 34.445 & 30524.89 & 35.010 \\
\hline 30006.01 & 34.445 & 30006.19 & 34.425 & 30524.89 & 34.930 \\
\hline 30006.01 & 34.445 & 30006.19 & 34.425 & 30524.89 & 34.950 \\
\hline 30006.01 & 34.465 & 30006.19 & 34.425 & 30524.89 & 34.950 \\
\hline 30006.01 & 34.465 & 30006.19 & 34.445 & 30524.89 & 34.930 \\
\hline 30006.01 & 34.465 & 30006.19 & 34.405 & 30524.89 & 34.950 \\
\hline 30006.01 & 34.405 & 30006.19 & 34.445 & 30524.89 & 34.950 \\
\hline 30006.01 & 34.445 & 30006.19 & 34.465 & 30524.89 & 34.990 \\
\hline 30006.01 & 34.465 & 30006.19 & 34.445 & 30524.89 & 35.010 \\
\hline 30006.01 & 34.425 & 30006.19 & 34.385 & 30524.89 & 34.950 \\
\hline 39994.27 & 44.385 & 39994.04 & 44.445 & 0.00 & 4.513 \\
\hline 39994.27 & 44.365 & 39994.04 & 44.405 & 0.00 & 4.513 \\
\hline
\end{tabular}


Table A.1 Data Set 1 - Relative Distance Calibration: $0 \mathrm{~m}$ to $60 \mathrm{~m}$

\begin{tabular}{|l|l|}
\hline Location: Environmentally controlled lab, Bldg. 220 & Location: Environmentally controlled
\end{tabular} No Filter

File: rel_dist_anal.xls

Test dates: July 14-15, 1999

lab, Bldg. 220

No Filter

File: 8_22_99_laser.xls

Test date: Aug. 2, 1999

\begin{tabular}{|c|c|c|c|c|c|}
\hline $\begin{array}{l}\text { Interfero. Dist. } \\
\text { (mm) }\end{array}$ & $\begin{array}{l}\text { Yllw, not shiny } \\
\text { (m) }\end{array}$ & $\begin{array}{l}\text { Interfero. Dist. } \\
\text { (mm) }\end{array}$ & $\begin{array}{l}\text { Grn, not shiny } \\
\text { (m) }\end{array}$ & $\begin{array}{l}\text { Interfero. Dist. } \\
(\mathrm{mm})\end{array}$ & $\begin{array}{c}\text { Grn, not shiny - } \\
\text { recal. } \\
(\mathrm{m})\end{array}$ \\
\hline 39994.27 & 44.405 & 39994.04 & 44.425 & 0.00 & 4.493 \\
\hline 39994.27 & 44.405 & 39994.04 & 44.445 & 0.00 & 4.513 \\
\hline 39994.27 & 44.405 & 39994.04 & 44.465 & 0.00 & 4.473 \\
\hline 39994.27 & 44.365 & 39994.04 & 44.405 & 0.00 & 4.493 \\
\hline 39994.27 & 44.405 & 39994.04 & 44.465 & 0.00 & 4.473 \\
\hline 39994.27 & 44.405 & 39994.04 & 44.465 & 0.00 & 4.493 \\
\hline 39994.27 & 44.425 & 39994.04 & 44.405 & 0.00 & 4.493 \\
\hline 39994.27 & 44.405 & 39994.04 & 44.445 & 0.00 & 4.493 \\
\hline 39994.27 & 44.385 & 39994.04 & 44.465 & 0.00 & 4.513 \\
\hline 39994.27 & 44.365 & 39994.04 & 44.425 & 0.00 & 4.493 \\
\hline 39994.27 & 44.405 & 39994.04 & 44.445 & 0.00 & 4.513 \\
\hline 39994.27 & 44.435 & 39994.04 & 44.405 & 0.00 & 4.513 \\
\hline 39994.27 & 44.405 & 39994.04 & 44.405 & 0.00 & 4.493 \\
\hline 39994.27 & 44.365 & 39994.04 & 44.445 & 0.00 & 4.493 \\
\hline 39994.27 & 44.405 & 39994.04 & 44.445 & 0.00 & 4.493 \\
\hline 39994.27 & 44.405 & 39994.04 & 44.405 & 0.00 & 4.493 \\
\hline 39994.27 & 44.465 & 39994.04 & 44.405 & 0.00 & 4.493 \\
\hline 39994.27 & 44.485 & 39994.04 & 44.385 & 0.00 & 4.493 \\
\hline 39994.27 & 44.405 & 39994.04 & 44.385 & 0.00 & 4.493 \\
\hline 39994.27 & 44.405 & 39994.04 & 44.465 & 0.00 & 4.493 \\
\hline 39994.27 & 44.385 & 39994.04 & 44.425 & 0.00 & 4.513 \\
\hline 39994.27 & 44.385 & 39994.04 & 44.445 & 0.00 & 4.493 \\
\hline 39994.27 & 44.405 & 39994.04 & 44.405 & 0.00 & 4.513 \\
\hline 49952.25 & 54.325 & 49988.89 & 54.445 & 40112.49 & 44.570 \\
\hline 49952.25 & 54.305 & 49988.89 & 54.465 & 40112.49 & 44.585 \\
\hline 49952.25 & 54.305 & 49988.89 & 54.465 & 40112.49 & 44.525 \\
\hline 49952.25 & 54.325 & 49988.89 & 54.485 & 40112.49 & 44.585 \\
\hline 49952.25 & 54.365 & 49988.89 & 54.405 & 40112.49 & 44.585 \\
\hline 49952.25 & 54.325 & 49988.89 & 54.505 & 40112.49 & 44.550 \\
\hline 49952.25 & 54.405 & 49988.89 & 54.385 & 40112.49 & 44.565 \\
\hline 49952.25 & 54.285 & 49988.89 & 54.465 & 40112.49 & 44.605 \\
\hline 49952.25 & 54.305 & 49988.89 & 54.425 & 40112.49 & 44.565 \\
\hline 49952.25 & 54.285 & 49988.89 & 54.425 & 40112.49 & 44.565 \\
\hline 49952.25 & 54.305 & 49988.89 & 54.485 & 40112.49 & 44.585 \\
\hline 49952.25 & 54.365 & 49988.89 & 54.485 & 40112.49 & 44.585 \\
\hline 49952.25 & 54.305 & 49988.89 & 54.465 & 40112.49 & 44.585 \\
\hline 49952.25 & 54.325 & 49988.89 & 54.465 & 40112.49 & 44.605 \\
\hline 49952.25 & 54.285 & 49988.89 & 54.465 & 40112.49 & 44.570 \\
\hline 49952.25 & 54.325 & 49988.89 & 54.405 & 40112.49 & 44.605 \\
\hline 49952.25 & 54.385 & 49988.89 & 54.505 & 40112.49 & 44.590 \\
\hline 49952.25 & 54.305 & 49988.89 & 54.445 & 40112.49 & 44.625 \\
\hline 49952.25 & 54.325 & 49988.89 & 54.465 & 40112.49 & 44.565 \\
\hline 49952.25 & 54.305 & 49988.89 & 54.505 & 40112.49 & 44.610 \\
\hline 49952.25 & 54.305 & 49988.89 & 54.445 & 40112.49 & 44.565 \\
\hline 49952.25 & 54.325 & 49988.89 & 54.465 & 40112.49 & 44.630 \\
\hline 49952.25 & 54.325 & 49988.89 & 54.445 & 40112.49 & 44.590 \\
\hline 49952.25 & 54.345 & 49988.89 & 54.425 & 40112.49 & 44.585 \\
\hline 49952.25 & 54.305 & 49988.89 & 54.425 & 40112.49 & 44.625 \\
\hline & & & & 49066.14 & 53.585 \\
\hline & & & & 49066.14 & 53.585 \\
\hline & & & & 49066.14 & 53.565 \\
\hline & & & & 49066.14 & 53.545 \\
\hline & & & & 49066.14 & 53.505 \\
\hline & & & & 49066.14 & 53.585 \\
\hline & & & & 49066.14 & 53.545 \\
\hline & & & & 49066.14 & 53.565 \\
\hline & & & & 49066.14 & 53.605 \\
\hline
\end{tabular}


Table A.1 Data Set 1 - Relative Distance Calibration: $0 \mathrm{~m}$ to $60 \mathrm{~m}$

\begin{tabular}{|l|l|}
\hline Location: Environmentally controlled lab, Bldg. 220 & Location: Environmentally controlled
\end{tabular} No Filter

File: rel_dist_anal.xls

Test dates: July 14-15, 1999

lab, Bldg. 220

No Filter

File: 8_22_99_laser.xls

Test date: Aug. 2, 1999

\begin{tabular}{|c|c|c|c|c|c|}
\hline $\begin{array}{l}\text { Interfero. Dist. } \\
\quad(\mathrm{mm})\end{array}$ & $\begin{array}{l}\text { Yllw, not shiny } \\
\text { (m) }\end{array}$ & $\begin{array}{l}\text { Interfero. Dist. } \\
\quad(\mathrm{mm})\end{array}$ & $\begin{array}{l}\text { Grn, not shiny } \\
\text { (m) }\end{array}$ & $\begin{array}{l}\text { Interfero. Dist. } \\
\quad(\mathrm{mm})\end{array}$ & $\begin{array}{l}\text { Grn, not shiny - } \\
\text { recal. } \\
\text { (m) }\end{array}$ \\
\hline & & & & $\begin{array}{l}49066.14 \\
49066.14 \\
49066.14 \\
49066.14 \\
49066.14 \\
49066.14 \\
49066.14 \\
49066.14 \\
49066.14 \\
49066.14 \\
49066.14 \\
49066.14 \\
49066.14 \\
49066.14 \\
49066.14 \\
49066.14\end{array}$ & $\begin{array}{l}53.605 \\
53.545 \\
53.645 \\
53.565 \\
53.605 \\
53.625 \\
53.625 \\
53.565 \\
53.565 \\
53.545 \\
53.565 \\
53.565 \\
53.545 \\
53.565 \\
53.565 \\
53.525\end{array}$ \\
\hline
\end{tabular}




\section{APPENDIX B - Absolute Distance Measurements}

Table B.1 Data Set 2 - Absolute Distance Calibration, $30 \mathrm{~m}$ and $60 \mathrm{~m}$

\begin{tabular}{|c|c|c|c|c|}
\hline $\begin{array}{l}\text { Location: Env } \\
\text { No Filter } \\
\text { Test date: July }\end{array}$ & ly controlle & dg. 220 & & abs_dist.xls \\
\hline $\begin{array}{c}\text { Distance } \\
\text { (m) }\end{array}$ & $\begin{array}{c}\text { Laser (m) } \\
\text { Run } 1\end{array}$ & $\begin{array}{c}\text { Laser (m) } \\
\text { Run } 2\end{array}$ & $\begin{array}{l}\text { Distance } \\
\text { (m) }\end{array}$ & $\begin{array}{l}\text { Laser } \\
(\mathrm{m})\end{array}$ \\
\hline 30.48 & 30.430 & 30.474 & 60.96 & 61.005 \\
\hline 30.48 & 30.430 & 30.474 & 60.96 & 60.985 \\
\hline 30.48 & 30.450 & 30.454 & 60.96 & 61.045 \\
\hline 30.48 & 30.470 & 30.490 & 60.96 & 61.045 \\
\hline 30.48 & 30.450 & 30.474 & 60.96 & 61.025 \\
\hline 30.48 & 30.470 & 30.474 & 60.96 & 60.985 \\
\hline 30.48 & 30.430 & 30.450 & 60.96 & 61.065 \\
\hline 30.48 & 30.430 & 30.470 & 60.96 & 61.045 \\
\hline 30.48 & 30.470 & 30.470 & 60.96 & 61.045 \\
\hline 30.48 & 30.490 & 30.454 & 60.96 & 61.025 \\
\hline 30.48 & 30.470 & 30.470 & 60.96 & 61.045 \\
\hline 30.48 & 30.450 & 30.470 & 60.96 & 61.025 \\
\hline 30.48 & 30.470 & 30.494 & 60.96 & 61.025 \\
\hline 30.48 & 30.470 & 30.450 & 60.96 & 60.985 \\
\hline 30.48 & 30.470 & 30.470 & 60.96 & 61.045 \\
\hline 30.48 & 30.470 & 30.490 & 60.96 & 61.025 \\
\hline 30.48 & 30.490 & 30.434 & 60.96 & 61.065 \\
\hline 30.48 & 30.450 & 30.450 & 60.96 & 61.045 \\
\hline 30.48 & 30.470 & 30.470 & 60.96 & 61.045 \\
\hline 30.48 & 30.470 & 30.450 & 60.96 & 61.005 \\
\hline 30.48 & 30.470 & 30.470 & 60.96 & 61.045 \\
\hline 30.48 & 30.470 & 30.454 & 60.96 & 60.985 \\
\hline 30.48 & 30.470 & 30.454 & 60.96 & 61.005 \\
\hline 30.48 & 30.470 & 30.470 & 60.96 & 61.045 \\
\hline 30.48 & 30.410 & 30.470 & 60.96 & 61.025 \\
\hline
\end{tabular}

Table B.2 Data Set 3 - Absolute Distance Calibration, $60 \mathrm{~m}$ to $108 \mathrm{~m}$

\begin{tabular}{|c|c|c|c|}
\hline $\begin{array}{l}\text { Location: Attic, Bld } \\
\text { No Filter } \\
\text { File: } 100 \text { mcalib.xls } \\
\text { Test Date: July } 28,1\end{array}$ & & $\begin{array}{l}\text { Location: Attic, Bldg } \\
\text { No Filter } \\
\text { File: } 108 \text { m_calib.xls } \\
\text { Test Date: August 2, }\end{array}$ & \\
\hline $\begin{array}{c}\text { Tape Distance } \\
(\mathrm{m})\end{array}$ & $\begin{array}{l}\text { Wht, not shiny } \\
(\mathrm{m})\end{array}$ & $\begin{array}{c}\text { Tape Distance } \\
(\mathrm{m})\end{array}$ & $\begin{array}{l}\text { Wht., not shiny } \\
\text { (m) }\end{array}$ \\
\hline 60 & 60.066 & 108.00 & 108.019 \\
\hline 60 & 59.986 & 108.00 & 108.019 \\
\hline 60 & 60.066 & 108.00 & 108.054 \\
\hline 60 & 60.046 & 108.00 & 108.054 \\
\hline 60 & 60.026 & 108.00 & 108.039 \\
\hline 60 & 60.026 & 108.00 & 108.014 \\
\hline 60 & 60.026 & 108.00 & 108.014 \\
\hline 60 & 60.046 & 108.00 & 108.034 \\
\hline 60 & 60.046 & 108.00 & 108.074 \\
\hline 60 & 60.006 & 108.00 & 108.054 \\
\hline 60 & 60.046 & 108.00 & 108.034 \\
\hline 60 & 60.066 & 108.00 & 108.014 \\
\hline 60 & 60.026 & 108.00 & 108.014 \\
\hline 60 & 60.066 & 108.00 & 108.034 \\
\hline 60 & 60.046 & 108.00 & 108.034 \\
\hline 60 & 60.026 & 108.00 & 108.034 \\
\hline
\end{tabular}


Table B.2 Data Set 3 - Absolute Distance Calibration, $60 \mathrm{~m}$ to $108 \mathrm{~m}$

\begin{tabular}{l|l}
\hline Location: Attic, Bldg. 226 & Location: Attic, Bldg. 226
\end{tabular}

No Filter

File: 100 mcalib.xls

Test Date: July 28, 1999

No Filter

File: 108m_calib.xls

\begin{tabular}{|c|c|c|c|}
\hline Date. Jury 28,1 & & Test Date: August 2, & \\
\hline $\begin{array}{l}\text { Tape Distance } \\
(\mathrm{m})\end{array}$ & $\begin{array}{l}\text { Wht, not shiny } \\
(\mathrm{m})\end{array}$ & $\begin{array}{c}\text { Tape Distance } \\
(\mathrm{m})\end{array}$ & $\begin{array}{l}\text { Wht., not shiny } \\
\text { (m) }\end{array}$ \\
\hline 60 & 60.046 & 108.00 & 108.054 \\
\hline 60 & 60.046 & 108.00 & 108.074 \\
\hline 60 & 60.026 & 108.00 & 108.034 \\
\hline 60 & 60.022 & 108.00 & 108.014 \\
\hline 60 & 60.022 & 108.00 & 108.074 \\
\hline 60 & 60.022 & 108.00 & 108.014 \\
\hline 60 & 60.042 & 108.00 & 108.054 \\
\hline 60 & 60.022 & 108.00 & 108.054 \\
\hline 60 & 60.046 & 108.00 & 108.034 \\
\hline 80 & 79.982 & 100.00 & 99.954 \\
\hline 80 & 80.002 & 100.00 & 100.010 \\
\hline 80 & 80.002 & 100.00 & 99.970 \\
\hline 80 & 80.002 & 100.00 & 100.030 \\
\hline 80 & 80.022 & 100.00 & 100.010 \\
\hline 80 & 80.062 & 100.00 & 100.010 \\
\hline 80 & 80.002 & 100.00 & 99.990 \\
\hline 80 & 80.062 & 100.00 & 99.970 \\
\hline 80 & 80.022 & 100.00 & 99.950 \\
\hline 80 & 80.042 & 100.00 & 99.970 \\
\hline 80 & 80.042 & 100.00 & 100.005 \\
\hline 80 & 80.042 & 100.00 & 99.985 \\
\hline 80 & 80.042 & 100.00 & 99.965 \\
\hline 80 & 80.082 & 100.00 & 100.005 \\
\hline 80 & 80.042 & 100.00 & 100.005 \\
\hline 80 & 80.062 & 100.00 & 99.985 \\
\hline 80 & 80.062 & 100.00 & 100.025 \\
\hline 80 & 80.022 & 100.00 & 100.005 \\
\hline 80 & 79.982 & 100.00 & 100.025 \\
\hline 80 & 80.042 & 100.00 & 100.025 \\
\hline 80 & 80.042 & 100.00 & 100.005 \\
\hline 80 & 80.042 & 100.00 & 99.985 \\
\hline 80 & 80.022 & 100.00 & 99.945 \\
\hline 80 & 80.082 & 100.00 & 100.005 \\
\hline 80 & 80.062 & 100.00 & 99.985 \\
\hline 100 & 100.062 & & \\
\hline 100 & 100.122 & & \\
\hline 100 & 100.182 & & \\
\hline 100 & 100.162 & & \\
\hline 100 & 100.162 & & \\
\hline 100 & 100.182 & & \\
\hline 100 & 100.102 & & \\
\hline 100 & 100.142 & & \\
\hline 100 & 100.062 & & \\
\hline 100 & 100.162 & & \\
\hline 100 & 100.162 & & \\
\hline 100 & 100.102 & & \\
\hline 100 & 100.102 & & \\
\hline 100 & 100.162 & & \\
\hline 100 & 100.162 & & \\
\hline 100 & 100.142 & & \\
\hline 100 & 100.142 & & \\
\hline 100 & 100.122 & & \\
\hline 100 & 100.102 & & \\
\hline
\end{tabular}


Table B.2 Data Set 3 - Absolute Distance Calibration, $60 \mathrm{~m}$ to $108 \mathrm{~m}$ \begin{tabular}{l|l}
\hline Location: Attic, Bldg. 226 & Location: Attic, Bldg. 226
\end{tabular}

No Filter

File: 100mcalib.xls

Test Date: July 28, 1999

No Filter

\begin{tabular}{|c|c|c|c|}
\hline Test Date: July 28,1 & & Test Date: August 2, & \\
\hline $\begin{array}{c}\text { Tape Distance } \\
(\mathrm{m})\end{array}$ & $\begin{array}{l}\text { Wht, not shiny } \\
(\mathrm{m})\end{array}$ & $\begin{array}{c}\text { Tape Distance } \\
(\mathrm{m})\end{array}$ & $\begin{array}{l}\text { Wht., not shiny } \\
\text { (m) }\end{array}$ \\
\hline 100 & 100.082 & & \\
\hline 100 & 100.182 & & \\
\hline 100 & 100.062 & & \\
\hline 100 & 100.122 & & \\
\hline 100 & 100.102 & & \\
\hline 100 & 100.142 & & \\
\hline $108^{\dagger}$ & 109.182 & & \\
\hline 108 & 109.182 & & \\
\hline 108 & 109.177 & & \\
\hline 108 & 109.157 & & \\
\hline 108 & 109.157 & & \\
\hline 108 & 109.197 & & \\
\hline 108 & 109.137 & & \\
\hline 108 & 109.217 & & \\
\hline 108 & 109.157 & & \\
\hline 108 & 109.197 & & \\
\hline 108 & 109.157 & & \\
\hline 108 & 109.177 & & \\
\hline 108 & 109.157 & & \\
\hline 108 & 109.197 & & \\
\hline 108 & 109.197 & & \\
\hline 108 & 109.177 & & \\
\hline 108 & 109.177 & & \\
\hline 108 & 109.177 & & \\
\hline 108 & 109.137 & & \\
\hline 108 & 109.117 & & \\
\hline 108 & 109.197 & & \\
\hline 108 & 109.197 & & \\
\hline 108 & 109.137 & & \\
\hline 108 & 109.117 & & \\
\hline 108 & 109.137 & & \\
\hline
\end{tabular}


Table B.3 Data Set 4 - Absolute Distance Calibration: $5 \mathrm{~m}$ to $161 \mathrm{~m}$

\begin{tabular}{|c|c|c|c|c|c|c|}
\hline \multicolumn{7}{|c|}{$\begin{array}{l}\text { Location: } \text { Hallway from Bldg. } 226 \text { to Comstar } \\
\text { Test date: August 6, } 1999 \text { - white target } \\
\text { August 10,1999 - green and black targets }\end{array}$} \\
\hline \multirow[b]{2}{*}{$\begin{array}{l}\text { Distance } \\
\text { (m) }\end{array}$} & \multicolumn{2}{|c|}{ Without Filter } & \multirow[b]{2}{*}{$\begin{array}{l}\text { Distance } \\
\text { (m) }\end{array}$} & \multicolumn{3}{|c|}{ With Filter } \\
\hline & $\begin{array}{l}\text { Blk not shny } \\
\text { (m) }\end{array}$ & $\begin{array}{l}\text { Grn not shny } \\
\text { (m) }\end{array}$ & & $\begin{array}{l}\text { Blk, not shny } \\
\text { (m) }\end{array}$ & $\begin{array}{l}\text { Grn. not shny } \\
\text { (m) }\end{array}$ & $\begin{array}{l}\text { Wht not shny } \\
\text { (m) }\end{array}$ \\
\hline 161.079 & 161.039 & 161.104 & 161.0790 & NA & NA & 161.134 \\
\hline 161.079 & 161.159 & 161.204 & 161.0790 & NA & NA & 161.474 \\
\hline 161.079 & 161.159 & 161.104 & 161.0790 & NA & NA & 161.094 \\
\hline 161.079 & 160.979 & 161.004 & 161.0790 & NA & NA & 161.074 \\
\hline 161.079 & 161.019 & 161.304 & 161.0790 & NA & NA & 161.034 \\
\hline 161.079 & 161.019 & 161.184 & 161.0790 & NA & $\mathrm{NA}$ & 162.034 \\
\hline 161.079 & 161.199 & 161.204 & 161.0790 & NA & NA & 161.854 \\
\hline 161.079 & 161.139 & 161.124 & 161.0790 & NA & NA & 161.594 \\
\hline 161.079 & 160.999 & 161.244 & 161.0790 & NA & NA & 161.794 \\
\hline 161.079 & 161.099 & 161.244 & 161.0790 & NA & NA & 161.150 \\
\hline 141.059 & 141.145 & 140.990 & 151.071 & 151.219 & 151.179 & 151.130 \\
\hline 141.059 & 141.085 & 140.945 & 151.071 & 151.159 & 151.239 & 151.110 \\
\hline 141.059 & 141.045 & 140.985 & 151.071 & 151.419 & 151.059 & 151.150 \\
\hline 141.059 & 141.085 & 140.965 & 151.071 & 151.039 & 151.239 & 151.110 \\
\hline 141.059 & 141.105 & 140.945 & 151.071 & 151.419 & 151.159 & 151.030 \\
\hline 141.059 & 141.125 & 140.985 & 151.071 & 151.299 & 151.199 & 151.070 \\
\hline 141.059 & 141.110 & 141.045 & 151.071 & 151.219 & 151.099 & 151.150 \\
\hline 141.059 & 141.210 & 141.085 & 151.071 & 151.559 & 151.179 & 151.110 \\
\hline 141.059 & 141.105 & 141.045 & 151.071 & 151.259 & 151.259 & 151.070 \\
\hline 141.059 & 141.170 & 141.025 & 151.071 & 151.219 & 151.074 & 151.150 \\
\hline 120.000 & 120.065 & 119.965 & 141.059 & 141.294 & 141.054 & 141.070 \\
\hline 120.000 & 120.105 & 119.965 & 141.059 & 141.094 & 141.149 & 141.085 \\
\hline 120.000 & 120.105 & 119.985 & 141.059 & 141.174 & 140.994 & 141.165 \\
\hline 120.000 & 120.065 & 119.965 & 141.059 & 141.294 & 141.114 & 141.085 \\
\hline 120.000 & 120.045 & 119.945 & 141.059 & 141.394 & 141.114 & 141.065 \\
\hline 120.000 & 120.105 & 119.965 & 141.059 & 141.294 & 141.174 & 141.085 \\
\hline 120.000 & 120.065 & 119.965 & 141.059 & 141.194 & 141.074 & 141.085 \\
\hline 120.000 & 120.125 & 119.965 & 141.059 & 141.294 & 141.154 & 141.045 \\
\hline 120.000 & 120.065 & 120.165 & 141.059 & 141.234 & 141.034 & 141.105 \\
\hline 120.000 & 120.025 & 119.985 & 141.059 & 141.334 & 141.434 & 141.165 \\
\hline 79.990 & 80.010 & 80.070 & 129.989 & 130.074 & 129.234 & 130.045 \\
\hline 79.990 & 79.970 & 80.025 & 129.989 & 129.994 & 129.934 & 130.045 \\
\hline 79.990 & 80.025 & 80.010 & 129.989 & 129.974 & 130.014 & 130.025 \\
\hline 79.990 & 80.010 & 80.025 & 129.989 & 130.114 & 130.014 & 129.985 \\
\hline 79.990 & 80.030 & 80.050 & 129.989 & 129.974 & 129.974 & 130.005 \\
\hline 79.990 & 80.010 & 80.025 & 129.989 & 130.054 & 130.014 & 130.045 \\
\hline 79.990 & 80.030 & 80.025 & 129.989 & 129.934 & 130.054 & 130.085 \\
\hline 79.990 & 79.990 & 80.070 & 129.989 & 129.994 & 130.074 & 129.965 \\
\hline 79.990 & 80.005 & 80.045 & 129.989 & 129.994 & 129.954 & 130.045 \\
\hline 79.990 & 79.990 & 80.010 & 129.989 & 129.874 & 130.094 & 130.045 \\
\hline 60.596 & 60.565 & 60.630 & 120.000 & 119.994 & 120.054 & 120.000 \\
\hline 60.596 & 60.585 & 60.625 & 120.000 & 120.030 & 120.050 & 120.020 \\
\hline 60.596 & 60.545 & 60.605 & 120.000 & 120.094 & 120.054 & 120.000 \\
\hline 60.596 & 60.565 & 60.625 & 120.000 & 119.950 & 120.054 & 120.040 \\
\hline 60.596 & 60.585 & 60.665 & 120.000 & 120.010 & 120.030 & 120.020 \\
\hline 60.596 & 60.525 & 60.605 & 120.000 & 120.014 & 120.114 & 120.000 \\
\hline 60.596 & 60.505 & 60.625 & 120.000 & 119.990 & 120.054 & 119.980 \\
\hline 60.596 & 60.545 & 60.605 & 120.000 & 120.070 & 120.014 & 120.000 \\
\hline 60.596 & 60.585 & 60.625 & 120.000 & 120.110 & 120.014 & 120.040 \\
\hline 60.596 & 60.565 & 60.625 & 120.000 & 119.950 & 120.034 & 120.040 \\
\hline 50.425 & 50.405 & 50.445 & 120.000 & NA & NA & 120.080 \\
\hline 50.425 & 50.425 & 50.445 & 120.000 & NA & NA & 120.040 \\
\hline 50.425 & 50.425 & 50.425 & 120.000 & NA & NA & 120.020 \\
\hline 50.425 & 50.425 & 50.445 & 120.000 & NA & NA & 120.040 \\
\hline 50.425 & 50.425 & 50.465 & 120.000 & NA & NA & 120.020 \\
\hline 50.425 & 50.385 & 50.425 & 120.000 & NA & NA & 120.040 \\
\hline 50.425 & 50.425 & 50.425 & 120.000 & NA & NA & 120.040 \\
\hline 50.425 & 50.405 & 50.425 & 120.000 & NA & NA & 120.020 \\
\hline 50.425 & 50.425 & 50.445 & 120.000 & NA & NA & 119.980 \\
\hline
\end{tabular}


Table B.3 Data Set 4 - Absolute Distance Calibration: $5 \mathrm{~m}$ to $161 \mathrm{~m}$

\begin{tabular}{|c|c|c|c|c|c|c|}
\hline $\begin{array}{l}\text { Location: } \mathrm{H} \\
\text { Test date: }\end{array}$ & $\begin{array}{l}\text { ay from Bldg. } \\
\text { t } 6,1999-\text { wl } \\
10,1999-g\end{array}$ & $\begin{array}{l}6 \text { to Comstar } \\
\text { target } \\
\text { and black to }\end{array}$ & $\mathrm{Bl}$ & $\begin{array}{r}\text { nd Green Targ } \\
\text { White T }\end{array}$ & $\begin{array}{l}\text {-File: } 5 \text { mto } 16 \\
\text { et - File: } 5 \text { to } 1\end{array}$ & $\begin{array}{l}\mathrm{n} \_8 \_10 \_99 . x \mathrm{ls} \\
\mathrm{m} \_8 \_6 \_99 . x \mathrm{sl}\end{array}$ \\
\hline & With & Filter & & & With Filter & \\
\hline $\begin{array}{l}\text { Distance } \\
(\mathrm{m})\end{array}$ & $\begin{array}{l}\text { Blk not shny } \\
\text { (m) }\end{array}$ & $\begin{array}{c}\text { Grn not shny } \\
\text { (m) }\end{array}$ & $\begin{array}{l}\text { Distance } \\
(\mathrm{m})\end{array}$ & $\begin{array}{l}\text { Blk, not shny } \\
\text { (m) }\end{array}$ & $\begin{array}{l}\text { Grn. not shny } \\
\text { (m) }\end{array}$ & $\begin{array}{l}\text { Wht not shny } \\
\text { (m) }\end{array}$ \\
\hline 50.425 & 50.425 & 50.425 & 120.000 & NA & NA & 120.020 \\
\hline 39.998 & 40.005 & 39.985 & 109.990 & 110.090 & 109.950 & 110.020 \\
\hline 39.998 & 40.045 & 40.025 & 109.990 & 109.990 & 110.050 & 110.060 \\
\hline 39.998 & 40.020 & 40.025 & 109.990 & 110.070 & 110.050 & 110.060 \\
\hline 39.998 & 40.060 & 40.025 & 109.990 & 110.010 & 109.990 & 110.040 \\
\hline 39.998 & 40.020 & 40.025 & 109.990 & 109.970 & 110.050 & 110.020 \\
\hline 39.998 & 40.000 & 40.025 & 109.990 & 109.990 & 110.070 & 110.020 \\
\hline 39.998 & 40.060 & 40.025 & 109.990 & 110.090 & 109.970 & 110.040 \\
\hline 39.998 & 40.020 & 40.025 & 109.990 & 110.050 & 110.030 & 110.080 \\
\hline 39.998 & 40.000 & 40.025 & 109.990 & 110.050 & 110.030 & 110.040 \\
\hline 39.998 & 40.000 & 40.045 & 109.990 & 109.990 & 110.030 & 110.080 \\
\hline 30.097 & 30.140 & 30.120 & 99.993 & 99.990 & 100.010 & 100.056 \\
\hline 30.097 & 30.140 & 30.100 & 99.993 & 100.010 & 100.030 & 100.056 \\
\hline 30.097 & 30.120 & 30.120 & 99.993 & 100.010 & 99.970 & 100.056 \\
\hline 30.097 & 30.140 & 30.120 & 99.993 & 100.030 & 100.010 & 100.076 \\
\hline 30.097 & 30.180 & 30.120 & 99.993 & 100.050 & 100.030 & 100.056 \\
\hline 30.097 & 30.140 & 30.120 & 99.993 & 100.050 & 100.010 & 100.096 \\
\hline 30.097 & 30.140 & 30.140 & 99.993 & 100.030 & 99.970 & 100.016 \\
\hline 30.097 & 30.120 & 30.140 & 99.993 & 100.070 & 100.010 & 100.076 \\
\hline 30.097 & 30.160 & 30.120 & 99.993 & 100.010 & 100.030 & 100.076 \\
\hline 30.097 & 30.140 & 30.120 & 99.993 & 100.010 & 100.010 & 100.076 \\
\hline 19.975 & 20.020 & 19.985 & 79.990 & 80.025 & 80.005 & 80.011 \\
\hline 19.975 & 20.040 & 19.985 & 79.990 & 79.945 & 80.005 & 80.031 \\
\hline 19.975 & 20.020 & 20.005 & 79.990 & 80.050 & 80.005 & 79.991 \\
\hline 19.975 & 20.005 & 19.965 & 79.990 & 80.010 & 80.050 & 80.011 \\
\hline 19.975 & 20.045 & 20.005 & 79.990 & 80.005 & 79.970 & 80.031 \\
\hline 19.975 & 19.985 & 19.985 & 79.990 & 80.030 & 79.985 & 80.011 \\
\hline 19.975 & 20.045 & 20.005 & 79.990 & 80.090 & 80.010 & 80.011 \\
\hline 19.975 & 20.045 & 20.005 & 79.990 & 79.970 & 80.010 & 79.991 \\
\hline 19.975 & 20.020 & 19.985 & 79.990 & 79.970 & 79.985 & 80.011 \\
\hline 19.975 & 20.045 & 19.985 & 79.990 & 80.050 & 79.965 & 80.051 \\
\hline 10.002 & 10.047 & 10.031 & 60.596 & 60.605 & 60.585 & 60.631 \\
\hline 10.002 & 10.067 & 10.011 & 60.596 & 60.605 & 60.585 & 60.611 \\
\hline 10.002 & 10.027 & 9.991 & 60.596 & 60.665 & 60.605 & 60.611 \\
\hline 10.002 & 10.067 & 10.031 & 60.596 & 60.645 & 60.550 & 60.551 \\
\hline 10.002 & 10.067 & 10.011 & 60.596 & 60.665 & 60.605 & 60.591 \\
\hline 10.002 & 10.047 & 10.031 & 60.596 & 60.665 & 60.605 & 60.611 \\
\hline 10.002 & 10.007 & 10.031 & 60.596 & 60.625 & 60.585 & 60.611 \\
\hline 10.002 & 10.047 & 10.011 & 60.596 & 60.645 & 60.545 & 60.651 \\
\hline 10.002 & 10.047 & 10.031 & 60.596 & 60.625 & 60.565 & 60.611 \\
\hline 10.002 & 10.067 & 10.031 & 60.596 & 60.645 & 60.585 & 60.611 \\
\hline 5.009 & 5.040 & 5.000 & 50.425 & 50.485 & 50.445 & 50.471 \\
\hline 5.009 & 5.040 & 5.000 & 50.425 & 50.485 & 50.465 & 50.471 \\
\hline 5.009 & 5.040 & 5.020 & 50.425 & 50.465 & 50.425 & 50.451 \\
\hline 5.009 & 5.060 & 5.004 & 50.425 & 50.425 & 50.465 & 50.471 \\
\hline 5.009 & 5.040 & 5.040 & 50.425 & 50.465 & 50.425 & 50.431 \\
\hline 5.009 & 5.020 & 5.000 & 50.425 & 50.385 & 50.465 & 50.451 \\
\hline 5.009 & 5.020 & 5.000 & 50.425 & 50.445 & 50.445 & 50.471 \\
\hline 5.009 & 5.040 & 5.020 & 50.425 & 50.425 & 50.465 & 50.451 \\
\hline 5.009 & 5.040 & 4.979 & 50.425 & 50.485 & 50.465 & 50.471 \\
\hline 5.009 & 5.040 & 5.020 & 50.425 & 50.465 & 50.465 & 50.491 \\
\hline & & & 39.998 & 40.025 & 40.045 & 40.031 \\
\hline & & & 39.998 & 40.025 & 40.045 & 40.031 \\
\hline & & & 39.998 & 40.045 & 40.045 & 40.051 \\
\hline & & & 39.998 & 40.005 & 40.025 & 40.031 \\
\hline & & & 39.998 & 40.025 & 40.025 & 40.031 \\
\hline & & & 39.998 & 40.025 & 40.045 & 40.011 \\
\hline & & & 39.998 & 40.045 & 40.045 & 40.031 \\
\hline & & & 39.998 & 40.060 & 40.045 & 40.051 \\
\hline & & & 39.998 & 40.025 & 40.040 & 40.011 \\
\hline & & & 39.998 & 40.045 & 40.045 & 40.051 \\
\hline
\end{tabular}


Table B.3 Data Set 4 - Absolute Distance Calibration: $5 \mathrm{~m}$ to $161 \mathrm{~m}$

\begin{tabular}{|c|c|c|c|c|c|c|}
\hline \multicolumn{7}{|c|}{$\begin{array}{l}\text { Location: } \text { Hallway from Bldg. } 226 \text { to Comstar } \\
\text { Test date: August 6, 1999 - white target } \\
\text { August 10,1999 - green and black targets }\end{array}$} \\
\hline \multirow[b]{2}{*}{$\begin{array}{c}\text { Distance } \\
(\mathrm{m})\end{array}$} & \multicolumn{2}{|c|}{ Without Filter } & \multirow[b]{2}{*}{$\begin{array}{l}\text { Distance } \\
(\mathrm{m})\end{array}$} & \multicolumn{3}{|c|}{ With Filter } \\
\hline & $\begin{array}{l}\text { Blk not shny } \\
\text { (m) }\end{array}$ & $\begin{array}{c}\text { Grn not shny } \\
\text { (m) }\end{array}$ & & $\begin{array}{l}\text { Blk, not shny } \\
\text { (m) }\end{array}$ & $\begin{array}{l}\text { Grn. not shny } \\
\text { (m) }\end{array}$ & $\begin{array}{l}\text { Wht not shny } \\
\text { (m) }\end{array}$ \\
\hline & & & $\begin{array}{l}30.097 \\
30.097 \\
30.097 \\
30.097 \\
30.097 \\
30.097 \\
30.097 \\
30.097 \\
30.097 \\
30.097 \\
19.975 \\
19.975 \\
19.975 \\
19.975 \\
19.975 \\
19.975 \\
19.975 \\
19.975 \\
19.975 \\
19.975 \\
10.002 \\
10.002 \\
10.002 \\
10.002 \\
10.002 \\
10.002 \\
10.002 \\
10.002 \\
10.002 \\
10.002 \\
5.009 \\
5.009 \\
5.009 \\
5.009 \\
5.009 \\
5.009 \\
5.009 \\
5.009 \\
5.009 \\
5.009\end{array}$ & $\begin{array}{c}30.100 \\
30.080 \\
30.120 \\
30.080 \\
30.060 \\
30.160 \\
30.140 \\
30.120 \\
30.120 \\
30.100 \\
19.985 \\
19.980 \\
19.980 \\
19.965 \\
19.985 \\
19.965 \\
19.965 \\
19.945 \\
19.985 \\
19.985 \\
9.966 \\
9.966 \\
9.966 \\
9.966 \\
10.007 \\
9.986 \\
9.986 \\
10.027 \\
10.007 \\
10.007 \\
4.959 \\
4.979 \\
4.959 \\
5.000 \\
4.979 \\
5.000 \\
5.000 \\
4.979 \\
4.959 \\
5.000 \\
\end{array}$ & $\begin{array}{l}30.140 \\
30.120 \\
30.160 \\
30.140 \\
30.120 \\
30.120 \\
30.140 \\
30.160 \\
30.160 \\
30.140 \\
20.025 \\
20.025 \\
20.025 \\
20.025 \\
20.025 \\
20.005 \\
20.025 \\
20.025 \\
19.985 \\
20.045 \\
10.051 \\
10.051 \\
10.051 \\
10.051 \\
10.051 \\
10.051 \\
10.051 \\
10.011 \\
10.011 \\
10.011 \\
5.040 \\
5.040 \\
5.020 \\
5.040 \\
5.040 \\
5.040 \\
5.000 \\
5.040 \\
5.040 \\
5.040\end{array}$ & $\begin{array}{l}30.151 \\
30.151 \\
30.151 \\
30.131 \\
30.131 \\
30.131 \\
30.171 \\
30.151 \\
30.171 \\
30.171 \\
19.991 \\
20.031 \\
19.971 \\
20.031 \\
20.011 \\
20.011 \\
20.011 \\
19.991 \\
20.031 \\
20.011 \\
10.010 \\
10.015 \\
10.035 \\
10.055 \\
10.035 \\
10.055 \\
10.015 \\
10.055 \\
10.050 \\
10.055 \\
5.026 \\
4.997 \\
4.997 \\
5.037 \\
5.017 \\
5.017 \\
5.012 \\
5.012 \\
4.971 \\
5.012 \\
\end{array}$ \\
\hline
\end{tabular}


Table B.4 Data Set 5 - Absolute Distance Calibration: $10 \mathrm{~m}$ to $150 \mathrm{~m}$

Location: Tunnel, Bldg. 101

No Filter

Test date: April 28, 2001

\begin{tabular}{|c|c|c|c|c|c|c|c|c|c|c|c|c|}
\hline \multirow[b]{3}{*}{$\begin{array}{c}\text { Distance } \\
(\mathrm{m})\end{array}$} & \multirow{2}{*}{\multicolumn{2}{|c|}{ Black }} & \multirow{2}{*}{\multicolumn{2}{|c|}{ White }} & \multirow{2}{*}{\multicolumn{2}{|c|}{ Silver, shiny }} & \multicolumn{6}{|c|}{ 3M Long Distance Performance (LDP) } \\
\hline & & & & & & & \multicolumn{2}{|l|}{$0^{\circ}$} & \multicolumn{2}{|c|}{$45^{\circ}$} & \multicolumn{2}{|l|}{$60^{\circ}$} \\
\hline & $\begin{array}{l}\text { Laser Dist } \\
\text { (m) }\end{array}$ & Inten. & $\begin{array}{l}\text { Laser Dist. } \\
(\mathrm{m})\end{array}$ & Inten. & $\begin{array}{l}\text { Laser Dist. } \\
(\mathrm{m})\end{array}$ & Inten. & $\begin{array}{l}\text { Laser Dist. } \\
\text { (m) }\end{array}$ & Inten. & \begin{tabular}{|c} 
Laser Dist. \\
$(\mathrm{m})$
\end{tabular} & Inten. & \begin{tabular}{|c}
$\begin{array}{c}\text { Laser Dist. } \\
(\mathrm{m})\end{array}$ \\
\end{tabular} & Inten. \\
\hline 9.997 & 10.023 & 80 & 10.007 & 156 & 9.977 & 206 & 10.173 & 228 & 10.009 & 208 & 10.044 & 182 \\
\hline 9.997 & 9.957 & 80 & 9.986 & 156 & 9.972 & 206 & 10.169 & 236 & 9.968 & 208 & 10.024 & 182 \\
\hline 9.997 & 10.018 & 80 & 10.007 & 156 & 9.952 & 206 & 10.109 & 226 & 9.983 & 208 & 9.963 & 182 \\
\hline 9.997 & 9.957 & 80 & 9.946 & 156 & 9.912 & 206 & 10.129 & 228 & 9.983 & 208 & 10.024 & 182 \\
\hline 9.997 & 9.998 & 80 & 9.966 & 156 & 9.972 & 206 & 10.109 & 226 & 9.983 & 208 & 10.044 & 182 \\
\hline 9.997 & 9.998 & 80 & 9.942 & 156 & 10.013 & 204 & 10.149 & 230 & 10.004 & 208 & 9.983 & 182 \\
\hline 9.997 & 9.998 & 80 & 9.982 & 156 & 9.972 & 206 & 10.169 & 230 & 9.943 & 208 & 10.024 & 182 \\
\hline 9.997 & 9.977 & 80 & 10.023 & 156 & 9.972 & 206 & 10.109 & 224 & 10.004 & 208 & 10.024 & 182 \\
\hline 9.997 & 9.988 & 80 & 10.003 & 156 & 9.932 & 204 & 10.189 & 230 & 9.963 & 208 & 10.024 & 182 \\
\hline 9.997 & 10.018 & 80 & 9.982 & 156 & 9.972 & 204 & 10.129 & 228 & 9.943 & 208 & 10.044 & 182 \\
\hline 19.991 & 20.024 & 82 & 19.999 & 152 & 19.999 & 206 & 20.153 & 230 & 20.084 & 190 & 20.144 & 162 \\
\hline 19.991 & 19.984 & 82 & 19.999 & 152 & 19.939 & 206 & 20.108 & 224 & 20.064 & 190 & 20.164 & 162 \\
\hline 19.991 & 20.004 & 84 & 19.959 & 152 & 19.959 & 206 & 20.164 & 232 & 20.064 & 190 & 20.124 & 162 \\
\hline 19.991 & 19.984 & 82 & 19.959 & 152 & 19.939 & 206 & 20.124 & 226 & 20.064 & 190 & 20.164 & 162 \\
\hline 19.991 & 20.004 & 84 & 19.939 & 152 & 19.979 & 206 & 20.124 & 224 & 20.064 & 190 & 20.104 & 162 \\
\hline 19.991 & 20.004 & 82 & 19.979 & 152 & 19.959 & 206 & 20.104 & 224 & 20.064 & 190 & 20.184 & 162 \\
\hline 19.991 & 20.004 & 82 & 19.999 & 152 & 19.959 & 206 & 20.144 & 230 & 20.084 & 190 & 20.124 & 162 \\
\hline 19.991 & 20.004 & 82 & 19.979 & 152 & 19.979 & 206 & 20.164 & 234 & 20.064 & 190 & 20.164 & 162 \\
\hline 19.991 & 19.964 & 82 & 19.959 & 152 & 19.959 & 206 & 20.144 & 230 & 20.064 & 190 & 20.124 & 160 \\
\hline 19.991 & 20.004 & 82 & 19.979 & 152 & 19.979 & 206 & 20.144 & 232 & 20.084 & 190 & 20.164 & 162 \\
\hline 29.986 & 29.914 & 70 & 29.954 & 140 & 29.954 & 192 & 30.074 & 232 & 29.974 & 202 & 30.014 & 172 \\
\hline 29.986 & 29.934 & 70 & 29.934 & 140 & 29.974 & 192 & 30.094 & 224 & 29.954 & 200 & 29.970 & 172 \\
\hline 29.986 & 29.914 & 70 & 29.934 & 140 & 29.974 & 192 & 30.094 & 228 & 29.954 & 200 & 29.974 & 172 \\
\hline 29.986 & 29.894 & 70 & 29.974 & 140 & 29.934 & 192 & 30.054 & 224 & 29.934 & 202 & 29.970 & 172 \\
\hline 29.986 & 29.954 & 70 & 29.974 & 140 & 29.974 & 192 & 30.094 & 224 & 29.930 & 202 & 29.990 & 172 \\
\hline 29.986 & 29.914 & 70 & 29.974 & 140 & 29.974 & 192 & 30.074 & 226 & 29.914 & 200 & 29.990 & 172 \\
\hline 29.986 & 29.934 & 70 & 29.974 & 140 & 29.974 & 192 & 30.074 & 228 & 29.954 & 202 & 29.950 & 172 \\
\hline 29.986 & 29.934 & 70 & 29.934 & 140 & 29.974 & 192 & 30.094 & 232 & 29.914 & 202 & 29.990 & 172 \\
\hline 29.986 & 29.934 & 70 & 29.974 & 140 & 29.954 & 192 & 30.054 & 224 & 29.934 & 200 & 29.994 & 172 \\
\hline 29.986 & 29.934 & 70 & 29.994 & 140 & 29.954 & 192 & 30.094 & 224 & 29.934 & 200 & 29.990 & 172 \\
\hline 39.988 & 39.95 & 62 & 39.97 & 124 & 39.97 & 192 & 40.03 & 226 & 39.970 & 196 & 40.010 & 164 \\
\hline 39.988 & 39.97 & 60 & 39.99 & 124 & 39.93 & 192 & 40.03 & 226 & 39.950 & 196 & 39.965 & 164 \\
\hline 39.988 & 39.93 & 60 & 40.01 & 124 & 39.99 & 192 & 40.05 & 224 & 39.950 & 196 & 39.970 & 164 \\
\hline 39.988 & 39.97 & 62 & 40.01 & 124 & 39.93 & 192 & 40.05 & 224 & 39.950 & 196 & 40.010 & 164 \\
\hline 39.988 & 39.95 & 62 & 39.99 & 124 & 39.99 & 192 & 40.03 & 226 & 39.970 & 196 & 39.970 & 164 \\
\hline 39.988 & 39.95 & 60 & 39.99 & 124 & 39.97 & 192 & 40.03 & 228 & 39.950 & 196 & 40.010 & 164 \\
\hline 39.988 & 39.99 & 60 & 40.01 & 124 & 39.95 & 192 & 40.05 & 222 & 39.970 & 196 & 39.990 & 164 \\
\hline 39.988 & 39.93 & 60 & 39.99 & 124 & 39.99 & 194 & 40.03 & 226 & 39.930 & 196 & 39.990 & 164 \\
\hline 39.988 & 39.93 & 60 & 39.99 & 124 & 39.93 & 192 & 40.05 & 226 & 39.970 & 196 & 40.005 & 164 \\
\hline 39.988 & 39.95 & 60 & 40.01 & 124 & 39.93 & 192 & 40.03 & 222 & 39.970 & 196 & 39.950 & 164 \\
\hline 49.981 & 49.99 & 52 & 49.965 & 112 & 49.945 & 188 & 50.01 & 222 & 49.925 & 190 & 49.945 & 130 \\
\hline 49.981 & 49.965 & 52 & 49.965 & 112 & 49.985 & 188 & 49.985 & 222 & 49.945 & 190 & 49.965 & 130 \\
\hline 49.981 & 49.985 & 52 & 49.985 & 112 & 49.965 & 188 & 50.005 & 222 & 49.945 & 190 & 49.945 & 130 \\
\hline 49.981 & 49.985 & 52 & 50.005 & 112 & 49.925 & 188 & 50.005 & 222 & 49.945 & 190 & 49.950 & 130 \\
\hline 49.981 & 49.925 & 54 & 50.005 & 112 & 49.985 & 188 & 50.005 & 222 & 49.945 & 190 & 49.905 & 130 \\
\hline 49.981 & 50.005 & 54 & 49.965 & 112 & 49.985 & 188 & 49.965 & 222 & 49.905 & 190 & 49.945 & 130 \\
\hline 49.981 & 49.985 & 54 & 49.965 & 112 & 49.985 & 188 & 50.005 & 222 & 49.945 & 190 & 49.945 & 130 \\
\hline 49.981 & 50.025 & 54 & 50.005 & 112 & 49.945 & 188 & 50.005 & 222 & 49.905 & 190 & 49.945 & 130 \\
\hline 49.981 & 49.985 & 52 & 49.965 & 112 & 49.985 & 188 & 49.965 & 222 & 49.945 & 190 & 49.945 & 130 \\
\hline 49.981 & 49.985 & 52 & 50.005 & 112 & 49.945 & 188 & 49.985 & 222 & 49.925 & 190 & 49.905 & 130 \\
\hline 59.977 & 59.99 & 48 & 59.97 & 102 & 59.97 & 182 & 59.99 & 218 & 59.865 & 186 & 59.925 & 146 \\
\hline 59.977 & 60.05 & 48 & 60.01 & 102 & 59.97 & 182 & 59.985 & 220 & 59.885 & 186 & 59.905 & 146 \\
\hline 59.977 & 60.01 & 48 & 59.99 & 102 & 59.95 & 182 & 59.985 & 218 & 59.905 & 188 & 59.865 & 146 \\
\hline 59.977 & 59.95 & 48 & 59.99 & 102 & 59.99 & 182 & 59.985 & 218 & 59.905 & 186 & 59.905 & 146 \\
\hline 59.977 & 59.99 & 48 & 59.95 & 102 & 59.97 & 182 & 59.965 & 218 & 59.865 & 186 & 59.905 & 146 \\
\hline 59.977 & 60.01 & 48 & 60.01 & 102 & 59.93 & 182 & 59.925 & 218 & 59.905 & 186 & 59.925 & 146 \\
\hline 59.977 & 59.95 & 46 & 60.03 & 102 & 59.97 & 182 & 59.945 & 218 & 59.905 & 186 & 59.885 & 146 \\
\hline
\end{tabular}


Table B.4 Data Set 5 - Absolute Distance Calibration: $10 \mathrm{~m}$ to $150 \mathrm{~m}$

\begin{tabular}{|c|c|c|c|c|c|c|c|c|c|c|c|c|}
\hline \multicolumn{13}{|c|}{$\begin{array}{l}\text { Location: Tunnel, Bldg. } 101 \\
\text { No Filter } \\
\text { Test date: April 28, } 2001\end{array}$} \\
\hline \multirow[b]{3}{*}{$\begin{array}{c}\text { Distance } \\
(\mathrm{m})\end{array}$} & \multirow{2}{*}{\multicolumn{2}{|c|}{ Black }} & \multirow{2}{*}{\multicolumn{2}{|c|}{ White }} & \multirow{2}{*}{\multicolumn{2}{|c|}{ Silver, shiny }} & \multicolumn{6}{|c|}{ 3M Long Distance Performance (LDP) } \\
\hline & & & & & & & \multicolumn{2}{|l|}{$0^{\circ}$} & \multicolumn{2}{|c|}{$45^{\circ}$} & \multicolumn{2}{|l|}{$60^{\circ}$} \\
\hline & $\begin{array}{l}\text { Laser Dist } \\
(\mathrm{m})\end{array}$ & Inten. & $\begin{array}{l}\text { Laser Dist. } \\
(\mathrm{m})\end{array}$ & Inten. & $\begin{array}{l}\text { Laser Dist. } \\
\text { (m) }\end{array}$ & Inten. & \begin{tabular}{|c}
$\begin{array}{c}\text { Laser Dist. } \\
(\mathrm{m})\end{array}$ \\
\end{tabular} & Inten. & \begin{tabular}{|c} 
Laser Dist. \\
(m)
\end{tabular} & Inten. & \begin{tabular}{|c} 
Laser Dist. \\
$(\mathrm{m})$
\end{tabular} & Inten. \\
\hline 59.977 & 59.99 & 48 & 60.01 & 102 & 59.97 & 182 & 59.985 & 220 & 59.905 & 186 & 59.865 & 146 \\
\hline 59.977 & 59.99 & 48 & 59.99 & 102 & 59.97 & 182 & 59.965 & 218 & 59.905 & 186 & 59.905 & 146 \\
\hline 59.977 & 59.99 & 48 & 59.97 & 102 & 59.97 & 182 & 59.985 & 218 & 59.905 & 186 & 59.905 & 146 \\
\hline 69.971 & 69.985 & 42 & 69.985 & 94 & 69.945 & 178 & 69.965 & 216 & 69.945 & 186 & 69.925 & 140 \\
\hline 69.971 & 69.985 & 42 & 69.965 & 94 & 69.985 & 178 & 69.925 & 216 & 69.945 & 186 & 69.885 & 138 \\
\hline 69.971 & 70.005 & 42 & 69.965 & 94 & 69.965 & 178 & 69.985 & 216 & 69.945 & 186 & 69.945 & 140 \\
\hline 69.971 & 69.985 & 42 & 69.985 & 94 & 69.965 & 178 & 69.965 & 216 & 69.925 & 186 & 69.945 & 140 \\
\hline 69.971 & 69.985 & 42 & 69.985 & 94 & 69.965 & 178 & 69.925 & 216 & 69.945 & 186 & 69.925 & 140 \\
\hline 69.971 & 69.985 & 42 & 69.965 & 94 & 69.925 & 178 & 69.965 & 216 & 69.945 & 186 & 69.925 & 138 \\
\hline 69.971 & 70.025 & 42 & 69.965 & 94 & 69.945 & 178 & 69.965 & 216 & 69.945 & 186 & 69.885 & 140 \\
\hline 69.971 & 69.945 & 42 & 69.985 & 94 & 69.965 & 178 & 69.985 & 216 & 69.905 & 186 & 69.905 & 140 \\
\hline 69.971 & 69.945 & 44 & 70.005 & 94 & 69.965 & 178 & 69.965 & 216 & 69.925 & 184 & 69.905 & 140 \\
\hline 69.971 & 69.985 & 42 & 69.985 & 94 & 69.965 & 178 & 69.945 & 216 & 69.905 & 186 & 69.925 & 140 \\
\hline 79.967 & 79.985 & 40 & 79.965 & 86 & 79.965 & 182 & 79.965 & 214 & 79.865 & 184 & 79.925 & 120 \\
\hline 79.967 & 80.025 & 38 & 79.965 & 86 & 79.965 & 184 & 79.945 & 214 & 79.845 & 184 & 79.885 & 120 \\
\hline 79.967 & 79.925 & 40 & 79.985 & 88 & 79.885 & 182 & 79.945 & 214 & 79.845 & 184 & 79.905 & 122 \\
\hline 79.967 & 80.005 & 40 & 79.985 & 86 & 79.945 & 182 & 79.965 & 214 & 79.885 & 184 & 79.945 & 122 \\
\hline 79.967 & 79.945 & 40 & 79.965 & 86 & 79.965 & 184 & 79.965 & 214 & 79.885 & 184 & 79.945 & 120 \\
\hline 79.967 & 79.965 & 40 & 80.025 & 88 & 79.965 & 182 & 79.905 & 214 & 79.905 & 184 & 79.925 & 120 \\
\hline 79.967 & 80.005 & 38 & 80.025 & 88 & 79.905 & 182 & 79.965 & 214 & 79.845 & 184 & 79.925 & 122 \\
\hline 79.967 & 79.965 & 40 & 79.965 & 86 & 79.965 & 182 & 79.945 & 214 & 79.865 & 184 & 79.945 & 122 \\
\hline 79.967 & 79.985 & 38 & 79.965 & 86 & 79.945 & 182 & 79.965 & 214 & 79.865 & 184 & 79.885 & 120 \\
\hline 79.967 & 80.025 & 40 & 79.985 & 88 & 79.945 & 182 & 79.985 & 214 & 79.905 & 184 & 79.945 & 122 \\
\hline 89.961 & 89.973 & 38 & 89.982 & 84 & 89.942 & 184 & 89.893 & 212 & 89.868 & 184 & 89.928 & 112 \\
\hline 89.961 & 89.938 & 38 & 89.958 & 84 & 89.922 & 182 & 89.893 & 214 & 89.828 & 184 & 89.988 & 112 \\
\hline 89.961 & 89.993 & 38 & 89.938 & 84 & 89.962 & 182 & 89.888 & 214 & 89.828 & 184 & 89.968 & 112 \\
\hline 89.961 & 89.953 & 38 & 89.998 & 84 & 89.942 & 182 & 89.933 & 214 & 89.868 & 184 & 89.968 & 112 \\
\hline 89.961 & 89.933 & 38 & 89.978 & 84 & 89.902 & 182 & 89.933 & 214 & 89.888 & 184 & 89.968 & 112 \\
\hline 89.961 & 89.973 & 40 & 89.958 & 84 & 89.942 & 182 & 89.908 & 214 & 89.868 & 184 & 89.968 & 112 \\
\hline 89.961 & 89.953 & 38 & 89.978 & 84 & 89.922 & 184 & 89.933 & 214 & 89.868 & 184 & 89.968 & 112 \\
\hline 89.961 & 89.973 & 38 & 89.978 & 84 & 89.942 & 182 & 89.948 & 214 & 89.848 & 184 & 89.968 & 112 \\
\hline 89.961 & 89.993 & 38 & 89.978 & 84 & 89.942 & 184 & 89.948 & 214 & 89.828 & 184 & 89.968 & 112 \\
\hline 89.961 & 89.953 & 38 & 89.958 & 84 & 89.902 & 184 & 89.888 & 214 & 89.868 & 184 & 89.988 & 112 \\
\hline 99.955 & 99.964 & 34 & 99.919 & 78 & 99.944 & 184 & 99.928 & 210 & 99.864 & 182 & 99.964 & 102 \\
\hline 99.955 & 99.984 & 34 & 99.979 & 78 & 99.939 & 184 & 99.924 & 210 & 99.864 & 182 & 99.964 & 102 \\
\hline 99.955 & 99.944 & 36 & 99.959 & 78 & 99.939 & 184 & 99.884 & 210 & 99.864 & 182 & 99.924 & 102 \\
\hline 99.955 & 99.964 & 36 & 99.959 & 78 & 99.959 & 184 & 99.924 & 212 & 99.864 & 182 & 99.984 & 102 \\
\hline 99.955 & 100.004 & 36 & 99.959 & 78 & 99.919 & 184 & 99.884 & 210 & 99.844 & 182 & 99.984 & 102 \\
\hline 99.955 & 99.964 & 36 & 99.919 & 78 & 99.939 & 184 & 99.904 & 210 & 99.864 & 182 & 99.964 & 102 \\
\hline 99.955 & 99.924 & 36 & 99.959 & 80 & 99.959 & 184 & 99.944 & 210 & 99.864 & 182 & 99.964 & 102 \\
\hline 99.955 & 100.004 & 36 & 99.959 & 78 & 99.939 & 184 & 99.944 & 210 & 99.864 & 182 & 99.964 & 102 \\
\hline 99.955 & 100.044 & 36 & 99.959 & 78 & 99.939 & 184 & 99.924 & 210 & 99.864 & 182 & 99.984 & 102 \\
\hline 99.955 & 99.984 & 36 & 99.959 & 78 & 99.899 & 184 & 99.884 & 210 & 99.824 & 182 & 99.944 & 102 \\
\hline 109.949 & 109.959 & 34 & 109.939 & 74 & 109.954 & 176 & 109.914 & 208 & 109.854 & 180 & 109.914 & 98 \\
\hline 109.949 & 109.879 & 34 & 109.934 & 74 & 109.874 & 174 & 109.984 & 208 & 109.854 & 180 & 109.934 & 98 \\
\hline 109.949 & 109.979 & 34 & 109.934 & 76 & 109.914 & 174 & 109.934 & 208 & 109.874 & 180 & 109.954 & 98 \\
\hline 109.949 & 109.914 & 34 & 109.934 & 76 & 109.954 & 174 & 109.914 & 208 & 109.834 & 180 & 109.954 & 98 \\
\hline 109.949 & 109.919 & 34 & 109.934 & 74 & 109.894 & 176 & 109.934 & 208 & 109.854 & 180 & 109.954 & 98 \\
\hline 109.949 & 109.854 & 34 & 109.934 & 76 & 109.934 & 174 & 109.914 & 208 & 109.874 & 180 & 109.974 & 98 \\
\hline 109.949 & 109.959 & 34 & 109.894 & 74 & 109.934 & 174 & 109.934 & 208 & 109.814 & 180 & 109.974 & 98 \\
\hline 109.949 & 109.954 & 34 & 109.954 & 74 & 109.894 & 174 & 109.934 & 208 & 109.854 & 180 & 109.954 & 98 \\
\hline 109.949 & 109.959 & 34 & 109.934 & 76 & 109.914 & 176 & 109.934 & 208 & 109.874 & 180 & 109.974 & 98 \\
\hline 109.949 & 109.974 & 34 & 109.914 & 74 & 109.934 & 174 & 109.914 & 208 & 109.874 & 180 & 109.954 & 98 \\
\hline 119.944 & 119.93 & 34 & 119.914 & 72 & 119.934 & 170 & 119.914 & 206 & 119.874 & 170 & 119.934 & 88 \\
\hline 119.944 & 119.99 & 34 & 119.874 & 72 & 119.934 & 174 & 119.934 & 206 & 119.894 & 170 & 119.954 & 88 \\
\hline 119.944 & 119.95 & 34 & 119.93 & 72 & 119.934 & 168 & 119.934 & 206 & 119.874 & 172 & 119.934 & 88 \\
\hline 119.944 & 119.87 & 34 & 119.93 & 72 & 119.934 & 170 & 119.934 & 206 & 119.954 & 170 & 119.954 & 88 \\
\hline 119.944 & 119.93 & 34 & 119.89 & 72 & 119.914 & 168 & 119.934 & 206 & 119.984 & 170 & 119.914 & 88 \\
\hline
\end{tabular}


Table B.4 Data Set 5 - Absolute Distance Calibration: $10 \mathrm{~m}$ to $150 \mathrm{~m}$

\begin{tabular}{|c|c|c|c|c|c|c|c|c|c|c|c|c|}
\hline \multicolumn{13}{|c|}{$\begin{array}{l}\text { Location: Tunnel, Bldg. } 101 \\
\text { No Filter } \\
\text { Test date: April 28, } 2001 \\
\end{array}$} \\
\hline \multirow[b]{3}{*}{$\begin{array}{c}\text { Distance } \\
(\mathrm{m})\end{array}$} & \multirow{2}{*}{\multicolumn{2}{|c|}{ Black }} & \multirow{2}{*}{\multicolumn{2}{|c|}{ White }} & \multirow{2}{*}{\multicolumn{2}{|c|}{ Silver, shiny }} & \multicolumn{6}{|c|}{ 3M Long Distance Performance (LDP) } \\
\hline & & & & & & & \multicolumn{2}{|l|}{$0^{\circ}$} & \multicolumn{2}{|l|}{$45^{\circ}$} & \multicolumn{2}{|l|}{$60^{\circ}$} \\
\hline & $\begin{array}{l}\text { Laser Dist } \\
(\mathrm{m})\end{array}$ & Inten. & $\begin{array}{c}\text { Laser Dist. } \\
(\mathrm{m})\end{array}$ & Inten. & $\begin{array}{l}\text { Laser Dist. } \\
(\mathrm{m})\end{array}$ & Inten. & \begin{tabular}{|c} 
Laser Dist. \\
$(\mathrm{m})$
\end{tabular} & Inten. & \begin{tabular}{|c} 
Laser Dist. \\
(m)
\end{tabular} & Inten. & \begin{tabular}{|c|}
$\begin{array}{c}\text { Laser Dist. } \\
(\mathrm{m})\end{array}$ \\
\end{tabular} & Inten. \\
\hline 119.944 & 119.97 & 34 & 119.91 & 72 & 119.934 & 168 & 119.914 & 206 & 119.894 & 170 & \begin{tabular}{|l|}
119.914 \\
\end{tabular} & 88 \\
\hline 119.944 & 119.93 & 34 & 119.91 & 72 & 119.93 & 168 & 119.934 & 206 & 119.894 & 170 & 119.954 & 88 \\
\hline 119.944 & 119.99 & 34 & 119.93 & 72 & 119.95 & 168 & 119.934 & 206 & 119.894 & 170 & 119.950 & 88 \\
\hline 119.944 & 119.91 & 34 & 119.87 & 72 & 119.914 & 168 & 119.914 & 206 & 119.874 & 172 & 119.934 & 88 \\
\hline 119.944 & 119.89 & 34 & 119.874 & 72 & 119.95 & 168 & 119.894 & 206 & 119.854 & 170 & 119.954 & 88 \\
\hline 129.941 & 129.91 & 36 & 129.91 & 68 & 129.93 & 166 & 129.91 & 204 & 129.910 & 162 & 129.890 & 80 \\
\hline 129.941 & 129.91 & 36 & 129.89 & 68 & 129.91 & 166 & 129.91 & 204 & 129.930 & 162 & 129.890 & 80 \\
\hline 129.941 & 129.97 & 36 & 129.89 & 68 & 129.95 & 166 & 129.89 & 204 & 129.910 & 162 & 129.910 & 80 \\
\hline 129.941 & 129.93 & 36 & 129.91 & 68 & 129.89 & 166 & 129.87 & 204 & 129.910 & 162 & 129.910 & 80 \\
\hline 129.941 & 129.93 & 36 & 129.91 & 68 & 129.95 & 166 & 129.93 & 204 & 129.910 & 162 & 129.910 & 80 \\
\hline 129.941 & 129.95 & 36 & 129.89 & 68 & 129.93 & 166 & 129.93 & 204 & 129.950 & 162 & 129.910 & 80 \\
\hline 129.941 & 129.95 & 36 & 129.93 & 68 & 129.93 & 164 & 129.93 & 204 & 129.910 & 162 & 129.870 & 80 \\
\hline 129.941 & 129.95 & 36 & 129.91 & 68 & 129.91 & 166 & 129.93 & 204 & 129.890 & 162 & 129.930 & 80 \\
\hline 129.941 & 129.87 & 36 & 129.87 & 68 & 129.91 & 166 & 129.93 & 204 & 129.930 & 162 & 129.890 & 80 \\
\hline 129.941 & 129.91 & 36 & 129.91 & 68 & 129.91 & 166 & 129.91 & 204 & 129.890 & 162 & 129.910 & 80 \\
\hline 139.939 & 140.048 & 44 & 139.928 & 68 & 139.924 & 156 & 139.898 & 204 & 139.873 & 154 & 139.968 & 82 \\
\hline 139.939 & 139.968 & 46 & 139.884 & 68 & 139.884 & 158 & 139.893 & 204 & 139.873 & 154 & 139.908 & 82 \\
\hline 139.939 & 139.988 & 44 & 139.864 & 68 & 139.944 & 162 & 139.913 & 204 & 139.873 & 154 & 139.928 & 82 \\
\hline 139.939 & 140.008 & 44 & 139.904 & 68 & 139.944 & 162 & 139.893 & 204 & 139.893 & 154 & 139.928 & 82 \\
\hline 139.939 & 139.928 & 44 & 139.904 & 68 & 139.944 & 162 & 139.893 & 204 & 139.873 & 154 & 139.928 & 82 \\
\hline 139.939 & 139.968 & 46 & 139.904 & 68 & 139.944 & 164 & 139.913 & 204 & 139.893 & 154 & 139.928 & 82 \\
\hline 139.939 & 140.028 & 44 & 139.904 & 68 & 139.944 & 162 & 139.933 & 204 & 139.933 & 154 & 139.908 & 82 \\
\hline 139.939 & 139.968 & 44 & 139.904 & 68 & 139.944 & 164 & 139.893 & 204 & 139.913 & 154 & 139.928 & 82 \\
\hline 139.939 & 139.988 & 44 & 139.864 & 68 & 139.944 & 162 & 139.893 & 204 & 139.893 & 154 & 139.908 & 82 \\
\hline 139.939 & 139.928 & 44 & 139.904 & 68 & 139.944 & 162 & 139.913 & 204 & 139.893 & 154 & 139.948 & 82 \\
\hline 149.930 & 149.87 & 64 & 149.79 & 76 & 149.91 & 152 & 149.89 & 200 & 149.91 & 152 & 150.070 & 92 \\
\hline 149.930 & 149.89 & 64 & 149.83 & 76 & 149.91 & 152 & 149.89 & 200 & 149.89 & 152 & 150.110 & 92 \\
\hline 149.930 & 149.93 & 62 & 149.81 & 76 & 149.87 & 152 & 149.89 & 200 & 149.89 & 152 & 150.090 & 92 \\
\hline 149.930 & 149.89 & 64 & 149.77 & 76 & 149.87 & 152 & 149.91 & 200 & 149.89 & 152 & 150.070 & 92 \\
\hline 149.930 & 149.85 & 62 & 149.81 & 76 & 149.91 & 152 & 149.89 & 200 & 149.91 & 152 & 150.070 & 92 \\
\hline 149.930 & 149.89 & 64 & 149.81 & 76 & 149.89 & 152 & 149.89 & 200 & 149.91 & 152 & 150.070 & 92 \\
\hline 149.930 & 149.85 & 64 & 149.81 & 76 & 149.89 & 152 & 149.87 & 200 & 149.89 & 152 & 150.090 & 92 \\
\hline 149.930 & 149.83 & 64 & 149.79 & 76 & 149.93 & 152 & 149.89 & 200 & 149.91 & 152 & 150.070 & 92 \\
\hline 149.930 & 149.95 & 62 & 149.77 & 76 & 149.89 & 158 & 149.85 & 200 & 149.97 & 152 & 150.090 & 92 \\
\hline 149.930 & 149.87 & 62 & 149.85 & 76 & 149.91 & 152 & 149.85 & 200 & 149.91 & 152 & 150.050 & 92 \\
\hline 149.930 & 149.95 & 62 & 149.81 & 76 & 149.91 & 158 & 149.89 & 200 & 149.87 & 152 & 150.090 & 92 \\
\hline
\end{tabular}


Table B.5 Data Set 6 - Angle of Laser Incidence: $10 \mathrm{~m}$ to $100 \mathrm{~m}$

Attic, Bldg. 226

No Filter

Test Dates: Nov. 1, 5, and 28, 2001

File: rot_calib_11_01_01.xls

\begin{tabular}{|c|c|c|c|c|c|c|c|c|c|c|c|c|}
\hline \multirow{4}{*}{$\begin{array}{c}\text { Distance } \\
(\mathrm{m})\end{array}$} & \multicolumn{12}{|c|}{ Silver, shiny } \\
\hline & \multicolumn{12}{|c|}{ Rotation Angle $\left({ }^{\circ}\right)$} \\
\hline & \multicolumn{2}{|c|}{0} & \multicolumn{2}{|c|}{10} & \multicolumn{2}{|c|}{20} & \multicolumn{2}{|c|}{30} & \multicolumn{2}{|c|}{40} & \multicolumn{2}{|c|}{50} \\
\hline & Dist. (m) & Inten. & Dist. (m) & Inten. & Dist. (m) & Inten. & Dist. (m) & Inten. & Dist. (m) & Inten. & Dist. (m) & Inten \\
\hline 10 & 9.947 & 200 & 9.987 & 170 & 10.028 & 126 & 10.128 & 98 & 10.268 & 84 & 10.688 & 86 \\
\hline 10 & 9.987 & 200 & 10.008 & 170 & 10.028 & 126 & 10.068 & 98 & 10.268 & 86 & 10.668 & 86 \\
\hline 10 & 9.987 & 200 & 10.008 & 170 & 10.028 & 126 & 10.068 & 98 & 10.248 & 84 & 10.668 & 86 \\
\hline 10 & 9.927 & 200 & 9.947 & 170 & 10.028 & 126 & 10.068 & 98 & 10.288 & 86 & 10.668 & 86 \\
\hline 10 & 9.947 & 200 & 9.947 & 170 & 9.987 & 126 & 10.108 & 98 & 10.268 & 84 & 10.688 & 86 \\
\hline 10 & 9.947 & 200 & 10.008 & 170 & 9.987 & 126 & 10.068 & 98 & 10.248 & 86 & 10.668 & 86 \\
\hline 10 & 9.987 & 200 & 9.987 & 170 & 10.028 & 126 & 10.108 & 98 & 10.228 & 84 & 10.668 & 86 \\
\hline 10 & 9.967 & 200 & 10.008 & 170 & 10.028 & 126 & 10.088 & 98 & 10.248 & 86 & 10.648 & 86 \\
\hline 10 & 9.987 & 200 & 10.008 & 170 & 10.028 & 126 & 10.088 & 98 & 10.288 & 84 & 10.628 & 86 \\
\hline 10 & 10.008 & 200 & 9.987 & 170 & 9.987 & 126 & 10.108 & 98 & 10.288 & 84 & 10.688 & 86 \\
\hline 20 & 19.962 & 200 & 20.002 & 176 & 19.982 & 132 & 20.022 & 98 & 19.982 & 80 & 19.862 & 72 \\
\hline 20 & 19.982 & 200 & 20.002 & 176 & 19.982 & 132 & 20.062 & 98 & 19.982 & 80 & 19.842 & 72 \\
\hline 20 & 19.982 & 200 & 19.982 & 176 & 19.962 & 130 & 20.022 & 98 & 20.002 & 80 & 19.842 & 72 \\
\hline 20 & 19.922 & 200 & 20.002 & 176 & 19.962 & 132 & 20.062 & 98 & 20.022 & 80 & 19.902 & 72 \\
\hline 20 & 19.962 & 200 & 20.002 & 176 & 20.002 & 130 & 20.002 & 98 & 20.022 & 80 & 19.842 & 72 \\
\hline 20 & 19.982 & 202 & 20.002 & 176 & 19.982 & 132 & 20.042 & 98 & 20.042 & 80 & 19.822 & 74 \\
\hline 20 & 19.982 & 200 & 19.982 & 176 & 20.002 & 130 & 20.022 & 98 & 20.022 & 80 & 19.822 & 74 \\
\hline 20 & 19.982 & 200 & 20.002 & 176 & 19.982 & 130 & 20.062 & 98 & 20.002 & 80 & 19.842 & 74 \\
\hline 20 & 19.982 & 200 & 19.962 & 176 & 20.002 & 132 & 20.002 & 98 & 20.002 & 80 & 19.802 & 74 \\
\hline 20 & 19.922 & 200 & 19.982 & 176 & 20.022 & 132 & 20.042 & 98 & 20.022 & 80 & 19.862 & 72 \\
\hline 30 & 29.96 & 190 & 29.96 & 150 & 30.06 & 106 & 30.06 & 80 & 30.38 & 78 & 30.6 & 76 \\
\hline 30 & 29.98 & 190 & 29.98 & 148 & 30.04 & 106 & 30.08 & 80 & 30.4 & 78 & 30.48 & 66 \\
\hline 30 & 29.96 & 190 & 29.98 & 150 & 30.06 & 106 & 30.12 & 80 & 30.42 & 78 & 30.34 & 58 \\
\hline 30 & 29.98 & 190 & 29.94 & 150 & 30.04 & 106 & 30.08 & 80 & 30.42 & 78 & 30.36 & 60 \\
\hline 30 & 29.98 & 190 & 29.94 & 150 & 30.06 & 106 & 30.1 & 80 & 30.42 & 78 & 30.36 & 58 \\
\hline 30 & 29.98 & 190 & 29.98 & 150 & 30.02 & 106 & 30.1 & 80 & 30.4 & 78 & 30.36 & 58 \\
\hline 30 & 29.96 & 190 & 29.98 & 150 & 30.06 & 106 & 30.1 & 80 & 30.38 & 78 & 30.34 & 58 \\
\hline 30 & 29.96 & 190 & 29.94 & 150 & 30.06 & 106 & 30.12 & 80 & 30.44 & 78 & 30.32 & 58 \\
\hline 30 & 29.92 & 190 & 29.98 & 150 & 30.06 & 106 & 30.08 & 80 & 30.52 & 78 & 30.34 & 58 \\
\hline 30 & 29.94 & 190 & 29.98 & 150 & 30.06 & 106 & 30.08 & 80 & 30.52 & 80 & 30.36 & 58 \\
\hline 40 & 39.94 & 192 & 39.98 & 148 & 39.996 & 96 & 39.916 & 72 & 40.556 & 80 & 41.016 & 58 \\
\hline 40 & 39.98 & 190 & 39.976 & 148 & 39.976 & 96 & 39.916 & 72 & 40.416 & 68 & 41.076 & 58 \\
\hline 40 & 39.94 & 190 & 39.936 & 148 & 39.996 & 96 & 39.956 & 72 & 39.936 & 60 & 41.016 & 58 \\
\hline 40 & 39.94 & 190 & 39.976 & 148 & 39.996 & 96 & 39.936 & 72 & 39.956 & 60 & 40.976 & 58 \\
\hline 40 & 39.96 & 192 & 39.956 & 148 & 40.016 & 96 & 39.956 & 72 & 39.996 & 62 & 41.076 & 58 \\
\hline 40 & 39.96 & 190 & 39.996 & 148 & 39.976 & 96 & 39.916 & 72 & 39.936 & 62 & 41.016 & 58 \\
\hline 40 & 39.94 & 190 & 39.976 & 148 & 39.976 & 94 & 39.916 & 72 & 39.976 & 62 & 41.056 & 58 \\
\hline 40 & 39.94 & 192 & 39.936 & 148 & 39.976 & 94 & 39.956 & 72 & 39.936 & 62 & 40.616 & 62 \\
\hline 40 & 39.94 & 190 & 39.976 & 148 & 40.016 & 96 & 39.916 & 72 & 39.976 & 62 & 40.996 & 58 \\
\hline 40 & 39.94 & 192 & 39.956 & 148 & 39.996 & 94 & 39.956 & 72 & 39.956 & 62 & 40.996 & 58 \\
\hline 50 & 49.922 & 184 & 50.002 & 142 & 50.042 & 96 & 50.302 & 72 & 50.622 & 62 & 51.102 & 64 \\
\hline 50 & 49.962 & 184 & 49.962 & 140 & 50.022 & 94 & 50.322 & 72 & 50.662 & 62 & 51.102 & 64 \\
\hline 50 & 49.942 & 184 & 49.982 & 140 & 50.022 & 94 & 50.262 & 72 & 50.662 & 62 & 51.102 & 66 \\
\hline 50 & 49.942 & 184 & 49.942 & 140 & 50.042 & 94 & 50.322 & 72 & 50.662 & 62 & 51.062 & 66 \\
\hline 50 & 49.922 & 184 & 49.962 & 140 & 50.042 & 94 & 50.302 & 72 & 50.642 & 62 & 51.062 & 66 \\
\hline 50 & 49.942 & 184 & 49.962 & 140 & 50.042 & 94 & 50.302 & 72 & 50.622 & 62 & 51.082 & 64 \\
\hline 50 & 49.942 & 184 & 49.982 & 140 & 50.042 & 96 & 50.262 & 72 & 50.642 & 62 & 51.062 & 64 \\
\hline 50 & 49.942 & 184 & 49.962 & 140 & 50.022 & 94 & 50.282 & 72 & 50.642 & 62 & 51.042 & 66 \\
\hline 50 & 49.942 & 184 & 49.982 & 140 & 50.022 & 94 & 50.302 & 72 & 50.622 & 62 & 51.082 & 66 \\
\hline 50 & 49.942 & 184 & 49.982 & 140 & 50.022 & 94 & 50.322 & 72 & 50.662 & 62 & 51.062 & 66 \\
\hline 60 & 59.968 & 184 & 59.984 & 134 & 60.019 & 84 & 60.094 & 62 & 60.47 & 60 & 60.685 & 70 \\
\hline 60 & 59.908 & 184 & 59.959 & 136 & 60.034 & 84 & 60.05 & 62 & 60.49 & 60 & 60.725 & 70 \\
\hline 60 & 59.908 & 184 & 59.919 & 134 & 59.994 & 84 & 60.09 & 62 & 60.49 & 60 & 60.745 & 70 \\
\hline 60 & 59.948 & 184 & 59.939 & 136 & 60.014 & 84 & 60.07 & 64 & 60.485 & 60 & 60.725 & 70 \\
\hline 60 & 59.928 & 184 & 59.919 & 136 & 60.034 & 84 & 60.11 & 62 & 60.51 & 60 & 60.745 & 70 \\
\hline 60 & 59.988 & 184 & 59.919 & 136 & 59.994 & 84 & 60.09 & 64 & 60.53 & 60 & 60.745 & 68 \\
\hline
\end{tabular}


Table B.5 Data Set 6 - Angle of Laser Incidence: $10 \mathrm{~m}$ to $100 \mathrm{~m}$

\begin{tabular}{|c|c|c|c|c|c|c|c|c|c|c|c|c|}
\hline \multicolumn{13}{|c|}{$\begin{array}{l}\text { Attic, Bldg. } 226 \\
\text { No Filter } \\
\text { Test Dates: Nov. 1, 5, and 28, } 2001\end{array}$} \\
\hline \multirow{4}{*}{$\begin{array}{c}\text { Distance } \\
(\mathrm{m})\end{array}$} & \multicolumn{12}{|c|}{ Silver, shiny } \\
\hline & \multicolumn{12}{|c|}{ Rotation Angle $\left({ }^{\circ}\right)$} \\
\hline & \multicolumn{2}{|c|}{0} & \multicolumn{2}{|c|}{10} & \multicolumn{2}{|c|}{20} & \multicolumn{2}{|c|}{30} & \multicolumn{2}{|c|}{40} & \multicolumn{2}{|c|}{50} \\
\hline & Dist. (m) & Inten. & Dist. (m) & Inten. & Dist. (m) & Inten. & Dist. (m) & Inten. & Dist. (m) & Inten. & Dist. (m) & Inten. \\
\hline 60 & 59.964 & 184 & 59.979 & 134 & 60.034 & 84 & 60.09 & 62 & 60.49 & 60 & 60.765 & 68 \\
\hline 60 & 59.944 & 184 & 59.939 & 134 & 60.034 & 82 & 60.07 & 62 & 60.51 & 60 & 60.765 & 68 \\
\hline 60 & 59.944 & 184 & 59.919 & 134 & 60.034 & 84 & 60.09 & 62 & 60.485 & 60 & 60.725 & 68 \\
\hline 60 & 59.904 & 184 & 59.919 & 134 & 60.034 & 84 & 60.07 & 64 & 60.49 & 60 & 60.765 & 68 \\
\hline 70 & 69.922 & 182 & 69.982 & 112 & 70.042 & 68 & 70.282 & 52 & 70.962 & 52 & 71.082 & 48 \\
\hline 70 & 69.962 & 182 & 70.002 & 112 & 70.022 & 70 & 70.222 & 52 & 71.062 & 52 & 71.082 & 48 \\
\hline 70 & 69.942 & 182 & 70.002 & 112 & 70.002 & 68 & 70.282 & 52 & 70.922 & 52 & 71.082 & 48 \\
\hline 70 & 69.942 & 182 & 70.002 & 112 & 70.022 & 70 & 70.262 & 52 & 70.962 & 52 & 71.082 & 48 \\
\hline 70 & 69.942 & 182 & 70.002 & 112 & 70.022 & 70 & 70.282 & 52 & 71.002 & 52 & 71.062 & 48 \\
\hline 70 & 69.942 & 182 & 69.982 & 112 & 69.982 & 70 & 70.282 & 52 & 71.002 & 52 & 71.122 & 48 \\
\hline 70 & 69.922 & 182 & 69.962 & 112 & 69.982 & 70 & 70.302 & 52 & 70.982 & 52 & 71.042 & 48 \\
\hline 70 & 69.942 & 182 & 69.962 & 112 & 70.002 & 70 & 70.262 & 52 & 71.002 & 52 & 71.062 & 48 \\
\hline 70 & 69.922 & 182 & 70.002 & 112 & 70.022 & 70 & 70.302 & 52 & 71.002 & 52 & 71.102 & 48 \\
\hline 70 & 69.942 & 182 & 69.982 & 112 & 69.982 & 68 & 70.282 & 52 & 71.022 & 52 & 71.102 & 48 \\
\hline 80 & 79.937 & 172 & 79.972 & 96 & 79.932 & 62 & 79.992 & 44 & 79.997 & 44 & 79.957 & 32 \\
\hline 80 & 79.977 & 172 & 79.977 & 96 & 79.937 & 62 & 79.977 & 44 & 80.277 & 40 & 80.037 & 34 \\
\hline 80 & 79.972 & 172 & 79.997 & 96 & 79.952 & 62 & 79.997 & 44 & 80.057 & 38 & 80.097 & 34 \\
\hline 80 & 79.952 & 172 & 79.957 & 96 & 79.957 & 62 & 79.907 & 44 & 80.077 & 40 & 79.997 & 32 \\
\hline 80 & 79.957 & 172 & 79.992 & 96 & 79.932 & 62 & 80.077 & 44 & 79.977 & 38 & 80.137 & 34 \\
\hline 80 & 79.952 & 172 & 79.997 & 96 & 79.937 & 62 & 79.977 & 44 & 80.217 & 40 & 79.957 & 32 \\
\hline 80 & 79.957 & 172 & 79.977 & 96 & 79.957 & 62 & 80.017 & 44 & 80.057 & 40 & 79.957 & 32 \\
\hline 80 & 79.977 & 172 & 79.977 & 96 & 79.937 & 62 & 79.997 & 44 & 80.077 & 40 & 79.957 & 32 \\
\hline 80 & 79.977 & 172 & 79.957 & 96 & 79.932 & 62 & 80.017 & 44 & 79.997 & 38 & 80.017 & 32 \\
\hline 80 & 79.972 & 172 & 79.997 & 96 & 79.977 & 62 & 79.997 & 44 & 80.037 & 40 & 79.957 & 32 \\
\hline 90 & 89.937 & 174 & 89.997 & 102 & 89.957 & 60 & 90.017 & 42 & 90.037 & 42 & 91.057 & 38 \\
\hline 90 & 89.937 & 174 & 89.997 & 102 & 89.977 & 60 & 90.057 & 42 & 90.597 & 42 & 91.117 & 38 \\
\hline 90 & 89.937 & 174 & 89.977 & 102 & 89.977 & 60 & 89.977 & 42 & 90.537 & 38 & 91.017 & 38 \\
\hline 90 & 89.897 & 174 & 89.977 & 102 & 89.977 & 60 & 89.997 & 42 & 90.537 & 42 & 91.057 & 38 \\
\hline 90 & 89.937 & 174 & 89.997 & 102 & 89.957 & 60 & 90.037 & 42 & 90.217 & 38 & 90.977 & 38 \\
\hline 90 & 89.917 & 174 & 89.977 & 102 & 89.957 & 60 & 90.057 & 42 & 90.577 & 42 & 91.057 & 36 \\
\hline 90 & 89.937 & 174 & 89.977 & 102 & 89.937 & 60 & 89.997 & 42 & 89.977 & 38 & 91.017 & 38 \\
\hline 90 & 89.937 & 174 & 89.997 & 102 & 89.957 & 60 & 89.977 & 42 & 90.037 & 38 & 91.017 & 38 \\
\hline 90 & 89.937 & 174 & 89.957 & 102 & 89.937 & 60 & 90.017 & 42 & 90.017 & 36 & 91.017 & 38 \\
\hline 90 & 89.917 & 174 & 90.017 & 102 & 89.977 & 60 & 90.037 & 42 & 90.037 & 38 & 91.017 & 38 \\
\hline 100 & 99.937 & 176 & 99.997 & 92 & 100.137 & 42 & 100.117 & 38 & 99.997 & 44 & 100.797 & 52 \\
\hline 100 & 99.917 & 172 & 99.957 & 90 & 100.157 & 42 & 100.117 & 38 & 100.037 & 44 & 101.277 & 52 \\
\hline 100 & 99.937 & 168 & 99.957 & 90 & 100.217 & 42 & 100.137 & 38 & 100.157 & 44 & 100.077 & 52 \\
\hline 100 & 99.937 & 170 & 99.957 & 90 & 100.177 & 42 & 100.057 & 38 & 101.077 & 44 & 100.077 & 52 \\
\hline 100 & 99.917 & 170 & 99.997 & 90 & 100.177 & 42 & 100.097 & 38 & 100.057 & 44 & 101.717 & 52 \\
\hline 100 & 99.917 & 170 & 99.937 & 90 & 100.237 & 42 & 100.177 & 40 & 100.097 & 44 & 100.297 & 52 \\
\hline 100 & 99.917 & 170 & 99.957 & 90 & 100.197 & 42 & 100.097 & 38 & 100.117 & 44 & 101.517 & 52 \\
\hline 100 & 99.897 & 170 & 99.957 & 90 & 100.237 & 42 & 100.077 & 38 & 100.157 & 44 & 101.497 & 52 \\
\hline 100 & 99.917 & 170 & 99.977 & 90 & 100.237 & 42 & 100.137 & 38 & 100.077 & 44 & 101.417 & 52 \\
\hline 100 & 99.957 & 170 & 99.977 & 90 & 100.177 & 42 & 100.017 & 40 & 100.137 & 46 & 99.937 & 52 \\
\hline
\end{tabular}


Table B.5 Angle of Laser Incidence: $10 \mathrm{~m}$ to $100 \mathrm{~m}$

Attic, Bldg. 226

No Filter

Test Dates: Nov. 1, 5, and 28, 2001

File: rot_calib_11_01_01.xls

\begin{tabular}{|c|c|c|c|c|c|c|c|c|c|c|}
\hline \multirow{3}{*}{$\frac{\text { Distance }}{(\mathrm{m})}$} & \multicolumn{10}{|c|}{$\begin{array}{c}\text { Silver, shiny } \\
\text { Rotation Angle }\left(^{\circ}\right)\end{array}$} \\
\hline & \multicolumn{2}{|c|}{60} & \multicolumn{2}{|c|}{70} & \multicolumn{2}{|c|}{80} & \multicolumn{2}{|c|}{85} & \multicolumn{2}{|c|}{90} \\
\hline & Dist. (m) & Inten. & Dist. (m) & Inten. & Dist. (m) & Inten. & Dist. (m) & Inten. & Dist. (m) & Inten. \\
\hline 10 & 11.109 & 102 & 11.53 & 74 & 13.333 & 72 & 9.887 & 50 & 9.567 & 74 \\
\hline 10 & 11.169 & 102 & 11.53 & 74 & 13.353 & 72 & 9.867 & 52 & 9.567 & 74 \\
\hline 10 & 11.169 & 102 & 11.55 & 74 & 13.333 & 72 & 9.987 & 52 & 9.607 & 74 \\
\hline 10 & 11.129 & 102 & 11.55 & 74 & 13.333 & 74 & 9.847 & 52 & 9.607 & 74 \\
\hline 10 & 11.169 & 102 & 11.57 & 74 & 13.333 & 72 & 9.887 & 52 & 9.562 & 74 \\
\hline 10 & 11.109 & 102 & 11.55 & 74 & 13.373 & 72 & 9.907 & 50 & 9.587 & 74 \\
\hline 10 & 11.149 & 102 & 11.55 & 74 & 13.353 & 72 & 9.887 & 50 & 9.567 & 74 \\
\hline 10 & 11.169 & 102 & 11.53 & 74 & 13.313 & 74 & 9.887 & 52 & 9.607 & 74 \\
\hline 10 & 11.169 & 102 & 11.57 & 74 & 13.333 & 72 & 9.807 & 50 & 9.567 & 74 \\
\hline 10 & 11.129 & 102 & 11.55 & 74 & 13.313 & 72 & 9.867 & 50 & 9.607 & 74 \\
\hline 20 & 19.902 & 66 & 20.042 & 48 & 20.142 & 56 & 19.882 & 60 & 19.782 & 76 \\
\hline 20 & 19.902 & 66 & 20.062 & 48 & 20.182 & 56 & 19.862 & 60 & 19.962 & 76 \\
\hline 20 & 19.882 & 66 & 20.022 & 48 & 20.082 & 58 & 19.822 & 58 & 19.802 & 78 \\
\hline 20 & 19.882 & 66 & 20.042 & 48 & 20.162 & 58 & 19.842 & 58 & 19.822 & 78 \\
\hline 20 & 19.902 & 64 & 20.062 & 48 & 20.142 & 58 & 19.842 & 60 & 19.802 & 78 \\
\hline 20 & 19.902 & 64 & 20.082 & 48 & 20.142 & 56 & 19.882 & 58 & 19.802 & 78 \\
\hline 20 & 19.902 & 66 & 20.002 & 48 & 20.202 & 58 & 19.842 & 60 & 19.802 & 78 \\
\hline 20 & 19.922 & 64 & 20.042 & 48 & 20.082 & 58 & 19.842 & 60 & 19.762 & 78 \\
\hline 20 & 19.882 & 66 & 20.062 & 48 & 20.162 & 56 & 19.862 & 60 & 19.802 & 78 \\
\hline 20 & 19.902 & 66 & 20.062 & 48 & 20.142 & 58 & 19.882 & 60 & 19.782 & 78 \\
\hline 30 & 31.22 & 66 & 31.956 & 56 & 30.276 & 62 & 30.036 & 58 & 29.776 & 68 \\
\hline 30 & 31.24 & 66 & 31.96 & 56 & 30.136 & 62 & 29.976 & 58 & 29.796 & 68 \\
\hline 30 & 31.216 & 66 & 31.976 & 56 & 30.196 & 62 & 29.996 & 58 & 29.776 & 68 \\
\hline 30 & 31.216 & 66 & 31.976 & 56 & 30.056 & 62 & 30.056 & 58 & 29.756 & 68 \\
\hline 30 & 31.196 & 66 & 31.956 & 56 & 30.076 & 62 & 30.016 & 58 & 29.756 & 68 \\
\hline 30 & 31.176 & 66 & 31.956 & 56 & 30.176 & 62 & 29.896 & 58 & 29.836 & 68 \\
\hline 30 & 31.216 & 66 & 31.936 & 56 & 30.176 & 62 & 29.936 & 58 & 29.836 & 68 \\
\hline 30 & 31.196 & 66 & 31.976 & 56 & 30.096 & 60 & 29.956 & 58 & 29.796 & 68 \\
\hline 30 & 31.176 & 66 & 31.936 & 56 & 30.216 & 62 & 29.936 & 58 & 29.816 & 68 \\
\hline 30 & 31.196 & 66 & 31.916 & 56 & 30.156 & 62 & 29.896 & 58 & 29.816 & 68 \\
\hline 40 & 41.576 & 52 & 40.411 & 42 & 39.951 & 50 & 39.871 & 54 & 39.651 & 64 \\
\hline 40 & 41.576 & 52 & 40.596 & 42 & 39.851 & 48 & 39.951 & 54 & 39.711 & 64 \\
\hline 40 & 41.551 & 52 & 40.451 & 42 & 39.871 & 50 & 39.931 & 54 & 39.731 & 64 \\
\hline 40 & 41.571 & 52 & 40.651 & 42 & 39.911 & 50 & 39.951 & 54 & 39.691 & 64 \\
\hline 40 & 41.596 & 52 & 40.431 & 42 & 39.871 & 48 & 39.991 & 54 & 39.691 & 64 \\
\hline 40 & 41.591 & 52 & 40.371 & 42 & 39.971 & 50 & 39.831 & 54 & 39.651 & 66 \\
\hline 40 & 41.611 & 52 & 40.531 & 42 & 39.891 & 48 & 40.051 & 54 & 39.651 & 64 \\
\hline 40 & 41.556 & 52 & 40.491 & 42 & 39.891 & 50 & 39.971 & 54 & 39.691 & 64 \\
\hline 40 & 41.551 & 52 & 40.551 & 42 & 39.891 & 50 & 39.831 & 54 & 39.691 & 64 \\
\hline 40 & 41.551 & 50 & 40.531 & 42 & 39.911 & 48 & 39.811 & 54 & 39.671 & 66 \\
\hline 50 & 51.242 & 48 & 52.542 & 78 & 50.382 & 52 & 50.042 & 54 & 49.602 & 74 \\
\hline 50 & 50.962 & 46 & 51.742 & 54 & 51.022 & 50 & 49.982 & 54 & 109.317 & 74 \\
\hline 50 & 50.982 & 46 & 51.742 & 52 & 50.722 & 50 & 50.002 & 54 & 109.317 & 74 \\
\hline 50 & 50.982 & 46 & 51.722 & 54 & 51.642 & 50 & 50.062 & 54 & 109.337 & 74 \\
\hline 50 & 51.022 & 48 & 51.737 & 54 & 50.982 & 52 & 49.842 & 88 & 109.322 & 74 \\
\hline 50 & 51.022 & 48 & 51.762 & 54 & 50.862 & 52 & 50.157 & 56 & 109.282 & 74 \\
\hline 50 & 50.962 & 46 & 51.722 & 54 & 50.902 & 52 & 50.162 & 54 & 109.322 & 74 \\
\hline 50 & 50.982 & 46 & 51.722 & 54 & 50.822 & 52 & 50.022 & 54 & 109.342 & 74 \\
\hline 50 & 50.982 & 48 & 51.722 & 54 & 50.742 & 52 & 50.077 & 54 & 49.722 & 74 \\
\hline 50 & 50.982 & 48 & 51.722 & 54 & 50.322 & 52 & 50.022 & 54 & 109.297 & 74 \\
\hline 60 & 60.845 & 64 & 60.96 & 48 & 62.6 & 66 & 59.736 & 68 & 59.636 & 74 \\
\hline 60 & 60.86 & 64 & 61 & 48 & 62.56 & 66 & 59.816 & 68 & 59.596 & 74 \\
\hline 60 & 60.86 & 64 & 60.98 & 48 & 62.576 & 66 & 59.796 & 68 & 59.656 & 74 \\
\hline 60 & 60.9 & 64 & 60.92 & 48 & 62.54 & 66 & 59.796 & 68 & 59.616 & 74 \\
\hline 60 & 60.885 & 64 & 61.02 & 48 & 62.52 & 66 & 59.876 & 68 & 59.536 & 74 \\
\hline 60 & 60.88 & 64 & 61.02 & 48 & 62.516 & 66 & 59.916 & 68 & 59.616 & 74 \\
\hline 60 & 60.88 & 64 & 60.92 & 48 & 62.52 & 66 & 59.916 & 68 & 59.536 & 74 \\
\hline
\end{tabular}


Table B.5 Angle of Laser Incidence: $10 \mathrm{~m}$ to $100 \mathrm{~m}$

\begin{tabular}{|c|c|c|c|c|c|c|c|c|c|c|}
\hline \multicolumn{9}{|c|}{$\begin{array}{l}\text { Attic, Bldg. } 226 \\
\text { No Filter } \\
\text { Test Dates: Nov. 1, 5, and 28, } 2001\end{array}$} & \multicolumn{2}{|c|}{ File: rot_calib_11_01_01.xls } \\
\hline \multirow[b]{3}{*}{ Distance } & \multirow{2}{*}{\multicolumn{10}{|c|}{$\begin{array}{c}\text { Silver, shiny } \\
\text { Rotation Angle }\left({ }^{\circ}\right)\end{array}$}} \\
\hline & & & & & & & & & & \\
\hline & \multicolumn{2}{|c|}{60} & \multicolumn{2}{|c|}{70} & \multicolumn{2}{|c|}{80} & \multicolumn{2}{|c|}{85} & \multicolumn{2}{|c|}{90} \\
\hline$(\mathrm{m})$ & Dist. (m) & Inten. & Dist. (m) & Inten. & Dist. (m) & Inten. & Dist. (m) & Inten. & Dist. (m) & Inten. \\
\hline 60 & 60.86 & 64 & 60.96 & 48 & 62.576 & 66 & 59.996 & 68 & 59.636 & 74 \\
\hline 60 & 60.86 & 64 & 61.02 & 48 & 62.556 & 66 & 59.956 & 68 & 59.636 & 74 \\
\hline 60 & 60.84 & 64 & 61 & 48 & 62.476 & 66 & 59.676 & 68 & 59.596 & 74 \\
\hline 70 & 71.122 & 42 & 72.022 & 54 & 73.302 & 62 & 69.837 & 66 & 69.577 & 70 \\
\hline 70 & 71.122 & 44 & 71.882 & 52 & 73.402 & 62 & 90.457 & 66 & 69.717 & 70 \\
\hline 70 & 71.102 & 44 & 71.962 & 54 & 73.342 & 62 & 90.682 & 66 & 69.717 & 70 \\
\hline 70 & 71.162 & 44 & 71.902 & 52 & 73.422 & 62 & 90.697 & 66 & 69.757 & 70 \\
\hline 70 & 71.102 & 44 & 71.862 & 52 & 73.362 & 62 & 90.637 & 66 & 69.557 & 70 \\
\hline 70 & 71.142 & 44 & 71.922 & 52 & 73.277 & 62 & 90.437 & 66 & 69.697 & 70 \\
\hline 70 & 71.122 & 44 & 72.017 & 52 & 73.317 & 62 & 90.602 & 66 & 69.657 & 72 \\
\hline 70 & 71.022 & 44 & 72.042 & 52 & 73.317 & 62 & 90.477 & 66 & 69.697 & 70 \\
\hline 70 & 71.137 & 44 & 71.982 & 52 & 73.337 & 62 & 90.737 & 66 & 69.762 & 70 \\
\hline 70 & 71.122 & 44 & 71.902 & 54 & 73.377 & 62 & 90.577 & 66 & 69.677 & 70 \\
\hline 80 & 79.977 & 40 & 90.717 & 64 & 90.797 & 66 & 90.197 & 68 & 79.637 & 74 \\
\hline 80 & 80.057 & 40 & 90.897 & 64 & 90.877 & 66 & 90.272 & 68 & 79.617 & 72 \\
\hline 80 & 79.937 & 40 & 90.777 & 64 & 91.137 & 66 & 90.257 & 68 & 79.617 & 74 \\
\hline 80 & 79.997 & 40 & 90.597 & 64 & 90.772 & 66 & 90.257 & 70 & 79.492 & 74 \\
\hline 80 & 79.997 & 40 & 90.777 & 64 & 91.017 & 66 & 90.257 & 68 & 79.557 & 74 \\
\hline 80 & 79.997 & 40 & 109.957 & 64 & 90.727 & 66 & 90.312 & 68 & 79.953 & 74 \\
\hline 80 & 79.997 & 40 & 110.112 & 62 & 91.072 & 66 & 90.197 & 68 & 79.537 & 74 \\
\hline 80 & 79.977 & 40 & 90.797 & 64 & 90.957 & 66 & 90.377 & 70 & 79.577 & 74 \\
\hline 80 & 79.992 & 40 & 90.817 & 64 & 90.977 & 68 & 90.377 & 68 & 79.577 & 74 \\
\hline 80 & 79.997 & 40 & 90.557 & 64 & 90.617 & 66 & 90.317 & 68 & 79.697 & 74 \\
\hline 90 & 91.197 & 56 & 109.117 & 64 & 94.397 & 68 & 97.237 & 68 & 89.657 & 72 \\
\hline 90 & 91.917 & 56 & 109.137 & 64 & 94.457 & 68 & 97.117 & 70 & 89.777 & 72 \\
\hline 90 & 91.277 & 56 & 91.737 & 64 & 94.397 & 68 & 96.897 & 70 & 89.777 & 72 \\
\hline 90 & 91.177 & 56 & 109.117 & 64 & 94.397 & 68 & 97.277 & 70 & 89.717 & 72 \\
\hline 90 & 91.237 & 56 & 91.837 & 64 & 94.497 & 68 & 96.997 & 70 & 89.737 & 72 \\
\hline 90 & 91.297 & 56 & 109.137 & 64 & 94.417 & 68 & 97.157 & 70 & 89.637 & 72 \\
\hline 90 & 91.197 & 56 & 109.137 & 64 & 94.457 & 68 & 97.017 & 70 & 89.737 & 72 \\
\hline 90 & 91.217 & 58 & 91.197 & 64 & 94.597 & 68 & 97.057 & 68 & 89.737 & 72 \\
\hline 90 & 91.277 & 56 & 109.137 & 64 & 94.337 & 68 & 97.237 & 70 & 89.697 & 72 \\
\hline 90 & 91.237 & 54 & 91.697 & 64 & 94.477 & 68 & 97.237 & 68 & 89.757 & 72 \\
\hline 100 & 110.077 & 64 & 109.677 & 66 & 109.537 & 70 & 109.417 & 70 & 99.817 & 76 \\
\hline 100 & 110.037 & 64 & 109.657 & 66 & 109.537 & 70 & 109.417 & 70 & 99.597 & 76 \\
\hline 100 & 109.957 & 64 & 109.657 & 66 & 109.537 & 68 & 109.397 & 70 & 99.677 & 76 \\
\hline 100 & 110.017 & 64 & 109.697 & 66 & 109.537 & 70 & 109.377 & 70 & 99.737 & 76 \\
\hline 100 & 110.097 & 64 & 109.657 & 66 & 109.537 & 70 & 109.397 & 70 & 99.677 & 76 \\
\hline 100 & 110.037 & 64 & 109.577 & 66 & 109.477 & 70 & 109.377 & 70 & 99.617 & 76 \\
\hline 100 & 109.977 & 64 & 109.597 & 66 & 109.537 & 70 & 109.377 & 70 & 99.557 & 76 \\
\hline 100 & 102.777 & 64 & 109.657 & 66 & 109.557 & 70 & 109.397 & 70 & 99.697 & 76 \\
\hline 100 & 109.877 & 64 & 109.717 & 66 & 109.497 & 70 & 109.417 & 70 & 99.757 & 76 \\
\hline 100 & 109.997 & 64 & 109.657 & 66 & 109.517 & 70 & 109.397 & 70 & 99.677 & 76 \\
\hline
\end{tabular}


Table B.5 Angle of Laser Incidence: $10 \mathrm{~m}$ to $100 \mathrm{~m}$

Attic, Bldg. 226

No Filter

Test Dates: Nov. 1, 5, and 28, 2001

File: rot_calib_11_01_01.xls

\begin{tabular}{|c|c|c|c|c|c|c|c|c|c|c|c|c|}
\hline \multirow{4}{*}{$\begin{array}{c}\text { Distance } \\
\text { (m) }\end{array}$} & \multicolumn{12}{|c|}{ White } \\
\hline & \multicolumn{12}{|c|}{ Rotation Angle $\left({ }^{\circ}\right)$} \\
\hline & \multicolumn{2}{|c|}{0} & \multicolumn{2}{|c|}{10} & \multicolumn{2}{|c|}{20} & \multicolumn{2}{|c|}{30} & \multicolumn{2}{|c|}{40} & \multicolumn{2}{|c|}{50} \\
\hline & Dist. (m) & Inten. & Dist. (m) & Inten. & Dist. (m) & Inten. & Dist. (m) & Inten. & Dist. (m) & Inten. & Dist. (m) & Inten. \\
\hline 10 & 9.967 & 150 & 10.003 & 150 & 9.942 & 148 & 10.003 & 148 & 10.003 & 144 & 10.023 & 142 \\
\hline 10 & 9.982 & 150 & 10.008 & 150 & 9.982 & 150 & 10.003 & 148 & 9.982 & 144 & 10.003 & 142 \\
\hline 10 & 9.962 & 150 & 9.982 & 150 & 10.003 & 148 & 9.982 & 148 & 9.982 & 144 & 10.023 & 142 \\
\hline 10 & 10.003 & 150 & 9.962 & 150 & 9.982 & 150 & 9.982 & 146 & 9.982 & 144 & 10.003 & 142 \\
\hline 10 & 9.982 & 150 & 10.003 & 150 & 9.962 & 148 & 10.023 & 148 & 10.003 & 144 & 10.023 & 142 \\
\hline 10 & 9.962 & 150 & 9.982 & 150 & 10.003 & 148 & 10.003 & 148 & 10.003 & 144 & 9.982 & 142 \\
\hline 10 & 9.962 & 150 & 9.982 & 150 & 9.982 & 148 & 9.962 & 146 & 10.003 & 144 & 9.982 & 142 \\
\hline 10 & 9.982 & 150 & 9.942 & 150 & 9.982 & 150 & 10.003 & 148 & 9.962 & 144 & 10.023 & 142 \\
\hline 10 & 10.003 & 150 & 10.003 & 150 & 10.003 & 148 & 10.003 & 148 & 10.003 & 144 & 9.982 & 142 \\
\hline 10 & 9.967 & 150 & 9.942 & 150 & 9.962 & 148 & 9.962 & 146 & 10.003 & 144 & 9.982 & 142 \\
\hline 20 & 19.982 & 150 & 20.002 & 150 & 19.962 & 148 & 20.002 & 146 & 20.002 & 144 & 19.962 & 140 \\
\hline 20 & 19.982 & 150 & 19.962 & 150 & 19.982 & 148 & 19.982 & 146 & 19.962 & 144 & 19.962 & 140 \\
\hline 20 & 20.002 & 150 & 19.982 & 150 & 20.002 & 148 & 20.002 & 146 & 20.002 & 144 & 20.002 & 140 \\
\hline 20 & 19.962 & 150 & 20.002 & 150 & 20.002 & 148 & 20.022 & 146 & 19.962 & 144 & 20.002 & 140 \\
\hline 20 & 19.982 & 150 & 20.002 & 150 & 20.002 & 148 & 20.022 & 146 & 19.982 & 144 & 19.962 & 140 \\
\hline 20 & 20.002 & 150 & 19.962 & 150 & 20.002 & 148 & 20.002 & 146 & 19.982 & 144 & 19.982 & 140 \\
\hline 20 & 20.002 & 150 & 20.002 & 150 & 20.002 & 148 & 20.002 & 146 & 19.982 & 144 & 20.002 & 140 \\
\hline 20 & 19.982 & 150 & 20.002 & 150 & 20.002 & 148 & 20.002 & 146 & 20.002 & 144 & 19.982 & 140 \\
\hline 20 & 20.002 & 150 & 20.002 & 150 & 19.982 & 148 & 20.002 & 146 & 20.002 & 144 & 20.022 & 140 \\
\hline 20 & 19.962 & 150 & 20.002 & 150 & 19.962 & 148 & 20.002 & 146 & 19.962 & 144 & 20.002 & 140 \\
\hline 30 & 29.996 & 138 & 30.016 & 138 & 29.976 & 136 & 29.976 & 134 & 29.971 & 130 & 29.991 & 126 \\
\hline 30 & 29.976 & 138 & 29.976 & 136 & 29.996 & 136 & 30.016 & 134 & 29.951 & 130 & 29.991 & 126 \\
\hline 30 & 29.996 & 138 & 29.976 & 136 & 29.956 & 136 & 29.976 & 134 & 29.991 & 130 & 29.971 & 126 \\
\hline 30 & 30.016 & 138 & 29.976 & 136 & 29.976 & 136 & 29.996 & 134 & 29.991 & 130 & 30.011 & 126 \\
\hline 30 & 30.016 & 138 & 29.996 & 136 & 29.996 & 136 & 29.991 & 134 & 29.951 & 130 & 29.951 & 126 \\
\hline 30 & 29.976 & 138 & 29.996 & 138 & 29.996 & 136 & 29.976 & 134 & 29.991 & 130 & 29.971 & 126 \\
\hline 30 & 29.996 & 138 & 29.936 & 136 & 29.996 & 136 & 29.976 & 134 & 29.971 & 130 & 29.991 & 126 \\
\hline 30 & 29.996 & 138 & 29.976 & 136 & 29.996 & 136 & 30.011 & 134 & 29.971 & 130 & 29.971 & 126 \\
\hline 30 & 30.016 & 138 & 29.996 & 136 & 29.956 & 136 & 29.991 & 134 & 29.991 & 130 & 29.971 & 126 \\
\hline 30 & 29.976 & 138 & 29.976 & 136 & 29.956 & 136 & 29.951 & 134 & 29.971 & 130 & 29.991 & 126 \\
\hline 40 & 39.971 & 120 & 40.011 & 120 & 39.991 & 120 & 39.986 & 118 & 39.966 & 114 & 39.966 & 112 \\
\hline 40 & 39.991 & 122 & 39.951 & 120 & 40.011 & 120 & 39.971 & 118 & 40.011 & 116 & 40.006 & 112 \\
\hline 40 & 39.991 & 122 & 39.991 & 120 & 40.006 & 120 & 39.971 & 118 & 40.006 & 116 & 39.986 & 112 \\
\hline 40 & 39.991 & 122 & 39.951 & 120 & 39.991 & 120 & 40.031 & 118 & 39.986 & 116 & 39.966 & 112 \\
\hline 40 & 40.011 & 122 & 39.971 & 120 & 39.991 & 120 & 39.986 & 118 & 39.986 & 116 & 39.986 & 112 \\
\hline 40 & 40.031 & 122 & 39.951 & 120 & 39.991 & 120 & 39.986 & 118 & 40.006 & 116 & 39.986 & 112 \\
\hline 40 & 39.971 & 122 & 40.011 & 120 & 39.971 & 120 & 39.986 & 118 & 39.986 & 116 & 40.006 & 112 \\
\hline 40 & 39.991 & 122 & 39.951 & 120 & 40.011 & 120 & 39.951 & 118 & 40.006 & 116 & 40.006 & 112 \\
\hline 40 & 40.011 & 122 & 39.971 & 120 & 39.991 & 120 & 39.971 & 118 & 40.006 & 116 & 40.006 & 112 \\
\hline 40 & 39.991 & 122 & 39.991 & 120 & 39.946 & 120 & 39.966 & 118 & 40.006 & 116 & 39.966 & 112 \\
\hline 50 & 49.977 & 108 & 50.017 & 108 & 49.977 & 108 & 50.037 & 106 & 49.997 & 102 & 50.017 & 98 \\
\hline 50 & 49.977 & 108 & 50.017 & 108 & 50.017 & 108 & 50.017 & 106 & 49.977 & 102 & 49.997 & 100 \\
\hline 50 & 49.977 & 108 & 49.997 & 108 & 50.017 & 108 & 49.997 & 106 & 49.997 & 102 & 50.037 & 100 \\
\hline 50 & 49.957 & 108 & 50.017 & 108 & 50.017 & 108 & 50.017 & 106 & 49.997 & 102 & 50.017 & 100 \\
\hline 50 & 49.997 & 108 & 49.977 & 108 & 50.017 & 108 & 50.017 & 106 & 50.037 & 104 & 50.017 & 98 \\
\hline 50 & 49.977 & 108 & 50.017 & 108 & 49.997 & 108 & 50.017 & 106 & 50.017 & 102 & 49.997 & 98 \\
\hline 50 & 49.997 & 108 & 49.982 & 108 & 49.997 & 108 & 50.017 & 106 & 50.022 & 104 & 50.017 & 100 \\
\hline 50 & 49.997 & 108 & 49.997 & 108 & 49.977 & 108 & 50.017 & 106 & 49.977 & 104 & 50.037 & 100 \\
\hline 50 & 49.997 & 108 & 50.017 & 108 & 50.037 & 108 & 49.997 & 106 & 49.997 & 102 & 50.037 & 100 \\
\hline 50 & 49.977 & 108 & 50.017 & 108 & 50.017 & 108 & 49.977 & 106 & 50.017 & 104 & 49.997 & 100 \\
\hline 60 & 59.976 & 100 & 59.991 & 100 & 60.011 & 98 & 59.991 & 96 & 59.971 & 94 & 59.991 & 90 \\
\hline 60 & 59.996 & 100 & 59.991 & 100 & 60.011 & 98 & 59.991 & 98 & 59.931 & 94 & 59.971 & 90 \\
\hline 60 & 59.976 & 100 & 59.991 & 100 & 59.951 & 98 & 59.991 & 96 & 59.991 & 94 & 59.951 & 90 \\
\hline 60 & 60.011 & 100 & 59.971 & 100 & 60.011 & 98 & 59.951 & 96 & 59.971 & 94 & 59.991 & 90 \\
\hline 60 & 60.011 & 100 & 59.951 & 100 & 59.971 & 98 & 59.971 & 98 & 59.971 & 94 & 59.991 & 90 \\
\hline 60 & 59.991 & 100 & 59.991 & 100 & 59.991 & 98 & 59.991 & 96 & 59.991 & 94 & 60.011 & 90 \\
\hline 60 & 59.971 & 100 & 59.931 & 100 & 60.011 & 98 & 59.971 & 96 & 59.971 & 94 & 59.991 & 90 \\
\hline
\end{tabular}


Table B.5 Angle of Laser Incidence: $10 \mathrm{~m}$ to $100 \mathrm{~m}$

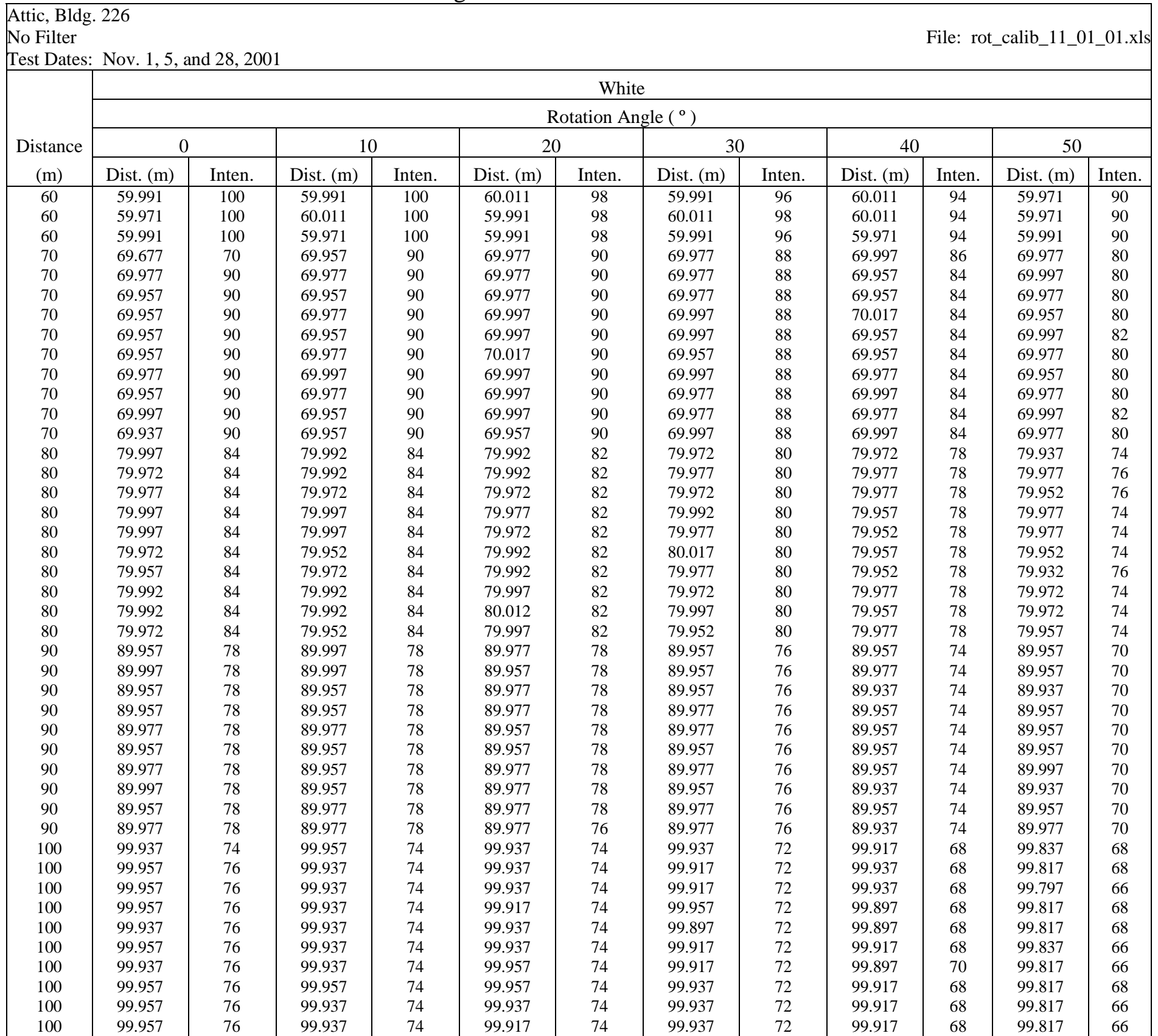


Table B.5 Angle of Laser Incidence: $10 \mathrm{~m}$ to $100 \mathrm{~m}$

\begin{tabular}{|c|c|c|c|c|c|c|c|c|c|c|}
\hline \multicolumn{11}{|c|}{$\begin{array}{l}\text { Attic, Bldg. } 226 \\
\text { No Filter } \\
\text { Test Dates: Nov. 1, 5, and 28, } 2001\end{array}$} \\
\hline \multirow{4}{*}{$\begin{array}{c}\text { Distance } \\
(\mathrm{m})\end{array}$} & \multicolumn{10}{|c|}{ White } \\
\hline & \multicolumn{10}{|c|}{ Rotation Angle $\left({ }^{\circ}\right)$} \\
\hline & \multicolumn{2}{|c|}{60} & \multicolumn{2}{|c|}{70} & \multicolumn{2}{|c|}{80} & \multicolumn{2}{|c|}{85} & \multicolumn{2}{|c|}{90} \\
\hline & Dist. (m) & Inten. & Dist. (m) & Inten. & Dist. (m) & Inten. & Dist. (m) & Inten. & Dist. (m) & Inten. \\
\hline 10 & 9.962 & 136 & 9.962 & 132 & 9.982 & 126 & 10.023 & 118 & 9.762 & 74 \\
\hline 10 & 10.003 & 136 & 10.003 & 132 & 9.982 & 126 & 10.003 & 118 & 9.722 & 74 \\
\hline 10 & 9.962 & 136 & 10.003 & 132 & 10.003 & 126 & 10.023 & 118 & 9.762 & 74 \\
\hline 10 & 10.003 & 136 & 10.003 & 132 & 10.003 & 126 & 10.003 & 118 & 9.762 & 74 \\
\hline 10 & 10.003 & 136 & 9.962 & 132 & 10.023 & 126 & 10.023 & 118 & 9.682 & 74 \\
\hline 10 & 9.982 & 136 & 10.003 & 132 & 9.962 & 126 & 10.023 & 118 & 9.762 & 74 \\
\hline 10 & 10.023 & 136 & 10.023 & 132 & 10.003 & 128 & 10.023 & 118 & 9.742 & 74 \\
\hline 10 & 9.962 & 136 & 10.003 & 132 & 10.023 & 128 & 10.023 & 118 & 9.742 & 74 \\
\hline 10 & 9.982 & 136 & 10.003 & 132 & 10.003 & 126 & 10.023 & 118 & 9.742 & 74 \\
\hline 10 & 10.023 & 138 & 9.962 & 132 & 10.003 & 128 & 9.982 & 118 & 9.762 & 74 \\
\hline 20 & 19.982 & 136 & 20.002 & 132 & 20.042 & 124 & 20.022 & 112 & 19.802 & 104 \\
\hline 20 & 20.002 & 136 & 20.022 & 132 & 20.022 & 124 & 20.002 & 112 & 19.842 & 104 \\
\hline 20 & 20.002 & 136 & 19.982 & 132 & 20.022 & 124 & 20.022 & 112 & 19.802 & 104 \\
\hline 20 & 20.022 & 136 & 20.022 & 132 & 20.042 & 124 & 20.002 & 112 & 19.822 & 104 \\
\hline 20 & 20.002 & 136 & 20.022 & 132 & 20.042 & 124 & 19.982 & 112 & 19.842 & 104 \\
\hline 20 & 20.002 & 136 & 19.982 & 132 & 20.022 & 124 & 19.982 & 112 & 19.842 & 104 \\
\hline 20 & 20.022 & 136 & 19.982 & 132 & 20.002 & 124 & 20.002 & 112 & 19.822 & 104 \\
\hline 20 & 20.002 & 136 & 19.982 & 132 & 20.022 & 124 & 19.982 & 112 & 19.822 & 102 \\
\hline 20 & 20.002 & 136 & 20.042 & 132 & 20.042 & 124 & 20.002 & 112 & 19.802 & 104 \\
\hline 20 & 19.962 & 136 & 20.022 & 132 & 20.042 & 124 & 19.962 & 112 & 19.842 & 104 \\
\hline 30 & 29.971 & 122 & 29.991 & 116 & 29.971 & 100 & 30.071 & 82 & 29.751 & 70 \\
\hline 30 & 30.011 & 122 & 29.991 & 116 & 29.971 & 100 & 30.011 & 82 & 29.771 & 70 \\
\hline 30 & 29.991 & 122 & 29.991 & 116 & 29.911 & 100 & 30.071 & 82 & 29.751 & 72 \\
\hline 30 & 29.991 & 122 & 30.011 & 116 & 29.951 & 100 & 30.031 & 82 & 29.731 & 70 \\
\hline 30 & 30.011 & 122 & 29.991 & 116 & 29.911 & 100 & 30.051 & 82 & 29.751 & 70 \\
\hline 30 & 29.991 & 122 & 30.011 & 116 & 29.931 & 100 & 30.051 & 82 & 29.771 & 70 \\
\hline 30 & 29.991 & 122 & 29.991 & 116 & 29.951 & 100 & 30.051 & 82 & 29.771 & 70 \\
\hline 30 & 29.991 & 122 & 29.991 & 116 & 29.951 & 100 & 30.051 & 82 & 29.771 & 72 \\
\hline 30 & 30.011 & 122 & 30.011 & 116 & 29.931 & 100 & 30.051 & 82 & 29.811 & 70 \\
\hline 30 & 29.991 & 122 & 30.011 & 116 & 29.931 & 100 & 30.051 & 82 & 29.751 & 70 \\
\hline 40 & 40.006 & 108 & 39.966 & 100 & 39.966 & 78 & 39.986 & 74 & 39.846 & 70 \\
\hline 40 & 40.026 & 108 & 40.006 & 100 & 39.966 & 80 & 39.966 & 74 & 39.846 & 70 \\
\hline 40 & 40.006 & 108 & 39.966 & 100 & 39.946 & 80 & 39.966 & 72 & 39.826 & 70 \\
\hline 40 & 40.006 & 108 & 39.986 & 100 & 39.946 & 78 & 39.966 & 74 & 39.846 & 70 \\
\hline 40 & 40.026 & 108 & 40.006 & 100 & 39.966 & 80 & 39.966 & 74 & 39.846 & 70 \\
\hline 40 & 40.006 & 108 & 40.006 & 100 & 39.966 & 80 & 39.946 & 74 & 39.826 & 70 \\
\hline 40 & 40.006 & 108 & 40.006 & 100 & 39.966 & 78 & 39.966 & 74 & 39.846 & 70 \\
\hline 40 & 40.026 & 108 & 39.986 & 100 & 39.966 & 78 & 39.966 & 72 & 39.806 & 70 \\
\hline 40 & 39.966 & 108 & 40.006 & 100 & 39.966 & 78 & 39.966 & 72 & 39.866 & 70 \\
\hline 40 & 39.986 & 108 & 39.986 & 100 & 39.966 & 78 & 39.966 & 74 & 39.846 & 70 \\
\hline 50 & 50.037 & 94 & 49.977 & 82 & 49.977 & 72 & 50.037 & 58 & 49.697 & 74 \\
\hline 50 & 49.997 & 94 & 49.957 & 82 & 49.957 & 72 & 50.037 & 58 & 49.717 & 74 \\
\hline 50 & 49.977 & 94 & 49.977 & 82 & 49.957 & 72 & 50.057 & 58 & 49.897 & 74 \\
\hline 50 & 49.977 & 94 & 49.977 & 82 & 49.937 & 72 & 50.037 & 58 & 49.617 & 74 \\
\hline 50 & 49.997 & 94 & 49.977 & 82 & 49.957 & 72 & 50.077 & 58 & 49.797 & 74 \\
\hline 50 & 50.017 & 94 & 49.977 & 82 & 49.957 & 72 & 50.077 & 58 & 49.657 & 74 \\
\hline 50 & 49.977 & 94 & 49.997 & 82 & 49.917 & 72 & 50.017 & 58 & 49.777 & 74 \\
\hline 50 & 49.997 & 94 & 49.937 & 82 & 49.917 & 72 & 50.037 & 58 & 49.837 & 74 \\
\hline 50 & 49.997 & 94 & 49.957 & 82 & 49.957 & 72 & 50.037 & 58 & 49.797 & 74 \\
\hline 50 & 50.037 & 94 & 49.957 & 82 & 49.937 & 72 & 50.037 & 58 & 49.797 & 74 \\
\hline 60 & 59.991 & 84 & 59.986 & 70 & 59.926 & 62 & 59.866 & 62 & 59.646 & 70 \\
\hline 60 & 59.991 & 84 & 59.966 & 70 & 59.946 & 60 & 59.886 & 62 & 59.726 & 70 \\
\hline 60 & 59.971 & 84 & 59.946 & 70 & 59.966 & 62 & 59.926 & 62 & 59.726 & 70 \\
\hline 60 & 60.006 & 84 & 59.946 & 70 & 59.946 & 62 & 59.906 & 62 & 59.686 & 70 \\
\hline 60 & 59.966 & 84 & 59.906 & 70 & 59.946 & 62 & 59.886 & 62 & 59.726 & 70 \\
\hline 60 & 60.011 & 84 & 59.966 & 70 & 59.926 & 62 & 59.886 & 64 & 59.726 & 70 \\
\hline 60 & 60.006 & 84 & 59.926 & 70 & 59.946 & 62 & 59.886 & 62 & 59.746 & 70 \\
\hline
\end{tabular}




\begin{tabular}{|c|c|c|c|c|c|c|c|c|c|c|}
\hline 60 & 59.966 & 84 & 59.946 & 70 & 59.986 & 62 & 59.906 & 62 & 59.706 & 70 \\
\hline 60 & 59.966 & 84 & 59.926 & 70 & 59.946 & 62 & 59.926 & 62 & 59.766 & 70 \\
\hline 60 & 59.971 & 84 & 59.946 & 70 & 59.926 & 62 & 59.926 & 62 & 59.686 & 70 \\
\hline 70 & 69.957 & 76 & 69.957 & 60 & 69.957 & 58 & 69.937 & 60 & 69.697 & 70 \\
\hline 70 & 69.957 & 76 & 69.937 & 60 & 69.977 & 58 & 69.957 & 60 & 69.697 & 70 \\
\hline 70 & 69.957 & 76 & 69.977 & 60 & 69.977 & 58 & 69.977 & 60 & 69.657 & 70 \\
\hline 70 & 69.957 & 76 & 69.957 & 60 & 69.937 & 58 & 69.957 & 60 & 69.717 & 70 \\
\hline 70 & 69.977 & 76 & 69.997 & 60 & 69.997 & 58 & 70.017 & 60 & 69.717 & 70 \\
\hline 70 & 69.957 & 76 & 69.997 & 60 & 69.977 & 58 & 69.957 & 60 & 69.637 & 70 \\
\hline 70 & 69.977 & 76 & 69.997 & 60 & 69.997 & 58 & 69.937 & 60 & 69.677 & 70 \\
\hline 70 & 69.977 & 76 & 69.957 & 60 & 69.997 & 58 & 69.997 & 60 & 69.717 & 70 \\
\hline 70 & 69.977 & 76 & 69.977 & 60 & 70.017 & 58 & 69.957 & 60 & 69.637 & 70 \\
\hline 70 & 69.977 & 76 & 69.957 & 60 & 69.997 & 58 & 69.957 & 60 & 69.797 & 70 \\
\hline 80 & 79.957 & 66 & 79.932 & 60 & 79.872 & 62 & 79.812 & 66 & 79.577 & 72 \\
\hline 80 & 79.952 & 66 & 79.957 & 58 & 79.832 & 64 & 79.852 & 66 & 79.617 & 72 \\
\hline 80 & 79.932 & 66 & 79.932 & 60 & 79.892 & 62 & 79.857 & 66 & 79.592 & 72 \\
\hline 80 & 79.957 & 66 & 79.957 & 60 & 79.897 & 62 & 79.837 & 66 & 79.617 & 72 \\
\hline 80 & 79.952 & 66 & 79.932 & 60 & 79.857 & 62 & 79.832 & 66 & 79.677 & 72 \\
\hline 80 & 79.977 & 66 & 79.952 & 58 & 79.872 & 62 & 79.827 & 66 & 79.592 & 72 \\
\hline 80 & 79.932 & 66 & 79.952 & 58 & 79.852 & 62 & 79.852 & 66 & 79.517 & 72 \\
\hline 80 & 79.897 & 66 & 79.957 & 60 & 79.832 & 62 & 79.817 & 66 & 79.692 & 72 \\
\hline 80 & 79.937 & 66 & 79.912 & 60 & 79.892 & 62 & 79.812 & 66 & 79.692 & 72 \\
\hline 80 & 79.937 & 66 & 79.912 & 58 & 79.892 & 62 & 79.832 & 66 & 79.592 & 72 \\
\hline 90 & 89.977 & 58 & 89.817 & 64 & 89.837 & 66 & 89.817 & 68 & 89.717 & 72 \\
\hline 90 & 89.937 & 58 & 89.837 & 66 & 89.857 & 66 & 89.837 & 70 & 89.677 & 72 \\
\hline 90 & 89.937 & 58 & 89.817 & 66 & 89.837 & 68 & 89.877 & 68 & 89.737 & 72 \\
\hline 90 & 89.977 & 58 & 89.837 & 66 & 89.817 & 66 & 89.877 & 68 & 89.697 & 74 \\
\hline 90 & 89.957 & 58 & 89.857 & 64 & 89.837 & 66 & 89.857 & 68 & 89.697 & 72 \\
\hline 90 & 89.977 & 58 & 89.817 & 66 & 89.837 & 66 & 89.857 & 68 & 89.597 & 74 \\
\hline 90 & 89.937 & 58 & 89.817 & 66 & 89.877 & 68 & 89.817 & 68 & 89.677 & 72 \\
\hline 90 & 89.937 & 58 & 89.797 & 66 & 89.837 & 68 & 89.837 & 68 & 89.617 & 72 \\
\hline 90 & 89.937 & 58 & 89.857 & 64 & 89.817 & 68 & 89.837 & 68 & 89.657 & 72 \\
\hline 90 & 89.957 & 58 & 89.857 & 64 & 89.817 & 68 & 89.817 & 68 & 89.657 & 74 \\
\hline 100 & 99.717 & 72 & 99.717 & 74 & 99.737 & 74 & 99.637 & 76 & 99.817 & 74 \\
\hline 100 & 99.717 & 72 & 99.737 & 74 & 99.737 & 74 & 99.657 & 76 & 99.737 & 74 \\
\hline 100 & 99.717 & 72 & 99.677 & 74 & 99.757 & 76 & 99.757 & 76 & 99.997 & 74 \\
\hline 100 & 99.717 & 72 & 99.717 & 74 & 99.817 & 74 & 99.717 & 76 & 109.157 & 74 \\
\hline 100 & 99.717 & 72 & 99.737 & 74 & 99.717 & 76 & 99.717 & 76 & 99.757 & 74 \\
\hline 100 & 99.737 & 72 & 99.737 & 74 & 99.757 & 74 & 99.657 & 76 & 99.857 & 74 \\
\hline 100 & 99.677 & 72 & 99.737 & 74 & 99.817 & 76 & 99.637 & 76 & 99.857 & 74 \\
\hline 100 & 99.737 & 72 & 99.717 & 74 & 99.717 & 74 & 99.697 & 76 & 99.577 & 74 \\
\hline 100 & 99.697 & 72 & 99.677 & 74 & 99.717 & 76 & 99.717 & 76 & 109.177 & 74 \\
\hline 100 & 99.717 & 72 & 99.717 & 74 & 99.717 & 74 & 99.677 & 76 & 99.777 & 74 \\
\hline
\end{tabular}


Table B.5 Angle of Laser Incidence: $10 \mathrm{~m}$ to $100 \mathrm{~m}$

Attic, Bldg. 226

No Filter

Test Dates: Nov. 1, 5, and 28, 2001

File: rot_calib_11_01_01.xls

\begin{tabular}{|c|c|c|c|c|c|c|c|c|c|c|c|c|}
\hline \multirow{4}{*}{$\begin{array}{c}\text { Distance } \\
(\mathrm{m})\end{array}$} & \multicolumn{12}{|c|}{ Black } \\
\hline & \multicolumn{12}{|c|}{ Rotation Angle $\left({ }^{\circ}\right)$} \\
\hline & \multicolumn{2}{|c|}{0} & \multicolumn{2}{|c|}{10} & \multicolumn{2}{|c|}{20} & \multicolumn{2}{|c|}{30} & \multicolumn{2}{|c|}{40} & \multicolumn{2}{|c|}{50} \\
\hline & Dist. (m) & Inten. & Dist. (m) & Inten. & Dist. (m) & Inten. & Dist. (m) & Inten. & Dist. (m) & Inten. & Dist. (m) & Inten. \\
\hline 10 & 10.023 & 76 & 9.962 & 74 & 10.003 & 68 & 10.023 & 60 & 10.003 & 48 & 10.023 & 42 \\
\hline 10 & 10.003 & 76 & 10.003 & 74 & 10.003 & 68 & 10.023 & 58 & 10.003 & 48 & 10.003 & 42 \\
\hline 10 & 9.962 & 76 & 10.003 & 74 & 9.982 & 68 & 10.003 & 58 & 10.043 & 48 & 10.043 & 42 \\
\hline 10 & 10.023 & 78 & 10.003 & 74 & 9.982 & 68 & 9.982 & 60 & 10.063 & 48 & 10.023 & 42 \\
\hline 10 & 10.003 & 76 & 10.003 & 74 & 9.982 & 68 & 9.982 & 60 & 10.043 & 48 & 10.063 & 42 \\
\hline 10 & 9.962 & 76 & 9.982 & 74 & 10.003 & 68 & 10.003 & 60 & 10.063 & 48 & 10.023 & 42 \\
\hline 10 & 10.003 & 78 & 9.962 & 74 & 10.003 & 68 & 10.003 & 60 & 10.023 & 48 & 9.942 & 42 \\
\hline 10 & 10.023 & 78 & 9.962 & 74 & 9.982 & 68 & 10.023 & 60 & 10.023 & 48 & 10.043 & 42 \\
\hline 10 & 10.003 & 78 & 10.003 & 74 & 10.003 & 68 & 10.023 & 60 & 10.003 & 48 & 10.003 & 42 \\
\hline 10 & 10.003 & 76 & 9.962 & 74 & 10.003 & 68 & 9.962 & 60 & 10.023 & 48 & 9.982 & 42 \\
\hline 20 & 19.982 & 78 & 20.022 & 76 & 19.982 & 70 & 20.002 & 62 & 20.062 & 52 & 20.042 & 44 \\
\hline 20 & 19.982 & 78 & 20.002 & 76 & 19.962 & 70 & 20.002 & 62 & 20.002 & 52 & 20.042 & 46 \\
\hline 20 & 19.982 & 78 & 20.002 & 76 & 19.962 & 70 & 19.982 & 62 & 20.022 & 50 & 20.002 & 44 \\
\hline 20 & 19.982 & 78 & 19.982 & 76 & 20.022 & 70 & 20.002 & 62 & 20.002 & 50 & 19.982 & 44 \\
\hline 20 & 20.002 & 78 & 20.002 & 76 & 20.002 & 70 & 20.002 & 60 & 20.002 & 50 & 20.042 & 44 \\
\hline 20 & 19.982 & 78 & 19.982 & 76 & 19.982 & 70 & 19.982 & 60 & 19.982 & 52 & 20.002 & 44 \\
\hline 20 & 20.002 & 78 & 19.962 & 76 & 19.982 & 70 & 20.002 & 60 & 20.042 & 52 & 19.982 & 44 \\
\hline 20 & 19.982 & 78 & 20.002 & 76 & 20.002 & 70 & 20.022 & 62 & 20.002 & 52 & 20.022 & 44 \\
\hline 20 & 20.002 & 78 & 19.962 & 76 & 19.962 & 70 & 20.002 & 60 & 19.962 & 50 & 19.982 & 44 \\
\hline 20 & 20.002 & 78 & 20.002 & 76 & 20.002 & 70 & 20.022 & 60 & 20.022 & 52 & 20.022 & 44 \\
\hline 30 & 29.991 & 68 & 29.971 & 66 & 30.031 & 58 & 30.011 & 48 & 30.011 & 40 & 30.031 & 36 \\
\hline 30 & 29.971 & 68 & 29.991 & 66 & 29.971 & 58 & 29.971 & 48 & 30.051 & 42 & 29.991 & 36 \\
\hline 30 & 29.991 & 68 & 29.971 & 66 & 29.991 & 58 & 30.011 & 48 & 30.031 & 42 & 30.011 & 36 \\
\hline 30 & 29.951 & 68 & 29.951 & 66 & 29.991 & 58 & 29.971 & 48 & 29.971 & 40 & 29.971 & 36 \\
\hline 30 & 29.991 & 68 & 29.951 & 66 & 30.011 & 58 & 29.991 & 48 & 29.946 & 40 & 30.031 & 36 \\
\hline 30 & 29.991 & 68 & 29.931 & 66 & 29.991 & 58 & 30.011 & 48 & 29.991 & 42 & 29.986 & 36 \\
\hline 30 & 29.971 & 68 & 29.951 & 66 & 29.991 & 58 & 30.011 & 48 & 29.991 & 40 & 29.991 & 36 \\
\hline 30 & 29.971 & 68 & 29.971 & 66 & 29.971 & 58 & 30.011 & 48 & 30.011 & 40 & 30.031 & 36 \\
\hline 30 & 29.971 & 68 & 29.931 & 66 & 29.971 & 58 & 30.031 & 48 & 30.011 & 40 & 29.986 & 36 \\
\hline 30 & 29.991 & 68 & 29.971 & 66 & 29.991 & 58 & 29.991 & 48 & 30.031 & 40 & 30.046 & 36 \\
\hline 40 & 40.026 & 58 & 39.966 & 56 & 39.986 & 50 & 39.966 & 42 & 39.966 & 34 & 40.066 & 30 \\
\hline 40 & 40.026 & 58 & 39.966 & 56 & 40.026 & 50 & 40.006 & 42 & 40.026 & 34 & 39.946 & 28 \\
\hline 40 & 39.986 & 58 & 40.026 & 56 & 39.986 & 50 & 39.966 & 42 & 39.966 & 34 & 39.946 & 30 \\
\hline 40 & 40.026 & 58 & 39.986 & 56 & 40.026 & 50 & 40.006 & 42 & 40.006 & 34 & 39.966 & 30 \\
\hline 40 & 39.966 & 58 & 39.986 & 56 & 40.066 & 50 & 40.006 & 42 & 40.026 & 34 & 40.006 & 30 \\
\hline 40 & 39.946 & 58 & 39.986 & 56 & 40.006 & 50 & 40.006 & 42 & 40.046 & 34 & 39.926 & 30 \\
\hline 40 & 39.966 & 58 & 40.006 & 56 & 40.026 & 50 & 39.986 & 42 & 40.006 & 34 & 40.006 & 30 \\
\hline 40 & 40.006 & 58 & 40.006 & 56 & 40.006 & 50 & 40.006 & 42 & 40.046 & 34 & 39.986 & 30 \\
\hline 40 & 39.986 & 58 & 39.966 & 56 & 39.986 & 50 & 39.986 & 42 & 40.006 & 34 & 40.046 & 30 \\
\hline 40 & 39.986 & 58 & 40.006 & 56 & 39.966 & 50 & 40.006 & 42 & 39.986 & 34 & 40.006 & 30 \\
\hline 50 & 49.997 & 50 & 49.977 & 48 & 49.997 & 44 & 49.997 & 36 & 49.977 & 30 & 49.997 & 24 \\
\hline 50 & 49.997 & 48 & 49.997 & 48 & 50.017 & 42 & 49.977 & 36 & 49.997 & 28 & 49.957 & 24 \\
\hline 50 & 49.997 & 48 & 50.017 & 48 & 49.997 & 44 & 50.017 & 36 & 50.037 & 28 & 49.997 & 22 \\
\hline 50 & 50.017 & 50 & 49.977 & 48 & 50.017 & 42 & 50.057 & 36 & 49.977 & 28 & 49.977 & 24 \\
\hline 50 & 49.997 & 50 & 49.977 & 48 & 50.017 & 44 & 50.017 & 36 & 49.977 & 30 & 49.937 & 22 \\
\hline 50 & 49.977 & 50 & 50.017 & 48 & 49.997 & 44 & 50.077 & 36 & 50.017 & 30 & 50.077 & 24 \\
\hline 50 & 49.997 & 48 & 49.957 & 48 & 50.017 & 44 & 49.997 & 36 & 49.997 & 28 & 49.997 & 24 \\
\hline 50 & 49.937 & 48 & 50.037 & 48 & 50.017 & 44 & 50.037 & 36 & 50.077 & 30 & 49.937 & 22 \\
\hline 50 & 49.977 & 50 & 49.997 & 48 & 49.997 & 44 & 50.017 & 36 & 50.077 & 30 & 50.117 & 24 \\
\hline 50 & 49.977 & 50 & 50.057 & 48 & 50.017 & 44 & 50.017 & 36 & 50.017 & 28 & 49.997 & 24 \\
\hline 60 & 59.926 & 44 & 60.006 & 42 & 60.026 & 38 & 59.942 & 32 & 59.962 & 24 & 59.942 & 18 \\
\hline 60 & 59.966 & 44 & 59.926 & 42 & 59.966 & 38 & 59.982 & 32 & 59.942 & 26 & 60.042 & 20 \\
\hline 60 & 59.986 & 46 & 59.986 & 42 & 59.982 & 38 & 59.982 & 32 & 59.962 & 24 & 60.062 & 20 \\
\hline 60 & 60.006 & 44 & 59.986 & 44 & 60.002 & 38 & 60.022 & 32 & 59.962 & 26 & 60.042 & 22 \\
\hline 60 & 59.986 & 44 & 60.022 & 42 & 59.986 & 38 & 60.042 & 32 & 60.062 & 24 & 60.022 & 20 \\
\hline 60 & 59.906 & 44 & 59.966 & 42 & 59.902 & 38 & 60.022 & 32 & 59.942 & 26 & 60.082 & 20 \\
\hline 60 & 60.006 & 46 & 60.006 & 42 & 59.962 & 38 & 60.022 & 30 & 59.982 & 26 & 60.102 & 20 \\
\hline
\end{tabular}


Table B.5 Angle of Laser Incidence: $10 \mathrm{~m}$ to $100 \mathrm{~m}$

\begin{tabular}{|c|c|c|c|c|c|c|c|c|c|c|c|c|}
\hline \multicolumn{13}{|c|}{$\begin{array}{l}\text { Attic, Bldg. } 226 \\
\text { No Filter } \\
\text { Test Dates: Nov. 1, 5, and 28, } 2001\end{array}$} \\
\hline \multirow{4}{*}{$\begin{array}{c}\text { Distance } \\
\text { (m) }\end{array}$} & \multicolumn{12}{|c|}{ Black } \\
\hline & \multicolumn{12}{|c|}{ Rotation Angle $\left({ }^{\circ}\right)$} \\
\hline & \multicolumn{2}{|c|}{0} & \multicolumn{2}{|c|}{10} & \multicolumn{2}{|c|}{20} & \multicolumn{2}{|l|}{30} & \multicolumn{2}{|c|}{40} & \multicolumn{2}{|c|}{50} \\
\hline & Dist. (m) & Inten. & Dist. (m) & Inten. & Dist. (m) & Inten. & Dist. (m) & Inten. & Dist. (m) & Inten. & Dist. (m) & Inten. \\
\hline 60 & 59.966 & 44 & 60.006 & 42 & 59.942 & 38 & 59.962 & 32 & 59.982 & 26 & 60.062 & 18 \\
\hline 60 & 60.026 & 44 & 59.962 & 42 & 59.982 & 38 & 60.062 & 32 & 60.042 & 24 & 59.962 & 20 \\
\hline 60 & 60.006 & 44 & 59.966 & 42 & 60.002 & 38 & 59.942 & 32 & 59.902 & 26 & 59.922 & 18 \\
\hline 70 & 69.957 & 40 & 70.017 & 38 & 70.037 & 32 & 69.977 & 26 & 69.937 & 20 & 70.057 & 14 \\
\hline 70 & 69.977 & 40 & 69.997 & 38 & 70.017 & 32 & 69.977 & 26 & 70.077 & 20 & 69.937 & 14 \\
\hline 70 & 69.977 & 38 & 69.997 & 36 & 69.997 & 32 & 69.937 & 26 & 69.937 & 20 & 69.897 & 18 \\
\hline 70 & 70.017 & 40 & 70.017 & 38 & 69.997 & 32 & 69.987 & 26 & 69.997 & 20 & 70.237 & 14 \\
\hline 70 & 69.997 & 40 & 69.997 & 36 & 69.997 & 32 & 69.937 & 26 & 69.897 & 20 & 70.137 & 16 \\
\hline 70 & 69.977 & 38 & 69.937 & 38 & 69.977 & 34 & 69.917 & 26 & 69.937 & 20 & 69.957 & 16 \\
\hline 70 & 70.017 & 40 & 69.917 & 38 & 69.957 & 32 & 70.057 & 26 & 70.037 & 22 & 69.977 & 14 \\
\hline 70 & 69.997 & 40 & 69.937 & 38 & 69.977 & 32 & 70.017 & 26 & 69.897 & 20 & 70.077 & 16 \\
\hline 70 & 69.977 & 38 & 69.957 & 38 & 69.937 & 32 & 69.937 & 26 & 69.917 & 20 & 69.917 & 16 \\
\hline 70 & 69.997 & 40 & 69.997 & 36 & 69.937 & 32 & 70.077 & 26 & 69.957 & 18 & 69.997 & 16 \\
\hline 80 & 79.992 & 36 & 79.017 & 34 & 79.932 & 30 & 79.997 & 22 & 79.837 & 16 & 80.017 & 24 \\
\hline 80 & 79.997 & 36 & 79.972 & 34 & 79.932 & 30 & 79.987 & 22 & 79.957 & 16 & 110.057 & 24 \\
\hline 80 & 79.997 & 36 & 79.957 & 34 & 80.017 & 30 & 80.037 & 24 & 80.017 & 16 & 109.857 & 22 \\
\hline 80 & 79.997 & 36 & 79.972 & 34 & 80.017 & 30 & 79.937 & 22 & 79.972 & 16 & 80.137 & 20 \\
\hline 80 & 80.012 & 36 & 79.997 & 34 & 80.032 & 30 & 79.832 & 22 & 80.037 & 14 & 80.112 & 22 \\
\hline 80 & 80.017 & 36 & 80.012 & 34 & 79.977 & 30 & 80.037 & 22 & 80.037 & 16 & 109.817 & 24 \\
\hline 80 & 80.017 & 36 & 79.972 & 36 & 79.997 & 28 & 80.012 & 22 & 79.917 & 16 & 110.077 & 22 \\
\hline 80 & 79.997 & 36 & 79.997 & 34 & 79.977 & 28 & 79.997 & 22 & 80.117 & 16 & 110.037 & 22 \\
\hline 80 & 79.977 & 36 & 79.997 & 34 & 79.932 & 30 & 79.997 & 22 & 80.017 & 16 & 79.997 & 24 \\
\hline 80 & 79.952 & 36 & 79.977 & 34 & 79.977 & 28 & 79.957 & 22 & 80.237 & 16 & 80.177 & 24 \\
\hline 90 & 89.977 & 34 & 89.977 & 32 & 89.957 & 26 & 89.937 & 20 & 90.097 & 24 & 109.297 & 32 \\
\hline 90 & 89.977 & 34 & 89.977 & 32 & 89.977 & 28 & 89.977 & 20 & 89.977 & 24 & 109.337 & 32 \\
\hline 90 & 90.037 & 34 & 89.977 & 32 & 89.997 & 28 & 90.017 & 20 & 90.097 & 24 & 109.317 & 32 \\
\hline 90 & 89.997 & 34 & 89.997 & 32 & 89.897 & 28 & 89.957 & 20 & 90.157 & 24 & 109.377 & 32 \\
\hline 90 & 89.977 & 34 & 89.957 & 32 & 89.857 & 28 & 89.977 & 20 & 90.097 & 24 & 109.317 & 32 \\
\hline 90 & 89.997 & 34 & 90.057 & 32 & 90.057 & 28 & 89.897 & 20 & 90.137 & 24 & 109.257 & 32 \\
\hline 90 & 89.957 & 34 & 89.977 & 32 & 89.937 & 28 & 90.017 & 20 & 90.177 & 24 & 109.437 & 32 \\
\hline 90 & 89.977 & 34 & 89.957 & 32 & 90.037 & 28 & 90.017 & 20 & 90.077 & 24 & 109.277 & 32 \\
\hline 90 & 89.917 & 34 & 89.957 & 32 & 89.997 & 28 & 89.897 & 20 & 90.017 & 24 & 109.397 & 32 \\
\hline 90 & 90.017 & 34 & 89.977 & 32 & 89.997 & 26 & 90.037 & 20 & 90.077 & 22 & 109.397 & 32 \\
\hline 100 & 100.017 & 32 & 100.037 & 30 & 99.997 & 30 & 99.997 & 30 & 100.137 & 32 & 109.257 & 46 \\
\hline 100 & 99.917 & 32 & 100.037 & 30 & 100.057 & 30 & 100.077 & 30 & 100.197 & 32 & 109.277 & 44 \\
\hline 100 & 99.997 & 32 & 100.017 & 30 & 99.917 & 30 & 100.097 & 30 & 100.157 & 32 & 109.237 & 46 \\
\hline 100 & 99.957 & 32 & 99.957 & 32 & 100.057 & 30 & 99.977 & 30 & 100.137 & 32 & 109.257 & 46 \\
\hline 100 & 99.977 & 32 & 99.997 & 32 & 99.977 & 30 & 99.917 & 30 & 100.317 & 32 & 109.317 & 44 \\
\hline 100 & 99.997 & 32 & 99.937 & 32 & 99.937 & 30 & 99.997 & 30 & 109.217 & 32 & 109.257 & 44 \\
\hline 100 & 99.997 & 32 & 100.017 & 32 & 100.017 & 30 & 99.997 & 30 & 100.237 & 32 & 109.237 & 46 \\
\hline 100 & 99.917 & 32 & 99.917 & 32 & 99.937 & 30 & 100.057 & 30 & 100.117 & 32 & 109.277 & 44 \\
\hline 100 & 100.057 & 32 & 99.937 & 32 & 100.097 & 30 & 100.017 & 30 & 100.317 & 32 & 109.277 & 44 \\
\hline 100 & 99.937 & 32 & 99.957 & 32 & 99.997 & 30 & 99.977 & 30 & 100.137 & 32 & 109.277 & 44 \\
\hline
\end{tabular}


Table B.5 Angle of Laser Incidence: $10 \mathrm{~m}$ to $100 \mathrm{~m}$

Attic, Bldg. 226

No Filter

Test Dates: Nov. 1, 5, and 28, 2001

\begin{tabular}{|c|c|c|c|c|c|c|c|c|c|c|}
\hline \multirow{4}{*}{$\begin{array}{c}\text { Distance } \\
(\mathrm{m})\end{array}$} & \multicolumn{10}{|c|}{ Black } \\
\hline & \multicolumn{10}{|c|}{ Rotation Angle $\left({ }^{\circ}\right)$} \\
\hline & \multicolumn{2}{|c|}{60} & \multicolumn{2}{|c|}{70} & \multicolumn{2}{|c|}{80} & \multicolumn{2}{|c|}{85} & \multicolumn{2}{|c|}{90} \\
\hline & Dist. (m) & Inten. & Dist. (m) & Inten. & Dist. (m) & Inten. & Dist. (m) & Inten. & Dist. (m) & Inten. \\
\hline 10 & 10.063 & 38 & 10.063 & 38 & 10.063 & 36 & 10.003 & 42 & 9.702 & 76 \\
\hline 10 & 10.083 & 38 & 10.023 & 38 & 10.063 & 38 & 10.003 & 42 & 9.622 & 76 \\
\hline 10 & 10.063 & 38 & 10.023 & 36 & 10.063 & 38 & 10.003 & 42 & 9.702 & 76 \\
\hline 10 & 10.023 & 38 & 10.043 & 38 & 10.043 & 38 & 10.023 & 42 & 9.582 & 76 \\
\hline 10 & 10.003 & 38 & 10.043 & 38 & 10.083 & 38 & 9.942 & 42 & 9.742 & 76 \\
\hline 10 & 10.043 & 38 & 10.023 & 38 & 10.063 & 38 & 10.043 & 42 & 9.642 & 76 \\
\hline 10 & 10.043 & 38 & 9.962 & 38 & 10.043 & 38 & 10.023 & 42 & 9.762 & 76 \\
\hline 10 & 10.023 & 38 & 10.003 & 38 & 10.103 & 38 & 10.023 & 42 & 9.722 & 76 \\
\hline 10 & 10.023 & 38 & 10.023 & 38 & 10.023 & 38 & 10.063 & 42 & 9.642 & 76 \\
\hline 10 & 10.083 & 38 & 10.043 & 38 & 10.023 & 38 & 9.982 & 42 & 9.722 & 76 \\
\hline 20 & 20.002 & 42 & 20.022 & 40 & 20.062 & 42 & 20.022 & 52 & 19.702 & 70 \\
\hline 20 & 19.962 & 42 & 20.042 & 40 & 20.082 & 42 & 20.002 & 52 & 19.642 & 70 \\
\hline 20 & 20.042 & 42 & 20.062 & 40 & 20.062 & 42 & 20.042 & 52 & 19.662 & 70 \\
\hline 20 & 20.022 & 42 & 20.022 & 40 & 20.082 & 42 & 20.022 & 52 & 19.682 & 70 \\
\hline 20 & 20.042 & 42 & 20.062 & 40 & 20.042 & 42 & 20.022 & 52 & 19.662 & 70 \\
\hline 20 & 19.982 & 42 & 20.002 & 40 & 20.042 & 42 & 20.002 & 52 & 19.622 & 70 \\
\hline 20 & 20.062 & 42 & 20.022 & 40 & 20.022 & 42 & 19.982 & 52 & 19.682 & 70 \\
\hline 20 & 20.022 & 42 & 20.062 & 40 & 20.002 & 42 & 19.982 & 52 & 19.682 & 70 \\
\hline 20 & 20.002 & 42 & 20.022 & 42 & 20.062 & 42 & 19.982 & 52 & 19.662 & 70 \\
\hline 20 & 20.042 & 42 & 20.022 & 40 & 20.102 & 42 & 20.042 & 52 & 19.702 & 70 \\
\hline 30 & 30.011 & 34 & 29.946 & 34 & 30.006 & 50 & 30.046 & 58 & 29.886 & 66 \\
\hline 30 & 30.071 & 34 & 30.011 & 34 & 29.966 & 50 & 30.046 & 58 & 29.886 & 66 \\
\hline 30 & 30.011 & 34 & 30.015 & 34 & 29.966 & 52 & 30.046 & 58 & 29.886 & 66 \\
\hline 30 & 30.011 & 34 & 30.026 & 34 & 29.966 & 50 & 30.086 & 58 & 29.886 & 66 \\
\hline 30 & 30.026 & 34 & 29.966 & 34 & 29.986 & 50 & 30.106 & 58 & 29.886 & 66 \\
\hline 30 & 30.051 & 34 & 30.011 & 34 & 29.986 & 50 & 30.066 & 58 & 29.886 & 66 \\
\hline 30 & 30.026 & 34 & 30.026 & 34 & 30.026 & 50 & 30.091 & 56 & 29.906 & 66 \\
\hline 30 & 30.031 & 34 & 30.111 & 34 & 30.006 & 50 & 30.066 & 58 & 29.906 & 66 \\
\hline 30 & 29.991 & 34 & 29.971 & 34 & 29.946 & 52 & 30.146 & 58 & 29.946 & 66 \\
\hline 30 & 29.966 & 34 & 30.006 & 34 & 29.986 & 50 & 30.126 & 58 & 29.866 & 66 \\
\hline 40 & 40.046 & 28 & 39.986 & 26 & 40.086 & 52 & 40.002 & 54 & 39.842 & 68 \\
\hline 40 & 39.946 & 26 & 39.966 & 26 & 40.146 & 50 & 40.042 & 54 & 39.802 & 68 \\
\hline 40 & 39.946 & 26 & 40.106 & 26 & 40.006 & 50 & 40.106 & 54 & 39.782 & 68 \\
\hline 40 & 40.006 & 28 & 39.966 & 26 & 40.146 & 50 & 40.082 & 54 & 39.762 & 68 \\
\hline 40 & 39.966 & 26 & 39.926 & 26 & 40.062 & 50 & 40.182 & 54 & 39.862 & 68 \\
\hline 40 & 39.966 & 26 & 40.006 & 26 & 40.022 & 50 & 40.162 & 54 & 39.762 & 68 \\
\hline 40 & 40.006 & 26 & 40.102 & 26 & 40.066 & 50 & 40.142 & 54 & 39.762 & 68 \\
\hline 40 & 40.086 & 26 & 40.046 & 26 & 39.982 & 50 & 40.062 & 54 & 39.742 & 70 \\
\hline 40 & 40.006 & 26 & 39.986 & 26 & 39.966 & 52 & 40.142 & 54 & 39.722 & 68 \\
\hline 40 & 39.946 & 26 & 40.046 & 26 & 40.062 & 52 & 40.042 & 54 & 39.842 & 68 \\
\hline 50 & 50.077 & 22 & 50.197 & 50 & 50.237 & 54 & 109.377 & 60 & 109.337 & 74 \\
\hline 50 & 50.157 & 22 & 50.177 & 50 & 50.157 & 54 & 50.317 & 60 & 109.337 & 74 \\
\hline 50 & 50.057 & 20 & 50.157 & 50 & 50.037 & 54 & 50.137 & 60 & 109.337 & 74 \\
\hline 50 & 49.877 & 22 & 50.257 & 50 & 50.057 & 54 & 50.077 & 60 & 109.377 & 74 \\
\hline 50 & 50.037 & 22 & 49.977 & 52 & 50.297 & 54 & 50.237 & 60 & 109.317 & 74 \\
\hline 50 & 50.017 & 22 & 50.137 & 50 & 50.117 & 54 & 50.077 & 60 & 109.277 & 74 \\
\hline 50 & 50.077 & 20 & 50.137 & 50 & 50.317 & 54 & 50.317 & 60 & 109.337 & 74 \\
\hline 50 & 50.057 & 22 & 50.237 & 50 & 50.317 & 54 & 50.257 & 60 & 109.317 & 74 \\
\hline 50 & 50.117 & 22 & 50.217 & 50 & 50.197 & 54 & 50.337 & 60 & 109.317 & 74 \\
\hline 50 & 50.077 & 22 & 50.077 & 50 & 50.177 & 54 & 50.197 & 60 & 109.337 & 74 \\
\hline 60 & 60.062 & 22 & 60.082 & 48 & 109.982 & 66 & 60.142 & 68 & 59.902 & 74 \\
\hline 60 & 60.002 & 22 & 60.342 & 48 & 110.042 & 64 & 59.922 & 68 & 59.642 & 74 \\
\hline 60 & 60.042 & 22 & 60.362 & 48 & 110.022 & 66 & 109.762 & 68 & 59.662 & 74 \\
\hline 60 & 60.122 & 22 & 60.222 & 48 & 109.922 & 66 & 60.102 & 68 & 59.962 & 74 \\
\hline 60 & 60.022 & 22 & 60.282 & 48 & 110.022 & 66 & 60.162 & 68 & 60.042 & 74 \\
\hline 60 & 60.142 & 22 & 60.182 & 48 & 109.962 & 64 & 59.902 & 68 & 59.782 & 74 \\
\hline 60 & 60.022 & 22 & 60.162 & 48 & 110.022 & 66 & 60.122 & 68 & 59.702 & 74 \\
\hline
\end{tabular}


Table B.5 Angle of Laser Incidence: $10 \mathrm{~m}$ to $100 \mathrm{~m}$

Attic, Bldg. 226

No Filter

Test Dates: Nov. 1, 5, and 28, 2001

File: rot_calib_11_01_01.xls

\begin{tabular}{|c|c|c|c|c|c|c|c|c|c|c|}
\hline \multirow{4}{*}{$\begin{array}{c}\text { Distance } \\
\text { (m) }\end{array}$} & \multicolumn{10}{|c|}{ Black } \\
\hline & \multicolumn{10}{|c|}{ Rotation Angle $\left({ }^{\circ}\right)$} \\
\hline & \multicolumn{2}{|c|}{60} & \multicolumn{2}{|c|}{70} & \multicolumn{2}{|c|}{80} & \multicolumn{2}{|c|}{85} & \multicolumn{2}{|c|}{90} \\
\hline & Dist. (m) & Inten. & Dist. (m) & Inten. & Dist. (m) & Inten. & Dist. (m) & Inten. & Dist. (m) & Inten. \\
\hline 60 & 60.082 & 22 & 60.062 & 48 & 109.962 & 66 & 60.182 & 68 & 59.622 & 74 \\
\hline 60 & 60.002 & 20 & 60.222 & 48 & 109.962 & 64 & 60.102 & 68 & 59.762 & 74 \\
\hline 60 & 60.042 & 22 & 60.142 & 48 & 110.122 & 66 & 60.402 & 68 & 59.962 & 74 \\
\hline 70 & 70.037 & 26 & 109.357 & 52 & 109.217 & 62 & 109.177 & 64 & 109.137 & 70 \\
\hline 70 & 70.157 & 26 & 109.377 & 52 & 109.237 & 64 & 109.157 & 64 & 109.137 & 72 \\
\hline 70 & 70.177 & 26 & 109.397 & 52 & 109.217 & 64 & 109.177 & 64 & 109.137 & 70 \\
\hline 70 & 70.097 & 26 & 109.357 & 52 & 109.197 & 62 & 109.177 & 64 & 109.137 & 72 \\
\hline 70 & 69.977 & 26 & 109.417 & 54 & 109.197 & 62 & 109.197 & 64 & 109.177 & 72 \\
\hline 70 & 70.137 & 26 & 109.377 & 54 & 109.217 & 62 & 109.217 & 64 & 109.157 & 70 \\
\hline 70 & 70.277 & 26 & 109.397 & 52 & 109.217 & 62 & 109.197 & 64 & 109.137 & 70 \\
\hline 70 & 70.117 & 26 & 109.397 & 52 & 109.237 & 62 & 109.177 & 64 & 109.137 & 70 \\
\hline 70 & 70.157 & 26 & 109.337 & 52 & 109.197 & 62 & 109.177 & 64 & 109.117 & 70 \\
\hline 70 & 70.057 & 26 & 109.377 & 52 & 109.197 & 62 & 109.197 & 64 & 109.137 & 70 \\
\hline 80 & 111.612 & 40 & 109.977 & 64 & 109.652 & 66 & 109.557 & 68 & 109.277 & 74 \\
\hline 80 & 111.812 & 40 & 109.972 & 64 & 109.792 & 66 & 109.497 & 68 & 109.312 & 72 \\
\hline 80 & 80.177 & 40 & 110.097 & 62 & 109.672 & 66 & 109.622 & 68 & 109.312 & 74 \\
\hline 80 & 111.732 & 40 & 109.952 & 62 & 109.697 & 66 & 109.532 & 68 & 109.312 & 72 \\
\hline 80 & 111.832 & 40 & 110.032 & 62 & 109.692 & 66 & 109.577 & 68 & 109.332 & 72 \\
\hline 80 & 111.837 & 38 & 109.912 & 64 & 109.652 & 66 & 109.612 & 68 & 109.292 & 74 \\
\hline 80 & 111.972 & 40 & 110.012 & 62 & 109.732 & 66 & 109.572 & 68 & 109.312 & 72 \\
\hline 80 & 111.832 & 40 & 109.912 & 62 & 109.732 & 66 & 109.512 & 68 & 109.312 & 74 \\
\hline 80 & 111.852 & 40 & 109.152 & 62 & 109.6923 & 66 & 109.572 & 68 & 109.272 & 72 \\
\hline 80 & 111.637 & 40 & 109.992 & 62 & 109.697 & 66 & 109.572 & 68 & 109.312 & 74 \\
\hline 90 & 109.277 & 52 & 109.157 & 64 & 109.137 & 64 & 109.177 & 68 & 109.137 & 72 \\
\hline 90 & 109.317 & 52 & 109.137 & 62 & 109.077 & 66 & 109.117 & 68 & 109.117 & 72 \\
\hline 90 & 109.297 & 52 & 109.157 & 64 & 109.117 & 66 & 109.097 & 68 & 109.097 & 74 \\
\hline 90 & 109.277 & 52 & 109.157 & 62 & 109.137 & 66 & 109.097 & 68 & 109.117 & 74 \\
\hline 90 & 109.297 & 54 & 109.177 & 62 & 109.157 & 66 & 109.097 & 68 & 109.097 & 72 \\
\hline 90 & 109.277 & 52 & 109.117 & 62 & 109.097 & 66 & 109.137 & 66 & 109.117 & 72 \\
\hline 90 & 109.257 & 52 & 109.177 & 62 & 109.137 & 66 & 109.117 & 68 & 109.097 & 74 \\
\hline 90 & 109.277 & 52 & 109.157 & 62 & 109.117 & 66 & 109.137 & 68 & 109.117 & 72 \\
\hline 90 & 109.217 & 52 & 109.157 & 62 & 109.097 & 66 & 109.117 & 68 & 109.137 & 72 \\
\hline 90 & 109.277 & 54 & 109.197 & 62 & 109.077 & 66 & 109.117 & 68 & 109.097 & 72 \\
\hline 100 & 110.257 & 62 & 109.637 & 66 & 109.457 & 68 & 109.317 & 70 & 109.217 & 74 \\
\hline 100 & 110.197 & 64 & 109.637 & 66 & 109.457 & 68 & 109.337 & 70 & 109.237 & 74 \\
\hline 100 & 110.177 & 62 & 109.737 & 66 & 109.497 & 68 & 109.417 & 70 & 109.237 & 74 \\
\hline 100 & 110.257 & 62 & 109.737 & 66 & 109.437 & 68 & 109.397 & 70 & 109.257 & 74 \\
\hline 100 & 110.177 & 62 & 109.617 & 66 & 109.457 & 68 & 109.357 & 70 & 109.257 & 74 \\
\hline 100 & 110.217 & 62 & 109.637 & 66 & 109.457 & 68 & 109.357 & 70 & 109.217 & 74 \\
\hline 100 & 110.137 & 62 & 109.597 & 66 & 109.497 & 68 & 109.357 & 70 & 109.257 & 74 \\
\hline 100 & 110.177 & 62 & 109.557 & 66 & 109.417 & 68 & 109.317 & 70 & 109.277 & 74 \\
\hline 100 & 110.277 & 62 & 109.677 & 66 & 109.457 & 68 & 109.397 & 70 & 109.237 & 74 \\
\hline 100 & 110.257 & 62 & 109.677 & 66 & 109.457 & 68 & 109.357 & 70 & 109.217 & 74 \\
\hline
\end{tabular}

Table B.6a. Data Set 7: Covariance, $0.045^{\circ}$ Incremental Angle.

\begin{tabular}{|c|c|c|c|c|c|c|c|c|}
\hline \multirow{2}{*}{\multicolumn{9}{|c|}{$\begin{array}{l}\text { Attic, Bldg. } 226 \\
\text { Test date: Sept. 03, } 2002\end{array}$}} \\
\hline & & & & & & & & \\
\hline \multicolumn{3}{|c|}{ Strip 1} & \multicolumn{3}{|c|}{ Strip 2} & \multicolumn{3}{|c|}{ Strip 3} \\
\hline Range (m) & $\phi\left({ }^{\circ}\right)$ & $\theta\left({ }^{\circ}\right)$ & Range (m) & $\phi\left({ }^{\circ}\right)$ & $\theta\left({ }^{\circ}\right)$ & Range (m) & $\phi\left({ }^{\circ}\right)$ & $\theta\left({ }^{\circ}\right)$ \\
\hline 9.999 & 89.46 & 39.6 & 9.999 & 89.46 & 39.645 & 9.958 & 89.46 & 39.69 \\
\hline 9.999 & 89.46 & 39.6 & 10.019 & 89.46 & 39.645 & 9.958 & 89.46 & 39.69 \\
\hline
\end{tabular}


Table B.6a. Data Set 7: Covariance, $0.045^{\circ}$ Incremental Angle.

\begin{tabular}{|c|c|c|c|c|c|c|c|c|}
\hline \multirow{2}{*}{\multicolumn{3}{|c|}{$\begin{array}{l}\text { Attic, Bldg. } 226 \\
\text { Test date: Sept. 03, } 2002\end{array}$}} & & & & \multicolumn{3}{|c|}{ File: dataset7_covariance_10m_045.xls } \\
\hline & & & & Strip 2 & & & Strip 3 & \\
\hline Range (m) & $\phi\left({ }^{0}\right)$ & $\theta\left(^{0}\right)$ & Range (m) & $\phi\left({ }^{0}\right)$ & $\theta\left(^{\circ}\right)$ & Range (m) & $\phi\left({ }^{0}\right)$ & $\theta\left({ }^{0}\right)$ \\
\hline 10.019 & 89.46 & 39.6 & 9.978 & 89.46 & 39.645 & 10.019 & 89.46 & 39.69 \\
\hline 9.978 & 89.46 & 39.6 & 10.039 & 89.46 & 39.645 & 10.019 & 89.46 & 39.69 \\
\hline 10.019 & 89.46 & 39.6 & 9.958 & 89.46 & 39.645 & 9.978 & 89.46 & 39.69 \\
\hline 9.995 & 89.46 & 39.6 & 9.999 & 89.46 & 39.645 & 10.019 & 89.46 & 39.69 \\
\hline 9.954 & 89.46 & 39.6 & 9.974 & 89.46 & 39.645 & 10.019 & 89.46 & 39.69 \\
\hline 10.015 & 89.46 & 39.6 & 9.995 & 89.46 & 39.645 & 10.039 & 89.46 & 39.69 \\
\hline 9.974 & 89.46 & 39.6 & 9.974 & 89.46 & 39.645 & 9.978 & 89.46 & 39.69 \\
\hline 9.954 & 89.46 & 39.6 & 9.974 & 89.46 & 39.645 & 10.019 & 89.46 & 39.69 \\
\hline 10.019 & 89.505 & 39.6 & 9.978 & 89.505 & 39.645 & 9.999 & 89.505 & 39.69 \\
\hline 9.999 & 89.505 & 39.6 & 9.999 & 89.505 & 39.645 & 9.958 & 89.505 & 39.69 \\
\hline 9.978 & 89.505 & 39.6 & 9.999 & 89.505 & 39.645 & 9.958 & 89.505 & 39.69 \\
\hline 9.999 & 89.505 & 39.6 & 10.039 & 89.505 & 39.645 & 9.978 & 89.505 & 39.69 \\
\hline 9.958 & 89.505 & 39.6 & 9.978 & 89.505 & 39.645 & 10.019 & 89.505 & 39.69 \\
\hline 10.019 & 89.505 & 39.6 & 10.019 & 89.505 & 39.645 & 10.019 & 89.505 & 39.69 \\
\hline 9.999 & 89.505 & 39.6 & 9.999 & 89.505 & 39.645 & 9.978 & 89.505 & 39.69 \\
\hline 9.958 & 89.505 & 39.6 & 9.999 & 89.505 & 39.645 & 9.999 & 89.505 & 39.69 \\
\hline 10.019 & 89.505 & 39.6 & 9.978 & 89.505 & 39.645 & 9.999 & 89.505 & 39.69 \\
\hline 9.999 & 89.505 & 39.6 & 9.978 & 89.505 & 39.645 & 9.978 & 89.505 & 39.69 \\
\hline 9.999 & 89.55 & 39.6 & 10.039 & 89.55 & 39.645 & 10.019 & 89.55 & 39.69 \\
\hline 10.019 & 89.55 & 39.6 & 9.999 & 89.55 & 39.645 & 10.019 & 89.55 & 39.69 \\
\hline 9.999 & 89.55 & 39.6 & 9.999 & 89.55 & 39.645 & 9.999 & 89.55 & 39.69 \\
\hline 10.019 & 89.55 & 39.6 & 10.019 & 89.55 & 39.645 & 9.978 & 89.55 & 39.69 \\
\hline 10.015 & 89.55 & 39.6 & 9.999 & 89.55 & 39.645 & 10.019 & 89.55 & 39.69 \\
\hline 9.995 & 89.55 & 39.6 & 9.999 & 89.55 & 39.645 & 9.974 & 89.55 & 39.69 \\
\hline 10.015 & 89.55 & 39.6 & 10.015 & 89.55 & 39.645 & 9.995 & 89.55 & 39.69 \\
\hline 9.995 & 89.55 & 39.6 & 10.015 & 89.55 & 39.645 & 10.015 & 89.55 & 39.69 \\
\hline 10.015 & 89.55 & 39.6 & 10.015 & 89.55 & 39.645 & 10.015 & 89.55 & 39.69 \\
\hline 10.015 & 89.55 & 39.6 & 10.015 & 89.55 & 39.645 & 10.015 & 89.55 & 39.69 \\
\hline 9.958 & 89.595 & 39.6 & 10.019 & 89.595 & 39.645 & 10.019 & 89.595 & 39.699 \\
\hline 9.999 & 89.595 & 39.6 & 9.958 & 89.595 & 39.645 & 9.999 & 89.595 & 39.699 \\
\hline 9.978 & 89.595 & 39.6 & 9.999 & 89.595 & 39.645 & 9.999 & 89.595 & 39.69 \\
\hline 9.978 & 89.595 & 39.6 & 9.958 & 89.595 & 39.645 & 9.999 & 89.595 & 39.69 \\
\hline 9.978 & 89.595 & 39.6 & 9.999 & 89.595 & 39.645 & 9.999 & 89.595 & 39.69 \\
\hline 10.039 & 89.595 & 39.6 & 10.019 & 89.595 & 39.645 & 9.999 & 89.595 & 39.69 \\
\hline 9.999 & 89.595 & 39.6 & 9.999 & 89.595 & 39.645 & 9.999 & 89.595 & 39.69 \\
\hline 9.978 & 89.595 & 39.6 & 9.978 & 89.595 & 39.645 & 10.039 & 89.595 & 39.69 \\
\hline 10.019 & 89.595 & 39.6 & 9.978 & 89.595 & 39.645 & 9.978 & 89.595 & 39.69 \\
\hline 9.999 & 89.595 & 39.6 & 9.999 & 89.595 & 39.645 & 9.999 & 89.595 & 39.69 \\
\hline 9.978 & 89.64 & 39.6 & 9.978 & 89.64 & 39.645 & 9.958 & 89.64 & 39.699 \\
\hline 10.039 & 89.64 & 39.6 & 9.999 & 89.64 & 39.645 & 9.978 & 89.64 & 39.699 \\
\hline 9.999 & 89.64 & 39.6 & 10.019 & 89.64 & 39.645 & 10.039 & 89.64 & 39.699 \\
\hline 10.019 & 89.64 & 39.6 & 9.999 & 89.64 & 39.645 & 10.019 & 89.64 & 39.699 \\
\hline 9.978 & 89.64 & 39.6 & 10.015 & 89.64 & 39.645 & 9.978 & 89.64 & 39.699 \\
\hline 9.978 & 89.64 & 39.6 & 9.954 & 89.64 & 39.645 & 10.039 & 89.64 & 39.699 \\
\hline 9.999 & 89.64 & 39.6 & 10.015 & 89.64 & 39.645 & 10.019 & 89.64 & 39.699 \\
\hline 9.978 & 89.64 & 39.6 & 9.995 & 89.64 & 39.645 & 9.978 & 89.64 & 39.699 \\
\hline 10.035 & 89.64 & 39.6 & 10.015 & 89.64 & 39.645 & 9.999 & 89.64 & 39.699 \\
\hline 10.015 & 89.64 & 39.6 & 10.035 & 89.64 & 39.645 & 10.019 & 89.64 & 39.699 \\
\hline 10.019 & 89.685 & 39.6 & 10.019 & 89.685 & 39.645 & 9.999 & 89.685 & 39.699 \\
\hline 9.999 & 89.685 & 39.6 & 9.999 & 89.685 & 39.645 & 9.999 & 89.685 & 39.699 \\
\hline
\end{tabular}


Table B.6a. Data Set 7: Covariance, $0.045^{\circ}$ Incremental Angle.

\begin{tabular}{|c|c|c|c|c|c|c|c|c|}
\hline \multirow{2}{*}{\multicolumn{3}{|c|}{\begin{tabular}{|l|} 
Attic, Bldg. 226 \\
Test date: Sept. 03, 2002 \\
\end{tabular}}} & & & & \multicolumn{3}{|c|}{ File: dataset7_covariance_10m_045.xls } \\
\hline & & & \multicolumn{3}{|c|}{ Strip 2} & \multicolumn{3}{|c|}{ Strip 3} \\
\hline Range (m) & $\phi\left({ }^{\circ}\right)$ & $\theta\left({ }^{\circ}\right)$ & Range (m) & $\phi\left({ }^{\circ}\right)$ & $\theta\left({ }^{\circ}\right)$ & Range (m) & $\phi\left({ }^{\circ}\right)$ & $\theta\left({ }^{\circ}\right)$ \\
\hline 10.019 & 89.685 & 39.6 & 9.999 & 89.685 & 39.645 & 9.978 & 89.685 & 39.699 \\
\hline 9.999 & 89.685 & 39.6 & 9.978 & 89.685 & 39.645 & 9.954 & 89.685 & 39.699 \\
\hline 10.015 & 89.685 & 39.6 & 9.999 & 89.685 & 39.645 & 9.995 & 89.685 & 39.699 \\
\hline 9.995 & 89.685 & 39.6 & 10.019 & 89.685 & 39.645 & 9.954 & 89.685 & 39.699 \\
\hline 9.974 & 89.685 & 39.6 & 9.999 & 89.685 & 39.645 & 9.974 & 89.685 & 39.699 \\
\hline 10.015 & 89.685 & 39.6 & 9.999 & 89.685 & 39.645 & 10.015 & 89.685 & 39.699 \\
\hline 9.954 & 89.685 & 39.6 & 9.999 & 89.685 & 39.645 & 10.015 & 89.685 & 39.699 \\
\hline 9.954 & 89.685 & 39.6 & 9.999 & 89.685 & 39.645 & 10.035 & 89.685 & 39.699 \\
\hline 9.999 & 89.73 & 39.6 & 10.019 & 89.73 & 39.645 & 9.958 & 89.73 & 39.699 \\
\hline 10.039 & 89.73 & 39.6 & 9.958 & 89.73 & 39.645 & 10.019 & 89.73 & 39.699 \\
\hline 9.999 & 89.73 & 39.6 & 10.019 & 89.73 & 39.645 & 10.019 & 89.73 & 39.699 \\
\hline 10.019 & 89.73 & 39.6 & 9.999 & 89.73 & 39.645 & 10.019 & 89.73 & 39.699 \\
\hline 9.999 & 89.73 & 39.6 & 9.999 & 89.73 & 39.645 & 9.978 & 89.73 & 39.699 \\
\hline 9.978 & 89.73 & 39.6 & 10.019 & 89.73 & 39.645 & 10.019 & 89.73 & 39.699 \\
\hline 10.019 & 89.73 & 39.6 & 10.019 & 89.73 & 39.645 & 10.019 & 89.73 & 39.699 \\
\hline 9.999 & 89.73 & 39.6 & 9.999 & 89.73 & 39.645 & 9.978 & 89.73 & 39.699 \\
\hline 10.015 & 89.73 & 39.6 & 9.999 & 89.73 & 39.645 & 9.978 & 89.73 & 39.699 \\
\hline 10.015 & 89.73 & 39.6 & 10.015 & 89.73 & 39.645 & 10.015 & 89.73 & 39.699 \\
\hline 9.978 & 89.775 & 39.6 & 10.019 & 89.775 & 39.645 & 9.999 & 89.775 & 39.699 \\
\hline 10.019 & 89.775 & 39.6 & 9.999 & 89.775 & 39.645 & 9.978 & 89.775 & 39.699 \\
\hline 10.019 & 89.775 & 39.6 & 10.019 & 89.775 & 39.645 & 10.019 & 89.775 & 39.699 \\
\hline 10.019 & 89.775 & 39.6 & 10.019 & 89.775 & 39.645 & 9.999 & 89.775 & 39.699 \\
\hline 9.978 & 89.775 & 39.6 & 9.999 & 89.775 & 39.645 & 10.019 & 89.775 & 39.699 \\
\hline 9.978 & 89.775 & 39.6 & 10.019 & 89.775 & 39.645 & 9.999 & 89.775 & 39.699 \\
\hline 9.999 & 89.775 & 39.6 & 10.039 & 89.775 & 39.645 & 10.019 & 89.775 & 39.699 \\
\hline 9.958 & 89.775 & 39.6 & 9.999 & 89.775 & 39.645 & 10.015 & 89.775 & 39.699 \\
\hline 9.995 & 89.775 & 39.6 & 9.999 & 89.775 & 39.645 & 10.015 & 89.775 & 39.699 \\
\hline 9.995 & 89.775 & 39.6 & 9.995 & 89.775 & 39.645 & 10.015 & 89.775 & 39.699 \\
\hline 9.958 & 89.82 & 39.6 & 9.999 & 89.82 & 39.645 & 10.019 & 89.82 & 39.699 \\
\hline 10.019 & 89.82 & 39.6 & 10.019 & 89.82 & 39.645 & 9.999 & 89.82 & 39.699 \\
\hline 9.978 & 89.82 & 39.6 & 9.978 & 89.82 & 39.645 & 9.958 & 89.82 & 39.699 \\
\hline 9.999 & 89.82 & 39.6 & 9.978 & 89.82 & 39.645 & 9.958 & 89.82 & 39.699 \\
\hline 9.978 & 89.82 & 39.6 & 9.978 & 89.82 & 39.645 & 9.999 & 89.82 & 39.699 \\
\hline 10.019 & 89.82 & 39.6 & 9.999 & 89.82 & 39.645 & 10.019 & 89.82 & 39.699 \\
\hline 9.999 & 89.82 & 39.6 & 9.958 & 89.82 & 39.645 & 10.039 & 89.82 & 39.699 \\
\hline 9.958 & 89.82 & 39.6 & 9.958 & 89.82 & 39.645 & 9.999 & 89.82 & 39.699 \\
\hline 9.999 & 89.82 & 39.6 & 9.999 & 89.82 & 39.645 & 9.978 & 89.82 & 39.699 \\
\hline 9.999 & 89.82 & 39.6 & 9.958 & 89.82 & 39.645 & 10.015 & 89.82 & 39.699 \\
\hline 9.999 & 89.865 & 39.6 & 9.999 & 89.865 & 39.645 & 10.019 & 89.865 & 39.699 \\
\hline 9.978 & 89.865 & 39.6 & 9.978 & 89.865 & 39.645 & 10.019 & 89.865 & 39.699 \\
\hline 9.999 & 89.865 & 39.6 & 9.999 & 89.865 & 39.645 & 9.999 & 89.865 & 39.699 \\
\hline 10.019 & 89.865 & 39.6 & 9.999 & 89.865 & 39.645 & 9.958 & 89.865 & 39.699 \\
\hline 9.978 & 89.865 & 39.6 & 10.019 & 89.865 & 39.645 & 9.995 & 89.865 & 39.699 \\
\hline 9.954 & 89.865 & 39.6 & 9.999 & 89.865 & 39.645 & 9.954 & 89.865 & 39.69 \\
\hline 9.995 & 89.865 & 39.6 & 9.999 & 89.865 & 39.645 & 9.974 & 89.865 & 39.69 \\
\hline 9.995 & 89.865 & 39.6 & 9.999 & 89.865 & 39.645 & 9.995 & 89.865 & 39.69 \\
\hline 10.015 & 89.865 & 39.6 & 9.999 & 89.865 & 39.645 & 10.015 & 89.865 & 39.69 \\
\hline 10.015 & 89.865 & 39.6 & 9.999 & 89.865 & 39.645 & 10.015 & 89.865 & 39.69 \\
\hline 9.978 & 89.91 & 39.6 & 9.958 & 89.91 & 39.645 & 9.999 & 89.91 & 39.69 \\
\hline 9.999 & 89.91 & 39.6 & 10.019 & 89.91 & 39.645 & 9.978 & 89.91 & 39.69 \\
\hline
\end{tabular}


Table B.6a. Data Set 7: Covariance, $0.045^{\circ}$ Incremental Angle.

\begin{tabular}{|c|c|c|c|c|c|c|c|c|}
\hline \multirow{2}{*}{\multicolumn{3}{|c|}{$\begin{array}{l}\text { Attic, Bldg. } 226 \\
\text { Test date: Sept. 03, } 2002\end{array}$}} & & & & \multicolumn{3}{|c|}{ File: dataset7_covariance_10m_045.xls } \\
\hline & & & \multicolumn{3}{|c|}{ Strip 2} & \multicolumn{3}{|c|}{ Strip 3} \\
\hline Range (m) & $\phi\left({ }^{\circ}\right)$ & $\theta\left({ }^{\circ}\right)$ & Range (m) & $\phi\left({ }^{\circ}\right)$ & $\theta\left({ }^{\circ}\right)$ & Range (m) & $\phi\left({ }^{\circ}\right)$ & $\theta\left({ }^{\circ}\right)$ \\
\hline 9.978 & 89.91 & 39.6 & 9.958 & 89.91 & 39.645 & 9.978 & 89.91 & 39.69 \\
\hline 10.019 & 89.91 & 39.6 & 10.019 & 89.91 & 39.645 & 9.999 & 89.91 & 39.69 \\
\hline 10.019 & 89.91 & 39.6 & 9.999 & 89.91 & 39.645 & 9.999 & 89.91 & 39.69 \\
\hline 9.999 & 89.91 & 39.6 & 9.958 & 89.91 & 39.645 & 10.019 & 89.91 & 39.69 \\
\hline 9.978 & 89.91 & 39.6 & 9.958 & 89.91 & 39.645 & 10.019 & 89.91 & 39.69 \\
\hline 10.019 & 89.91 & 39.6 & 9.958 & 89.91 & 39.645 & 9.999 & 89.91 & 39.69 \\
\hline 9.999 & 89.91 & 39.6 & 10.019 & 89.91 & 39.645 & 9.999 & 89.91 & 39.69 \\
\hline 9.978 & 89.91 & 39.6 & 10.015 & 89.91 & 39.645 & 10.019 & 89.91 & 39.69 \\
\hline 9.978 & 89.955 & 39.6 & 10.019 & 89.955 & 39.645 & 9.978 & 89.955 & 39.69 \\
\hline 10.019 & 89.955 & 39.6 & 9.978 & 89.955 & 39.645 & 9.999 & 89.955 & 39.69 \\
\hline 9.958 & 89.955 & 39.6 & 10.019 & 89.955 & 39.645 & 9.978 & 89.955 & 39.69 \\
\hline 9.999 & 89.955 & 39.6 & 9.978 & 89.955 & 39.645 & 10.019 & 89.955 & 39.69 \\
\hline 10.039 & 89.955 & 39.6 & 9.999 & 89.955 & 39.645 & 9.958 & 89.955 & 39.69 \\
\hline 9.999 & 89.955 & 39.6 & 10.019 & 89.955 & 39.645 & 10.019 & 89.955 & 39.69 \\
\hline 9.958 & 89.955 & 39.6 & 10.019 & 89.955 & 39.645 & 10.019 & 89.955 & 39.69 \\
\hline 10.019 & 89.955 & 39.6 & 10.019 & 89.955 & 39.645 & 9.978 & 89.955 & 39.69 \\
\hline 9.999 & 89.955 & 39.6 & 9.999 & 89.955 & 39.645 & 9.974 & 89.955 & 39.69 \\
\hline 9.954 & 89.955 & 39.6 & 10.019 & 89.955 & 39.645 & 9.995 & 89.955 & 39.69 \\
\hline 9.958 & 90 & 39.6 & 9.978 & 90 & 39.645 & 9.978 & 90 & 39.69 \\
\hline 9.999 & 90 & 39.6 & 10.019 & 90 & 39.645 & 9.958 & 90 & 39.69 \\
\hline 10.039 & 90 & 39.6 & 9.999 & 90 & 39.645 & 10.039 & 90 & 39.69 \\
\hline 9.978 & 90 & 39.6 & 9.978 & 90 & 39.645 & 10.039 & 90 & 39.69 \\
\hline 10.019 & 90 & 39.6 & 9.978 & 90 & 39.645 & 9.978 & 90 & 39.69 \\
\hline 9.999 & 90 & 39.6 & 10.019 & 90 & 39.645 & 10.019 & 90 & 39.69 \\
\hline 9.958 & 90 & 39.6 & 10.019 & 90 & 39.645 & 10.019 & 90 & 39.69 \\
\hline 10.019 & 90 & 39.6 & 10.019 & 90 & 39.645 & 10.039 & 90 & 39.69 \\
\hline 9.938 & 90 & 39.6 & 9.974 & 90 & 39.645 & 9.978 & 90 & 39.69 \\
\hline 10.019 & 90 & 39.6 & 9.995 & 90 & 39.645 & 10.019 & 90 & 39.69 \\
\hline 9.999 & 90.045 & 39.6 & 9.999 & 90.045 & 39.645 & 10.019 & 90.045 & 39.69 \\
\hline 10.019 & 90.045 & 39.6 & 9.958 & 90.045 & 39.645 & 9.999 & 90.045 & 39.699 \\
\hline 9.978 & 90.045 & 39.6 & 10.019 & 90.045 & 39.645 & 9.978 & 90.045 & 39.69 \\
\hline 9.999 & 90.045 & 39.6 & 9.978 & 90.045 & 39.645 & 9.978 & 90.045 & 39.69 \\
\hline 9.958 & 90.045 & 39.6 & 9.999 & 90.045 & 39.645 & 9.999 & 90.045 & 39.69 \\
\hline 10.019 & 90.045 & 39.6 & 10.019 & 90.045 & 39.645 & 10.019 & 90.045 & 39.69 \\
\hline 10.019 & 90.045 & 39.6 & 9.999 & 90.045 & 39.645 & 9.999 & 90.045 & 39.69 \\
\hline 9.958 & 90.045 & 39.6 & 9.999 & 90.045 & 39.645 & 9.978 & 90.045 & 39.69 \\
\hline 9.995 & 90.045 & 39.6 & 10.019 & 90.045 & 39.645 & 9.974 & 90.045 & 39.69 \\
\hline 9.954 & 90.045 & 39.6 & 10.019 & 90.045 & 39.645 & 10.015 & 90.045 & 39.69 \\
\hline 10.019 & 90.09 & 39.6 & 9.978 & 90.09 & 39.645 & 9.999 & 90.09 & 39.699 \\
\hline 10.019 & 90.09 & 39.6 & 9.958 & 90.09 & 39.645 & 10.019 & 90.09 & 39.699 \\
\hline 9.999 & 90.09 & 39.6 & 10.019 & 90.09 & 39.645 & 9.999 & 90.09 & 39.699 \\
\hline 10.019 & 90.09 & 39.6 & 9.999 & 90.09 & 39.645 & 9.999 & 90.09 & 39.699 \\
\hline 9.958 & 90.09 & 39.6 & 10.019 & 90.09 & 39.645 & 10.019 & 90.09 & 39.699 \\
\hline 10.019 & 90.09 & 39.6 & 10.019 & 90.09 & 39.645 & 9.978 & 90.09 & 39.699 \\
\hline 10.019 & 90.09 & 39.6 & 10.015 & 90.09 & 39.645 & 9.978 & 90.09 & 39.699 \\
\hline 9.958 & 90.09 & 39.6 & 10.015 & 90.09 & 39.645 & 10.019 & 90.09 & 39.69 \\
\hline 9.999 & 90.09 & 39.6 & 10.015 & 90.09 & 39.645 & 9.978 & 90.09 & 39.69 \\
\hline 9.978 & 90.09 & 39.6 & 10.015 & 90.09 & 39.645 & 9.958 & 90.09 & 39.69 \\
\hline 10.019 & 90.135 & 39.6 & 10.019 & 90.135 & 39.645 & 10.019 & 90.135 & 39.699 \\
\hline 10.019 & 90.135 & 39.6 & 10.019 & 90.135 & 39.645 & 10.019 & 90.135 & 39.699 \\
\hline
\end{tabular}


Table B.6a. Data Set 7: Covariance, $0.045^{\circ}$ Incremental Angle.

\begin{tabular}{|c|c|c|c|c|c|c|c|c|}
\hline \multirow{2}{*}{\multicolumn{3}{|c|}{\begin{tabular}{|l|} 
Attic, Bldg. 226 \\
Test date: Sept. 03, 2002 \\
\end{tabular}}} & & & & \multicolumn{3}{|c|}{ File: dataset7_covariance_10m_045.xls } \\
\hline & & & \multicolumn{3}{|c|}{ Strip 2} & \multicolumn{3}{|c|}{ Strip 3} \\
\hline Range (m) & $\phi\left({ }^{\circ}\right)$ & $\theta\left({ }^{\circ}\right)$ & Range (m) & $\phi\left({ }^{\circ}\right)$ & $\theta\left({ }^{\circ}\right)$ & Range (m) & $\phi\left({ }^{\circ}\right)$ & $\theta\left({ }^{\circ}\right)$ \\
\hline 9.978 & 90.135 & 39.6 & 10.019 & 90.135 & 39.645 & 9.958 & 90.135 & 39.699 \\
\hline 9.999 & 90.135 & 39.6 & 10.019 & 90.135 & 39.645 & 9.999 & 90.135 & 39.699 \\
\hline 10.019 & 90.135 & 39.6 & 10.019 & 90.135 & 39.645 & 10.019 & 90.135 & 39.699 \\
\hline 10.019 & 90.135 & 39.6 & 10.019 & 90.135 & 39.645 & 9.978 & 90.135 & 39.699 \\
\hline 10.019 & 90.135 & 39.6 & 10.019 & 90.135 & 39.645 & 9.938 & 90.135 & 39.699 \\
\hline 10.019 & 90.135 & 39.6 & 10.019 & 90.135 & 39.645 & 10.019 & 90.135 & 39.699 \\
\hline 9.978 & 90.135 & 39.6 & 10.019 & 90.135 & 39.645 & 9.999 & 90.135 & 39.699 \\
\hline 9.978 & 90.135 & 39.6 & 10.019 & 90.135 & 39.645 & 9.995 & 90.135 & 39.699 \\
\hline 9.999 & 90.18 & 39.6 & 10.019 & 90.18 & 39.645 & 10.019 & 90.18 & 39.699 \\
\hline 10.019 & 90.18 & 39.6 & 9.958 & 90.18 & 39.645 & 9.999 & 90.18 & 39.699 \\
\hline 9.958 & 90.18 & 39.6 & 10.019 & 90.18 & 39.645 & 9.958 & 90.18 & 39.699 \\
\hline 9.999 & 90.18 & 39.6 & 10.019 & 90.18 & 39.645 & 9.958 & 90.18 & 39.699 \\
\hline 10.019 & 90.18 & 39.6 & 10.019 & 90.18 & 39.645 & 9.999 & 90.18 & 39.699 \\
\hline 10.019 & 90.18 & 39.6 & 10.035 & 90.18 & 39.645 & 9.978 & 90.18 & 39.699 \\
\hline 9.978 & 90.18 & 39.6 & 10.015 & 90.18 & 39.645 & 10.019 & 90.18 & 39.699 \\
\hline 10.019 & 90.18 & 39.6 & 9.954 & 90.18 & 39.645 & 9.999 & 90.18 & 39.699 \\
\hline 9.978 & 90.18 & 39.6 & 10.035 & 90.18 & 39.645 & 9.999 & 90.18 & 39.699 \\
\hline 9.958 & 90.18 & 39.6 & 10.015 & 90.18 & 39.645 & 9.999 & 90.18 & 39.699 \\
\hline 9.978 & 90.225 & 39.6 & 9.999 & 90.225 & 39.645 & 9.978 & 90.225 & 39.699 \\
\hline 10.019 & 90.225 & 39.6 & 9.999 & 90.225 & 39.645 & 9.999 & 90.225 & 39.699 \\
\hline 10.019 & 90.225 & 39.6 & 9.999 & 90.225 & 39.645 & 10.019 & 90.225 & 39.699 \\
\hline 9.978 & 90.225 & 39.6 & 9.978 & 90.225 & 39.645 & 9.999 & 90.225 & 39.699 \\
\hline 9.999 & 90.225 & 39.6 & 9.999 & 90.225 & 39.645 & 10.019 & 90.225 & 39.699 \\
\hline 9.978 & 90.225 & 39.6 & 9.999 & 90.225 & 39.645 & 9.999 & 90.225 & 39.699 \\
\hline 10.019 & 90.225 & 39.6 & 10.019 & 90.225 & 39.645 & 9.999 & 90.225 & 39.699 \\
\hline 9.999 & 90.225 & 39.6 & 10.019 & 90.225 & 39.645 & 9.999 & 90.225 & 39.699 \\
\hline 10.035 & 90.225 & 39.6 & 9.999 & 90.225 & 39.645 & 9.999 & 90.225 & 39.699 \\
\hline 9.974 & 90.225 & 39.6 & 10.019 & 90.225 & 39.645 & 9.974 & 90.225 & 39.699 \\
\hline 9.999 & 90.27 & 39.6 & 9.999 & 90.27 & 39.645 & 10.019 & 90.27 & 39.699 \\
\hline 9.958 & 90.27 & 39.6 & 9.958 & 90.27 & 39.645 & 10.019 & 90.27 & 39.699 \\
\hline 9.958 & 90.27 & 39.6 & 9.999 & 90.27 & 39.645 & 9.978 & 90.27 & 39.699 \\
\hline 9.999 & 90.27 & 39.6 & 9.999 & 90.27 & 39.645 & 9.958 & 90.27 & 39.699 \\
\hline 10.019 & 90.27 & 39.6 & 9.995 & 90.27 & 39.645 & 9.978 & 90.27 & 39.699 \\
\hline 9.999 & 90.27 & 39.6 & 10.015 & 90.27 & 39.645 & 10.019 & 90.27 & 39.699 \\
\hline 9.999 & 90.27 & 39.6 & 10.015 & 90.27 & 39.645 & 9.978 & 90.27 & 39.699 \\
\hline 10.019 & 90.27 & 39.6 & 10.015 & 90.27 & 39.645 & 9.958 & 90.27 & 39.699 \\
\hline 9.999 & 90.27 & 39.6 & 10.015 & 90.27 & 39.645 & 9.978 & 90.27 & 39.699 \\
\hline 9.999 & 90.27 & 39.6 & 10.015 & 90.27 & 39.645 & 10.019 & 90.27 & 39.699 \\
\hline 9.999 & 90.315 & 39.6 & 9.999 & 90.315 & 39.645 & 10.019 & 90.315 & 39.699 \\
\hline 10.019 & 90.315 & 39.6 & 9.978 & 90.315 & 39.645 & 9.999 & 90.315 & 39.699 \\
\hline 9.978 & 90.315 & 39.6 & 10.019 & 90.315 & 39.645 & 10.019 & 90.315 & 39.699 \\
\hline 9.958 & 90.315 & 39.6 & 10.019 & 90.315 & 39.645 & 10.019 & 90.315 & 39.699 \\
\hline 10.015 & 90.315 & 39.6 & 10.019 & 90.315 & 39.645 & 9.978 & 90.315 & 39.699 \\
\hline 9.999 & 90.315 & 39.6 & 9.978 & 90.315 & 39.645 & 10.019 & 90.315 & 39.699 \\
\hline 9.974 & 90.315 & 39.6 & 10.059 & 90.315 & 39.645 & 10.015 & 90.315 & 39.699 \\
\hline 10.015 & 90.315 & 39.6 & 9.999 & 90.315 & 39.645 & 9.974 & 90.315 & 39.699 \\
\hline 9.974 & 90.315 & 39.6 & 10.015 & 90.315 & 39.645 & 9.995 & 90.315 & 39.699 \\
\hline 10.015 & 90.315 & 39.6 & 9.974 & 90.315 & 39.645 & 10.015 & 90.315 & 39.699 \\
\hline 9.958 & 90.36 & 39.6 & 10.019 & 90.36 & 39.645 & 9.999 & 90.36 & 39.699 \\
\hline 10.019 & 90.36 & 39.6 & 9.999 & 90.36 & 39.645 & 9.978 & 90.36 & 39.699 \\
\hline
\end{tabular}


Table B.6a. Data Set 7: Covariance, $0.045^{\circ}$ Incremental Angle.

\begin{tabular}{|c|c|c|c|c|c|c|c|c|}
\hline \multirow{2}{*}{\multicolumn{3}{|c|}{$\begin{array}{l}\text { Attic, Bldg. } 226 \\
\text { Test date: Sept. 03, } 2002\end{array}$}} & & & & \multicolumn{3}{|c|}{ File: dataset7_covariance_10m_045.xls } \\
\hline & & & & Strip 2 & & & Strip 3 & \\
\hline Range (m) & $\phi\left({ }^{0}\right)$ & $\theta\left({ }^{\circ}\right)$ & Range (m) & $\phi\left({ }^{\circ}\right)$ & $\theta\left({ }^{\circ}\right)$ & Range (m) & $\phi\left({ }^{0}\right)$ & $\theta\left({ }^{\circ}\right)$ \\
\hline 10.019 & 90.36 & 39.6 & 9.978 & 90.36 & 39.645 & 10.019 & 90.36 & 39.699 \\
\hline 10.019 & 90.36 & 39.6 & 10.019 & 90.36 & 39.645 & 10.019 & 90.36 & 39.699 \\
\hline 9.978 & 90.36 & 39.6 & 9.978 & 90.36 & 39.645 & 9.999 & 90.36 & 39.699 \\
\hline 9.978 & 90.36 & 39.6 & 9.999 & 90.36 & 39.645 & 9.999 & 90.36 & 39.699 \\
\hline 10.039 & 90.36 & 39.6 & 9.958 & 90.36 & 39.645 & 9.958 & 90.36 & 39.699 \\
\hline 10.019 & 90.36 & 39.6 & 9.999 & 90.36 & 39.645 & 10.019 & 90.36 & 39.699 \\
\hline 10.019 & 90.36 & 39.6 & 9.978 & 90.36 & 39.645 & 10.019 & 90.36 & 39.699 \\
\hline 10.015 & 90.36 & 39.6 & 9.995 & 90.36 & 39.645 & 10.019 & 90.36 & 39.699 \\
\hline 9.999 & 90.405 & 39.6 & 9.999 & 90.405 & 39.645 & 9.999 & 90.405 & 39.699 \\
\hline 10.039 & 90.405 & 39.6 & 9.999 & 90.405 & 39.645 & 9.999 & 90.405 & 39.699 \\
\hline 9.978 & 90.405 & 39.6 & 9.999 & 90.405 & 39.645 & 10.019 & 90.405 & 39.699 \\
\hline 9.978 & 90.405 & 39.6 & 9.978 & 90.405 & 39.645 & 10.019 & 90.405 & 39.69 \\
\hline 10.035 & 90.405 & 39.6 & 9.999 & 90.405 & 39.645 & 9.974 & 90.405 & 39.69 \\
\hline 10.015 & 90.405 & 39.6 & 10.019 & 90.405 & 39.645 & 10.015 & 90.405 & 39.69 \\
\hline 9.974 & 90.405 & 39.6 & 10.019 & 90.405 & 39.645 & 10.015 & 90.405 & 39.69 \\
\hline 10.015 & 90.405 & 39.6 & 10.019 & 90.405 & 39.645 & 10.035 & 90.405 & 39.69 \\
\hline 9.954 & 90.405 & 39.6 & 9.999 & 90.405 & 39.645 & 9.974 & 90.405 & 39.69 \\
\hline 9.974 & 90.405 & 39.6 & 9.995 & 90.405 & 39.645 & 9.974 & 90.405 & 39.69 \\
\hline 9.978 & 90.45 & 39.6 & 9.999 & 90.45 & 39.645 & 9.999 & 90.45 & 39.69 \\
\hline 10.019 & 90.45 & 39.6 & 10.019 & 90.45 & 39.645 & 9.978 & 90.45 & 39.69 \\
\hline 9.978 & 90.45 & 39.6 & 9.999 & 90.45 & 39.645 & 9.978 & 90.45 & 39.69 \\
\hline 9.978 & 90.45 & 39.6 & 9.978 & 90.45 & 39.645 & 10.019 & 90.45 & 39.69 \\
\hline 10.019 & 90.45 & 39.6 & 9.978 & 90.45 & 39.645 & 9.978 & 90.45 & 39.69 \\
\hline 10.019 & 90.45 & 39.6 & 9.999 & 90.45 & 39.645 & 10.019 & 90.45 & 39.69 \\
\hline 9.978 & 90.45 & 39.6 & 9.978 & 90.45 & 39.645 & 9.999 & 90.45 & 39.69 \\
\hline 9.999 & 90.45 & 39.6 & 9.978 & 90.45 & 39.645 & 9.978 & 90.45 & 39.69 \\
\hline 9.978 & 90.45 & 39.6 & 9.978 & 90.45 & 39.645 & 9.999 & 90.45 & 39.69 \\
\hline 9.999 & 90.45 & 39.6 & 9.974 & 90.45 & 39.645 & 10.019 & 90.45 & 39.69 \\
\hline 10.019 & 90.495 & 39.6 & 9.999 & 90.495 & 39.645 & 9.978 & 90.495 & 39.69 \\
\hline 10.019 & 90.495 & 39.6 & 9.999 & 90.495 & 39.645 & 10.019 & 90.495 & 39.69 \\
\hline 9.999 & 90.495 & 39.6 & 9.978 & 90.495 & 39.645 & 9.999 & 90.495 & 39.69 \\
\hline 10.019 & 90.495 & 39.6 & 10.019 & 90.495 & 39.645 & 10.019 & 90.495 & 39.69 \\
\hline 9.958 & 90.495 & 39.6 & 10.039 & 90.495 & 39.645 & 10.019 & 90.495 & 39.69 \\
\hline 10.019 & 90.495 & 39.6 & 9.999 & 90.495 & 39.645 & 9.999 & 90.495 & 39.69 \\
\hline 10.019 & 90.495 & 39.6 & 9.978 & 90.495 & 39.645 & 10.019 & 90.495 & 39.69 \\
\hline 10.015 & 90.495 & 39.6 & 9.999 & 90.495 & 39.645 & 10.015 & 90.495 & 39.69 \\
\hline 9.995 & 90.495 & 39.6 & 9.999 & 90.495 & 39.645 & 10.015 & 90.495 & 39.69 \\
\hline 9.995 & 90.495 & 39.6 & 9.978 & 90.495 & 39.645 & 9.995 & 90.495 & 39.69 \\
\hline 9.999 & 90.54 & 39.6 & 10.019 & 90.54 & 39.636 & 9.978 & 90.54 & 39.699 \\
\hline 9.999 & 90.54 & 39.6 & 9.999 & 90.54 & 39.645 & 9.999 & 90.54 & 39.699 \\
\hline 9.978 & 90.54 & 39.6 & 10.019 & 90.54 & 39.645 & 10.019 & 90.54 & 39.699 \\
\hline 9.978 & 90.54 & 39.6 & 9.995 & 90.54 & 39.645 & 9.999 & 90.54 & 39.69 \\
\hline 10.019 & 90.54 & 39.6 & 10.015 & 90.54 & 39.645 & 10.019 & 90.54 & 39.69 \\
\hline 9.999 & 90.54 & 39.6 & 10.015 & 90.54 & 39.645 & 10.019 & 90.54 & 39.69 \\
\hline 9.978 & 90.54 & 39.6 & 9.954 & 90.54 & 39.645 & 9.978 & 90.54 & 39.69 \\
\hline 10.039 & 90.54 & 39.6 & 9.954 & 90.54 & 39.645 & 9.999 & 90.54 & 39.69 \\
\hline 9.999 & 90.54 & 39.6 & 10.015 & 90.54 & 39.645 & 10.019 & 90.54 & 39.69 \\
\hline 10.019 & 90.54 & 39.6 & 9.974 & 90.54 & 39.645 & 10.039 & 90.54 & 39.69 \\
\hline
\end{tabular}


Table B.6b. Data Set 7: Covariance, $0.090^{\circ}$ Incremental Angle.

\begin{tabular}{|c|c|c|c|c|c|c|c|c|}
\hline \multirow{2}{*}{\multicolumn{3}{|c|}{\begin{tabular}{|l} 
Attic, Bldg. 226 \\
Test date: Sept. 3, 2002 \\
Strip 1
\end{tabular}}} & & & & \multicolumn{3}{|c|}{ File: dataset7_covariance_10m_090.xls } \\
\hline & & & \multicolumn{3}{|c|}{ Strip 2} & \multicolumn{3}{|c|}{ Strip 3} \\
\hline Range (m) & $\phi\left({ }^{\circ}\right)$ & $\theta\left({ }^{\circ}\right)$ & Range (m) & $\phi\left({ }^{\circ}\right)$ & $\theta\left({ }^{\circ}\right)$ & Range (m) & $\phi\left({ }^{\circ}\right)$ & $\boldsymbol{\theta}\left({ }^{\circ}\right)$ \\
\hline 10.015 & 88.92 & 39.6 & 10.035 & 88.92 & 39.69 & 10.03 & 88.92 & 39.78 \\
\hline 9.995 & 88.92 & 39.6 & 9.995 & 88.92 & 39.69 & 9.969 & 88.92 & 39.78 \\
\hline 10.015 & 88.92 & 39.6 & 10.035 & 88.92 & 39.69 & 10.01 & 88.92 & 39.78 \\
\hline 9.974 & 88.92 & 39.6 & 10.01 & 88.92 & 39.69 & 9.989 & 88.92 & 39.78 \\
\hline 9.989 & 88.92 & 39.6 & 10.03 & 88.92 & 39.69 & 9.989 & 88.92 & 39.78 \\
\hline 10.01 & 88.92 & 39.6 & 10.03 & 88.92 & 39.69 & 9.969 & 88.92 & 39.78 \\
\hline 10.01 & 88.92 & 39.6 & 10.03 & 88.92 & 39.69 & 9.969 & 88.92 & 39.78 \\
\hline 9.969 & 88.92 & 39.6 & 10.03 & 88.92 & 39.69 & 9.989 & 88.92 & 39.78 \\
\hline 9.969 & 88.92 & 39.6 & 10.01 & 88.92 & 39.69 & 10.01 & 88.92 & 39.78 \\
\hline 10.01 & 88.92 & 39.6 & 10.03 & 88.92 & 39.69 & 9.969 & 88.92 & 39.78 \\
\hline 9.974 & 89.01 & 39.6 & 10.015 & 89.01 & 39.69 & 9.995 & 89.01 & 39.78 \\
\hline 10.035 & 89.01 & 39.6 & 10.015 & 89.01 & 39.69 & 10.015 & 89.01 & 39.78 \\
\hline 10.035 & 89.01 & 39.6 & 9.974 & 89.01 & 39.69 & 10.035 & 89.01 & 39.78 \\
\hline 9.974 & 89.01 & 39.6 & 10.015 & 89.01 & 39.69 & 9.974 & 89.01 & 39.78 \\
\hline 9.995 & 89.01 & 39.6 & 10.015 & 89.01 & 39.69 & 10.015 & 89.01 & 39.78 \\
\hline 9.974 & 89.01 & 39.6 & 10.015 & 89.01 & 39.69 & 10.035 & 89.01 & 39.78 \\
\hline 9.974 & 89.01 & 39.6 & 10.01 & 89.01 & 39.69 & 10.015 & 89.01 & 39.78 \\
\hline 9.989 & 89.01 & 39.6 & 10.01 & 89.01 & 39.69 & 10.01 & 89.01 & 39.78 \\
\hline 10.01 & 89.01 & 39.6 & 10.01 & 89.01 & 39.69 & 9.989 & 89.01 & 39.78 \\
\hline 10.01 & 89.01 & 39.6 & 9.989 & 89.01 & 39.69 & 10.01 & 89.01 & 39.78 \\
\hline 9.974 & 89.1 & 39.6 & 9.954 & 89.1 & 39.69 & 10.015 & 89.1 & 39.78 \\
\hline 10.015 & 89.1 & 39.6 & 9.989 & 89.1 & 39.69 & 10.015 & 89.1 & 39.78 \\
\hline 9.974 & 89.1 & 39.6 & 9.969 & 89.1 & 39.69 & 10.035 & 89.1 & 39.78 \\
\hline 9.995 & 89.1 & 39.6 & 10.01 & 89.1 & 39.69 & 10.035 & 89.1 & 39.78 \\
\hline 9.995 & 89.1 & 39.6 & 10.01 & 89.1 & 39.69 & 10.015 & 89.1 & 39.78 \\
\hline 10.015 & 89.1 & 39.6 & 9.969 & 89.1 & 39.69 & 10.035 & 89.1 & 39.78 \\
\hline 10.015 & 89.1 & 39.6 & 9.969 & 89.1 & 39.69 & 10.015 & 89.1 & 39.78 \\
\hline 10.01 & 89.1 & 39.6 & 10.01 & 89.1 & 39.69 & 9.995 & 89.1 & 39.78 \\
\hline 10.01 & 89.1 & 39.6 & 9.969 & 89.1 & 39.69 & 9.995 & 89.1 & 39.78 \\
\hline 10.01 & 89.1 & 39.6 & 10.01 & 89.1 & 39.69 & 10.03 & 89.1 & 39.78 \\
\hline 10.015 & 89.19 & 39.6 & 10.015 & 89.19 & 39.69 & 9.995 & 89.19 & 39.78 \\
\hline 9.974 & 89.19 & 39.6 & 9.995 & 89.19 & 39.69 & 9.995 & 89.19 & 39.78 \\
\hline 10.015 & 89.19 & 39.6 & 9.974 & 89.19 & 39.69 & 10.015 & 89.19 & 39.78 \\
\hline 9.974 & 89.19 & 39.6 & 9.995 & 89.19 & 39.69 & 10.015 & 89.19 & 39.78 \\
\hline 10.015 & 89.19 & 39.6 & 10.015 & 89.19 & 39.69 & 10.015 & 89.19 & 39.78 \\
\hline 10.015 & 89.19 & 39.6 & 10.015 & 89.19 & 39.69 & 10.035 & 89.19 & 39.78 \\
\hline 10.015 & 89.19 & 39.6 & 10.01 & 89.19 & 39.69 & 10.015 & 89.19 & 39.78 \\
\hline 9.974 & 89.19 & 39.6 & 9.969 & 89.19 & 39.69 & 10.01 & 89.19 & 39.78 \\
\hline 9.969 & 89.19 & 39.6 & 10.01 & 89.19 & 39.69 & 9.989 & 89.19 & 39.78 \\
\hline 9.969 & 89.19 & 39.6 & 9.989 & 89.19 & 39.69 & 10.01 & 89.19 & 39.78 \\
\hline 9.995 & 89.28 & 39.6 & 10.035 & 89.28 & 39.69 & 10.015 & 89.28 & 39.78 \\
\hline 10.015 & 89.28 & 39.6 & 10.015 & 89.28 & 39.69 & 10.015 & 89.28 & 39.78 \\
\hline 10.015 & 89.28 & 39.6 & 10.015 & 89.28 & 39.69 & 9.995 & 89.28 & 39.78 \\
\hline 10.035 & 89.28 & 39.6 & 10.015 & 89.28 & 39.69 & 9.995 & 89.28 & 39.78 \\
\hline 10.015 & 89.28 & 39.6 & 10.015 & 89.28 & 39.69 & 10.015 & 89.28 & 39.78 \\
\hline 10.015 & 89.28 & 39.6 & 10.015 & 89.28 & 39.69 & 10.015 & 89.28 & 39.78 \\
\hline 9.995 & 89.28 & 39.6 & 9.995 & 89.28 & 39.69 & 10.035 & 89.28 & 39.78 \\
\hline 10.015 & 89.28 & 39.6 & 10.015 & 89.28 & 39.69 & 9.989 & 89.28 & 39.78 \\
\hline 10.01 & 89.28 & 39.6 & 10.01 & 89.28 & 39.69 & 9.995 & 89.28 & 39.78 \\
\hline
\end{tabular}


Table B.6b. Data Set 7: Covariance, $0.090^{\circ}$ Incremental Angle.

\begin{tabular}{|c|c|c|c|c|c|c|c|c|}
\hline \multirow{2}{*}{\multicolumn{3}{|c|}{$\begin{array}{l}\text { Attic, Bldg. } 226 \\
\text { Test date: Sept. 3, } 2002 \\
\text { Strip } 1 \\
\end{array}$}} & & & & \multicolumn{3}{|c|}{ File: dataset7_covariance_10m_090.xls } \\
\hline & & & \multicolumn{3}{|c|}{ Strip 2} & \multicolumn{3}{|c|}{ Strip 3} \\
\hline Range (m) & $\phi\left({ }^{\circ}\right)$ & $\theta\left({ }^{\circ}\right)$ & Range (m) & $\phi\left({ }^{0}\right)$ & $\theta\left({ }^{\circ}\right)$ & Range (m) & $\phi\left({ }^{\circ}\right)$ & $\theta\left({ }^{\circ}\right)$ \\
\hline 9.969 & 89.28 & 39.6 & 10.01 & 89.28 & 39.69 & 9.995 & 89.28 & 39.78 \\
\hline 9.995 & 89.37 & 39.6 & 9.974 & 89.37 & 39.69 & 10.015 & 89.37 & 39.78 \\
\hline 9.995 & 89.37 & 39.6 & 10.015 & 89.37 & 39.69 & 9.974 & 89.37 & 39.78 \\
\hline 10.015 & 89.37 & 39.6 & 10.015 & 89.37 & 39.69 & 9.974 & 89.37 & 39.78 \\
\hline 10.055 & 89.37 & 39.6 & 10.015 & 89.37 & 39.69 & 9.974 & 89.37 & 39.78 \\
\hline 10.035 & 89.37 & 39.6 & 10.01 & 89.37 & 39.69 & 10.015 & 89.37 & 39.78 \\
\hline 10.035 & 89.37 & 39.6 & 9.969 & 89.37 & 39.69 & 10.015 & 89.37 & 39.78 \\
\hline 9.974 & 89.37 & 39.6 & 9.969 & 89.37 & 39.69 & 10.035 & 89.37 & 39.78 \\
\hline 10.035 & 89.37 & 39.6 & 10.01 & 89.37 & 39.69 & 10.015 & 89.37 & 39.78 \\
\hline 10.015 & 89.37 & 39.6 & 9.989 & 89.37 & 39.69 & 9.995 & 89.37 & 39.78 \\
\hline 9.989 & 89.37 & 39.6 & 10.01 & 89.37 & 39.69 & 10.01 & 89.37 & 39.78 \\
\hline 10.015 & 89.46 & 39.6 & 10.035 & 89.46 & 39.69 & 10.015 & 89.46 & 39.78 \\
\hline 10.015 & 89.46 & 39.6 & 9.954 & 89.46 & 39.69 & 10.015 & 89.46 & 39.78 \\
\hline 9.954 & 89.46 & 39.6 & 10.035 & 89.46 & 39.69 & 9.974 & 89.46 & 39.78 \\
\hline 10.015 & 89.46 & 39.6 & 10.015 & 89.46 & 39.69 & 9.974 & 89.46 & 39.78 \\
\hline 10.035 & 89.46 & 39.6 & 9.989 & 89.46 & 39.69 & 10.035 & 89.46 & 39.78 \\
\hline 10.015 & 89.46 & 39.6 & 9.989 & 89.46 & 39.69 & 10.015 & 89.46 & 39.78 \\
\hline 10.015 & 89.46 & 39.6 & 10.03 & 89.46 & 39.69 & 10.015 & 89.46 & 39.78 \\
\hline 9.974 & 89.46 & 39.6 & 9.989 & 89.46 & 39.69 & 10.015 & 89.46 & 39.78 \\
\hline 9.995 & 89.46 & 39.6 & 10.01 & 89.46 & 39.69 & 9.995 & 89.46 & 39.78 \\
\hline 10.055 & 89.46 & 39.6 & 9.989 & 89.46 & 39.69 & 9.995 & 89.46 & 39.78 \\
\hline 9.995 & 89.55 & 39.6 & 10.035 & 89.55 & 39.69 & 10.015 & 89.55 & 39.78 \\
\hline 10.015 & 89.55 & 39.6 & 10.015 & 89.55 & 39.69 & 10.035 & 89.55 & 39.78 \\
\hline 10.035 & 89.55 & 39.6 & 9.974 & 89.55 & 39.69 & 10.035 & 89.55 & 39.78 \\
\hline 9.974 & 89.55 & 39.6 & 9.995 & 89.55 & 39.69 & 10.015 & 89.55 & 39.78 \\
\hline 10.015 & 89.55 & 39.6 & 9.995 & 89.55 & 39.69 & 10.015 & 89.55 & 39.78 \\
\hline 10.015 & 89.55 & 39.6 & 9.974 & 89.55 & 39.69 & 9.974 & 89.55 & 39.78 \\
\hline 10.015 & 89.55 & 39.6 & 10.01 & 89.55 & 39.69 & 9.974 & 89.55 & 39.78 \\
\hline 10.015 & 89.55 & 39.6 & 10.01 & 89.55 & 39.69 & 10.035 & 89.55 & 39.78 \\
\hline 10.035 & 89.55 & 39.6 & 9.949 & 89.55 & 39.69 & 10.015 & 89.55 & 39.78 \\
\hline 10.01 & 89.55 & 39.6 & 10.03 & 89.55 & 39.69 & 9.974 & 89.55 & 39.78 \\
\hline 10.015 & 89.64 & 39.6 & 10.055 & 89.64 & 39.69 & 10.015 & 89.64 & 39.78 \\
\hline 10.035 & 89.64 & 39.6 & 10.015 & 89.64 & 39.69 & 10.015 & 89.64 & 39.78 \\
\hline 10.015 & 89.64 & 39.6 & 9.995 & 89.64 & 39.69 & 10.015 & 89.64 & 39.78 \\
\hline 9.974 & 89.64 & 39.6 & 10.015 & 89.64 & 39.69 & 10.015 & 89.64 & 39.78 \\
\hline 9.974 & 89.64 & 39.6 & 9.974 & 89.64 & 39.69 & 9.974 & 89.64 & 39.78 \\
\hline 9.974 & 89.64 & 39.6 & 9.995 & 89.64 & 39.69 & 10.015 & 89.64 & 39.78 \\
\hline 9.974 & 89.64 & 39.6 & 9.974 & 89.64 & 39.69 & 9.974 & 89.64 & 39.78 \\
\hline 10.01 & 89.64 & 39.6 & 10.015 & 89.64 & 39.69 & 10.03 & 89.64 & 39.78 \\
\hline 10.01 & 89.64 & 39.6 & 10.015 & 89.64 & 39.69 & 10.03 & 89.64 & 39.78 \\
\hline 10.01 & 89.64 & 39.6 & 9.969 & 89.64 & 39.69 & 9.969 & 89.64 & 39.78 \\
\hline 10.035 & 89.73 & 39.6 & 10.015 & 89.73 & 39.69 & 10.015 & 89.73 & 39.78 \\
\hline 10.015 & 89.73 & 39.6 & 10.015 & 89.73 & 39.69 & 9.995 & 89.73 & 39.78 \\
\hline 10.015 & 89.73 & 39.6 & 10.035 & 89.73 & 39.69 & 10.015 & 89.73 & 39.78 \\
\hline 9.989 & 89.73 & 39.6 & 10.015 & 89.73 & 39.69 & 10.015 & 89.73 & 39.78 \\
\hline 9.974 & 89.73 & 39.6 & 9.995 & 89.73 & 39.69 & 9.989 & 89.73 & 39.78 \\
\hline 10.01 & 89.73 & 39.6 & 9.974 & 89.73 & 39.69 & 9.995 & 89.73 & 39.78 \\
\hline 10.01 & 89.73 & 39.6 & 9.974 & 89.73 & 39.69 & 9.969 & 89.73 & 39.78 \\
\hline 10.01 & 89.73 & 39.6 & 10.01 & 89.73 & 39.69 & 9.969 & 89.73 & 39.78 \\
\hline 10.01 & 89.73 & 39.6 & 10.03 & 89.73 & 39.69 & 10.03 & 89.73 & 39.78 \\
\hline
\end{tabular}


Table B.6b. Data Set 7: Covariance, 0.090 Incremental Angle.

\begin{tabular}{|c|c|c|c|c|c|c|c|c|}
\hline \multirow{2}{*}{\multicolumn{3}{|c|}{$\begin{array}{l}\text { Attic, Bldg. } 226 \\
\text { Test date: Sept. 3, } 2002 \\
\text { Strip } 1 \\
\end{array}$}} & & & & \multicolumn{3}{|c|}{ File: dataset7_covariance_10m_090.xls } \\
\hline & & & \multicolumn{3}{|c|}{ Strip 2} & \multicolumn{3}{|c|}{ Strip 3} \\
\hline Range (m) & $\phi\left({ }^{\circ}\right)$ & $\theta\left({ }^{\circ}\right)$ & Range (m) & $\phi\left({ }^{0}\right)$ & $\theta\left({ }^{\circ}\right)$ & Range (m) & $\phi\left({ }^{\circ}\right)$ & $\theta\left({ }^{\circ}\right)$ \\
\hline 10.01 & 89.73 & 39.6 & 10.01 & 89.73 & 39.69 & 9.969 & 89.73 & 39.78 \\
\hline 10.015 & 89.82 & 39.6 & 9.974 & 89.82 & 39.69 & 10.015 & 89.82 & 39.78 \\
\hline 10.015 & 89.82 & 39.6 & 9.954 & 89.82 & 39.69 & 9.995 & 89.82 & 39.78 \\
\hline 10.015 & 89.82 & 39.6 & 10.015 & 89.82 & 39.69 & 10.015 & 89.82 & 39.78 \\
\hline 10.015 & 89.82 & 39.6 & 9.974 & 89.82 & 39.69 & 10.015 & 89.82 & 39.78 \\
\hline 9.974 & 89.82 & 39.6 & 10.015 & 89.82 & 39.69 & 10.015 & 89.82 & 39.78 \\
\hline 9.989 & 89.82 & 39.6 & 9.995 & 89.82 & 39.69 & 9.969 & 89.82 & 39.78 \\
\hline 9.969 & 89.82 & 39.6 & 10.01 & 89.82 & 39.69 & 9.969 & 89.82 & 39.78 \\
\hline 10.01 & 89.82 & 39.6 & 9.969 & 89.82 & 39.69 & 10.01 & 89.82 & 39.78 \\
\hline 10.01 & 89.82 & 39.6 & 10.01 & 89.82 & 39.69 & 10.01 & 89.82 & 39.78 \\
\hline 10.01 & 89.82 & 39.6 & 9.989 & 89.82 & 39.69 & 10.01 & 89.82 & 39.78 \\
\hline 9.995 & 89.91 & 39.6 & 9.995 & 89.91 & 39.69 & 9.954 & 89.91 & 39.78 \\
\hline 9.974 & 89.91 & 39.6 & 9.995 & 89.91 & 39.69 & 10.015 & 89.91 & 39.78 \\
\hline 9.974 & 89.91 & 39.6 & 9.995 & 89.91 & 39.69 & 9.989 & 89.91 & 39.78 \\
\hline 10.015 & 89.91 & 39.6 & 10.035 & 89.91 & 39.69 & 10.03 & 89.91 & 39.78 \\
\hline 9.989 & 89.91 & 39.6 & 9.949 & 89.91 & 39.69 & 10.03 & 89.91 & 39.78 \\
\hline 10.01 & 89.91 & 39.6 & 9.989 & 89.91 & 39.69 & 9.974 & 89.91 & 39.78 \\
\hline 9.989 & 89.91 & 39.6 & 9.969 & 89.91 & 39.69 & 9.969 & 89.91 & 39.78 \\
\hline 10.01 & 89.91 & 39.6 & 10.01 & 89.91 & 39.69 & 10.01 & 89.91 & 39.78 \\
\hline 10.01 & 89.91 & 39.6 & 9.989 & 89.91 & 39.69 & 10.03 & 89.91 & 39.78 \\
\hline 10.01 & 89.91 & 39.6 & 9.969 & 89.91 & 39.69 & 10.03 & 89.91 & 39.78 \\
\hline 9.995 & 90 & 39.6 & 9.974 & 90 & 39.69 & 10.015 & 90 & 39.78 \\
\hline 10.035 & 90 & 39.6 & 9.969 & 90 & 39.69 & 10.015 & 90 & 39.78 \\
\hline 9.995 & 90 & 39.6 & 9.974 & 90 & 39.69 & 10.015 & 90 & 39.78 \\
\hline 10.01 & 90 & 39.6 & 10.01 & 90 & 39.69 & 10.01 & 90 & 39.78 \\
\hline 9.989 & 90 & 39.6 & 9.989 & 90 & 39.69 & 10.015 & 90 & 39.78 \\
\hline 9.989 & 90 & 39.6 & 9.989 & 90 & 39.69 & 10.01 & 90 & 39.78 \\
\hline 10.01 & 90 & 39.6 & 9.969 & 90 & 39.69 & 9.989 & 90 & 39.78 \\
\hline 10.01 & 90 & 39.6 & 10.01 & 90 & 39.69 & 9.989 & 90 & 39.78 \\
\hline 10.01 & 90 & 39.6 & 9.969 & 90 & 39.69 & 10.01 & 90 & 39.78 \\
\hline 10.03 & 90 & 39.6 & 10.03 & 90 & 39.69 & 9.969 & 90 & 39.78 \\
\hline 10.015 & 90.09 & 39.6 & 9.995 & 90.09 & 39.69 & 10.015 & 90.09 & 39.78 \\
\hline 9.995 & 90.09 & 39.6 & 10.015 & 90.09 & 39.69 & 10.015 & 90.09 & 39.78 \\
\hline 9.954 & 90.09 & 39.6 & 10.015 & 90.09 & 39.69 & 9.974 & 90.09 & 39.78 \\
\hline 10.015 & 90.09 & 39.6 & 10.015 & 90.09 & 39.69 & 9.969 & 90.09 & 39.78 \\
\hline 10.01 & 90.09 & 39.6 & 9.989 & 90.09 & 39.69 & 10.01 & 90.09 & 39.78 \\
\hline 10.03 & 90.09 & 39.6 & 9.989 & 90.09 & 39.69 & 10.01 & 90.09 & 39.78 \\
\hline 10.01 & 90.09 & 39.6 & 10.01 & 90.09 & 39.69 & 10.01 & 90.09 & 39.78 \\
\hline 9.969 & 90.09 & 39.6 & 9.949 & 90.09 & 39.69 & 10.01 & 90.09 & 39.78 \\
\hline 9.969 & 90.09 & 39.6 & 9.969 & 90.09 & 39.69 & 10.01 & 90.09 & 39.78 \\
\hline 9.989 & 90.09 & 39.6 & 10.01 & 90.09 & 39.69 & 10.01 & 90.09 & 39.78 \\
\hline 10.015 & 90.18 & 39.6 & 10.015 & 90.18 & 39.69 & 9.995 & 90.18 & 39.78 \\
\hline 9.974 & 90.18 & 39.6 & 10.015 & 90.18 & 39.69 & 9.995 & 90.18 & 39.78 \\
\hline 9.995 & 90.18 & 39.6 & 10.015 & 90.18 & 39.69 & 10.015 & 90.18 & 39.78 \\
\hline 9.974 & 90.18 & 39.6 & 10.015 & 90.18 & 39.69 & 10.015 & 90.18 & 39.78 \\
\hline 9.969 & 90.18 & 39.6 & 9.969 & 90.18 & 39.69 & 9.989 & 90.18 & 39.78 \\
\hline 9.969 & 90.18 & 39.6 & 9.969 & 90.18 & 39.69 & 9.969 & 90.18 & 39.78 \\
\hline 9.989 & 90.18 & 39.6 & 10.01 & 90.18 & 39.69 & 9.969 & 90.18 & 39.78 \\
\hline 10.01 & 90.18 & 39.6 & 10.01 & 90.18 & 39.69 & 9.969 & 90.18 & 39.78 \\
\hline 10.03 & 90.18 & 39.6 & 10.05 & 90.18 & 39.69 & 9.969 & 90.18 & 39.78 \\
\hline
\end{tabular}


Table B.6b. Data Set 7: Covariance, $0.090^{\circ}$ Incremental Angle.

\begin{tabular}{|c|c|c|c|c|c|c|c|c|}
\hline \multirow{2}{*}{\multicolumn{3}{|c|}{$\begin{array}{l}\text { Attic, Bldg. } 226 \\
\text { Test date: Sept. 3, } 2002 \\
\text { Strip } 1 \\
\end{array}$}} & & & & \multicolumn{3}{|c|}{ File: dataset7_covariance_10m_090.xls } \\
\hline & & & \multicolumn{3}{|c|}{ Strip 2} & \multicolumn{3}{|c|}{ Strip 3} \\
\hline Range (m) & $\phi\left({ }^{\circ}\right)$ & $\theta\left({ }^{\circ}\right)$ & Range (m) & $\phi\left({ }^{0}\right)$ & $\theta\left({ }^{\circ}\right)$ & Range (m) & $\phi\left({ }^{\circ}\right)$ & $\theta\left({ }^{\circ}\right)$ \\
\hline 10.01 & 90.18 & 39.6 & 10.03 & 90.18 & 39.69 & 9.989 & 90.18 & 39.78 \\
\hline 10.015 & 90.27 & 39.6 & 9.954 & 90.27 & 39.69 & 9.974 & 90.27 & 39.78 \\
\hline 10.015 & 90.27 & 39.6 & 9.995 & 90.27 & 39.69 & 10.035 & 90.27 & 39.78 \\
\hline 10.035 & 90.27 & 39.6 & 9.954 & 90.27 & 39.69 & 9.974 & 90.27 & 39.78 \\
\hline 9.995 & 90.27 & 39.6 & 9.995 & 90.27 & 39.69 & 9.995 & 90.27 & 39.78 \\
\hline 9.995 & 90.27 & 39.6 & 10.015 & 90.27 & 39.69 & 10.015 & 90.27 & 39.78 \\
\hline 10.015 & 90.27 & 39.6 & 9.995 & 90.27 & 39.69 & 9.995 & 90.27 & 39.78 \\
\hline 10.015 & 90.27 & 39.6 & 10.015 & 90.27 & 39.69 & 10.015 & 90.27 & 39.78 \\
\hline 10.03 & 90.27 & 39.6 & 9.974 & 90.27 & 39.69 & 10.015 & 90.27 & 39.78 \\
\hline 9.969 & 90.27 & 39.6 & 9.969 & 90.27 & 39.69 & 9.989 & 90.27 & 39.78 \\
\hline 9.969 & 90.27 & 39.6 & 10.03 & 90.27 & 39.69 & 10.01 & 90.27 & 39.78 \\
\hline 10.015 & 90.36 & 39.6 & 9.954 & 90.36 & 39.69 & 10.015 & 90.36 & 39.78 \\
\hline 10.015 & 90.36 & 39.6 & 10.035 & 90.36 & 39.69 & 10.015 & 90.36 & 39.78 \\
\hline 10.015 & 90.36 & 39.6 & 10.015 & 90.36 & 39.69 & 9.974 & 90.36 & 39.78 \\
\hline 9.995 & 90.36 & 39.6 & 10.015 & 90.36 & 39.69 & 10.015 & 90.36 & 39.78 \\
\hline 10.015 & 90.36 & 39.6 & 9.974 & 90.36 & 39.69 & 10.015 & 90.36 & 39.78 \\
\hline 10.015 & 90.36 & 39.6 & 9.974 & 90.36 & 39.69 & 10.015 & 90.36 & 39.78 \\
\hline 10.035 & 90.36 & 39.6 & 9.989 & 90.36 & 39.69 & 10.015 & 90.36 & 39.78 \\
\hline 9.974 & 90.36 & 39.6 & 10.01 & 90.36 & 39.69 & 10.015 & 90.36 & 39.78 \\
\hline 10.01 & 90.36 & 39.6 & 9.969 & 90.36 & 39.69 & 9.969 & 90.36 & 39.78 \\
\hline 10.01 & 90.36 & 39.6 & 9.969 & 90.36 & 39.69 & 9.969 & 90.36 & 39.78 \\
\hline 9.974 & 90.45 & 39.6 & 10.015 & 90.45 & 39.69 & 9.974 & 90.45 & 39.78 \\
\hline 10.015 & 90.45 & 39.6 & 10.015 & 90.45 & 39.69 & 9.974 & 90.45 & 39.78 \\
\hline 10.015 & 90.45 & 39.6 & 10.015 & 90.45 & 39.69 & 9.974 & 90.45 & 39.78 \\
\hline 10.015 & 90.45 & 39.6 & 9.974 & 90.45 & 39.69 & 9.954 & 90.45 & 39.78 \\
\hline 10.015 & 90.45 & 39.6 & 9.995 & 90.45 & 39.69 & 10.015 & 90.45 & 39.78 \\
\hline 10.015 & 90.45 & 39.6 & 10.035 & 90.45 & 39.69 & 10.015 & 90.45 & 39.78 \\
\hline 10.015 & 90.45 & 39.6 & 9.974 & 90.45 & 39.69 & 10.01 & 90.45 & 39.78 \\
\hline 10.01 & 90.45 & 39.6 & 10.015 & 90.45 & 39.69 & 10.01 & 90.45 & 39.78 \\
\hline 9.969 & 90.45 & 39.6 & 9.995 & 90.45 & 39.69 & 9.969 & 90.45 & 39.78 \\
\hline 10.01 & 90.45 & 39.6 & 9.969 & 90.45 & 39.69 & 9.989 & 90.45 & 39.78 \\
\hline 10.015 & 90.54 & 39.6 & 10.015 & 90.54 & 39.69 & 10.015 & 90.54 & 39.78 \\
\hline 10.015 & 90.54 & 39.6 & 10.015 & 90.54 & 39.69 & 9.995 & 90.54 & 39.78 \\
\hline 9.995 & 90.54 & 39.6 & 10.015 & 90.54 & 39.69 & 9.995 & 90.54 & 39.78 \\
\hline 9.974 & 90.54 & 39.6 & 9.974 & 90.54 & 39.69 & 9.995 & 90.54 & 39.78 \\
\hline 10.015 & 90.54 & 39.6 & 10.035 & 90.54 & 39.69 & 10.015 & 90.54 & 39.78 \\
\hline 10.035 & 90.54 & 39.6 & 10.035 & 90.54 & 39.69 & 10.015 & 90.54 & 39.78 \\
\hline 10.015 & 90.54 & 39.6 & 9.974 & 90.54 & 39.69 & 10.015 & 90.54 & 39.78 \\
\hline 9.995 & 90.54 & 39.6 & 10.055 & 90.54 & 39.69 & 10.01 & 90.54 & 39.78 \\
\hline 9.969 & 90.54 & 39.6 & 9.974 & 90.54 & 39.69 & 9.969 & 90.54 & 39.78 \\
\hline 9.969 & 90.54 & 39.6 & 10.015 & 90.54 & 39.69 & 10.03 & 90.54 & 39.78 \\
\hline 10.015 & 90.63 & 39.6 & 10.015 & 90.63 & 39.69 & 10.015 & 90.63 & 39.78 \\
\hline 9.974 & 90.63 & 39.6 & 9.995 & 90.63 & 39.69 & 10.015 & 90.63 & 39.78 \\
\hline 10.015 & 90.63 & 39.6 & 10.015 & 90.63 & 39.69 & 10.015 & 90.63 & 39.78 \\
\hline 9.974 & 90.63 & 39.6 & 9.995 & 90.63 & 39.69 & 9.995 & 90.63 & 39.78 \\
\hline 9.974 & 90.63 & 39.6 & 10.015 & 90.63 & 39.69 & 9.969 & 90.63 & 39.78 \\
\hline 9.969 & 90.63 & 39.6 & 9.995 & 90.63 & 39.69 & 10.03 & 90.63 & 39.78 \\
\hline 9.969 & 90.63 & 39.6 & 10.015 & 90.63 & 39.69 & 10.01 & 90.63 & 39.78 \\
\hline 9.989 & 90.63 & 39.6 & 9.974 & 90.63 & 39.69 & 10.03 & 90.63 & 39.78 \\
\hline 10.01 & 90.63 & 39.6 & 9.995 & 90.63 & 39.69 & 10.03 & 90.63 & 39.78 \\
\hline
\end{tabular}


Table B.6b. Data Set 7: Covariance, $0.090^{\circ}$ Incremental Angle.

\begin{tabular}{|c|c|c|c|c|c|c|c|c|}
\hline \multirow{2}{*}{\multicolumn{3}{|c|}{$\begin{array}{l}\text { Attic, Bldg. } 226 \\
\text { Test date: Sept. 3, } 2002 \\
\text { Strip } 1 \\
\end{array}$}} & & & & \multicolumn{3}{|c|}{ File: dataset7_covariance_10m_090.xls } \\
\hline & & & \multicolumn{3}{|c|}{ Strip 2} & \multicolumn{3}{|c|}{ Strip 3} \\
\hline Range (m) & $\phi\left({ }^{\circ}\right)$ & $\theta\left({ }^{\circ}\right)$ & Range (m) & $\phi\left({ }^{0}\right)$ & $\theta\left({ }^{\circ}\right)$ & Range (m) & $\phi\left({ }^{\circ}\right)$ & $\theta\left({ }^{\circ}\right)$ \\
\hline 10.03 & 90.63 & 39.6 & 9.995 & 90.63 & 39.69 & 10.01 & 90.63 & 39.78 \\
\hline 10.015 & 90.72 & 39.6 & 9.995 & 90.72 & 39.69 & 9.954 & 90.72 & 39.78 \\
\hline 9.995 & 90.72 & 39.6 & 9.974 & 90.72 & 39.69 & 10.015 & 90.72 & 39.78 \\
\hline 10.035 & 90.72 & 39.6 & 9.974 & 90.72 & 39.69 & 10.015 & 90.72 & 39.78 \\
\hline 10.015 & 90.72 & 39.6 & 9.974 & 90.72 & 39.69 & 10.015 & 90.72 & 39.78 \\
\hline 10.015 & 90.72 & 39.6 & 10.015 & 90.72 & 39.69 & 9.995 & 90.72 & 39.78 \\
\hline 10.015 & 90.72 & 39.6 & 10.035 & 90.72 & 39.69 & 9.974 & 90.72 & 39.78 \\
\hline 9.995 & 90.72 & 39.6 & 9.974 & 90.72 & 39.69 & 9.974 & 90.72 & 39.78 \\
\hline 9.995 & 90.72 & 39.6 & 10.015 & 90.72 & 39.69 & 9.974 & 90.72 & 39.78 \\
\hline 9.974 & 90.72 & 39.6 & 10.035 & 90.72 & 39.69 & 10.035 & 90.72 & 39.78 \\
\hline 10.05 & 90.72 & 39.6 & 10.015 & 90.72 & 39.69 & 9.974 & 90.72 & 39.78 \\
\hline 9.954 & 90.81 & 39.6 & 10.015 & 90.81 & 39.69 & 9.995 & 90.81 & 39.78 \\
\hline 10.015 & 90.81 & 39.6 & 10.015 & 90.81 & 39.69 & 10.015 & 90.81 & 39.78 \\
\hline 9.969 & 90.81 & 39.6 & 10.035 & 90.81 & 39.69 & 10.055 & 90.81 & 39.78 \\
\hline 10.01 & 90.81 & 39.6 & 9.995 & 90.81 & 39.69 & 9.989 & 90.81 & 39.78 \\
\hline 10.01 & 90.81 & 39.6 & 9.995 & 90.81 & 39.69 & 10.01 & 90.81 & 39.78 \\
\hline 10.01 & 90.81 & 39.6 & 9.995 & 90.81 & 39.69 & 10.01 & 90.81 & 39.78 \\
\hline 10.01 & 90.81 & 39.6 & 10.015 & 90.81 & 39.69 & 9.989 & 90.81 & 39.78 \\
\hline 10.01 & 90.81 & 39.6 & 9.974 & 90.81 & 39.69 & 9.969 & 90.81 & 39.78 \\
\hline 10.03 & 90.81 & 39.6 & 9.954 & 90.81 & 39.69 & 9.969 & 90.81 & 39.78 \\
\hline 9.969 & 90.81 & 39.6 & 9.989 & 90.81 & 39.69 & 9.969 & 90.81 & 39.78 \\
\hline 10.015 & 90.9 & 39.6 & 10.015 & 90.9 & 39.69 & 9.995 & 90.9 & 39.78 \\
\hline 10.035 & 90.9 & 39.6 & 9.995 & 90.9 & 39.69 & 10.015 & 90.9 & 39.78 \\
\hline 10.015 & 90.9 & 39.6 & 10.015 & 90.9 & 39.69 & 9.974 & 90.9 & 39.78 \\
\hline 9.995 & 90.9 & 39.6 & 10.035 & 90.9 & 39.69 & 10.055 & 90.9 & 39.78 \\
\hline 9.989 & 90.9 & 39.6 & 9.974 & 90.9 & 39.69 & 10.015 & 90.9 & 39.78 \\
\hline 9.989 & 90.9 & 39.6 & 9.974 & 90.9 & 39.69 & 10.01 & 90.9 & 39.78 \\
\hline 9.989 & 90.9 & 39.6 & 9.974 & 90.9 & 39.69 & 9.989 & 90.9 & 39.78 \\
\hline 10.03 & 90.9 & 39.6 & 10.015 & 90.9 & 39.69 & 10.01 & 90.9 & 39.78 \\
\hline 10.01 & 90.9 & 39.6 & 10.035 & 90.9 & 39.69 & 10.03 & 90.9 & 39.78 \\
\hline 10.01 & 90.9 & 39.6 & 10.015 & 90.9 & 39.69 & 9.989 & 90.9 & 39.78 \\
\hline 10.015 & 90.99 & 39.6 & 10.035 & 90.99 & 39.69 & 9.995 & 90.99 & 39.78 \\
\hline 9.995 & 90.99 & 39.6 & 10.015 & 90.99 & 39.69 & 9.995 & 90.99 & 39.78 \\
\hline 10.01 & 90.99 & 39.6 & 9.974 & 90.99 & 39.69 & 9.974 & 90.99 & 39.78 \\
\hline 10.01 & 90.99 & 39.6 & 9.969 & 90.99 & 39.69 & 9.989 & 90.99 & 39.78 \\
\hline 10.01 & 90.99 & 39.6 & 10.015 & 90.99 & 39.69 & 10.03 & 90.99 & 39.78 \\
\hline 9.989 & 90.99 & 39.6 & 10.015 & 90.99 & 39.69 & 10.01 & 90.99 & 39.78 \\
\hline 9.969 & 90.99 & 39.6 & 9.969 & 90.99 & 39.69 & 10.01 & 90.99 & 39.78 \\
\hline 9.989 & 90.99 & 39.6 & 10.01 & 90.99 & 39.69 & 9.989 & 90.99 & 39.78 \\
\hline 9.969 & 90.99 & 39.6 & 10.01 & 90.99 & 39.69 & 10.01 & 90.99 & 39.78 \\
\hline 9.989 & 90.99 & 39.6 & 10.01 & 90.99 & 39.69 & 10.01 & 90.99 & 39.78 \\
\hline 10.015 & 91.08 & 39.6 & 10.015 & 91.08 & 39.69 & 10.015 & 91.08 & 39.78 \\
\hline 10.015 & 91.08 & 39.6 & 9.995 & 91.08 & 39.69 & 9.974 & 91.08 & 39.78 \\
\hline 10.01 & 91.08 & 39.6 & 9.989 & 91.08 & 39.69 & 10.055 & 91.08 & 39.78 \\
\hline 9.969 & 91.08 & 39.6 & 9.989 & 91.08 & 39.69 & 10.01 & 91.08 & 39.78 \\
\hline 9.989 & 91.08 & 39.6 & 10.01 & 91.08 & 39.69 & 10.03 & 91.08 & 39.78 \\
\hline 9.969 & 91.08 & 39.6 & 10.01 & 91.08 & 39.69 & 10.01 & 91.08 & 39.78 \\
\hline 9.989 & 91.08 & 39.6 & 10.01 & 91.08 & 39.69 & 9.989 & 91.08 & 39.78 \\
\hline 10.01 & 91.08 & 39.6 & 10.01 & 91.08 & 39.69 & 9.989 & 91.08 & 39.78 \\
\hline 10.01 & 91.08 & 39.6 & 10.01 & 91.08 & 39.69 & 10.03 & 91.08 & 39.78 \\
\hline
\end{tabular}


Table B.6b. Data Set 7: Covariance, $0.090^{\circ}$ Incremental Angle.

\begin{tabular}{|c|c|c|c|c|c|c|c|c|}
\hline \multirow{2}{*}{\multicolumn{9}{|c|}{$\begin{array}{l}\text { Attic, Bldg. } 226 \\
\text { Test date: Sept. 3, } 2002\end{array}$}} \\
\hline & & & & & & & & \\
\hline \multicolumn{3}{|c|}{ Strip 1} & \multicolumn{3}{|c|}{ Strip 2} & \multicolumn{3}{|c|}{ Strip 3} \\
\hline Range (m) & $\phi\left({ }^{\circ}\right)$ & $\theta\left({ }^{\circ}\right)$ & Range (m) & $\phi\left({ }^{\circ}\right)$ & $\theta\left({ }^{\circ}\right)$ & Range (m) & $\phi\left(^{\circ}\right)$ & $\theta\left(\left(^{\circ}\right)\right.$ \\
\hline 9.969 & 91.08 & 39.6 & 10.01 & 91.08 & 39.69 & 10.03 & 91.08 & 39.78 \\
\hline
\end{tabular}

Table B.6c. Data Set 7: Covariance, $0.180^{\circ}$ Incremental Angle.

\begin{tabular}{|c|c|c|c|c|c|c|c|c|}
\hline \multirow{2}{*}{\multicolumn{3}{|c|}{\begin{tabular}{|l} 
Attic, Bldg. 226 \\
Test date: Sept. 3, 2002
\end{tabular}}} & & & & \multicolumn{3}{|c|}{ File: dataset7_covariance_10m_180.xls } \\
\hline & & & \multicolumn{3}{|c|}{ Strip 2} & \multicolumn{3}{|c|}{ Strip 3} \\
\hline Range (m) & $\phi\left({ }^{0}\right)$ & $\theta\left({ }^{\circ}\right)$ & Range (m) & $\phi\left({ }^{0}\right)$ & $\theta\left({ }^{\circ}\right)$ & Range (m) & $\phi\left({ }^{0}\right)$ & $\theta\left({ }^{\circ}\right)$ \\
\hline 10.01 & 87.84 & 39.6 & 10.01 & 87.84 & 39.789 & 10.03 & 87.84 & 39.951 \\
\hline 10.01 & 87.84 & 39.6 & 10.01 & 87.84 & 39.789 & 10.03 & 87.84 & 39.951 \\
\hline 10.03 & 87.84 & 39.6 & 10.03 & 87.84 & 39.789 & 10.01 & 87.84 & 39.951 \\
\hline 10.03 & 87.84 & 39.6 & 9.969 & 87.84 & 39.789 & 10.01 & 87.84 & 39.951 \\
\hline 10.03 & 87.84 & 39.6 & 9.969 & 87.84 & 39.789 & 10.03 & 87.84 & 39.951 \\
\hline 9.989 & 87.84 & 39.6 & 10.01 & 87.84 & 39.789 & 10.03 & 87.84 & 39.951 \\
\hline 10.025 & 87.84 & 39.6 & 10.01 & 87.84 & 39.789 & 10.01 & 87.84 & 39.951 \\
\hline 10.005 & 87.84 & 39.6 & 10.01 & 87.84 & 39.789 & 9.989 & 87.84 & 39.951 \\
\hline 10.045 & 87.84 & 39.6 & 10.03 & 87.84 & 39.789 & 9.969 & 87.84 & 39.951 \\
\hline 10.025 & 87.84 & 39.6 & 9.989 & 87.84 & 39.789 & 9.969 & 87.84 & 39.951 \\
\hline 9.969 & 88.02 & 39.6 & 10.01 & 88.02 & 39.78 & 10.03 & 88.02 & 39.96 \\
\hline 10.03 & 88.02 & 39.6 & 10.01 & 88.02 & 39.78 & 10.03 & 88.02 & 39.96 \\
\hline 10.01 & 88.02 & 39.6 & 10.03 & 88.02 & 39.78 & 10.03 & 88.02 & 39.96 \\
\hline 9.989 & 88.02 & 39.6 & 9.989 & 88.02 & 39.78 & 10.03 & 88.02 & 39.96 \\
\hline 10.03 & 88.02 & 39.6 & 9.989 & 88.02 & 39.78 & 10.05 & 88.02 & 39.96 \\
\hline 10.03 & 88.02 & 39.6 & 9.989 & 88.02 & 39.789 & 10.03 & 88.02 & 39.96 \\
\hline 10.025 & 88.02 & 39.6 & 10.03 & 88.02 & 39.789 & 9.989 & 88.02 & 39.96 \\
\hline 9.989 & 88.02 & 39.6 & 10.03 & 88.02 & 39.789 & 10.05 & 88.02 & 39.96 \\
\hline 10.005 & 88.02 & 39.6 & 9.989 & 88.02 & 39.789 & 10.005 & 88.02 & 39.96 \\
\hline 10.025 & 88.02 & 39.6 & 10.03 & 88.02 & 39.789 & 10.005 & 88.02 & 39.96 \\
\hline 10.05 & 88.2 & 39.6 & 10.01 & 88.2 & 39.78 & 10.01 & 88.2 & 39.951 \\
\hline 10.03 & 88.2 & 39.6 & 10.01 & 88.2 & 39.78 & 10.03 & 88.2 & 39.951 \\
\hline 9.989 & 88.2 & 39.6 & 10.01 & 88.2 & 39.78 & 10.03 & 88.2 & 39.96 \\
\hline 10.05 & 88.2 & 39.6 & 10.01 & 88.2 & 39.78 & 10.03 & 88.2 & 39.951 \\
\hline 10.05 & 88.2 & 39.6 & 10.03 & 88.2 & 39.78 & 9.969 & 88.2 & 39.951 \\
\hline 10.03 & 88.2 & 39.6 & 10.03 & 88.2 & 39.789 & 10.01 & 88.2 & 39.951 \\
\hline 10.01 & 88.2 & 39.6 & 9.969 & 88.2 & 39.789 & 10.01 & 88.2 & 39.951 \\
\hline 10.01 & 88.2 & 39.6 & 10.045 & 88.2 & 39.789 & 10.05 & 88.2 & 39.951 \\
\hline 10.01 & 88.2 & 39.6 & 10.025 & 88.2 & 39.789 & 9.984 & 88.2 & 39.951 \\
\hline 10.005 & 88.2 & 39.6 & 9.984 & 88.2 & 39.789 & 10.045 & 88.2 & 39.96 \\
\hline 10.05 & 88.38 & 39.6 & 9.989 & 88.38 & 39.78 & 10.03 & 88.38 & 39.96 \\
\hline 10.01 & 88.38 & 39.6 & 10.05 & 88.38 & 39.78 & 10.01 & 88.38 & 39.96 \\
\hline 10.03 & 88.38 & 39.6 & 10.03 & 88.38 & 39.78 & 10.05 & 88.38 & 39.96 \\
\hline 10.01 & 88.38 & 39.6 & 10.03 & 88.38 & 39.78 & 10.01 & 88.38 & 39.96 \\
\hline 10.01 & 88.38 & 39.6 & 10.03 & 88.38 & 39.78 & 10.03 & 88.38 & 39.96 \\
\hline 9.969 & 88.38 & 39.6 & 10.03 & 88.38 & 39.78 & 9.984 & 88.38 & 39.96 \\
\hline 10.025 & 88.38 & 39.6 & 10.01 & 88.38 & 39.78 & 10.025 & 88.38 & 39.96 \\
\hline 10.005 & 88.38 & 39.6 & 10.03 & 88.38 & 39.78 & 9.984 & 88.38 & 39.96 \\
\hline 10.005 & 88.38 & 39.6 & 10.025 & 88.38 & 39.78 & 9.964 & 88.38 & 39.96 \\
\hline 10.005 & 88.38 & 39.6 & 10.005 & 88.38 & 39.78 & 10.005 & 88.38 & 39.96 \\
\hline 10.01 & 88.56 & 39.6 & 9.969 & 88.56 & 39.78 & 10.05 & 88.56 & 39.96 \\
\hline 9.989 & 88.56 & 39.6 & 9.989 & 88.56 & 39.78 & 10.01 & 88.56 & 39.96 \\
\hline 10.01 & 88.56 & 39.6 & 9.969 & 88.56 & 39.78 & 10.01 & 88.56 & 39.96 \\
\hline 10.03 & 88.56 & 39.6 & 10.01 & 88.56 & 39.78 & 10.01 & 88.56 & 39.96 \\
\hline
\end{tabular}


Table B.6c. Data Set 7: Covariance, $0.180^{\circ}$ Incremental Angle.

\begin{tabular}{|c|c|c|c|c|c|c|c|c|}
\hline \multirow{2}{*}{\multicolumn{3}{|c|}{\begin{tabular}{|l|} 
Attic, Bldg. 226 \\
Test date: Sept. 3, 2002 \\
Strip 1
\end{tabular}}} & & & & \multicolumn{3}{|c|}{ File: dataset7_covariance_10m_180.xls } \\
\hline & & & & Strip & & & Strip & \\
\hline Range (m) & $\phi\left(^{0}\right)$ & $\theta\left({ }^{\circ}\right)$ & Range (m) & $\phi\left({ }^{0}\right)$ & $\theta\left(^{0}\right)$ & Range (m) & $\phi\left({ }^{0}\right)$ & $\theta\left(^{0}\right)$ \\
\hline 9.989 & 88.56 & 39.6 & 10.03 & 88.56 & 39.78 & 10.01 & 88.56 & 39.96 \\
\hline 10.01 & 88.56 & 39.6 & 10.03 & 88.56 & 39.78 & 10.01 & 88.56 & 39.96 \\
\hline 10.03 & 88.56 & 39.6 & 10.01 & 88.56 & 39.78 & 9.989 & 88.56 & 39.96 \\
\hline 10.01 & 88.56 & 39.6 & 10.03 & 88.56 & 39.78 & 10.01 & 88.56 & 39.96 \\
\hline 9.989 & 88.56 & 39.6 & 10.03 & 88.56 & 39.78 & 10.05 & 88.56 & 39.96 \\
\hline 9.964 & 88.56 & 39.6 & 10.01 & 88.56 & 39.78 & 10.025 & 88.56 & 39.96 \\
\hline 10.01 & 88.74 & 39.6 & 9.969 & 88.74 & 39.78 & 9.969 & 88.74 & 39.951 \\
\hline 9.989 & 88.74 & 39.6 & 9.969 & 88.74 & 39.78 & 10.03 & 88.74 & 39.951 \\
\hline 10.03 & 88.74 & 39.6 & 10.01 & 88.74 & 39.78 & 9.989 & 88.74 & 39.96 \\
\hline 10.03 & 88.74 & 39.6 & 9.989 & 88.74 & 39.78 & 9.969 & 88.74 & 39.951 \\
\hline 10.03 & 88.74 & 39.6 & 9.989 & 88.74 & 39.789 & 10.03 & 88.74 & 39.951 \\
\hline 9.989 & 88.74 & 39.6 & 9.989 & 88.74 & 39.789 & 10.03 & 88.74 & 39.951 \\
\hline 10.01 & 88.74 & 39.6 & 10.01 & 88.74 & 39.789 & 10.01 & 88.74 & 39.951 \\
\hline 9.989 & 88.74 & 39.6 & 10.05 & 88.74 & 39.789 & 10.03 & 88.74 & 39.951 \\
\hline 9.969 & 88.74 & 39.6 & 10.01 & 88.74 & 39.789 & 10.03 & 88.74 & 39.96 \\
\hline 10.03 & 88.74 & 39.6 & 9.989 & 88.74 & 39.789 & 10.01 & 88.74 & 39.96 \\
\hline 10.03 & 88.92 & 39.6 & 10.03 & 88.92 & 39.789 & 10.01 & 88.92 & 39.951 \\
\hline 10.03 & 88.92 & 39.6 & 9.969 & 88.92 & 39.789 & 9.989 & 88.92 & 39.951 \\
\hline 10.01 & 88.92 & 39.6 & 10.01 & 88.92 & 39.789 & 10.01 & 88.92 & 39.951 \\
\hline 10.03 & 88.92 & 39.6 & 9.969 & 88.92 & 39.789 & 10.05 & 88.92 & 39.951 \\
\hline 10.01 & 88.92 & 39.6 & 10.01 & 88.92 & 39.789 & 10.05 & 88.92 & 39.951 \\
\hline 10.05 & 88.92 & 39.6 & 9.989 & 88.92 & 39.789 & 9.969 & 88.92 & 39.951 \\
\hline 9.989 & 88.92 & 39.6 & 10.01 & 88.92 & 39.789 & 9.989 & 88.92 & 39.951 \\
\hline 10.01 & 88.92 & 39.6 & 10.01 & 88.92 & 39.789 & 10.03 & 88.92 & 39.951 \\
\hline 10.01 & 88.92 & 39.6 & 10.05 & 88.92 & 39.789 & 10.05 & 88.92 & 39.951 \\
\hline 9.989 & 88.92 & 39.6 & 9.969 & 88.92 & 39.789 & 10.03 & 88.92 & 39.951 \\
\hline 9.989 & 89.1 & 39.6 & 10.01 & 89.1 & 39.789 & 9.969 & 89.1 & 39.96 \\
\hline 10.01 & 89.1 & 39.6 & 10.03 & 89.1 & 39.789 & 9.969 & 89.1 & 39.96 \\
\hline 10.01 & 89.1 & 39.6 & 9.989 & 89.1 & 39.789 & 10.01 & 89.1 & 39.96 \\
\hline 10.03 & 89.1 & 39.6 & 10.01 & 89.1 & 39.789 & 10.01 & 89.1 & 39.96 \\
\hline 10.01 & 89.1 & 39.6 & 10.03 & 89.1 & 39.789 & 10.01 & 89.1 & 39.96 \\
\hline 9.969 & 89.1 & 39.6 & 9.969 & 89.1 & 39.789 & 10.01 & 89.1 & 39.96 \\
\hline 10.03 & 89.1 & 39.6 & 10.01 & 89.1 & 39.789 & 10.01 & 89.1 & 39.96 \\
\hline 10.025 & 89.1 & 39.6 & 10.05 & 89.1 & 39.789 & 9.989 & 89.1 & 39.96 \\
\hline 10.01 & 89.1 & 39.6 & 9.969 & 89.1 & 39.789 & 9.969 & 89.1 & 39.96 \\
\hline 10.03 & 89.1 & 39.6 & 10.03 & 89.1 & 39.789 & 9.969 & 89.1 & 39.96 \\
\hline 10.01 & 89.28 & 39.6 & 9.989 & 89.28 & 39.789 & 10.01 & 89.28 & 39.96 \\
\hline 9.969 & 89.28 & 39.6 & 10.01 & 89.28 & 39.789 & 9.989 & 89.28 & 39.96 \\
\hline 10.03 & 89.28 & 39.6 & 10.01 & 89.28 & 39.789 & 10.03 & 89.28 & 39.96 \\
\hline 10.01 & 89.28 & 39.6 & 9.989 & 89.28 & 39.789 & 10.05 & 89.28 & 39.96 \\
\hline 10.05 & 89.28 & 39.6 & 10.03 & 89.28 & 39.789 & 10.01 & 89.28 & 39.96 \\
\hline 9.969 & 89.28 & 39.6 & 10.03 & 89.28 & 39.789 & 10.03 & 89.28 & 39.96 \\
\hline 10.01 & 89.28 & 39.6 & 10.01 & 89.28 & 39.789 & 10.01 & 89.28 & 39.96 \\
\hline 10.03 & 89.28 & 39.6 & 10.01 & 89.28 & 39.789 & 9.989 & 89.28 & 39.96 \\
\hline 10.05 & 89.28 & 39.6 & 10.03 & 89.28 & 39.789 & 10.01 & 89.28 & 39.96 \\
\hline 9.964 & 89.28 & 39.6 & 10.01 & 89.28 & 39.789 & 9.984 & 89.28 & 39.96 \\
\hline 10.01 & 89.46 & 39.6 & 9.969 & 89.46 & 39.789 & 9.969 & 89.46 & 39.96 \\
\hline 10.01 & 89.46 & 39.6 & 10.01 & 89.46 & 39.789 & 10.03 & 89.46 & 39.96 \\
\hline 10.01 & 89.46 & 39.6 & 10.01 & 89.46 & 39.789 & 10.01 & 89.46 & 39.96 \\
\hline 10.01 & 89.46 & 39.6 & 10.03 & 89.46 & 39.789 & 10.01 & 89.46 & 39.96 \\
\hline 10.01 & 89.46 & 39.6 & 10.01 & 89.46 & 39.789 & 9.969 & 89.46 & 39.96 \\
\hline 9.969 & 89.46 & 39.6 & 10.01 & 89.46 & 39.789 & 9.989 & 89.46 & 39.96 \\
\hline 10.01 & 89.46 & 39.6 & 10.03 & 89.46 & 39.789 & 10.005 & 89.46 & 39.96 \\
\hline 10.025 & 89.46 & 39.6 & 10.01 & 89.46 & 39.789 & 10.025 & 89.46 & 39.96 \\
\hline
\end{tabular}


Table B.6c. Data Set 7: Covariance, $0.180^{\circ}$ Incremental Angle.

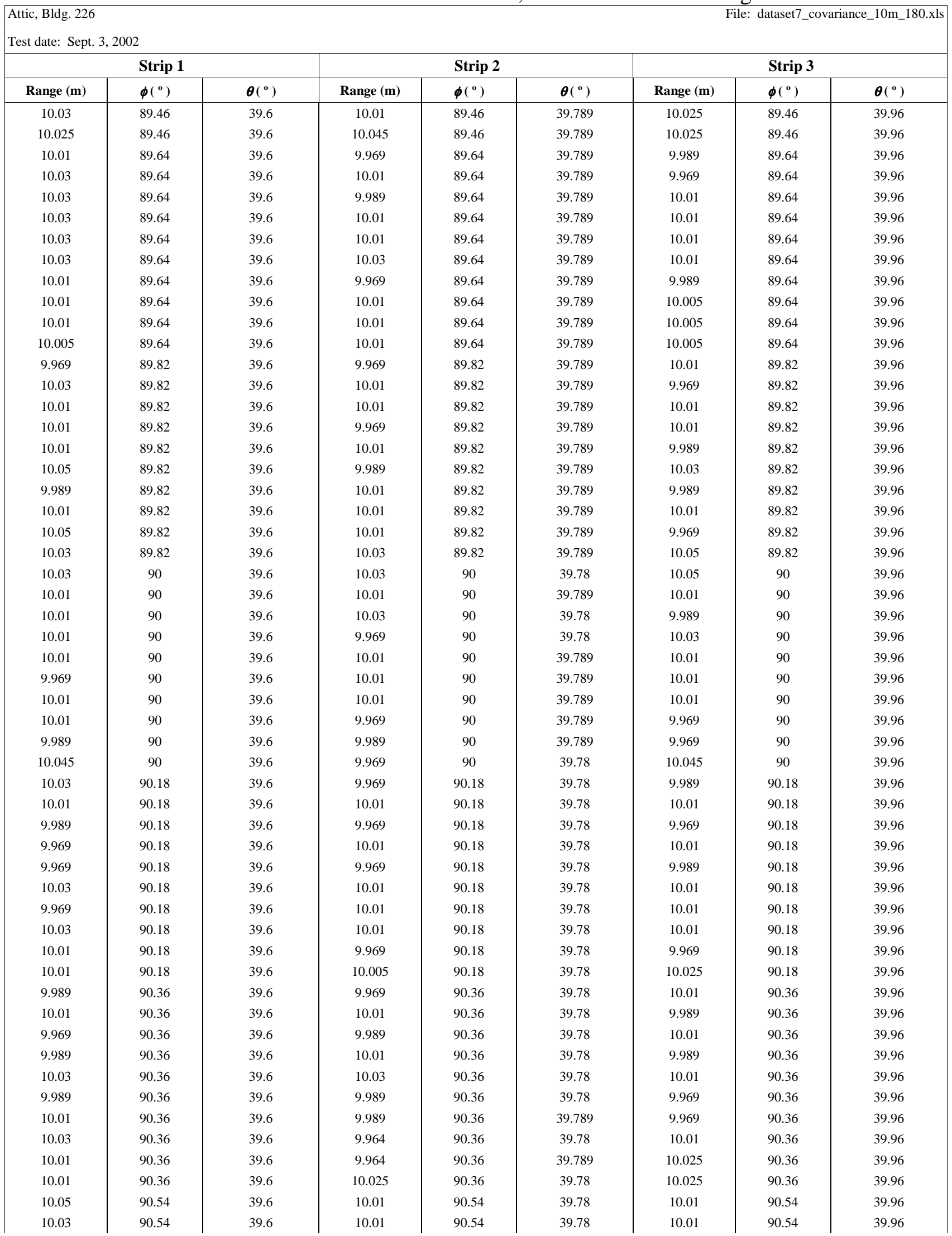


Table B.6c. Data Set 7: Covariance, $0.180^{\circ}$ Incremental Angle.

\begin{tabular}{|c|c|c|c|c|c|c|c|c|}
\hline \multirow{2}{*}{\multicolumn{3}{|c|}{\begin{tabular}{|l|} 
Attic, Bldg. 226 \\
Test date: Sept. 3, 2002 \\
Strip 1
\end{tabular}}} & & & & \multicolumn{3}{|c|}{ File: dataset7_covariance_10m_180.xls } \\
\hline & & & \multicolumn{3}{|c|}{ Strip 2} & \multicolumn{3}{|c|}{ Strip 3} \\
\hline Range (m) & $\phi\left({ }^{0}\right)$ & $\theta\left({ }^{\circ}\right)$ & Range (m) & $\phi\left({ }^{0}\right)$ & $\theta\left({ }^{\circ}\right)$ & Range (m) & $\phi\left({ }^{0}\right)$ & $\theta\left({ }^{\circ}\right)$ \\
\hline 10.01 & 90.54 & 39.6 & 10.01 & 90.54 & 39.78 & 9.989 & 90.54 & 39.96 \\
\hline 10.01 & 90.54 & 39.6 & 10.01 & 90.54 & 39.78 & 10.01 & 90.54 & 39.96 \\
\hline 9.969 & 90.54 & 39.6 & 10.03 & 90.54 & 39.78 & 10.01 & 90.54 & 39.96 \\
\hline 10.03 & 90.54 & 39.6 & 10.01 & 90.54 & 39.789 & 9.989 & 90.54 & 39.96 \\
\hline 9.969 & 90.54 & 39.6 & 9.969 & 90.54 & 39.789 & 9.989 & 90.54 & 39.96 \\
\hline 9.969 & 90.54 & 39.6 & 10.025 & 90.54 & 39.789 & 9.989 & 90.54 & 39.96 \\
\hline 9.989 & 90.54 & 39.6 & 10.005 & 90.54 & 39.789 & 10.01 & 90.54 & 39.96 \\
\hline 10.03 & 90.54 & 39.6 & 9.984 & 90.54 & 39.789 & 10.03 & 90.54 & 39.96 \\
\hline 9.969 & 90.72 & 39.6 & 10.03 & 90.72 & 39.78 & 9.969 & 90.72 & 39.96 \\
\hline 10.03 & 90.72 & 39.6 & 9.969 & 90.72 & 39.78 & 10.01 & 90.72 & 39.96 \\
\hline 10.03 & 90.72 & 39.6 & 10.01 & 90.72 & 39.78 & 9.969 & 90.72 & 39.96 \\
\hline 10.01 & 90.72 & 39.6 & 9.969 & 90.72 & 39.78 & 9.969 & 90.72 & 39.96 \\
\hline 9.989 & 90.72 & 39.6 & 10.01 & 90.72 & 39.78 & 10.01 & 90.72 & 39.96 \\
\hline 10.01 & 90.72 & 39.6 & 10.01 & 90.72 & 39.78 & 10.01 & 90.72 & 39.96 \\
\hline 9.989 & 90.72 & 39.6 & 9.969 & 90.72 & 39.78 & 10.01 & 90.72 & 39.96 \\
\hline 10.01 & 90.72 & 39.6 & 9.984 & 90.72 & 39.78 & 10.01 & 90.72 & 39.96 \\
\hline 10.05 & 90.72 & 39.6 & 10.03 & 90.72 & 39.78 & 10.01 & 90.72 & 39.96 \\
\hline 10.03 & 90.72 & 39.6 & 9.989 & 90.72 & 39.78 & 10.01 & 90.72 & 39.96 \\
\hline 9.969 & 90.9 & 39.6 & 10.01 & 90.9 & 39.78 & 9.989 & 90.9 & 39.96 \\
\hline 9.969 & 90.9 & 39.6 & 10.01 & 90.9 & 39.78 & 9.969 & 90.9 & 39.96 \\
\hline 9.989 & 90.9 & 39.6 & 10.03 & 90.9 & 39.78 & 10.01 & 90.9 & 39.96 \\
\hline 10.03 & 90.9 & 39.6 & 9.989 & 90.9 & 39.78 & 10.03 & 90.9 & 39.96 \\
\hline 10.01 & 90.9 & 39.6 & 10.01 & 90.9 & 39.78 & 9.969 & 90.9 & 39.96 \\
\hline 9.969 & 90.9 & 39.6 & 9.989 & 90.9 & 39.78 & 9.989 & 90.9 & 39.96 \\
\hline 10.03 & 90.9 & 39.6 & 10.03 & 90.9 & 39.78 & 10.01 & 90.9 & 39.96 \\
\hline 10.03 & 90.9 & 39.6 & 9.969 & 90.9 & 39.78 & 9.989 & 90.9 & 39.96 \\
\hline 10.03 & 90.9 & 39.6 & 9.989 & 90.9 & 39.78 & 10.03 & 90.9 & 39.96 \\
\hline 10.05 & 90.9 & 39.6 & 9.969 & 90.9 & 39.78 & 10.01 & 90.9 & 39.96 \\
\hline 10.03 & 91.08 & 39.6 & 10.05 & 91.08 & 39.78 & 10.01 & 91.08 & 39.96 \\
\hline 10.03 & 91.08 & 39.6 & 9.969 & 91.08 & 39.78 & 10.03 & 91.08 & 39.96 \\
\hline 9.989 & 91.08 & 39.6 & 9.989 & 91.08 & 39.78 & 10.01 & 91.08 & 39.96 \\
\hline 10.01 & 91.08 & 39.6 & 10.05 & 91.08 & 39.78 & 10.01 & 91.08 & 39.96 \\
\hline 10.05 & 91.08 & 39.6 & 9.989 & 91.08 & 39.78 & 9.989 & 91.08 & 39.96 \\
\hline 10.01 & 91.08 & 39.6 & 9.989 & 91.08 & 39.78 & 10.03 & 91.08 & 39.96 \\
\hline 10.05 & 91.08 & 39.6 & 10.01 & 91.08 & 39.78 & 10.05 & 91.08 & 39.96 \\
\hline 10.01 & 91.08 & 39.6 & 9.989 & 91.08 & 39.78 & 9.989 & 91.08 & 39.96 \\
\hline 9.989 & 91.08 & 39.6 & 10.01 & 91.08 & 39.78 & 10.03 & 91.08 & 39.96 \\
\hline 10.01 & 91.08 & 39.6 & 10.03 & 91.08 & 39.78 & 10.01 & 91.08 & 39.96 \\
\hline 10.01 & 91.26 & 39.6 & 10.01 & 91.26 & 39.78 & 9.969 & 91.26 & 39.96 \\
\hline 10.03 & 91.26 & 39.6 & 10.01 & 91.26 & 39.78 & 10.03 & 91.26 & 39.96 \\
\hline 10.01 & 91.26 & 39.6 & 9.969 & 91.26 & 39.78 & 10.03 & 91.26 & 39.96 \\
\hline 10.03 & 91.26 & 39.6 & 10.01 & 91.26 & 39.78 & 10.03 & 91.26 & 39.96 \\
\hline 9.969 & 91.26 & 39.6 & 9.989 & 91.26 & 39.78 & 9.989 & 91.26 & 39.96 \\
\hline 10.01 & 91.26 & 39.6 & 10.01 & 91.26 & 39.789 & 10.01 & 91.26 & 39.96 \\
\hline 10.03 & 91.26 & 39.6 & 10.03 & 91.26 & 39.789 & 9.989 & 91.26 & 39.96 \\
\hline 10.01 & 91.26 & 39.6 & 9.989 & 91.26 & 39.789 & 9.989 & 91.26 & 39.96 \\
\hline 10.01 & 91.26 & 39.6 & 10.01 & 91.26 & 39.789 & 9.969 & 91.26 & 39.96 \\
\hline 10.01 & 91.26 & 39.6 & 10.01 & 91.26 & 39.789 & 10.01 & 91.26 & 39.96 \\
\hline 10.01 & 91.44 & 39.6 & 10.03 & 91.44 & 39.789 & 10.01 & 91.44 & 39.96 \\
\hline 10.01 & 91.44 & 39.6 & 9.989 & 91.44 & 39.789 & 9.969 & 91.44 & 39.96 \\
\hline 10.03 & 91.44 & 39.6 & 10.03 & 91.44 & 39.789 & 10.01 & 91.44 & 39.96 \\
\hline 10.01 & 91.44 & 39.6 & 10.01 & 91.44 & 39.789 & 10.01 & 91.44 & 39.96 \\
\hline 10.05 & 91.44 & 39.6 & 9.969 & 91.44 & 39.789 & 10.01 & 91.44 & 39.96 \\
\hline 10.01 & 91.44 & 39.6 & 9.969 & 91.44 & 39.789 & 10.03 & 91.44 & 39.96 \\
\hline
\end{tabular}


Table B.6c. Data Set 7: Covariance, $0.180^{\circ}$ Incremental Angle.

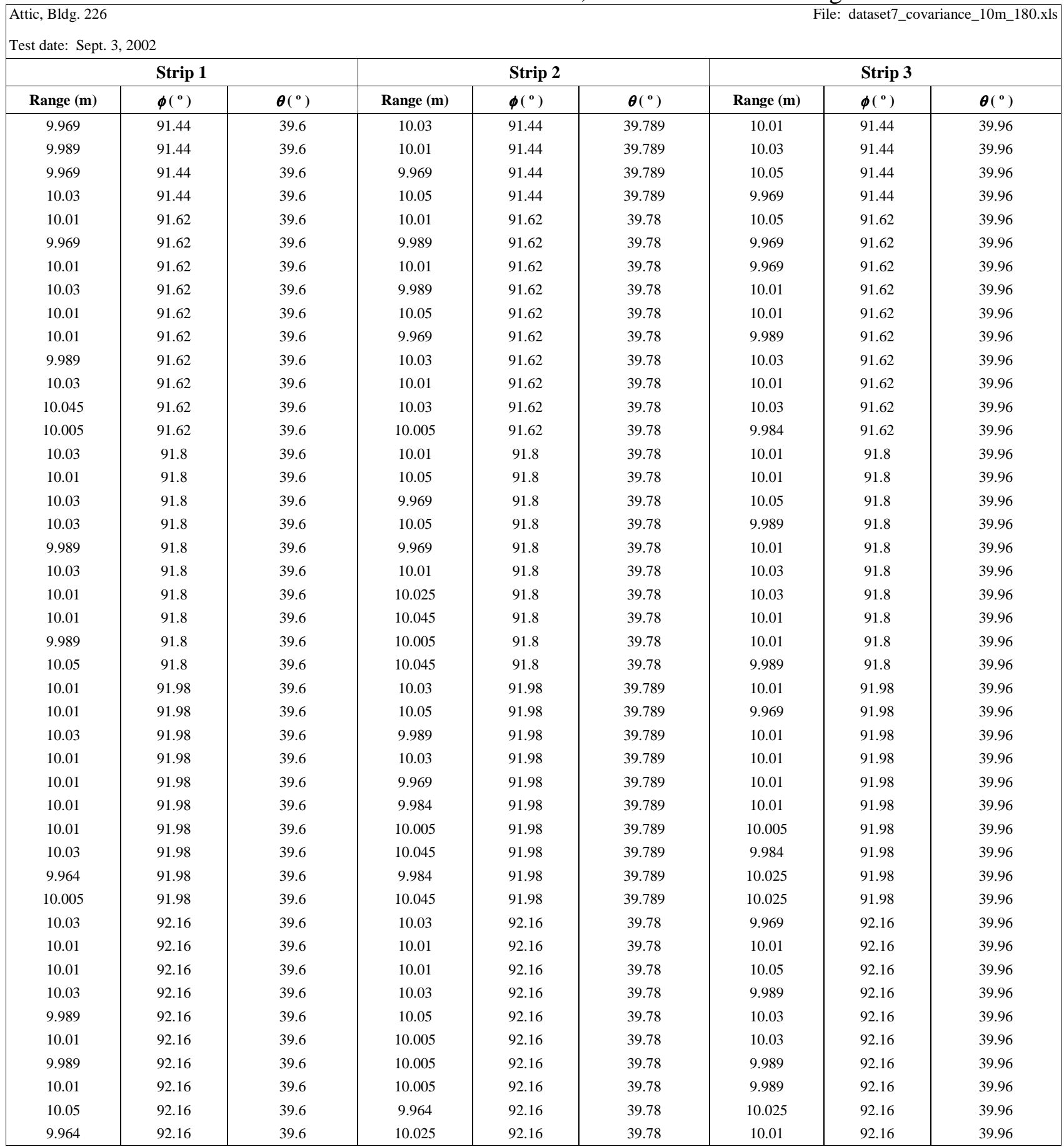


Table B.7. Data Set 8- Autocorrelation, 100 points

\begin{tabular}{|c|c|c|c|c|}
\hline & & & & File: 100 pts.txt \\
\hline Col.1 & Col. 2 & Col. 3 & Col. 4 & Col. 5 \\
\hline Distances & $\begin{array}{c}\text { Continued from } \\
\text { Col. } 1\end{array}$ & $\begin{array}{c}\text { Continued from } \\
\text { Col. } 2\end{array}$ & $\begin{array}{c}\text { Continued from } \\
\text { Col. } 3\end{array}$ & $\begin{array}{c}\text { Continued from } \\
\text { Col. } 4\end{array}$ \\
\hline (m) & $(\mathbf{m})$ & (m) & (m) & (m) \\
\hline 19.957 & 19.992 & 19.972 & 19.992 & 20.012 \\
\hline 19.997 & 19.992 & 19.972 & 19.992 & 19.988 \\
\hline 19.977 & 19.992 & 19.972 & 19.992 & 19.988 \\
\hline 20.017 & 20.012 & 20.012 & 19.992 & 19.988 \\
\hline 20.017 & 20.012 & 19.992 & 19.972 & 19.988 \\
\hline 19.997 & 19.992 & 20.012 & 19.992 & 20.012 \\
\hline 19.997 & 19.992 & 19.952 & 20.008 & 19.988 \\
\hline 19.977 & 19.972 & 20.012 & 19.972 & 19.968 \\
\hline 20.017 & 19.972 & 19.992 & 19.988 & 20.008 \\
\hline 19.997 & 19.992 & 19.972 & 19.968 & 19.988 \\
\hline 19.997 & 20.012 & 19.952 & 20.012 & 19.948 \\
\hline 19.997 & 19.992 & 20.012 & 20.012 & 19.998 \\
\hline 19.977 & 19.972 & 19.972 & 19.972 & 19.988 \\
\hline 19.997 & 19.992 & 19.992 & 19.992 & 20.008 \\
\hline 20.017 & 20.012 & 19.992 & 20.008 & 19.988 \\
\hline 19.997 & 19.992 & 20.012 & 19.988 & 19.968 \\
\hline 19.952 & 20.012 & 20.012 & 20.008 & 20.008 \\
\hline 19.972 & 19.972 & 19.972 & 19.992 & 19.948 \\
\hline 19.992 & 19.972 & 19.992 & 19.968 & 19.988 \\
\hline 19.992 & 20.012 & 20.008 & 20.008 & 19.988 \\
\hline
\end{tabular}

